\title{
Micromechanical Modeling of Dual Phase Steels
}

\author{
By \\ Fawzi Alabbasi \\ Department of Mechanical Engineering \\ McGill University \\ Montreal, Canada \\ September 2004
}

\begin{abstract}
A Thesis submitted to
McGill University

in partial fulfillment of the requirements for the degree of

Doctor of Philosophy
\end{abstract}

(c) Fawzi Alabbasi, 2004. 


$\begin{array}{ll}\begin{array}{l}\text { Library and } \\ \text { Archives Canada }\end{array} & \begin{array}{l}\text { Bibliothèque et } \\ \text { Archives Canada }\end{array} \\ \begin{array}{l}\text { Published Heritage } \\ \text { Branch }\end{array} & \begin{array}{l}\text { Direction du } \\ \text { Patrimoine de l'édition }\end{array} \\ \begin{array}{l}\text { 395 Wellington Street } \\ \text { Ottawa ON K1A 0N4 }\end{array} & \begin{array}{l}\text { 395, rue Wellington } \\ \text { Ottawa ON K1A ON4 } \\ \text { Canada }\end{array} \\ \end{array}$

Your file Votre référence

ISBN: 0-494-06267-3

Ourfile Notre référence

ISBN: 0-494-06267-3

NOTICE:

The author has granted a nonexclusive license allowing Library and Archives Canada to reproduce, publish, archive, preserve, conserve, communicate to the public by telecommunication or on the Internet, loan, distribute and sell theses worldwide, for commercial or noncommercial purposes, in microform, paper, electronic and/or any other formats.

The author retains copyright ownership and moral rights in this thesis. Neither the thesis nor substantial extracts from it may be printed or otherwise reproduced without the author's permission.
AVIS:

L'auteur a accordé une licence non exclusive permettant à la Bibliothèque et Archives Canada de reproduire, publier, archiver, sauvegarder, conserver, transmettre au public par télécommunication ou par l'Internet, prêter, distribuer et vendre des thèses partout dans le monde, à des fins commerciales ou autres, sur support microforme, papier, électronique et/ou autres formats.

L'auteur conserve la propriété du droit d'auteur et des droits moraux qui protège cette thèse. $\mathrm{Ni}$ la thèse ni des extraits substantiels de celle-ci ne doivent être imprimés ou autrement reproduits sans son autorisation.
In compliance with the Canadian

Privacy Act some supporting forms may have been removed from this thesis.

While these forms may be included in the document page count, their removal does not represent any loss of content from the thesis.
Conformément à la loi canadienne sur la protection de la vie privée, quelques formulaires secondaires ont été enlevés de cette thèse.

Bien que ces formulaires aient inclus dans la pagination, il n'y aura aucun contenu manquant. 


\section{DEDICATION}

\section{To my parents}




\section{Abstract}

Material characterization is a very important tool needed to describe and enhance material mechanical properties and to develop optimum material chemistries and microstructures. The usual approach of achieving the above using extensive experimental methods has been shown to be expensive and time consuming. This led to the development of the micro mechanical modeling, which can be used to predict the material behavior without the need for the extensive experimental investigation and is based on microstructural characteristics of the material.

In this work, a micro mechanical model is developed to predict the mechanical properties of dual phase steels consisting of martensite in a matrix of ferrite. This micro model is also used to elucidate the mechanics and mechanisms of deformation, which take place in such materials. DP-steels consisting of several volume fractions of martensite $\left(V_{m}\right)$ representing low, intermediate and high $V_{m}$ are developed and tested mechanically to obtain their mechanical properties. Metallographical examinations are carried out using image analysis to quantify microstructural material properties of each level of $V_{m}$ considered. As a validation of the current work, comparison between the model predictions, which include all the significant material behavior investigated in this work and the experimental results, is presented. The comparison demonstrates the ability of the model to capture the behavior of DP-steels up to the instability point.

The Gurson-Tvergaard model, which is the most widely known damage model to describe ductile failure, is coupled with the results of the micro mechanical model, presented in this work to form a complete material model of deformation and fracture of DP-steels. A procedure is developed to determine the parameters in the GursonTvergaard model utilizing the micromechanical model. The results are then implemented to simulate the deformation and failure of tensile bars of DP-steels with different $V_{m}$, which shows good agreement with the experimental results at failure. 


\section{Résumé}

La caractérisation des matériaux est un outil important pour décrire et améliorer les propriétés mécaniques des matériaux et développer des microstructures optimales. L'approche habituelle, nécessitant des méthodes expérimentales extensives, est très longue et dispendieuse. Ceci a donc donné lieu à la modélisation micro-mécanique qui peut être employée pour prédire le comportement des matériaux en se basant sur les caractéristiques micro-structurelles des matériaux et ce, sans le besoin d'études expérimentales.

Dans cet ouvrage, un modèle micro-mécanique est développé pour prédire les propriétés mécaniques d'aciers composés de martensite dans une matrice de ferrite. Ce modèle est aussi utilisé pour élucider les mécanismes de déformation dans ces matériaux. Des aciers à deux phases de plusieurs fractions volumétriques de martensite $\left(V_{m}\right)$, incluant des valeurs basses, intermédiaires et élevées de $V_{m}$, sont développés et testés pour obtenir leurs propriétés mécaniques. Des examens métallographiques ont été pratiqués avec de l'analyse d'imagerie pour quantifier les propriétés micro-structurelles à chaque valeur de $V_{m}$ investiguée. Pour valider cet ouvrage, une comparaison est établie entre des résultats expérimentaux et les prédictions du modèle, incluant tous les comportements significatifs des matériaux investigués. La comparaison démontre la capacité du modèle à reproduire les comportements des aciers à deux phases jusqu'au point d'instabilité.

Le modèle Gurson-Tvergaard, qui est le modèle de dommage le plus répandu pour la description des ruptures ductiles, est jumelé avec les résultats du modèle micromécanique pour offrir un modèle complet de la déformation et fracture d'aciers à deux phases. Une procédure basée sur le modèle micro-mécanique est aussi développée pour déterminer les paramètres du modèle Gurson-Tvergaard. Cette procédure est ensuite employée pour simuler la déformation et la fracture de barres en tension d'aciers à deux phases avec différents $V_{m}$. Ces résultats sont aussi en bon accord avec les résultats expérimentaux au point de rupture. 


\section{Acknowledgements}

The author wishes to express his gratitude to Professor James Nemes for his encouragement, guidance and supervision through the preparation of this work. Thanks also go to Professor Steve Yue in the metallurgy department for his advice and support in the metallurgical aspects in this research work and Dr. Bong-Yong Jeong who helped me in acquainting my self with the metallurgical equipments needed in this research work. The author would also want to thank Mr. Samuel Asamoah-Attiah for his help in familiarizing me with the MTS machine.

The author wishes to acknowledge the sponsorship and financial support of University of Bahrain. The author also wishes to acknowledge the support of the AUTO 21 Network of Centers of Excellence and the Natural Sciences and Engineering Research Council of Canada.

The author would like to especially express his extended and special thanks and appreciation to his wife for her patience, understanding and support. 


\section{TABLE OF CONTENTS}

Dedication................................................................

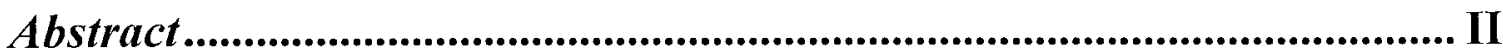

Résumé. ............................................................

Acknowledgements .................................................................................................. IV

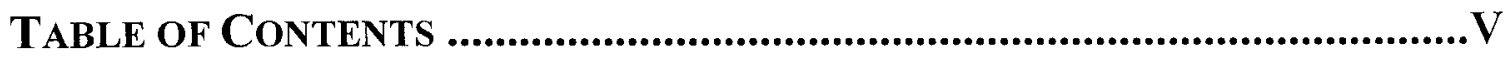

LIST OF FIGURES ..........................................................................................IX

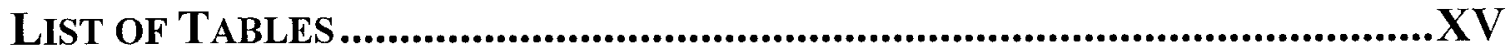

LIST OF

SYMBOLS..........................................................

Chapter 1 INTRODUCTION ........................................................... 1

1.1 Background .........................................................

1.2 The Structure of This Thesis ....................................... 4

Chapter 2 LITERATURE REVIEW. ................................6

$2.1 \quad$ Dual Phase Steels. .................................................

2.1.1 The Ferrite Phase .............................................. 10

2.1.2 The Martensite Phase ........................................12

2.1.2.1 Martensite Structure ..................................14

2.1.2.2 Martensite Hardness ....................................15

2.1.3 Mechanics of Deformation of DP-steels ........................16

2.1.4 Fracture Mechanism of DP-steels .............................20

2.2 Material Modeling Techniques. ..................................22

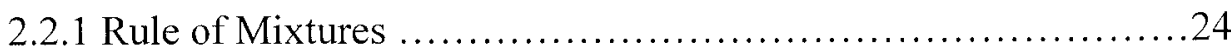

2.2.2 Micro Mechanical Modeling ..................................26

2.2.3 The Stochastic Moving-Window Micro

Mechanical Modeling. ..........................................29

2.2.4 Micro Mechanical Modeling of Cells. ...........................31 
2.3 Failure Mechanisms in Metals ...............................38

2.3.1 Void Nucleation. ............................................41

2.3.2 Void Growth and Coalescence. ...............................48

Chapter 3 ELASTO-PLASTIC MATERIAL MODELING...................56

3.1 Uniaxial Stress-Strain Loading................................57

3.1.1 Definition of Stress and Strain. ...............................57

3.1.2 Instability (Necking) of a Tensile Specimen. ..................59

3.1.3 Equivalent (Effective or Representative) Stress and Strain. .......60

3.1.4 Work Hardening. .......................................62

3.1 .5 Ductility. .............................................66

3.2 Plastic Behavior of Materials. ..................................67

3.2.1 Yield Criteria. ..........................................68

3.2.2 Post-Yield Behavior: Plastic Stress-Strain

Relations, Flow Rule. ....................................71

3.2.2.1 Classical Development of Flow Rules. ..................71

3.2.2.2 Generalized Plastic Stress-strain relations. ................73

3.2.2.3 Hardening Rules. .................................... 74

\section{Chapter 4 EXPERIMENTAL AND METALLURGICAL}

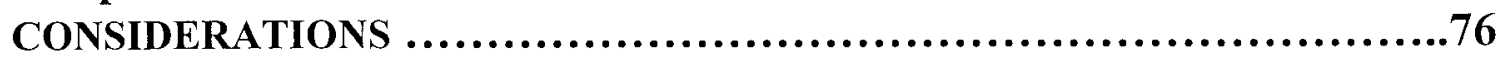

4.1 Metallography. ............................................ 77

4.1.1 Material Preparation for Microstructural Visualization ............78

4.1.2 Measurements and Image Analysis. ........................82

4.2 Materials and Experimental Procedures. ..........................86

4.2.1 Materials. ...............................................86

4.2.2 Mechanical Testing. .....................................88

4.2.3 Microstructure. ........................................ 92

Chapter 5 NUMERICAL MODELING OF DP-STEELS. .................96

5.1 Representative Volume Element (RVE). .......................97 
5.2 The Constitutive Behavior of Each Material Phase. .................100

5.3 Homogenization Method. ..................................104

5.4 Rule of Mixtures ............................................ 105

5.5 Finite Element Modeling Results. ............................. 108

5.6 Discussions and Conclusion. ................................. 119

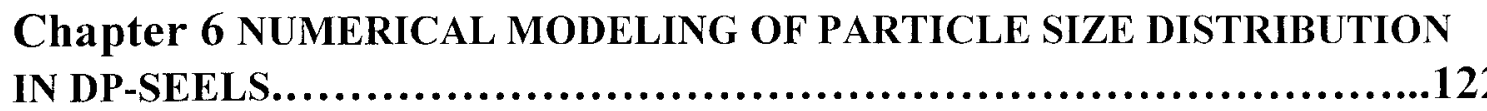

6.1 The Effect of Particle Size Distribution on Material Behavior. ....... 123

6.2 Finite Element Modeling. ..................................... 126

6.3 Discussions and Conclusion. ...................................135

Chapter 7 DUAL PHASE STEEL MATERIAL MODELING AND EXPERIMENTAL VALIDATION. ...............................138

7.1 Material Considerations. ........................................139

7.1.1: Martensite Softening. ...................................139

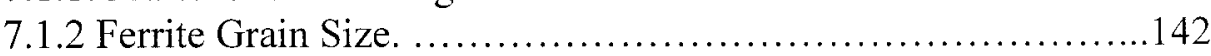

7.1.3 Particle Size Distribution. ................................. 142

7.2 Comparison Between experimental Results and Micro Mechanical

Model Predictions. ............................................... 145

7.2.1 Material Considerations. ....................................... 145

7.2.2 Comparison of Significant Aspects of

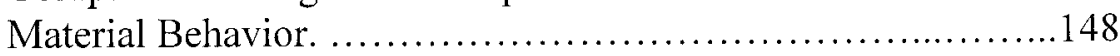

7.2.3 Discussion and Conclusion of the Comparison. ................152

7.3 Capturing the Effect of the Ferrite Grain Size on the Mechanical Properties of DP-Steels. .................................. 157

7.4 Constitutive Model Development. ............................... 161 
Chapter 8 MODELING THE DUCTILE FAILURE OF DP-STEELS BY VOID NUCLEATION, GROWTH AND COALESCENCE.

8.1 The Gurson-Tvergaard Model.................................... 64

8.2 Parameter Calibration Procedure.................................... 168

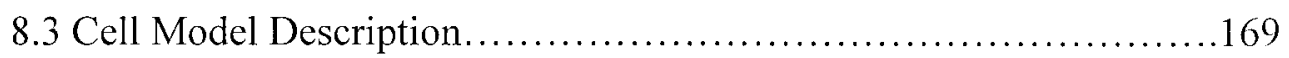

8.4 Parameter Determination....................................... 173

8.5 Simulation of a Tensile Bar Test.................................179

8.6 Conclusion.................................................. 184

Chapter 9 SUMMARY, CONCLUSIONS AND FUTURE

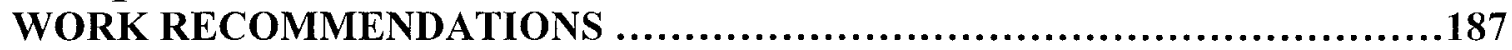

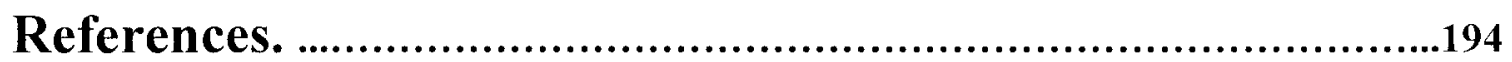




\section{LIST OF FigURES}

\section{Chapter 2 LITERATURE REVIEW}

Fig. 2.1: $\quad$ Standard $\mathrm{Fe}_{-} \mathrm{Fe}_{3} \mathrm{C}$ phase diagram (Askeland (1985)) . ....................

Fig. 2.2: The evolution of the microstructure of hypoeutectoid and hypereutectoid steels during cooling in relation to the phase diagram. .....................9

Fig. 2.3: The time temperature transformation diagram for a eutectoid steel. ..........10

Fig. 2.4: Effect of cooling rate on microstructure development. ......................13

Fig. 2.5: Martensite morphology as a function of carbon content. ...................14

Fig. 2.6: Lath martensite, needle-like shape and packet boundary in low carbon steel. ........................................................ 14

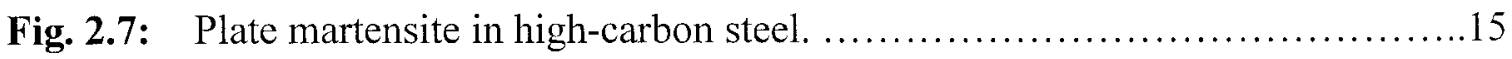

Fig. 2.8: Dependence of hardness on martensite volume fraction and carbon content. ............................................................ 15

Fig. 2.9: Two-dimensional plane strain material idealizations a) Simple square array (PS1) b) Staggered square array (PS2) c) Stacked hexagonal array (PS3)....32

Fig. 2.10: The stacked hexagonal array (SHA) model. (a) Three dimensional array of stacked hexagonal cylinders, each containing a spherical particle (b) The deformed and undeformed shape of the RVE under axial loading (c) The SHA axisymmetric RVE cell.

Fig. 2.11: Void nucleation models (a) cluster nucleation, (b) Continuous nucleation and (c) Statistical nucleation model. ...................................46

Fig. 2.12: Void nucleation, growth and coalescence in ductile metals. ...............51

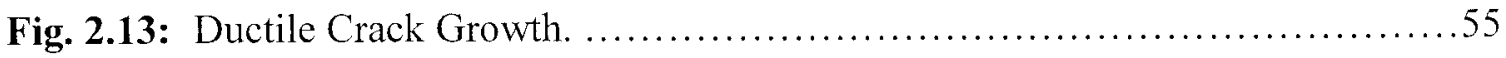




\section{Chapter 3 ELASTO-PLASTIC MATERIAL MODELING}

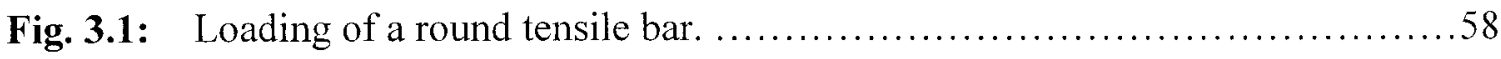

Fig. 3.2: Material subjected to combined state of stress. ........................61

Fig. 3.3: Plane strain case, of a long bar subjected to loading as shown. .............64

Fig. 3.4: (a) Von Mises flow stress surface in principal stress space (b) Synoptic plane. ..........................................................

Fig. 3.5: (a) Stress-strain curve in uniaxial tension and its effect on yield loading for (b) Isotropic hardening (c) Kinematic hardening and

(d) Combined hardening. .75

\section{Chapter 4 EXPERIMENTAL AND METALLURGICAL CONSIDERATIONS}

Fig. 4.1: Tensile specimen dimensions. .................................. 88

Fig. 4.2: Typical tensile specimen after fracture................................. 89

Fig. 4.3: Extensometer measurement of the engineering stress strain curve for $V_{m}$ of $14 \%, 19.7 \%$ and $34 \%$.

Fig. 4.4: MTS crosshead measurement of the engineering stress strain curve after correcting the final elongation to match the manually measured one for $V_{m}$ of $14 \%, 19.7 \%$ and $34 \%$.

Fig. 4.5: Representative engineering stress-strain curves for $V_{m}$ of $14 \%, 19.7 \%$ and $34 \%$.

Fig. 4.6: Single ferrite stress-strain curve, sample annealed to $1000^{\circ} \mathrm{C}$ for 21 hours....92

Fig 4.7: Micrographs of typical microstructures produced in this work a) $V_{m}=14 \%$, b) $V_{m}=19.7 \%$, c) $V_{m}=34 \%$ and d) Single ferrite phase with $6 \%$ pearlite. 


\section{Chapter 5 NUMERICAL MODELING OF DP-STEELS}

Fig. 5.1: Idealized behavior of the martensite and ferrite phases shown as true stress vs. true strain and engineering stress vs. engineering strain for uniaxial tensile stress conditions.

Fig. 5.2: Upper and lower bound predicted by ROM for several volume fractions $V_{m}$ a) $3.2 \%$ b) $13.6 \%$ c) $24.8 \%$ and d) $32 \%$.

Fig. 5.3: Dimensions of the micro mechanical model.

Fig. 5.4: Comparison of behavior predicted for the aggregate material using traction and displacement boundary conditions on side $\mathrm{S}_{4}$ for the PS1 model and $V_{\mathrm{m}}=11.8 \%$.

Fig. 5.5: Computed engineering stress vs. engineering strain under uniaxial tensile stress conditions for varying $V_{m}$ a) SHA model b) Plane strain (PS1) model c) Plane strain (PS2) model.

Fig. 5.6: Comparison of computed behavior using different models for low martensite volume fraction.

Fig. 5.7: Comparison of computed behavior using different models for high martensite volume fraction.

Fig. 5.8: Contours of equivalent plastic strain for nominal strains of 10\%, 20\%, and $30 \%$ and $V_{m} \approx 13 \%$ a) Plane strain (PS2) model b) Plane strain (PS1) model c) The SHA axisymmetric model.

Fig. 5.9: Contours of equivalent plastic strain for nominal strains of $10 \%, 20 \%$, and $30 \%$ and $V_{m} \approx 23 \%$ a) Plane strain (PS2) model b) Plane strain (PS1) model c) The SHA axisymmetric model.

Fig. 5.10: Contours of equivalent plastic strain for nominal strains of $10 \%, 20 \%$, and $30 \%$ and $V_{m} \approx 35 \%$ a) Plane strain (PS2) model b) Plane strain (PS1) model c) The SHA axisymmetric model.

Fig. 5.11: Predicted behavior using SHA axisymmetric model with $V_{m}=29.4 \%$ treating martensite as an elastic and an elastic-plastic solid. 


\section{Chapter 6 NUMERICAL MODELING OF PARTICLE SIZE DISTRIBUTION IN DP-STEELS}

Fig. 6.1: Typical microstructure of dual-phase steel showing the variation of the particle sizes.

Fig. 6.2: Axisymmetric two-particle model RVE idealization, showing the homogeneous dispersion of small and large particles.

Fig. 6.3: Dimensions of the micro mechanical models a) single particle model b) two particle model.

Fig. 6.4: The evolution of the equivalent plastic strain for the single particle model and the two particle model at particle size ratio, $\mathrm{R}=1$ and $V_{m}=13 \% \ldots \ldots 128$

Fig. 6.5: The predicted response of the two particle model at three different volume fractions of the harder phase a) $6.8 \%$ b) $17 \%$ c) $21.5 \%$ for particle size ratios of $\mathrm{R}=1, \mathrm{R}=3 / 4, \mathrm{R}=1 / 2, \mathrm{R}=1 / 3$ and $\mathrm{R}=1 / 16$.

Fig. 6.6: The predicted tensile strength of the aggregate at the particle size ratios, $\mathrm{R}=1, \mathrm{R}=3 / 4, \mathrm{R}=1 / 2, \mathrm{R}=1 / 3$ and $\mathrm{R}=1 / 16$ for $V_{m}=17 \%$

Fig. 6.7: Contours of equivalent plastic strain for nominal strain of $30 \%$,

a) $\mathrm{R}=1 / 4, V_{m}=9 \%$ b) $\mathrm{R}=1 / 4, V_{m}=13 \%$ c) $\mathrm{R}=1, V_{m}=17 \%$ and

b) d) $\mathrm{R}=1, V_{m}=22 \%$.

Fig. 6.8: Experimental results (Davies (1978)) of uniform strain vs. volume fraction of martensite compared to results using three modeling assumptions.

Fig. 6.9: Experimental results of strain hardening rate from Shen et. al (1986) compared to three modeling assumptions a) low $V_{\mathrm{m}}$ b) high $V_{\mathrm{m}}$. 134

\section{Chapter 7 DUAL PHASE STEEL MATERIAL MODELING AND EXPERIMENTAL VALIDATION}

Fig. 7.1: Carbon content for various $V_{m}$ extracted from a standard $\mathrm{Fe}-\mathrm{C}$ phase diagram using the lever rule. 140

Fig. 7.2: Predicted effect of carbon dilution of martensite phase on the behavior of DP-steels for $V_{\mathrm{m}}$ (a) $31.4 \%$, (b) $34.1 \%$ and (c) $55.4 \%$. 
Fig. 7.3: Contours of plastic strain with (left) and without (right)carbon dilution effect for $V_{\mathrm{m}}$ (a) $20.1 \%$, (b) $31.4 \%$, (c) $34.1 \%$ and (d) $55.4 \%$. 144

Fig. 7.4: Two martensite particles of ratio $\mathrm{R}=1 / 2$ dispersed in a matrix of ferrite. ...146

Fig. 7.5: Engineering and true stress-strain curves for single ferrite phase behavior fitted from the experimental results by two equations. ....................148

Fig. 7.6: True stress-strain diagrams for $V_{m}$ (a) $14 \%$, (b) $19.7 \%$ and (c) $34 \% \ldots \ldots .150$

Fig. 7.7: Engineering stress strain diagrams for $V_{m}$ (a) $14 \%$, (b) $19.7 \%$ and (c) $34 \%$.

Fig. 7.8: Strain hardening rate for a) $V_{\mathrm{m}}=14 \%$, b) $V_{\mathrm{m}}=19.7 \%$ and $V_{\mathrm{m}}=34 \%$. .154

Fig. 7.9-1: Contours of the plastic strain for a) $V_{\mathrm{m}}=14 \%$, b) $V_{\mathrm{m}}=19.7 \%$ at nominal strains of $10 \%, 20 \%$ and $30 \%$.

Fig. 7.9-2: Contours of the plastic strain for a) $V_{\mathrm{m}}=34 \%$ at nominal strains of $10 \%, 20 \%$ and $30 \%$. 156

Fig. 7.10: Comparison between the model predictions and the experimental results from Chang and Preban (1985) of the (a) Yield strength (b) Ultimate strength for $V_{\mathrm{m}}=11.4 \%$ at grain sizes depicted.

Fig. 7.11: Comparison between the model predictions and the experimental results from Chang and Preban (1985) of the (a) Yield strength (b) Ultimate strength for $V_{\mathrm{m}}=34 \%$ at grain sizes depicted 160

\section{Chapter 8 MODELING THE DUCTILE FAILURE OF DP-STEELS BY VOID NUCLEATION, GROWTH AND COALESCENCE}

Fig. 8.1: Effect of the parameter ' $q_{3}$ ' keeping the other parameters constant.

Fig. 8.2: Effect of ' $q_{1}$ ' keeping the other parameters constant.

Fig. 8.3: Effect of ' $q_{2}$ ' keeping the other parameters constant. 
Fig. 8.4: Schematic diagram outlining the procedure to obtain the parameters from the Gurson-Tvergaard model.

Fig. 8.5: Cell model with two springs and dimensions.

Fig. 8.6: Stress-strain response of the micro model for a) $V_{m}=14 \%$, b) $V_{m}=19.7 \%$ and c) $V_{m}=34 \%$.

Fig. 8.7 a: a) Contours of plastic strain for $V_{m}=14 \%$, void1 at $13.5 \%$ effective strain and details of void growth at b) $4 \%$ and c) $13.5 \%$ effective strain. ...176

Fig. 8.7 b: a) Contours of plastic strain for $V_{m}=14 \%$ for void 2 at $13.5 \%$ effective strain and details of void growth at b) $4 \%$ and c) $13.5 \%$ effective strain. ...177

Fig. 8.7 c: Contours of plastic strain for $V_{m}=19.7 \%$ for a) void1 at $14.2 \%$ and

b) void 2 at $15.6 \%$ effective strains and $V_{m}=34 \%$ for c) void1 at $9 \%$

d) void2 at $10 \%$ effective strains.

Fig. 8.8: Comparison between tensile bar model prediction and

representative experimental results for $V_{m}=14 \%, 19.7 \%$ and $34 \%$.

Fig. 8.9: Contours of plastic strain showing necking of the tensile bar model at fracture a) mesh design, b) $V_{m}=14 \%$, c) $V_{m}=19.7 \%$ and d) $V_{m}=34 \% \ldots .181$

Fig. 8.10: Predicted tensile bar responses from parameters determined in this work and the ones taken from Faleskog et al (1998) for Vm, a) 14\%, b) $19.7 \%$ and c) $34 \%$. 


\section{LIST OF TABLES}

Table 2.1: Calibrated values (optimal) of $\mathrm{q} 1$ and $\mathrm{q} 2$ of the Gurson

Tvergaard yield function.

Table 4.1: Steel chemical compositions in weight percent. ........................86

Table 4.2: Heat treatment details and volume fraction of martensite produced. .........87

Table 4.3: Mechanical properties of the DP-steels tested. .........................93

Table 7.1: Carbon content for each $V_{\mathrm{m}}$ tested experimentally...................... 146

Table 7.2: Predicted and experimental values of the ultimate strength and the uniform elongation for the range of $V_{m}$ considered in this work.

Table 7.3: Parameters used to describe the macroscopic material behavior.

162

Table 8.1: Variables determined from stress-strain response of voided micro model...174

Table 8.2: Parameters determined by solving the Gurson-Tvergaard yield function....174

Table 8.3: Calibration parameters obtained from results of Faleskog et al (1998)......183

Table 8.4: Comparison of the predicted results and the experimental findings,

ROA and elongation to fracture... .184 


\section{LIST OF SYMBOLS}

A Instantaneous area

$\mathrm{A}_{0} \quad$ Original area

$b \quad$ Magnitude of Burger's vector

BCC Body centered cubic

C $\%$ carbon content

d Grain diameter

$d_{w} \quad$ Specific plastic work

E Modulus of elasticity

e Nominal strain

$E_{i i} \quad$ Macroscopic strains in the $i i$ direction

$E_{e} \quad$ Macroscopic effective stress

$E_{i j} \quad$ Macroscopic average strain tensor

F $\quad$ Force

FCC Face centered cubic

$f \quad$ Yield function

$f_{v} \quad$ Void volume fraction

$f_{v}{ }^{*} \quad$ Effective void volume fraction

$f_{t} \quad$ Void total volume fraction

$f_{N} \quad$ Volume fraction of particle or inclusion

$f_{0} \quad$ Initial void volume fraction

$f_{\text {mcl. }} \quad$ Void volume fraction due to nucleation

$f_{g r .} \quad$ Void volume fraction due to growth

$f_{v, c} \quad$ Critical void volume fraction

$f_{v, F} \quad$ Void volume fraction at fracture 


\begin{tabular}{ll}
$f_{a}$ & Volume fraction of component "a" \\
$f_{b}$ & Volume fraction of component "b" \\
$\mathrm{G}$ & Shear modulus \\
$I_{i}$ & Stress invariants, $\mathrm{i}=1,2$ and 3 \\
$J_{i}$ & Deviatoric stress invariants, $\mathrm{i}=1,2$ and 3 \\
$k_{y}$ & Slope of the Hall-Petch equation related to yield strength \\
$k_{T}$ & Slope of the Hall-Petch equation related to tensile strength \\
$k_{v}$ & Bulk modulus \\
$L$ & Instantaneous tensile bar length \\
$L_{0}$ & Original tensile bar length \\
$\mathrm{M}$ & Magnification factor \\
$N_{\alpha}$ & Number of grains intercepted by the test line \\
$n$ & Hardening exponent (index) \\
$n_{f}$ & Hardening exponent for ferrite \\
$n_{m}$ & Hardening exponent for martensite \\
PS1 & Simple square array \\
PS2 & Staggered square array \\
PS3 & Axisymmetric stacked hexagonal array (also referred to by SHA) \\
$Q$ & Plastic potential \\
$q$ & Stress concentration factor \\
$q_{1}, q_{2}, q_{3}$ & Calibrating parameters in the Gurson-Tvergaard model \\
$\mathrm{R}$ & Particle size ratio \\
$r$ & Particle radius \\
$S_{i i}$ & Macroscopic stress in the $i$ i direction \\
$S_{e}$ & Macroscopic effective stress \\
$S_{h}$ & Macroscopic mean (hydrostatic) stress \\
$S_{N}$ & Standard deviation of void mean nucleation burst strain \\
$S_{i j}$ & Macroscopic average stress tensor \\
\hline
\end{tabular}


u

$\mathrm{u}_{i}$

UTS

V

$V_{i}$

$V_{m}$

Y
Thickness

Triaxiality

Tractions in the ith coordinate direction

Time temperature transformation

Displacement

Displacements imposed in the $i$ th coordinate direction

Ultimate tensile strength

Volume

Volume fraction of component $i$

Volume fraction of martensite

Yield stress

\section{Greek symbols}

$\begin{array}{ll}\varepsilon & \text { True strain } \\ \varepsilon_{e} & \text { Effective strain } \\ \varepsilon_{i j} & \text { Strain tensor } \\ \varepsilon_{i j}{ }^{\prime} & \text { Total strain tensor } \\ \varepsilon_{i j}{ }^{p} & \text { Plastic strain tensor } \\ \varepsilon_{i j}{ }^{e} & \text { Elastic strain tensor } \\ \varepsilon_{e}{ }^{p} & \text { Effective plastic strain } \\ \varepsilon_{N} & \text { Mean void nucleation burst strain } \\ \varepsilon_{d m} & \text { Induced microscopic austenite-martensite transformation strain } \\ \sigma & \text { True stress } \\ \sigma_{i j} & \text { Stress tensor } \\ \sigma_{i} & \text { Principal stresses, } \mathrm{i}=1,2 \text { and } 3 \\ \sigma_{i j}{ } & \text { Deviatoric stress tensor }\end{array}$




\begin{tabular}{|c|c|}
\hline$\sigma_{e}$ & Effective or equivalent stress \\
\hline$\sigma_{\mathrm{h}}$ & Mean or hydrostatic stress \\
\hline$\sigma_{L}$ & Local stress caused by dislocation pile-up \\
\hline$\sigma_{T}$ & Total maximum reference stress \\
\hline$\sigma_{E}$ & Elevated local stress \\
\hline$\sigma_{N}$ & Mean void nucleation stress \\
\hline$\sigma_{n(c)}$ & Thompson's critical stress \\
\hline$\sigma_{n}$ & Nominal stress \\
\hline$\sigma_{e f f}$ & Composite flow stress \\
\hline$\sigma_{a}$ & Flow stress due to component "a" \\
\hline$\sigma_{b}$ & Flow stress due to component "b" \\
\hline$\sigma_{y}$ & Yield stress \\
\hline$\sigma_{0}$ & Friction stress opposing movement of dislocations \\
\hline$\sigma_{c}$ & Critical stress \\
\hline $\bar{\sigma}$ & Hardening rule \\
\hline$\overline{\sigma_{m}}$ & Hardening rule for the martensite \\
\hline$\overline{\sigma_{f}}$ & Hardening rule for the ferrite \\
\hline$\mu$ & Shear modulus \\
\hline$\rho$ & Dislocation density \\
\hline$\Theta$ & Dilatancy \\
\hline$v$ & Poisson's ratio \\
\hline$\delta_{i j}$ & Kronecker delta \\
\hline$\gamma$ & Surface energy of particle \\
\hline$\gamma$ & Austenite \\
\hline$\alpha$ & Ferrite \\
\hline
\end{tabular}




\section{CHAPTER}

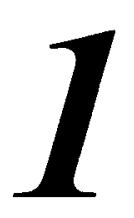

\section{INTRODUCTION}

\subsection{Background:}

In the last few decades, the manufacture of high quality products for industrial applications has become an important concern for a wide range of industries. Originating from the energy crisis of the 1970's, which added the factor of reducing fuel consumption, a new family of steels was developed, known as dual phase (DP) steels. Rigorous studies and research have been performed to develop products, which are economical, durable, and safe. 
It is well established now that low carbon multiphase (MP) steels, in general, developed in the past decades offer impressive mechanical properties, such as high work hardening rate and good ductility, which also have the advantage of reduced cost, superior formability, and excellent surface finish over other high strength low alloy (HSLA) steels. Experimental investigation into the effect of size, morphology and phase distribution of MP-steels has been comprehensively reported in the literature with focus on having two phases. The advantages of DP-steels were first reported by Rashid (1976). The pearlitic HSLA steels developed by micro alloying with different additives have shown significant increase in strength compared to the commercial plain carbon steels, but are inferior in terms of ductility and formability. Rashid and Cprek (1978) reported results of annealed vanadium alloyed steel, GM 980X, (a DP-steel) to display a promising decrease in the yield strength, higher ultimate strength, higher work hardening rate, and elimination of yield point elongation with considerable increase in ductility and formability. Tremendous efforts since the above report was released have been placed on exploring various aspects of DP-steels.

DP-steels offer attractive mechanical properties, especially for the automotive industry, such as high strength, continuous yielding, high work hardening rate and good ductility in addition to high crash resistance, reduced cost, good formability and excellent surface finish due to the elimination of the yield point elongation. The application of DP-steels in automotive components such as bumpers, wheels, wheel discs, pulleys, springs, etc. have shown a weight reduction of up to $30 \%$ with an increase in component life (Abdalla et al. (1999)). These steels are also reported to display high crashworthiness features due to the combined strength and ductility they display which is why they are used in the crash sensitive parts in the front and rear rails of automobiles.

DP-steels are produced by the intercritical heat treatment of low carbon steels. They possess a composite microstructure, which consists of any of martensite, bainite, pearlite or austenite in a matrix of a softer phase known as ferrite. Questions regarding optimum phase combinations persist and accurate predictive models are necessary to minimize the costly trial- and- error methods of development. A variety of homogenization techniques, such as rule of mixtures exist, but none of which could capture the real material behavior 
of the DP-steels in terms of the stress-strain trend and in terms of the mechanics of the deformation in the material composite due to the non-linearity and complexity of deformation process involved in such materials.

The unique mechanical behavior of DP-steels is attributable to their microstructure. The mechanism of failure of DP-steels reportedly occurs in a ductile manner by void nucleation, void growth and finally by void coalescence. The fracture mechanics approach, which is based on a well-founded mathematical background, fails to address this aspect of failure due to several reasons. The most important reason is that the basic philosophy in the conventional fracture mechanics which uses global fracture parameters such as the J-integral, works only in some limited cases, and often the assumption of the existence of a macroscopic flaw in the material does not correspond to the real material at hand and thus does not account for the characteristics of the material.

Consequently, modeling the mechanical behavior of the DP-steel materials has to be done based on the microstructural levels, which are many, but only the phase level of the material can be considered as an isotropic continuum. Micro mechanical models are used to understand the local mechanics and mechanisms governing the macroscopic deformation of the heterogeneous solid. They provide overall behavior from known properties of the individual constituents and their detailed interaction. In this work micro mechanical modeling of cells is used to characterize the DP-steels. Detailed investigations are presented and a unique model is developed which can particularly capture the behavior of materials which consist of two phases and which can also be extended to multiphase steels of more than two phases.

The micro mechanical model mentioned above could predict the deformation behavior of the material, which enabled it to predict the yield strength, UTS, the uniform strain and the strain-hardening rate. However, the micro mechanical model does not embody a fracture criterion, which enables it to predict a limiting strain to fracture. The ductile failure of DP-steels occurs by the process of void nucleation, void growth and finally void coalescence. For a void-containing material, the most widely known and used material 
damage model is the Gurson-Tvergaard model, which has been used comprehensively in the literature. The results of the micro mechanical model developed in this work are coupled with the Gurson-Tvergaard model to describe the deformation and fracture behavior of the DP-steels. The calibrating parameters for the Gurson - Tverggard model are determined uniquely by a procedure developed in this work

The main objective of the current research work is to develop a predictive model that can fully characterize DP-steel material behavior. The model above can then be used to understand the deformation and fracture behavior of DP-steels, which can lead to optimum design of these steels with enhanced properties. Micromechanical modeling is invoked in order to develop the predictive model mentioned above. Different idealizations and approaches are considered to see which one embodies the intrinsic ability to characterize DP-steels. Microstructures are developed and mechanical tests are performed in order to validate the predictive model above. In order to capture the fracture behavior of DP-steels, the Gurson-Tvergaard model is calibrated in a procedure developed in this work to obtain the proper parameters, which is also validated by comparing the predictions to the experimental results.

\subsection{The Structure of This Thesis:}

This Chapter presents the background and motivation for this research work and provides a brief description of the contents of each Chapter to follow.

Chapter 2: presents a literature review on the characterization and properties of the low carbon DP-steels as well as modeling techniques encountered in the literature to model different materials and the state reached related to this study.

Chapter 3: presents tensile test characterization of materials, measurement definitions that are used in this work, demonstration of some important aspects of tensile tests, which will be required later, and finally elaborates on the elastoplastic material modeling. 
Chapter 4: outlines experimental objectives, metallurgical considerations and measurements, experimental procedures and materials used in this research and experimental results.

Chapter 5: presents micro mechanical modeling developed in this study to capture the mechanical behavior of DP-steels. In this Chapter, the mechanics and mechanisms of deformation in the DP-steel investigation is presented both in terms of the stress-strain trend and in terms of the deformation mechanism in which the aggregate deforms.

Chapter 6: presents the effect of particle size distribution on the mechanical behavior of DP-steels.

Chapter 7: presents some essential material considerations required to capture the real material behavior of DP-steel. Comparison between the experimental results and the predicted model results are conducted to validate the numerical results experimentally.

Chapter 8: outlines the fracture mechanism in the DP-steel. It presents parameter selection and determination for modeling fracture of the DP-steel. A unique procedure to obtain the calibrating parameters in the Gurson-Tvergaard model is introduced. Comparison between experimental observations and modeling predictions for fracture is presented.

Chapter 9: a summary of this research work is presented, conclusions are drawn and future work recommendations are suggested. 


\section{CHAPTER}

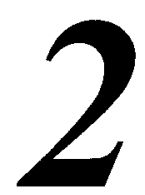

\section{LITERATURE REVIEW}

The starting point of this research work is materials that display very attractive mechanical features. Developing them would affect all fields of industry, especially the ones related to forming processes. The HSLA steels have been shown to display high strength compared to other steels, but they are inferior in terms of ductility and formability. Consequently, other types of HSLA steels have been developed with combined strength and ductility, which was first reported as mentioned earlier by Rashid (1976). The multiphase steel material behavior characterization and prediction is the most underlying aspect relevant to this study. Therefore, before going into material behavior prediction and modeling, it is essential to understand the material behavior aspects related to the material at hand which will be done next. 


\subsection{Dual Phase Steels:}

DP-steels consist of two phases coexisting in the material. They can consist of any of the secondary phases such as martensite, bainite, pearlite or austenite in a softer matrix called ferrite. DP-steels can be produced by intercritically annealing steel (heating between $A_{r l}$ and $A_{r 3}$ temperatures in the $\alpha+\gamma$ region) and then quenching to room or lower temperatures to produce the martensite or by annealing to some target temperatures using heating cycles which end up giving the desired second phase. Commercially, these steel structures are produced by either intercritically annealing HSLA steels or by heat treating the steel in the as rolled condition (Balliger and Gladman (1981)). Among all the second phases, martensite displays the most attractive features that influence the mechanical behavior of the steel aggregate, consisting of ferrite and the second phase. In addition, the heat treatment required to produce a DP-steel, which consists only of martensite and ferrite is easier and much cheaper compared to the other heat treatment processes to produce steels, which consist of the other phases. Due to the importance of the martensite and ferrite phases to this research work, the next section will be devoted to highlight the most important aspects of these phases pertinent to this study.

The term steel phase is referred to the form or structure of steel, which has the following characteristics (Askeland (1985)):

a) A phase has the same crystal structure or atomic arrangement throughout.

b) It has roughly the same composition and properties throughout.

c) There is a clear interface between the phase and any adjoining phases.

Many metals show polymorphism structure forms at different ambient temperatures. iron is one, which goes through two allotropic transformations during heating or cooling. Upon solidification, iron forms $\delta$-ferrite which is a BCC crystal structure form. Upon further cooling, the iron transforms to another phase called austenite or $\gamma$ which has an FCC crystal structure form. Finally, iron transforms back to a BCC crystal structure form at temperatures lower than around $912^{\circ} \mathrm{C}$, which is called $\alpha$, or ferrite. The ferrites and 
austenites form solid solutions of interstitial carbon atoms in iron. The solubility of the carbon atoms in the FCC crystal structures is much larger than that in the BCC crystal structure because the interstitial holes in the lattice of the FCC structures are larger than the holes in the lattice of the BCC crystal structures. This allows a greater number of carbon atoms to be accommodated in the FCC iron than the BCC iron, which has significant influence on the properties they display.

A eutectoid reaction is defined as a solid-state reaction in which one solid phase transforms to two other different solid phases:

$$
S_{1} \rightarrow S_{2}+S_{3}
$$

This reaction occurs in steel when the carbon content is about $0.77 \%$ on cooling below $727^{\circ} \mathrm{C}$. Hypoeutectoid steels contain less than $0.77 \% \mathrm{C}$ and hypereutectoid steels contain more than $0.77 \% \mathrm{C}$. The primary structure in the hypoeutectoid steels is ferrite and cementite $\left(\mathrm{Fe}_{3} \mathrm{C}\right)$ is the primary microconstituent in the hypereutectoid alloys as shown in Fig. 2.1 and Fig. 2.2 below.

DP-steels consisting of martensite in a matrix of ferrite can be produced by intercritically annealing the steel in the $\alpha+\gamma$ region (see Fig. 2.1) and then quenching rapidly to temperatures below the martensite start temperature (temperature at which martensite starts to develop- see Fig. 2.3). In order to avoid the formation of the other phases like pearlite and bainite, quenching has to be done fast enough as to avoid significant diffusion taking place. This is accomplished by cooling rapidly before reaching the nose of the TTT (time temperature transformation) diagram as depicted in Fig. 2.3. As martensite formation is a diffusionless process, it depends on the temperature only. Different volume fraction of martensite $\left(V_{m}\right)$ can be produced by annealing at higher temperatures in the intercritical region or by quenching to lower than room temperatures (Shehata and Crawley (1983)). 


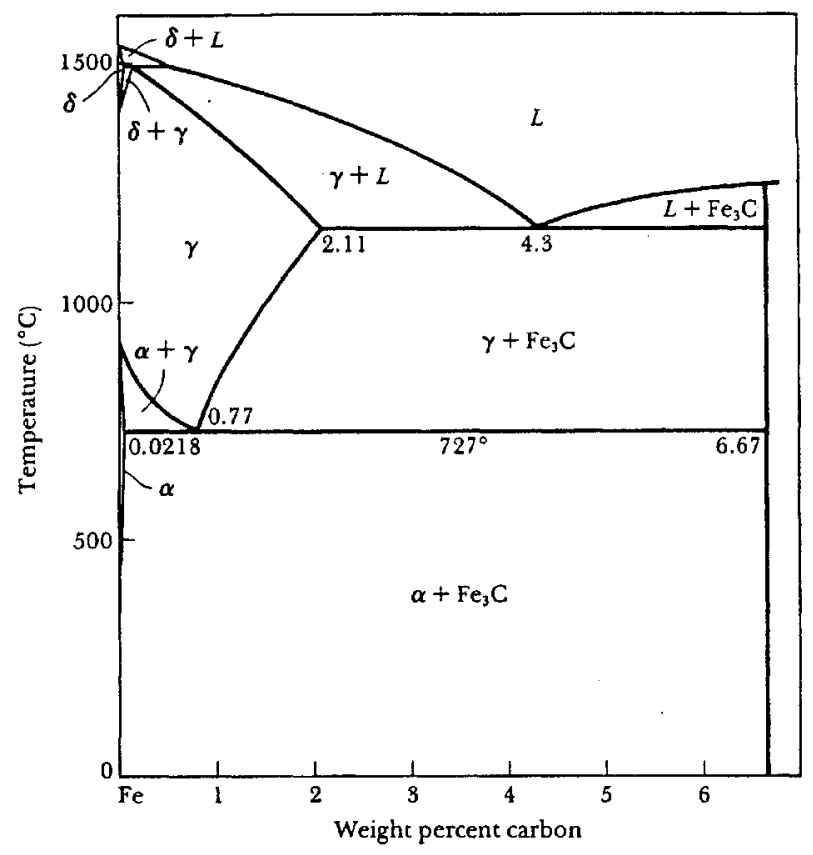

Fig. 2.1: Standard $\mathrm{Fe}_{-} \mathrm{Fe}_{3} \mathrm{C}$ phase diagram (Askeland (1985)).

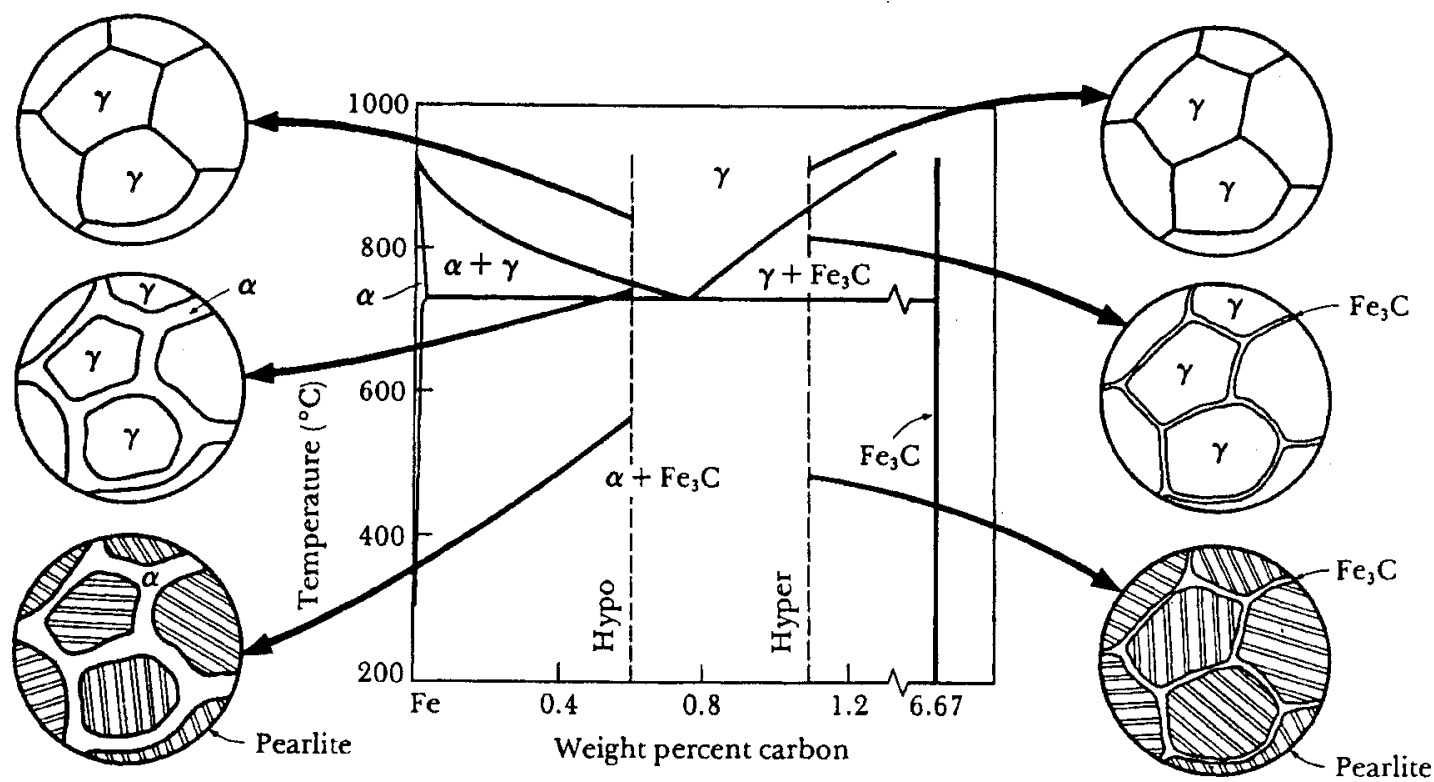

Fig. 2.2: The evolution of the microstructure of hypoeutectoid and hypereutectoid steels during cooling in relation to the phase diagram (Askeland (1985)). 


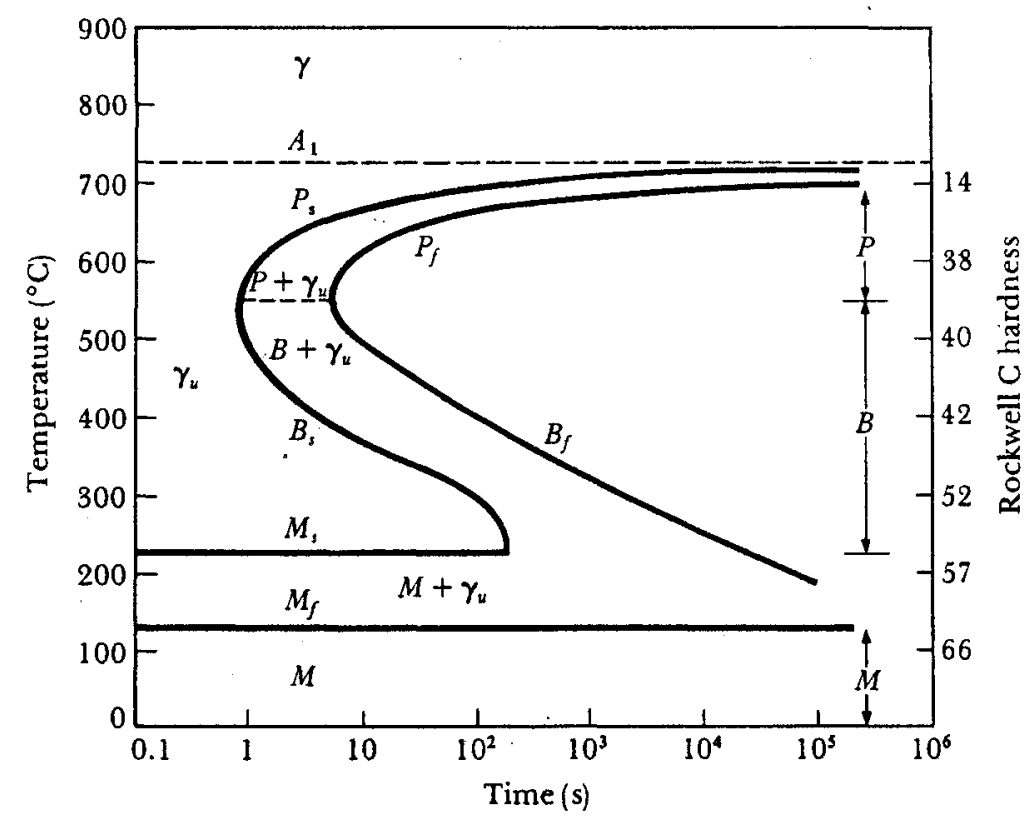

Fig. 2.3: The time temperature transformation diagram for a eutectoid steel (Askeland (1985)).

\subsubsection{The Ferrite Phase:}

Ferrite is the soft phase of steel, which can practically be called carbon-free iron. This phase has a body centered cubic (BCC) crystal structure which can contain only a few hundredths of a percent of carbon. The ferrite phase is the primary phase in the low carbon steels.

It is important to understand the strengthening mechanisms, which are operative in steels, the factors which control them and their effect on other properties such as toughness and ductility. Many excellent reviews of strengthening mechanisms have been made (Pickering and Gladman (1963), Gladman et al. (1972), Pickering (1978), Maynier et al. (1977), Petch (1953) and Hall (1951)) and only a brief general review will be given here. 
The major strengthening mechanisms can be attributed to:
a) Solid solution strengthening.
b) Precipitation or dispersion strengthening.
c) Dislocation or substructure strengthening.
d) Second phase strengthening
e) Grain size.

Empirical relationships which describe the effect of the above strengthening mechanisms to the yield strength have been developed which originate from Hall (1951) based on experimental observations and later by Petch (1953) based on theoretical and experimental means. They related the grain size to the yield strength by the equation referred to later as the Hall-Petch equation which, has the form:

$$
\sigma_{y}=\sigma_{0}+k_{y} d^{-1 / 2}
$$

where $d$ is the grain diameter, $\sigma_{y}$ is the yield stress, $\sigma_{0}$ is the friction stress opposing movement of dislocations in the grains and $k_{y}$ is a constant. A relationship of this form has been found to apply over grain sizes between 0.3 to $400 \mu \mathrm{m}$ in ferritic steels (Pickering (1978)). Pickering and Gladman (1963) have shown that the Hall-Petch equation, which described the increase of the yield strength with grain size may be extended to include both solid solution hardening terms as well as the terms involving dispersion strengthening. Afterwards, other strength contributions were added by others (Gladman et al. (1972), Pickering (1978), Maynier et al. (1977)), which took the form:

$$
\sigma_{y}=\sigma_{0}+\sigma_{S S S}+\sigma_{I S S}+\sigma_{P P T}+\sigma_{I S S}+\sigma_{S P H}+k_{y} d^{-1 / 2}
$$


Where $\sigma_{\text {SSS }}$ is the substitutional solid solution strengthening, $\sigma_{I S S}$ is the interstitial solid solution strengthening, $\sigma_{P P T}$ is the precipitation strengthening, $\sigma_{D S L}$ is the dislocation strengthening, and $\sigma_{S P H}$ is the second phase particle strengthening.

Morrison (1966) studied the effect of ferrite grain size on the strain hardening behavior of several low carbon steels. He reported that the Hall-Petch flow stress equation is found to be limited in validity but the lower yield stress equation is applicable over the full grain size range. He has shown that the trend of increasing the lower yield strength for steel of carbon content ranging from 0.05 to $0.2 \% \mathrm{wt} \mathrm{C}$ all fall within a small range and linearly increases with the reciprocal of the square root of the grain diameter.

Chang and Preban (1984) studied the effect of ferrite grain size for $\% \mathrm{C}$ contents of 0.034 , $0.07,0.17$ and 0.23 . They produced DP-steels with $V_{m}$ extending from $3.3 \%$ to $47 \%$. They varied the grain size of the ferrite from as small as $6.7 \mu \mathrm{m}$ to $59 \mu \mathrm{m}$ at a constant $V_{m}$ following the same heat treatment to avoid differences in properties of ferrite and martensite in the DP-steels. They reported that at roughly constant $V_{m}$ the dependence of the yield strength of a DP-steel on ferrite grain size obeys the Hall-Petch equation. They also noticed that with roughly constant properties in martensite and ferrite, the Hall-Petch slope, $k_{y}$, increases with increasing $V_{m}$. Finally, they have seen that the relation between the tensile strength of the DP-steel and the ferrite grain size also follow a Hall-Petch type equation with a different slope, $k_{T}$.

\subsubsection{The Martensite Phase:}

Martensite is the generic term for microstructures formed by diffusionless phase transformations. When the steel is annealed in the intercritical region it converts to austenite and ferrite, which make an FCC and BCC crystal structure form, respectively. At elevated temperatures, the absorption of the carbon atoms in the austenite is increased (FCC crystal structure) due to the rearrangement of the crystal structure, which allows the penetration of more carbon atoms into the austenite crystal structure. When the material 
is cooled slowly, normally the carbon atoms get released from the austenite by diffusion making up the ferrite and the pearlite (hypoeutectoid), but by rapid quenching not enough time is left for the carbon atoms to leave the austenite crystal structure, and thus they get trapped in the FCC crystal structure causing a volume expansion and distortion by transforming the steel to a BCT crystal structure, the martensite. In other words, martensite is the non-equilibrium phase that develops when austenite phase is quenched so rapidly that there is insufficient time for diffusion of the carbon atoms out of the austenite phase by which it would otherwise be transformed into ferrite and/or pearlite. The effect of cooling rate on micro structural development is shown in Fig. 2.4 below. The martensite phase transformation occurs by an atomic rearrangement across the transformation interface. That is, atoms in the parent austenite lattice are realigned into the lattice of the martensite phase. Since a diffusionless phase transformation produces martensite, the compositions of the martensite and parent austenite are the same (Cahn and Haasen (1996) and Porter and Easterling (1992)).

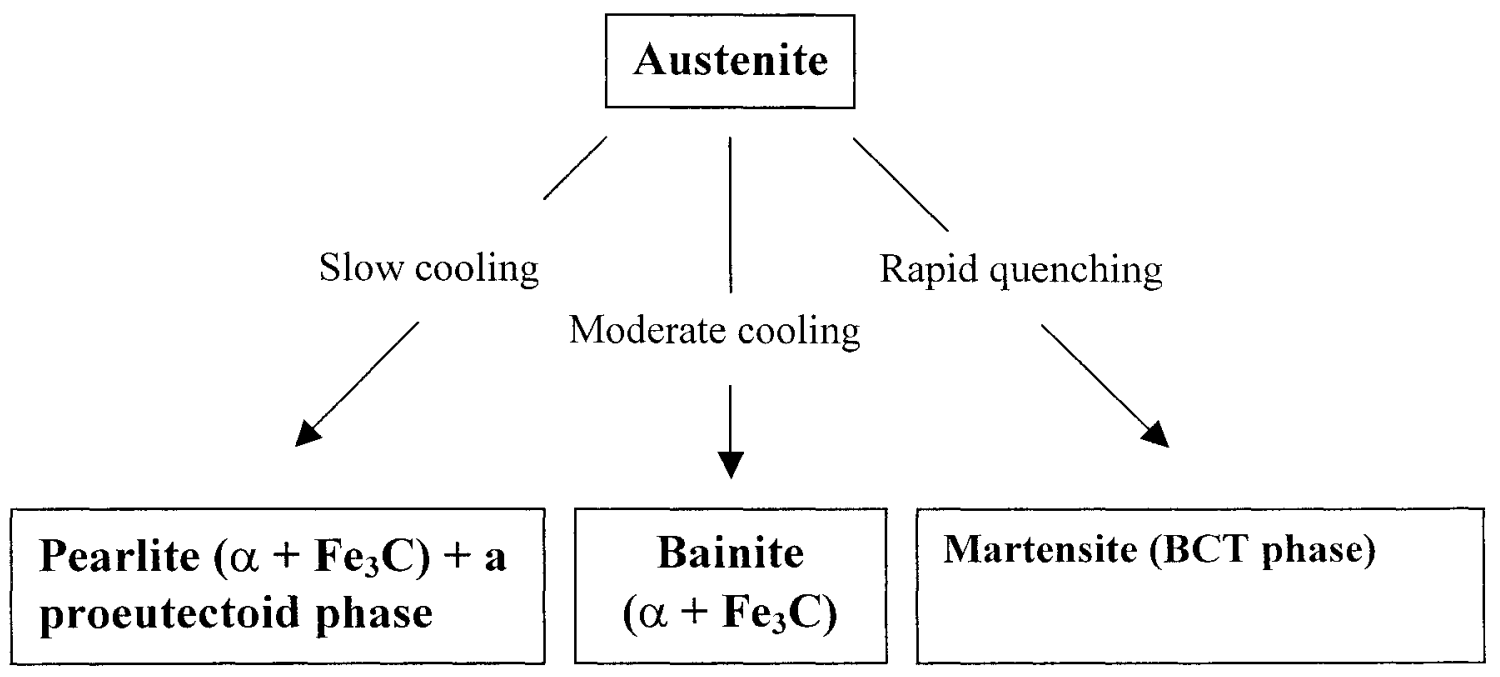

Fig. 2.4: Effect of cooling rate on microstructure development.

Compared to microstructures formed upon slow cooling, martensite has a fine structure and is much harder. Due to this, the martensitic transformation is an important phenomenon that can be used to significantly improve mechanical properties of the metal. 


\subsubsection{Martensite Structure:}

There are two basic forms of martensite structures, namely, lath martensite and plate martensite. The type that forms depends on the carbon content of the steel as depicted in Fig. 2.5 below (ASM (1985)).

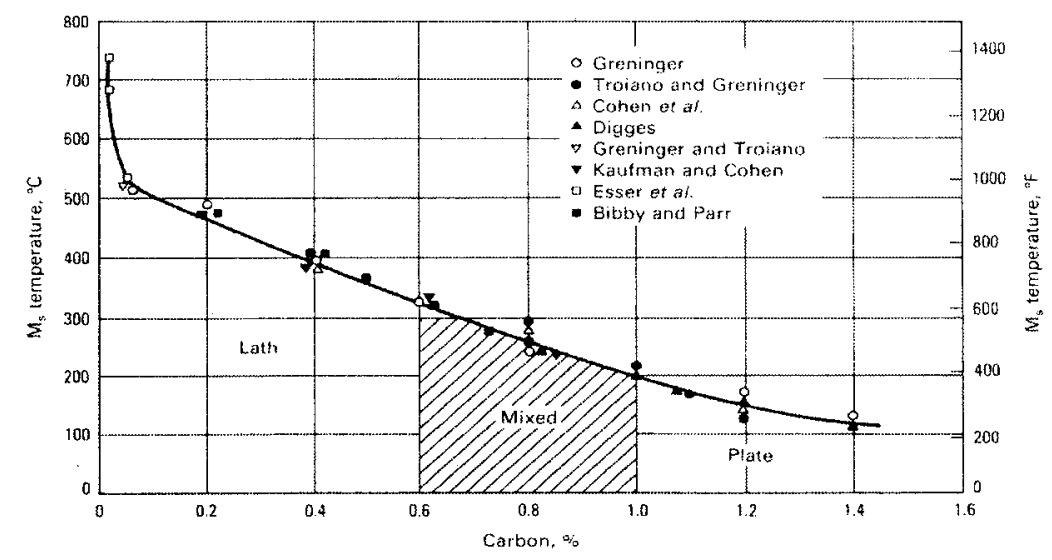

Fig. 2.5: Martensite morphology as a function of carbon content.

\section{a) Lath Martensite:}

Lath martensite structure is the most common martensitic structure. It is formed in low and medium carbon steels. The lath martensite structure is characterized by its needle-like shape and clustering into packets or colonies of similar crystal orientation (Honeycombe (1990), ASM (1985) and Cahn and Haasen (1996)) as depicted in Fig. 2.6 (Morris et al. (1999)).
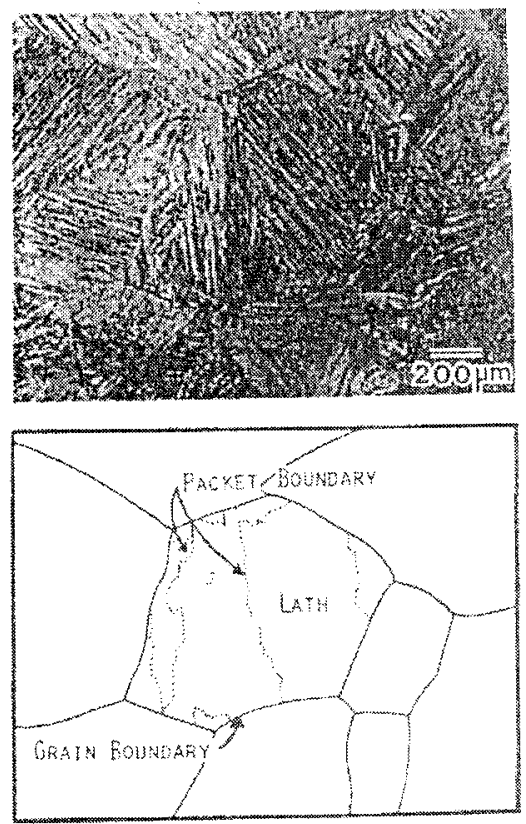

Fig. 2.6: Lath martensite, needle-like shape and packet boundary in low carbon steel. 


\section{b) Plate Martensite:}

Plate martensite forms in high carbon steels. It is also present in the medium carbon steels but in small amounts compared to the lath martensites. Plate martensite is characterized by plate-shape units. Unlike lath martensite, which forms into packets or colonies, plate martensite does not form in groups. Fig. 2.7 (ASM (1985)) shows a typical plate martensite structure in high-carbon steel.

\subsubsection{Martensite Hardness:}

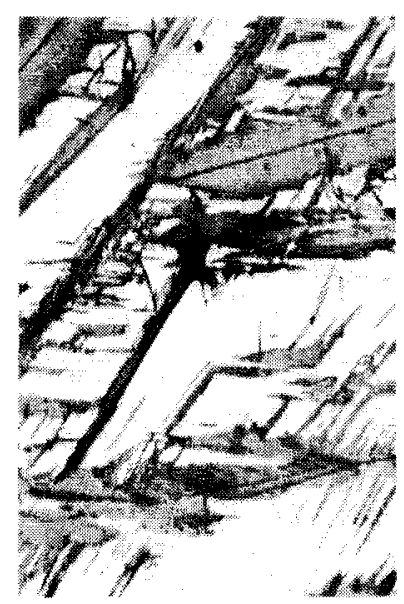

Fig. 2.7: Plate martensite in high-carbon steel

The relationship between steel carbon content, hardness, and martensite volume fraction is shown in Fig. 2.8. The steel hardness generally increases with increasing martensite volume fraction at constant carbon content. From Fig. 2.8, it can also be seen that specific hardness values can be achieved with lower $V_{m}$ by increasing the steel's carbon content. The hardness of martensite is reported to result from different factors depending on the carbon level of the steel. Interstitial solid solution strengthening dominates at high carbon content, while at low carbon levels; dislocation density is the major contribution to the hardness (Honeycombe (1990), Kelly and Nutting (1965) and Sinha (1989)).

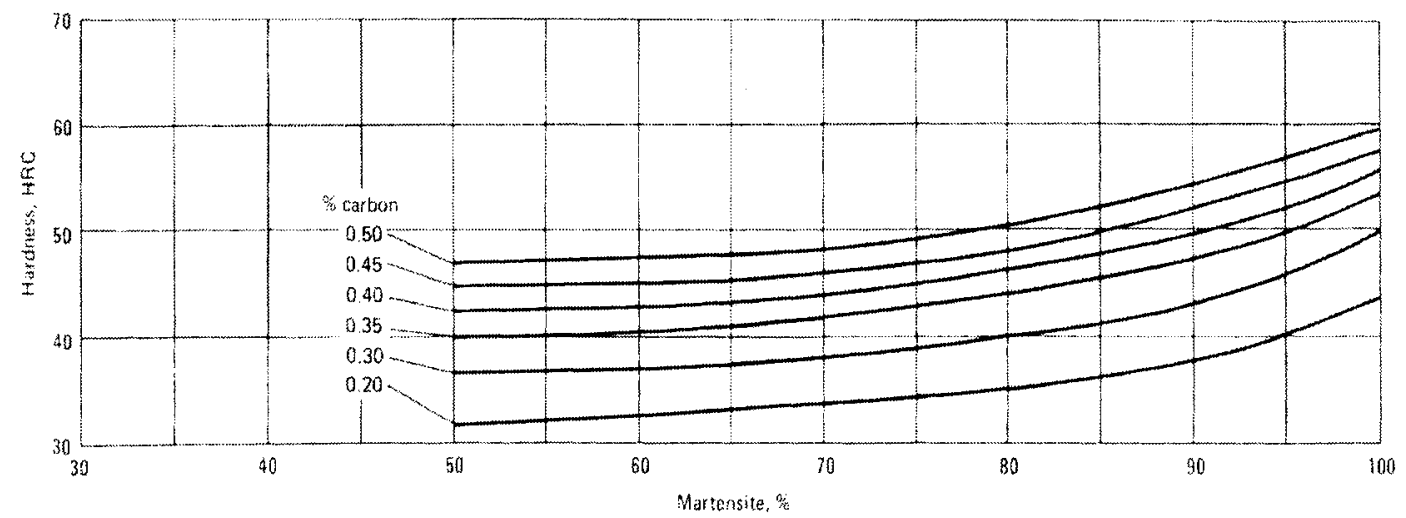

Fig. 2.8: Dependence of hardness on martensite volume fraction and carbon content (ASM (1990)). 


\subsubsection{Mechanics of Deformation of DP-Steels:}

The effect of $V_{m}$ has been investigated and reported widely in the literature. Increasing the volume fraction of the harder phase was found to increase the yield and ultimate strengths of the aggregate. Bag et al. (1999) reported that the increase in strength with $V_{m}$ only extends up to $V_{m} \sim 55 \%$ after which a reduction in strength is observed. The same was observed by Byun and $\operatorname{Kim}(1993)$ but at a different value of $V_{m}$. Shen, Lei and Liu (1986) have observed this as well without specifying the value of $V_{m}$ at which this takes place. Balliger and Gladman (1981) have reported a nonlinear increase of tensile strength with $V_{m}$ without commenting on the non-linearity. They attribute this to carbon dilution, which softens the martensite phase, thus dropping the overall strength of the aggregate. This can also be elucidated by considering the Joaul-Crussard analysis reported by Byun and Kim (1993), which will be shown shortly, where they show that the stages of strain hardening display three distinct regions for $V_{m}$ less than $30 \%$ and two stages of strain hardening for $V_{m}$ greater than $30 \%$. The stages of strain hardening have been reported by different authors to display three distinct regions:

1) Both component phases are elastic.

2) The softer phase is deformed plastically while the harder phase only elastically.

3) Both components deform plastically.

When the Joaul-Crussard analysis is employed, each region above corresponds to a distinct mechanism in the elastic-plastic deformation process (Bag et al. (1999) \& Byun and Kim (1993)). The Joaul-Crussard analysis is based on the following equation:

$$
\sigma=\sigma_{0}+k \varepsilon^{n}
$$

Where $\sigma_{0}$ is the reference true stress. Differentiating the above equation and taking the logarithm form we get: 


$$
\ln \left(\frac{d \sigma}{d \varepsilon}\right)=\ln k+\ln n+(n-1) \ln \varepsilon
$$

The equation above can be used to demonstrate the existence of different deformation stages in the DP-steels. This can be done by plotting $\ln \left(\frac{d \sigma}{d \varepsilon}\right)$ against $\ln \varepsilon$. Some authors (Jiang et al. (1993) and Tomita (1990)) have shown only two regions of strain hardening using the above analysis. Byun and Kim (1993) show that the strain hardening rate displays three distinct regions when $V_{m}<0.3$ and two regions when $V_{m}>0.3$. They relate this to the martensite phase becoming softer as its volume fraction increases. Other authors (Bag et al. (1999)) show that softening of the aggregate occurs at $V_{m} \sim 0.55$ and not $V_{m}=0.3$ and this explains why they get three distinct regions even though they used $V_{m}>0.3$. The difference is thought to be due to different alloying and morphology used in each investigation.

Shen, Lei and Liu (1986) have shown, using a scanning electron microscope equipped with a tensile straining stage, that the distribution of the strains between the ferrite and martensite phases, as well as among the different grains of each phase was observed to be inhomogeneous. They observed that the ferrite phase deformed immediately and at a much more rapid rate than the delayed deformation of the martensite. They add, that when the $\% \mathrm{C}$ is constant as $V_{m}$ increases, the difference in the strain in the two phases decreases in agreement with above observations mentioned in the last paragraph. Observations by Rashid (1978) also indicate that the martensite phase deforms after excessive straining of the ferrite matrix due to load being transferred to martensite islands through the martensite-ferrite interface. This is in contrast to other HSLA steels, where the ferrite phase deforms while the harder phase does not experience any significant deformation. The strain distribution between the phases in the DP-steels is believed to delay necking and strain to fracture as indicated by a higher strain rate index compared to the other HSLA steels. 
Davies (1978a, 1978b and 1978c) has also reported the increase of strength with $V_{m}$. He (Davies (1978c)) reported that the strength of the DP-steels is dependent on the ferrite grain size and the volume fraction of the martensite and is independent of the composition and strength of the martensite. This implies that martensite does not contribute to the deformation process much as he dealt with the aggregate as a matrix in which there is undeformable hard particles. In contrast to Davies (1978c), a number of studies have been made on the strength of martensite and suggested the dependency of martensite strength on the carbon content. Winchell and Cohen (1962) suggested a cube-root dependence, whereas Roberts and Owen (1965) and Clinton and Kelly (1968) have suggested square root dependence. Leslie (1981) and Leslie and Sober (1967) suggested a simple linear dependence for all ranges of martensite carbon content based on comprehensive experimental data. Speich and Warlimont (1968) reported square root carbon dependency for iron-carbon alloys containing 0.0004 to $0.18 \mathrm{wt} \% \mathrm{C}$ and showed that for higher carbon contents linear dependence reported by Leslie and Sober (1967) is quite close to their findings. Speich and Miller (1979) have used a linear carbon dependency in a study of mechanical properties of ferrite-martensite steels containing between 0.06 to $0.29 \%$ wt $\mathrm{C}$. They have shown that the tensile strength is increased when either martensite or its carbon content is increased. They have also shown that the yield strength $(0.2 \%$ offset $)$ also increases when either the amount of martensite or its carbon content is increased but the increase is less than the increase in the tensile strength. They attributed this to the residual stress patterns that influence initial flow properties. Byun and Kim (1993) have also shown carbon dilution effect in the martensite and used a linear relation reported by Leslie (1981) in a study of inhomogeneous deformation of ferrite-martensite DP-steels. They indicated that in a steel of high carbon martensite only ferrite phase plastically deforms so the stress strain partition was similar to that of particle hardened alloys. However, if the steel included a large amount of ductile martensite the stress and strain partition was similar to that of a duplex alloy containing two plastically deformable phases. Krauss (1977) presented a review on the athermal transformation kinetics, martensite start temperature as a function of composition, hardness, strength and structure of martensite. He has shown that the strength of martensite, in addition to being carbon dependent, is related to packet size of lath martensites, which is related according to the 
Hall-Petch relationship. Interestingly, a Hall-Petch slope that was steeper was found in the as quenched martensite than that observed in carbon free martensite i.e: the yield strength of as-quenched Fe-C martensite increased much more rapidly with decreasing packet size than did that of carbon-free martensite. The effect of martensite packet size has also been reported by Roberts (1970), Marder and Krauss (1970), Krauss and Marder (1971) and Swarr and Krauss (1976) to have an influence on the ultimate and yield strength of the martensite. They have shown that this effect is important in the as quenched martensite but minor in the quenched and tempered martensite. Kelly and Nutting (1965) have concluded after assessing the strengthening mechanisms in martensite that for plate and lath martensite, the effective hardening mechanism is due to carbon content by a square root relationship while the other mechanisms are negligible.

The effect of morphology of the second phase on different mechanical properties has also been investigated. Tomota (1987) has shown that the laminated and the continuous microstructures displayed higher strength than the isolated colonies microstructure. Bag et al. (1999) have shown that finely dispersed martensite in the form of needles in a matrix of ferrite are superior to banded and coarse grains of martensite. Tomita (1990) has shown that the islands of martensite microstructure gave a better combination of strength and ductility and produced a decreased yield ratio. Kang and Kwon (1987) have also shown that finely dispersed martensite displays higher ductility than a coarse plate like type when produced from lower intercritical temperatures. Sun and Pugh (2002) have shown that the strength and elongation not only depend on the $V_{m}$ but also the morphology of the second phase. Kim and Thomas (1981) have shown that the strength of the coarse martensite distribution is marginally larger compared to globular and finely distributed martensite while the former displays much loss of ductility compared to the latter ones. It could be concluded from the above that the morphology definitely affects the mechanical behavior of the MP-steels and that finer dispersed martensite provides a better combination of strength and ductility than the coarse plate like, banded and laminated morphologies. High strength is basically due to the resistance to dislocation movement because of the increase in the martensite-ferrite interface area and the better 
ductility due to the ferrite matrix being continuous which provides more ability to the softer ferrite matrix to deform plastically.

The austenite to martensite transformation expansion during quenching of steels from intercritical temperature results in plastic deformation of a region of the ferrite near each martensite particle. It was shown by Balliger and Gladman (1981) that the ferrite immediately surrounding martensite islands was observed to have high dislocation density. These result from the volume change associated with the austenite-to-martensite transformation. This localized ferrite plastic strain lowers the overall ductility of the ferrite and hence the DP-steel. The extent to which this happens has been studied theoretically by Bourell and Rizk (1983) by determining the extent of ferrite plastic zone, the effective overall increase in ferrite dislocation density and the associated strain, $\varepsilon_{d m}$.

\subsubsection{Fracture Mechanism of DP-steels:}

DP-steels have been reported by many authors to fracture in a ductile manner of void nucleation, void growth and void coalescence. Many investigators like Rashid (1977), Rashid (1978), Gladman (1997) and Balliger (1982) have observed that void formation arises from both martensite particle fracture and interface decohesion. Gladman (1997), Koo and Thomas (1977) and Balliger (1982) stressed that major voids form in the fracture of martensite particles. Sun and Pugh (2002) have observed that the formation of voids takes place by both mechanisms depending on the morphology of the martensite. Steinbrunner and Krauss (1988) observed three mechanisms of void formation, namely, interface decohesion, martensite fracture and uniquely identified martensite separation. Kang and Kwon (1987) studied the fracture behavior of intercritically treated structure in medium carbon steels and observed that the ferrite-martensite interface decohesion was the predominant mode of void nucleation and growth where martensite structure was the lath type. On the other hand, as the amount of the martensite increases, its shape change to the plate-like structure, which fails mostly by cleavage (low energy tears) rather than dimples observed in the former case. Kim and Thomas (1981) have reported that the initiation of the void in the DP-steels depends on the morphology of the second phase. 
They have shown in coarse martensite distribution, that the failure occurs by cleavage of ferrite grains, while for the globular and finely distributed martensite, the void initiation occurs at the ferrite-martensite interface and does not occur at the martensite particles as was reported by others. Others like Speich and Miller (1979), Korzekwa et al. (1980), Gerbase et al. (1979) and Szewczyk and Gurland (1982) have reported that void formation occurs due to martensite-ferrite interface decohesion. Speich and Miller (1979) observed that at low $V_{m}$, void formation occurs only due to interface decohesion and at high $V_{m}$ either mechanism forms voids as they have considered $V_{m}$ as high as $60 \%$. Szewczyk and Gurland (1982) have not observed any particle cracking for $V_{m}$ in the range $15-20 \%$.

Nam and Bae (1999) have shown that unlike martensite particles aligned nearly parallel to the drawing axis, which are thinned to fibrous shape, those aligned transverse to the drawing axis are severely bent and even fractured with increasing drawing strain. They stated that overwhelming reports find that the majority of voids which lead to fracture are formed at the ferrite martensite interface rather than the cracked martensite and eventually coalesce to cause failure during subsequent tensile loading or drawing. They showed that it is important that the martensite produced by heat treatment must be kept as deformable as possible to remain coherent with the ferrite matrix during cold deformation. This realignment of the martensite particles indicate that the martensite behaves anisotropically after excessive deformation.

\footnotetext{
Ahmed et al. (2000) have reported three modes of void nucleation, namely, martensite cracking, ferrite-martensite interface decohesion and decohesion at the interfaces with minimum plastic deformation, which has been uniquely identified by them. They reported that at low to intermediate $V_{m}$ the void formation was due to ferrite-martensite interface decohesion while the other two mechanisms also occurred at high $V_{m}$ (above $32 \%)$.
} 
From the above it can clearly be seen that void formation occurs mainly by both mechanisms, particle cracking and decohesion of martensite-ferrite interface, at high $V_{m}$ while at low and intermediate $V_{m}$ particle cracking is not observed.

\subsection{Material Modeling Techniques:}

It is fascinating to see the great variety of plastic behavior of materials. As a rule of thumb one can say there are as many models describing materials as much as there are materials. The approach used to describe the deformation and behavior of materials by mechanical and civil engineers has been somewhat different from that investigated by physicists and metallurgists. The former dealt with the phenomenological approach within the framework of continuum mechanics, boundary value problems and mathematical formulation while the latter dealt with the physical background of materials and especially devoted to the interrelation between the mechanisms of deformation and the evolution of microstructure. This is believed to result in the emergence of the micro and macro mechanical concepts in material modeling. The macro mechanical approach was mainly dealt with by mechanical and civil engineers and specifically promoted by people in the solid mechanics community while physicists and metallurgists used the micro mechanical approach. In the last two decades or more this gradually changed with the increasing demands for interdisciplinary researchers who can bridge between the two categories above. The need for this interdisciplinary category was realized by engineers and scientists due to the complexity of fracture of solids, which depends on a wide variety of factors including the macroscopic effects and the microscopic phenomenon which occur at locations where the fracture nucleates and grows.

The unique mechanical behavior of DP-steels is attributable to their microstructure. The mechanism of failure of DP-steels reportedly occurs in a ductile manner by void nucleation, void growth and finally by void coalescence. The fracture mechanics approach, which is based on a founded mathematical background, fails to address this aspect of failure due to several reasons. The J-integral, which is used as a fracture 
criterion, is derived based on elastic material response. Rigorous trials have been made in the past to extend the J-integral's applicability to the ductile fracture where extensive plastic deformation occurs. The deformation theory of plasticity has been invoked which is a non-linear elasticity theory where no unloading is permissible, in contrary to real material failure in ductile manner. In addition, the solution under the deformation theory of plasticity coincides with the incremental theory of plasticity when under proportional loading. This is argued by a number of researchers but this can actually be considered as an exception rather than general case. The basic philosophy in the conventional fracture mechanics which used global fracture parameters such as the J-integral, works only in some limited cases, especially since the assumption of the existence of a flaw from the beginning does not correspond to the real material in reality and thus does not account for the real characteristics of the material (Gdoutos (1993)).

The conventional modeling techniques, which are usually employed to explore component behavior using material behavior obtained experimentally, are termed macro mechanical approaches. In such approaches the heterogeneous material behavior is needed to perform such simulations. In doing so, the mechanical testing is required to be able to complete the simulation exercise. On the other hand, the techniques available in the micro mechanical modeling are based on micro structural aspects of the material under consideration and are generally based on homogenization procedures in different approaches which nullifies the need for testing the material mechanically which is the greatest advantage of micro mechanical modeling.

The type of modeling technique pertinent to this study are the ones which are based on micro structural aspects of the material, as in particular the DP-steels are reported to display unique attractive properties attributed to their microstructure. In the coming sections, micro mechanical modeling techniques and homogenization methods used to predict material behavior will be presented in brief but focus will be made on the micro mechanical modeling of cells, which is used in this research work. 


\subsubsection{Rule of Mixtures:}

Macroscopic properties of aggregates or composites consisting of two or more constituents can be obtained as the sum of the volume fraction of the components times their individual properties. This is known as the rule of mixtures, which is used to model composite materials, which comprise aggregates of two or more components or phases. Rule of mixtures are the most straightforward homogenization techniques known, of which a number of varieties exist. The simplest of these assume either uniform strain, known as the Voigt estimate, or uniform stress, which is known as the Reuss estimate. The two estimates have been shown by Hill (1963) to be the upper and lower bounds, respectively.

The rule of mixtures is specifically used in composite material behavior predictions in the longitudinal and transverse directions ${ }^{1}$. Some authors (e.g., Kim (2001)) have shown that the rule of mixtures could to a very good extent predict the deformation behavior of composites with homogeneously distributed soft and hard particles. It has also been specifically shown to be fairly accurate in the longitudinal direction of fiber composites. The material response in the longitudinal direction is like that of springs in parallel. The composite property is estimated as the sum of the responses of the composite components weighted by the component volume fractions as shown in the equation below (Whitney and McCullough (1990)):

$$
P=\sum_{i}^{\prime \prime} V_{i} P_{i}
$$

where $P$ is some property and $V$ is the volume fraction of the components. The material response in the transverse direction is like that of springs in series. The composite property is estimated as a reciprocal sum. The rule of mixtures is less accurate in the transverse direction as properties tend to be under-predicted. The equation takes the form:

\footnotetext{
${ }^{1}$ Longitudinal and transverse directions are the axial fiber direction and the direction perpendicular to the fiber axis.
} 


$$
\frac{1}{P}=\sum_{i}^{n} \frac{V_{i}}{P_{i}}
$$

For example, in terms of flow stress the two forms above, which are known as Voigt and Reuss estimates for a material consisting of two phases, can also be expressed in the following forms respectively:

$$
\begin{aligned}
& \sigma_{e f f}=f_{a} \sigma_{a}+f_{b} \sigma_{b} \\
& \frac{1}{\sigma_{e f f}}=\left(\frac{f_{a}}{\sigma_{a}}+\frac{f_{b}}{\sigma_{b}}\right)^{-1}
\end{aligned}
$$

where $f_{a}, f_{b}$ and $\sigma_{a}, \sigma_{b}$ are the volume fractions and the flow stresses of the constituents respectively.

More relevant to this work, rule of mixtures have been used by Bourell and Rizk (1983), in their modified rule of mixtures, which incorporate effects other than the mechanical behavior of each constituent such as the material changes due to thermal processes encountered in the process of material development (strain induced by austenite-tomartensite transformation), which induce stress and strain in the material that need to be realized as they might present significant influence in the material behavior. Other modified forms of rule of mixtures also exist which assume a ratio in the constant strain model (Voigt model) between the constituents, which is kept constant throughout the deformation process, and will be presented later.

There are also some other models like the composite spheres model, the self-consistent scheme, the generalized self-consistent scheme, the differential scheme, the Mori-Tanaka theory and Eshelby equivalent inclusion method. A good review of these approaches is given in Aboudi (1991) and Whitney and McCullough (1990). These approaches have 
been used for materials consisting of two elastic components mainly to predict the elastic modulus, and generally, would not be applicable for inelastic materials (Aboudi (1991)).

Aboudi (1991) also presents the micro mechanical method of cells, specifically for unidirectional fiber-reinforced materials and generalization of cells to short fibers and particulate composites is also presented. In the analysis an assumption is made, which is that geometry of the interface is insignificant and merely accounts for which phase is continuous and which phase is the inclusion and did not account for the interaction between neighboring particles, which is generally not true for aggregate materials and especially for the DP-steels. With this same assumption, the approach is extended to particulate and short fibers as the second phase or inclusion and to inelastic and viscoelastic material behavior. The elastoplastic material response is not presented and all the cases, which have been considered, are in the range of 1-2\% nominal strains. Discussions of above models are presented also in Whitney and McCullough (1990) with detailed descriptions of their shortcomings.

The same fundamentals were used to develop the micro mechanical modeling of cells and the stochastic moving-window approach presented below.

\subsubsection{Micro Mechanical Modeling:}

Micro mechanical analysis of MP-materials provides overall (aggregate) behavior from known properties of the individual constituents and their detailed interaction. On the other hand, in the macro mechanical approach, the heterogeneous structure behavior has to be known to predict the aggregate behavior using a computational model. There are many micro mechanical modeling approaches reported in the literature, all of which are based on the philosophy of capturing the material behavior based on its micro structural aspects. Micro mechanical modeling can be sought on many levels or scale of the microstructure. Each level is looked at by a discipline, which makes proper assumptions about the material. 
The unique mechanical properties of the MP-steels are attributable to their microstructure, which can be considered on several levels, all of which influence the final behavior of the product. At the finest, is the structure of individual atoms in space, which influence the electrical, magnetic, thermal and elastic behavior of the material. At the next level, is the arrangement of the atoms in space at which most metals retain a regular atomic arrangement or crystal structure. The crystal structure in each phase influences the mechanical properties of metals such as ductility and strength. Dislocations, imperfections happening in nature, exist and may be controlled to produce profound changes in properties. At the third level, is the grain structure; the crystal structure changes its orientation between grains and thus influences material properties. Finally, in most materials, more than one phase is present with each phase having its unique crystal structure and properties. The type, size, distribution, and amount of each phase e.g. volume or area fraction of each within the main body of the material can be controlled which provides an additional way to control the mechanical properties.

Modeling the microstructure on the first three levels would be numerically expensive, difficult to validate experimentally and non-cost effective if needed to produce and monitor on a large scale. It is reasonably easy and cheaper to model on the fourth level as each constituent of the material (each phase) can be considered a homogeneous isotropic part of the aggregate while from the atomic to the grain levels of structure, the properties are not realistically represented by an isotropic continuum. In addition, in modeling the material to capture its essential behavior requires a certain size of volume element, which embodies the essential features of the microstructure, and this is not feasible on the finer levels but, reasonably, possible on the phase structure level. Furthermore, controlling the microstructure on the finer levels requires addition of costly impurities in addition to the heat treatment processes required, unlike controlling the phase size, shape, volume fraction and distribution which can be achieved by only controlling the heat treatment processes that are well established in industry. Finally, considering the microstructure on this level will enhance unique properties already developed by alloying with additives and is considered as the ultimate enhancement, which can be achieved at low cost. 
The procedure by which micro mechanical modeling is explored is summarized as follows:

1) Selecting an appropriate scale at which the phenomenon of interest can be best described.

2) Observing the essential microstructures, which influence the modeling outcome significantly.

3) Employing the constitutive relations for the micro constituents considered.

4) Employing the behavior of the interface between the micro constituents, when ever applicable.

The selection of the scale of microstructure to be modeled is thought to be the most important aspect which influences the modeling procedure and output and care has to be given to this matter as it is the starting point of the modeling upon which all the results will be clinging. The consideration of the scales of the microstructure apply to all micro mechanical approaches without distinction. Some authors prefer to divide the range from the structure of individual atoms to the phase level into two categories, namely, micro and meso scales while the next level as macro scale. In this work the two finer levels will be referred to as micro scale levels.

There are two approaches reported in the literature which can be used to model the material properties based on the phase level, namely the micro mechanical modeling of cells and the stochastic moving-window modeling technique ${ }^{2}$. The micro mechanical modeling of cells is preferred and used in this work and thus only a brief description of the stochastic moving-window approach will be given here.

\footnotetext{
${ }^{2}$ Authors refer to this method also by micro mechanical modeling of cells but in this work distinction is made as the most visible nature of this method is using moving windows.
} 


\subsubsection{The Stochastic Moving-Window Micro Mechanical Modeling:}

The stochastic moving-window micro mechanical modeling was developed a little more than one decade back. Many researchers have used this approach (e.g., Aboudi (1991), Aboudi (1989), Aboudi (2000), Ostoja-Starzewski (1993), Ostoja-Starzewski (1994), Ostoja-Starzewski (2000), Ostoja-Starzewski (1999), Baxter and Graham (2000), Graham and Baxter (2000), Jeulin and Ostoja-Starzewski (2001), Sluis O. V D., et al. (2000) and Jiang et al. (2001)). The stochastic moving-window micro mechanical modeling is based on the assumption of the existence of a representative volume element (RVE) much larger than the micro scale which corresponds, for example, to the characteristic size of a fiber in a composite, a crystal in a metal or ceramic or a crack in a continuum and much smaller than the macroscopic characteristic length (Ostoja-Starzewski (1999)) which can result in a convergence of the stiffness and compliance of the material. This approach has emerged as a useful technique for characterizing microstructure of random composite materials. The major advantage of this method is that there is no beforehand assumption needed with regard to shape, size and distribution of the inclusions as it is based on the usage of digitized images of the microstructure. A certain size of this image is to be used that would represent the real material behavior; an investigation from which the name "moving-window" is originated as to select the proper size, that characterizes the real material behavior. The major difficulties in applying this method are summarized in the following:

1) Image resolution: The appropriate image resolution of the microstructure must be determined. If the image resolution is too fine, then it may become computationally forbidding and if the resolution is too coarse then there is too much significant microstructural information lost.

2) The window size: As mentioned earlier an optimum window size, which causes no change in the model response by any further increase on its size would be the target size sought. This is done randomly in one image but still in the other places 
of the microstructure the exact same microstructure evolution is not expected, which means one has to extend to other images also.

3) The scale response by the window method is non-unique as it depends on the type of loading imposed on its boundaries and might present anisotropic response, which in certain cases is not the real presentation of the material and contradicts Hill's (1963) definition of a well developed RVE.

4) The approach is based on the plain image of the material, and not a 3D representation, misinterpreting the material reality. The most important feature of the RVE prevails in that it should embody the essential features of the microstructure, for which this approach fails to address. It has been shown by Baxter and Graham (2000) in an investigation of the development of micromechanics based material property fields for random composites that the $3 \mathrm{D}$ analysis of the moving window gives significantly different results than the $2 \mathrm{D}$ version. They used commercial computer aided tomography and x-ray scanning to get $3 \mathrm{D}$ images and applied the window-moving approach to small portions of the image as they reported that modeling the $3 \mathrm{D}$ images would be prohibitive computationally. They used the stereological concept of dividing the image into small parts and dealing with each separately and use the overlap between the sizes chosen to represent the interaction between different locations of the microstructure. This method clearly requires many facilities and expertise to perform the investigations, which are not available for the present research.

5) The noise problem has to be resolved, as there would be very small particles in the material to be decided upon whether to model or not.

6) Although meshing techniques are growing fast in finite element packages, it still is difficult making meshes to resemble the images. 
7) The use of plane strain elements have been investigated in this work and have been seen not to represent the real material behavior from different aspects of characterization of DP-steels. Details about this investigation will be provided later in the results Chapter.

Due to the difficulties and problems mentioned above concerning the moving-window approach and due to some preliminary investigation on the micro mechanical modeling of cells, which showed its appropriateness for the DP-steels, it was decided to utilize this approach to perform this research work. Detailed description of the micro mechanical modeling of cells will be presented in the next section.

\subsubsection{Micro Mechanical Modeling of Cells:}

The micro mechanical modeling of cells as referred to in this work is normally used to understand the local mechanics and mechanisms governing the macroscopic deformation of heterogeneous solids. There are three basic features to a micro mechanical model for a generic multiphase composite:

a) Geometric definition of a representative volume element (RVE), which embodies the essential features of the microstructure.

b) The constitutive description of the mechanical behavior of each phase and the interface boundaries, whenever applicable.

c) A homogenization strategy (procedure) for macroscopic mechanical behavior of the aggregate based on the response of the RVE (Socrate and Boyce (2000)). 


\section{a) Representative Volume Element (RVE):}

A prominent feature of the micro mechanical modeling of cells is the transition from a medium with a periodic microstructure to an equivalent homogeneous continuum, which effectively represents the composite material. In a two-phase microstructure, a spatially periodic RVE is assumed to deform in a repetitive way and each RVE resembles its neighboring cells in all aspects. This is indeed a simplifying assumption, but has proved to be satisfactory and is widely accepted. The extent to which the RVE captures the behavior of the microstructure depends in a way on how accurately the RVE captures the morphological features of the actual microstructure (Socrate and Boyce (2000)).

Modeling the microstructure based on plane strain or axisymmetric cell models reduces the complexity of $3 \mathrm{D}$ modeling and minimizes the computational cost. Different idealizations based on plane strain or axisymmetric models are reported in the literature, all of which proved to capture the essential real material behavior to some degree. Huang and Kinloch (1992) concluded after comparing results of 3D models with previous work, that axisymmetric models could be used without significant loss in accuracy. The simplest plane strain idealization that is used to represent the periodic array of a twophase material is based on a simple square array (PS1) with axes of the array parallel to the loading direction, as shown in Fig. 2.9a.

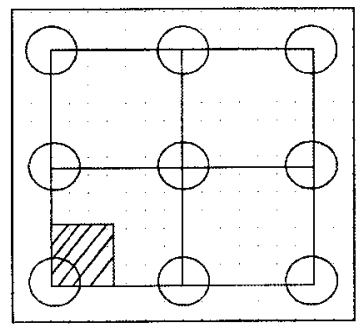

(a)

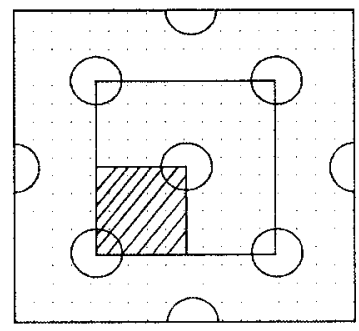

(b)

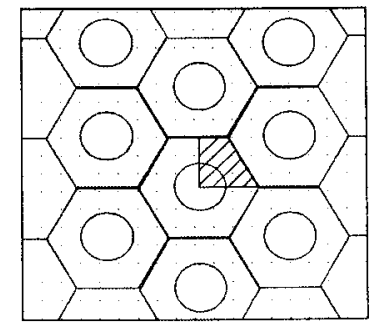

(c)

Fig. 2.9: Two-dimensional plane strain material idealizations a) Simple square array (PS1) b) Staggered square array (PS2) c) Stacked hexagonal array (PS3). 
As an alternative, the staggered square array (PS2), shown in Fig. 2.9 b, eliminates the inhomogeneous nature of the simple square array due to the periodic alignment of rows by the shift of the particles in every other row horizontally. The hexagonal array (PS3), shown in Fig. 2.9 c, has also been used by some researchers, and shown to have accuracy similar to the staggered square array model. Axisymmetric idealizations have been widely used and reported to accurately capture the real material behavior. It is reported by some authors (Socrate and Boyce (2000)) that it provides a better representation of the morphology of real materials and captures the real material behavior more accurately, especially at high volume fractions of the harder phase. The most common axisymmetric model, the stacked hexagonal array (SHA) RVE shown in Fig. 2.10 has been comprehensively used for different materials. This model is conceptually the axisymmetric analog of the idealization shown in Fig. 2.9 a. A more recent axisymmetric model developed by Socrate and Boyce (2000), the body centered cubic (V-BCC), which differs from the SHA model in only the boundary condition with neighboring cells accounting for the interaction of adjacent cells, has also been employed by several researchers.

\section{b) The Constitutive Behavior of Each Material Component:}

The mechanical behavior of each constituent of the material is required to perform modeling using the micro mechanical modeling of cells. It is not the heterogeneous material behavior, but the single component or phase behavior, which can be determined by mechanical tests. This is the only material input introduced into the micro mechanical model which adds the advantage of being able to investigate different effects of all phase or component combinations of different size, morphology, phase distribution and volume fraction by only having the constituent's mechanical behavior.

\section{c) Homogenization Method:}

The macroscopic stress components are computed as the volume average of the microscopic components according to the following equations: 


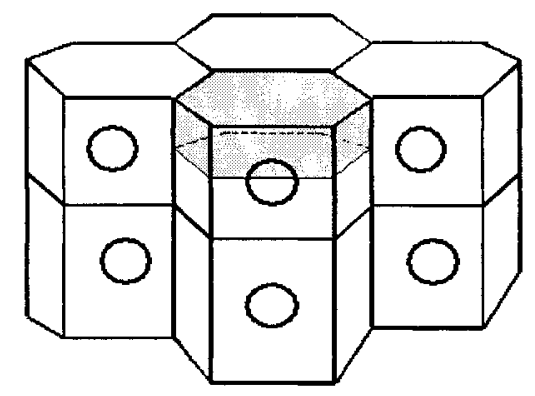

(a)

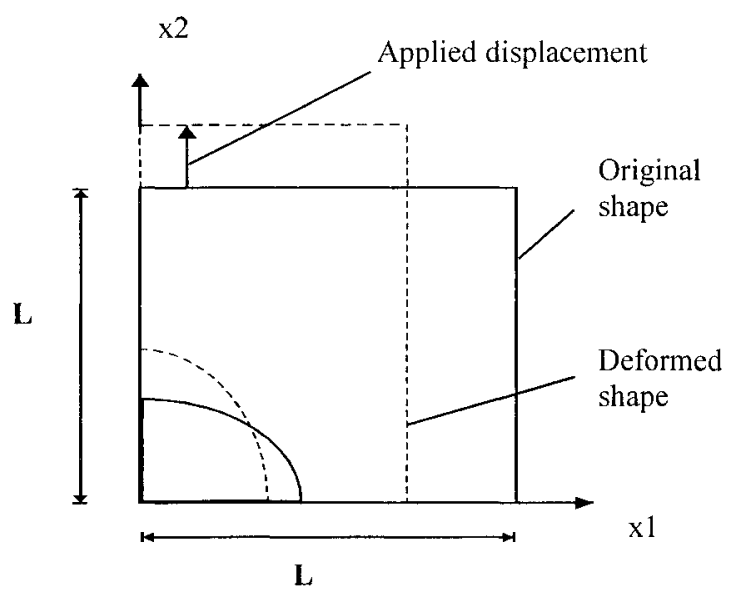

(b)

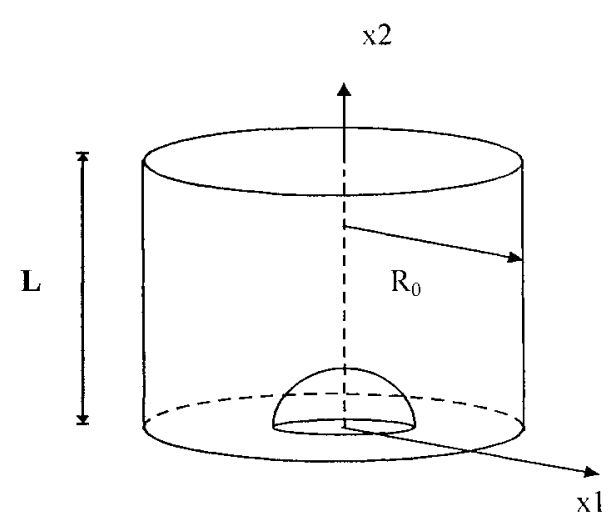

(c)

Fig. 2.10: The stacked hexagonal array (SHA) model. (a) Three dimensional array of stacked hexagonal cylinders, each containing a spherical particle (b) The deformed and undeformed shape of the RVE under axial loading (c) The SHA axisymmetric RVE cell.

$$
\begin{aligned}
& S_{i j}=\frac{1}{V} \int_{V} \sigma_{i j} d V \\
& E_{i j}=\frac{1}{V} \int_{V} \varepsilon_{i j} d V
\end{aligned}
$$


Where $S_{i j}$ and $E_{i j}$ are the macroscopic average component of stresses and strains over the microscopic volume of the micro mechanical model. The macro mechanical behavior of the aggregate is, therefore, approximated by the volume average of the micro mechanical behavior. The question of how this averaging process is performed has an obvious effect on the accuracy of the aggregate constitutive model.

Many early works in micro mechanical modeling focused on voids within a solid matrix. McClintock (1968) considered the evolution of a single cylindrical void in an infinite matrix subjected to axisymmetric loading at the remote boundary. Rice and Tracy (1969) used a variational approach to investigate the response of an isolated spherical void in an infinite medium. Both authors considered a rigid perfectly plastic material. Numerous authors have proposed improvement to these works. Gurson (1977) proposed approximate yield criteria for ductile porous media using a micro mechanical approach. Tvergaard (1981) used Gurson's yield criteria and introduced the micro mechanical modeling of cells based on a random distribution of particles that can be idealized by considering a regular three-dimensional array of hexagonal cylinders of a matrix material, each containing a spherical void or particle. The problem was further simplified by modeling axisymmetric geometry, where Tvergaard assumed that an infinite series of stacked circular cylinders containing spherical particles is a good approximation for the three-dimensional stacked hexagonal array. Symmetry arguments are then used to limit the RVE to $1 / 4$ of the axisymmetric cell.

Since Tvergaard introduced his stacked hexagonal array (SHA) model, different investigators have used it to model materials of widely different groups. Numerous investigators have shown through experimental and modeling studies that the distribution of the phases strongly affects the macroscopic material response due to the different deformation fields and localization, which arises in the matrix. A comprehensive review of this work is given by Socrate and Boyce (2000). Hung and Kinloch (1992) modeled toughening mechanisms of rubber-modified epoxy polymers using both axisymmetric and plane strain RVE's. The plane strain staggered square array model was reported to better capture the prevailing direction of shear distribution which appears to be at an angle of 
approximately $\pm 45^{\circ}$ to the direction of the applied load. This pattern was captured for different materials by many authors as reported in Socrate and Boyce (2000). However, Socrate and Boyce reported that while unit cell RVE's based on the staggered square array can effectively capture some important features of the deformation patterns, they cannot truly represent the complexity of the two-phase material and may misrepresent the effect of the actual three dimensional nature of the structure. Socrate and Boyce (2000) studied the micro mechanics of toughened polycarbonate and compared the traditional SHA model to the V-BCC model they developed which differs from the SHA model only in the boundary condition at one side of the model. They reported that at high volume fractions of the second phase, the V-BCC model accurately captures the trend of the stress-strain curves while the SHA model does not. In a more recent paper, Socrate, Boyce and Lazzeri (2001) modeled multiple crazing in high impact polystyrene using the SHA model. Tzika, Boyce and Parks (2000) studied micromechanics of deformation in particle toughened polyamides, where they used the SA RVE with boundary conditions similar to that in Tvergaard (1996) in a study of cavity growth and interaction between small and large voids. Neither the SHA model nor the V-BCC model developed by Socrate and Boyce were similar to the material phenomenon and consequently the SA model was used.

Micromechanical modeling has also been used by Christman et al. (1989), Llorca et al. (1991), Shen et al. (1994) and Shen et al. (1995) to model the behavior of metal-matrix composite materials. Christman et al. (1989) have used axisymmetric and plane strain formulations of unit cells to capture the behavior of $2124 \mathrm{Al}-\mathrm{SiC}$ composite material with whisker and spherical SiC inclusions. They also presented an investigation on the particle size distribution of whiskers after concluding that the axisymmetric and plane strain models give close responses by comparing the responses for volume percent of $13.2 \%$ of inclusions. Llorca et al. (1991) presented work on the deformation and failure of metalmatrix composites also. They investigated the effect of the shape geometry of cylindrical whiskers and spherical inclusions. They reported that the spherical shapes provided the intermediate strength and the highest ductility among the other shapes. They have also investigated the effect of the volume fraction of the inclusion and found that increasing 
the inclusion percentage reduced the ductility but only marginally affected the ultimate strength for the spherical inclusions. They have also presented the effect of the reinforcement distribution effect using the plane strain formulation for whisker shapes. Shen et al. (1995) presented similar investigations on $\mathrm{Al}-5 \%$ wt $\mathrm{Cu}$ reinforced with $20 \%$ $\mathrm{SiC}$ whiskers and particles. They ignored the microscopic (microstructural-metallurgical) details that influence the deformation behavior of the material, such as the grain size. They also studied the particle size distribution effects using only the plane strain idealizations claiming that the responses of the plane strain and axisymmetric unit cell are similar based on the work of Christman et al. (1989) and others. In addition, they presented a study of the effect of the morphology effect on the behavior of the unit cell models using cylindrical, truncated cylinder, double-cone and spherical shapes of reinforcement material and concluded that the spherical, and the truncated cylinder with sharp corners shapes give very similar responses. The plastic strain responses considered were all below $2.5 \%$, which is the fracture strain for the material they considered.

Of particular relevance for the class of materials examined in this study is work by Ishikawa et al. (2000), where the authors have developed a micro mechanical model for ferrite-pearlite dual phase steel. They used the SHA and the V-BCC models and reported that the V-BCC model could better capture the general stress-strain trend in terms of the strain hardening. Huper et al. (1999) have also developed a micro mechanical model for the ferrite-bainite dual phase steel using the plane strain idealization and reported stressstrain curves based on assumed single phase material behavior. It is shown here that the plane strain model reported by Huper et al. (1999) does not have the ability to capture the DP-steel behavior, and that the SHA model reported by Ishikawa et al. (2000) not to be accurate to capture material behavior at high $V_{m}$; to accurately capture the behavior of DP-steel. More recently, Liedl et al. (2002) modeled ferrite-martensite DP-steel using a $3 \mathrm{D}$ finite element model. They focused on predicting the $0.2 \%$ yield strength and thus reported nominal strains up to only $5 \%$ and have not considered the large plastic deformation the material experiences and confined their investigation to the initial stages of plastic deformation. They have shown that their model captures the increase in yield strength with increasing $V_{m}$. 
In this work, a micro mechanical model for the ferrite-martensite steel is developed. The importance of this type of steel prevails in its appealing combination of strength and ductility compared to other DP-steels modeled in earlier work. The improved ductility / formability of DP-steels lies in their inherent resistance to localized deformation (necking). This ability to resist necking is reflected by the attainment of large maximum uniform elongation and total elongation values due to the capacity of DP-steels to work harden at very high rates. Martensite volume fractions as high as $59 \%$ are considered. Different idealizations based on the micro mechanical modeling of cells are explored and compared. The examination of the idealized models is carried out from two perspectives in parallel. Rather than looking only at the general stress-strain trend as presented in previous studies, the deformation fields of the constituents are examined and compared to the experimental results of Shen, et al. (1976) and Rashid and Cprek (1978), as a means of assessing which idealization better describes the real material behavior. In addition, by considering aggregate strains up to $35 \%$, the model's ability to capture the gain in strength and uniform strain as the $V_{m}$ increases to a certain level and then a reduction of uniform strain and gain of strength as the $V_{m}$ is further increased, is also investigated. This tradeoff between strength and uniform strain is an extremely important factor in the optimization of microstructures for DP-steels. Further development of the model to represent the particle size distribution will be presented and experimental validation of how good the model can capture the real material behavior will be demonstrated in detail in the next Chapters.

\subsection{Failure Mechanisms in Metals:}

In quasi-static loading, materials may fail in a brittle or ductile manner. The aforementioned terms describe the mechanism of failure, which take place in any material when loaded. Each possesses some features, which makes it distinguished from the other. Ludwik was the first to describe the initiation of fracture in the middle of a round tensile 
bar. He postulated that it started by the formation of a crack due to normal stresses being highest in this region (Garrison and Moody (1987)). The spread of the crack towards the circumference was suggested to be due to the tensile radial and tangential stresses in the necked region of the test piece.

The main features in a brittle failure are (Tipper (1962), Biggs (1960), Askeland (1985) and Frechette (1990)):

1) The fracture surface is usually perpendicular to the sectioned surface.

2) Brittle fractures have a series of ups and downs forming a trend or pattern out from the origin, which give a useful guide to the direction of crack propagation.

3) There is no clear deformation of the structure as a whole.

A very strict definition of the brittle failure is given by Frechette (1990): " a brittle failure is properly distinguished from a ductile failure or viscous failure in that its fragments can be fitted together exactly, the reassembled specimen having precisely the same shape as before". This indicates that brittle failure displays essentially no plastic deformation. It is, however, not surprisingly true that all metals, whether brittle or ductile, experience some plastic deformation making the nature of failure in metals some times to be in the gray zone. A more reasonable classification of a brittle and ductile failure is to consider the mechanism through which failure occurs, which needs not only an examination of the deformation but also the examination of the microstructural details which can indicate the dominant failure mode (Biggs (1960)). 
Without roaming in the debatable zone, one can describe brittle failure as occurring with comparatively little or no plastic deformation. The initiation of a crack occurs at small flaws, which raises the stress concentration. The crack propagates at nearly the speed of sound in metals and normally along specific crystallographic planes by "cleavage" but occasionally it might take an intergranular path particularly when the inclusions weaken the grain boundaries.

Ductile failure, on the other hand, is distinguished easily by the large plastic deformation it displays. The ductile failure is more pertinent to this work and thus will be discussed in more detail. The ductile failure mechanism is characterized by three distinct mechanisms (Knot (1989), Knot (1980), Rogers (1967), Garrison and Moody (1987), Wilsdorf (1983), Gurland and Plateau (1963) and Stone et al. (1985)):

1) Formation of a free surface at an inclusion or second phase particle by either interface decohesion or particle cracking (void nucleation).

2) Growth of the void nucleated around the particles, by plastic strain and hydrostatic stress.

3) Coalescence of the growing void with neighboring voids making a sheet of voids joining together under localized strain.

Ductility is the ability of a material to deform plastically without fracture and is normally expressed by $\%$ elongation or $\%$ reduction of area at fracture. Ductility is a property of a material (Dieter (1967)), which can be viewed in different ways. Generally, ductility measurements are of interest in three main areas:

1) An indication of the extent to which a material can be deformed plastically before fracture in working operations.

2) An indication to the engineer of the ability of the material to flow plastically before fracture.

3) To be used as a guide to observe changes in purity and processing conditions (Dieter (1967)) 
Ductility has been realized to be a non-unique property as it varies with stress state, temperature and strain rate. The shape of the test specimen also influences ductility as the triaxiality which develops due to the constraint imposed on the necked region by the neighboring non-necked region, depends on the shape of the developing neck (Dieter (1967) Bridgman (1952) and Hosford and Caddell (1993)). It has been suggested that the strain to fracture will depend on the history of the state of stress and strain and not on the instantaneous state of stress as demonstrated by Bridgman (1952) where different tensile deformation histories were shown to cause a significant change in strain to fracture. Therefore the strain to fracture will change with changing testing conditions. Thus, unlike the yield stress, which can be predicted accurately for different stress states of combined stress, the reduction of area and total elongation are not true material properties but only an indicator of the fracture tendency in a certain mode of testing.

\subsubsection{Void Nucleation:}

Many attempts have been made in the past to model void nucleation, which is a rather complex problem, due to the fact that there are many features in the material that influence nucleation of cavities. It is not surprising that in doing so, it is not expected that a single criteria of critical stress or critical strain, be common to all materials since nucleation is initiated from inclusions and second phase particles (Thomason (1990)). The features of the inclusions and second phase particles such as morphology, size, distribution and cohesiveness; all play a role in expediting or slowing the void nucleation process. It has been shown and is widely accepted that ductile fracture occurs due to void growth and coalescence. On the other hand, there has been much debate as to whether or not the nucleation of cavities or voids are due to a critical strain or a critical stress as the nucleation of voids involve both stress and strain energy release. Some argued (Goods and Brown (1979)) that there must be some threshold value of far-field strain below which, there is either insufficient stress to break the interface between particles and matrix or insufficient strain energy available for the creation of new internal surfaces. Another consideration, which should be realized is that modeling void nucleation could 
be done on different levels based on the sizes of cavities and inclusions being considered (Thomason (1990), Goods and Brown (1979), Argon and Safoglu (1975) and Anderson (1995)).

A review on void nucleation, growth and coalescence models is comprehensively given in the literature and is not provided here. Excellent reviews are given by Stone et al. (1985), Garrison and Moody (1987) and Wilsdorf (1983). In the remainder of this section the major and most widely known models will be introduced, with due respect to all the works by others.

Gurland and Plateau (1963), introduced one of the first models for void nucleation at second phase particles. They assumed that when the elastic energy in the second phase particle exceeds the surface energy of the newly formed void surfaces, particle fracture would occur. They represented this for a spherical particle case and in equation form the critical stress to cause fracture was given by:

$$
\sigma_{c}=\left(\frac{\gamma E}{q^{2} d}\right)^{\frac{1}{2}}
$$

Where $d$ is the particle diameter, $E$ is the Young's modulus; $q$ is the stress concentration factor at the particle, $\gamma$ is the surface energy of particle and $\sigma$ is the critical stress for particle cracking. Later Tanaka et al. (1970) have shown that the approach of Gurland and Plateau (1963) is a necessary condition for void nucleation but not always the only condition.

Argon and Safoglu (1975), who presented the most widely known continuum void nucleation model, argued that observations by McClintock (1968) revealed that dislocation structures around inclusions of sub-micron size have shown that spacing of the surrounding dislocations in the high strain gradient zone are very much smaller than the particle diameter, which means at least for large particles, a continuum analysis of 
deformation is the most proper. In addition, they concluded based on previous published work that for small particles $\left(<<250 \mathrm{~A}^{\circ}\right)$ the energy criterion (requirement) may not be satisfied for the separation of inclusion and matrix (void nucleation) even when the local stress reaches the interfacial strength and thus they focused on large particles in their investigation. Argon and Safoglu (1975), based on finite element analysis, argued that the interfacial stress for a cylindrical particle is equal to the sum of the hydrostatic stress and the effective stress. They defined the decohesion stress as:

$$
\sigma_{c}=\sigma_{e}+\sigma_{h}
$$

Where $\sigma_{e}$ is the effective stress given by:

$$
\sigma_{e}=\frac{1}{\sqrt{2}}\left[\left(\sigma_{1}-\sigma_{2}\right)^{2}+\left(\sigma_{2}-\sigma_{3}\right)^{2}+\left(\sigma_{3}-\sigma_{1}\right)^{2}\right]^{\frac{1}{2}}
$$

And $\sigma_{h}$ is the mean or hydrostatic stress given by:

$$
\sigma_{h}=\frac{\sigma_{1}+\sigma_{2}+\sigma_{3}}{3}
$$

Where $\sigma_{1}, \sigma_{2}$ and $\sigma_{3}$ are the particle principal stresses. From equation 2.13 it can be seen that the interfacial stress will increase with strain hardening and with hydrostatic stress. This is in general agreement with Bridgman (1952) experiments where he has shown that negative hydrostatic stress increases the ductility.

Beremin (1981) studied cavity formation from inclusions in ductile fracture of A508 steel. He concluded that the Argon and Safoglu criteria can better correspond to experimental observations if the equation for the critical stress is modified to: 


$$
\sigma_{c}=\sigma_{h}+c\left(\sigma_{e}-\sigma_{y s}\right)
$$

Where $\sigma_{y s}$ is the yield stress and $c$ is a fitting parameter.

As a most widely known dislocation based model, Goods and Brown (1979) presented another model based on smaller particle sizes. A necessary condition for the nucleation of a microvoid, by decohesion of the particle-matrix interface, is that the elastic strain energy released by the particle must be greater than or equal to the energy created by the new surface(s) (Goods and Brown (1979), Thomason (1990), Anderson (1995), Argon and Safoglu (1975), Gurland and Plateau (1963), Garrison and Moody (1987), Wilsdorf (1983), Stone et al. (1985) and Tanaka et al. (1970)). The local stress caused by dislocation pile-up due to a far field stress which adds to the stress requirement for particle matrix decohesion has been shown by Brown and Stobbs (1976) to be expressed by:

$$
\sigma_{L}=\alpha \mu b(\rho)^{\frac{1}{2}}
$$

Where $\alpha$ is a constant between 0.14 and $0.33, \mu$ is the shear modulus and $\rho$ is the dislocation density around a particle which has been approximated by Brown and Stobbs by:

$$
\rho=1.7\left(\frac{\varepsilon_{1}}{r b}\right)
$$

Where $r$ is the particle size, $b$ is the magnitude of Burger's vector and $\varepsilon_{1}$ is the maximum remote normal strain. Thus, equation 2.16 can be expressed in the following form: 


$$
\sigma_{L}=1.3 \alpha \mu\left(\frac{\varepsilon_{1} b}{r}\right)^{\frac{1}{2}}
$$

Because of the plastic constraints caused by the particle, the local stress at the interface $\sigma_{L}$ is increased and was determined by Brown and Stobbs to be 4.2 times greater. Thus, the elevated local stress, $\sigma_{k}$, on the particle interface is given by (Goods and Brown (1979)):

$$
\sigma_{E}=5.4 \alpha \mu\left(\frac{\varepsilon_{1} b}{r}\right)^{\frac{1}{2}}
$$

And the total maximum interface stress, $\sigma_{T}$, is given by:

$$
\sigma_{T}=\sigma_{E}+\sigma_{h}
$$

Where $\sigma_{h}$ is the hydrostatic stress. Hence the critical stress, $\sigma_{c}$ for microvoid nucleation is given by:

$$
\sigma_{c}=\sigma_{T}+\sigma_{1}
$$

Where $\sigma_{1}$ is the maximum remote normal stress. This model is called the Goods and Brown model. It is evident in this model that as the particle size decreases the local stress requirement for decohesion increases and thus void nucleation will be more difficult to form with the small particles than with larger ones. On the other hand, Argon and Safoglu (1975) and Beremin (1981) criteria indicate that the critical stress is independent of the particle size. 
The volume fraction of the voids, $f_{v}$, in the process of deformation is not expected to be constant but increases due to the growth of existing voids and nucleation of the new voids. The total rate of change is given as (Tvergaard (1982)):

$$
d f_{v}=d f_{m u c l .}+d f_{g r}
$$

In numerical modeling the void nucleation is generally written in a form which consist of two parts, namely, stress controlled and strain controlled quantities as follows:

$$
d f_{\text {macl. }}=A d \varepsilon_{e}{ }^{p}+B\left(d \sigma_{h}+d \sigma_{e}\right)
$$

Where $A$ and $B$ are strain and stress controlled void nucleation intensity parameters, respectively, $\sigma_{e}$ is the effective (von Mises) stress, $\sigma_{h}$ is the hydrostatic stress and $\varepsilon_{e}{ }^{\prime \prime}$ is the effective plastic strain. The strain controlled part in equation 2.24 is based on equation 2.20 and the stress controlled part is based on equation 2.13. Three void nucleation laws are possible, cluster nucleation, continuous nucleation and statistical void nucleation as shown in Fig. 2.11 where $S$ stands for stress or strain quantity (Zhang (2001)). Fig. 2.11a depicts the cluster nucleation model where it is assumed that voids nucleate when some critical condition is reached and usually at the beginning of plastic deformation. In this model, the intensity factor is taken as $f_{0}$. The second law, Fig. $2.11 \mathrm{~b}$ is called the continuous model.

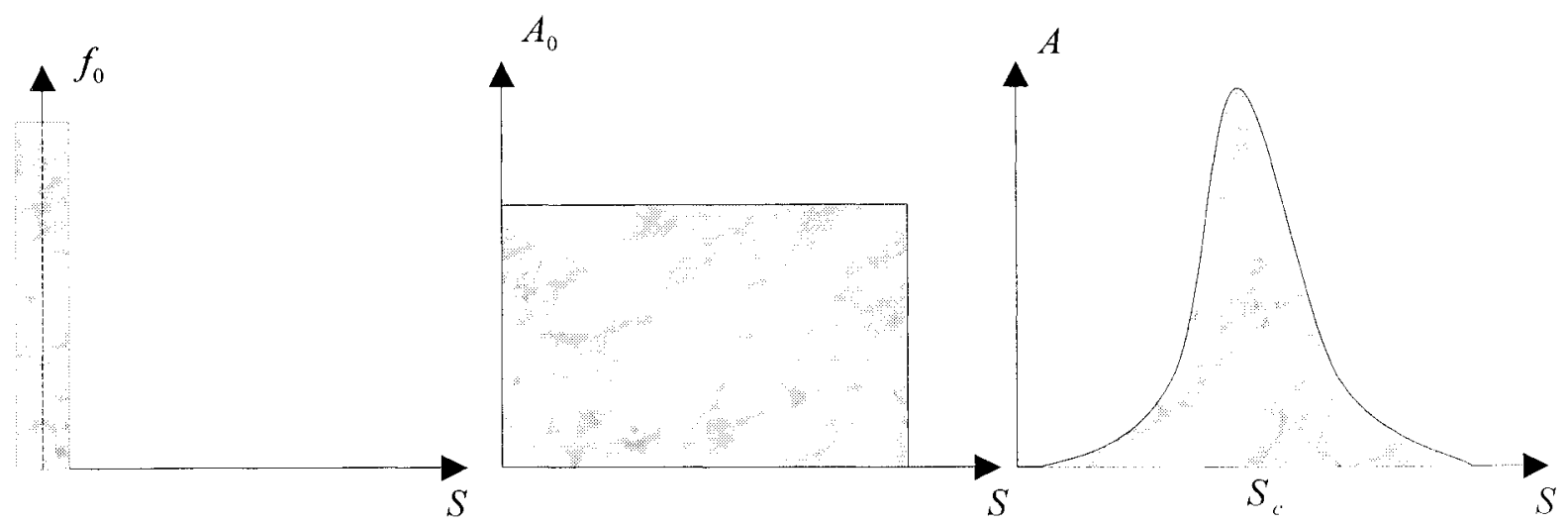

Fig. 2.11: Void nucleation models (a) cluster nucleation, (b) Continuous nucleation and (c) Statistical nucleation model (adopted from (Zhang (2001)). 
Some materials exhibit increasing voids with increasing plastic strain (Gurland (1972)) which indicates a strain intensity parameter of the form, $A_{0}=d f_{v} / d \varepsilon_{e}$. The last model as depicted in Fig. 2.11c, indicates that most voids nucleate around a critical quantity, $S_{c}$, which can be stress or strain. Chu and Needleman (1980) proposed the following intensity parameter for strain controlled intensity factor (ABAQUS (2000)):

$$
A=\frac{f_{N}}{S_{N} \sqrt{2 \pi}} \exp \left[\frac{-1}{2}\left(\frac{\varepsilon_{e}{ }^{p}-\varepsilon_{N}}{S_{N}}\right)^{2}\right]
$$

where $f_{N}$ is the volume fraction of particles or inclusion, $\varepsilon_{N}$ is the mean nucleation burst strain and $S_{N}$ is the corresponding standard deviation. For a stress controlled intensity factor, the expression they recommended was:

$$
B=\frac{f_{N}}{S_{N} \sqrt{2 \pi}} \exp \left[\frac{-1}{2}\left(\frac{\sigma_{e}+\sigma_{h}-\sigma_{N}}{S_{N}}\right)^{2}\right]
$$

where $\sigma_{N}$ is the mean nucleating stress and $\sigma_{h}$ is the mean or hydrostatic stress and $\sigma_{e}$ is the effective stress.

The void growth rate is based on the law of conversation of mass and is expressed as:

$$
d f_{g r}=\left(1-f_{v}\right) \operatorname{trace}\left(d \varepsilon_{i j}^{p}\right)
$$

where $\varepsilon_{i j}{ }^{p}$ is the plastic strain tensor. 


\subsection{2: Void Growth and Coalescence:}

In polycrystalline metals of very high purity, microvoids are absent due to the absence of void nucleation sites such as second phase particles and inclusions. In such materials, fracture using a tension test occurs virtually by $100 \%$ reduction in area of the external neck formed due to plastic instability. Once the ultimate strength of the material is reached (point of plastic instability) on a nominal stress-strain curve, any further increase of the stress causes the weakest part of the specimen to externally neck. However, all engineering metals and alloys contain inclusions and second phase particles to some degree, which lead to microvoid nucleation and growth, which causes premature material failure.

Once the voids nucleate, further plastic strain and hydrostatic stress causes the void to grow and eventually coalesce. The mechanism of void growth and coalescence can be illustrated as shown in Fig. 2.12. Once the voids nucleate, they grow by further plastic strain, independently, each under the influence of local stresses dominated by the morphology and size of the inclusions or second phase particles which means that the growth of each void may vary from one void to another. Upon further increase in the plastic strain, neighboring voids interact and plastic strain due to each may overlap which may increase total strain in the vicinity of each void. Local necking of the ligaments between voids takes place enhancing the formation of a sheet of voids, which eventually coalesce causing fracture. Void growth and coalescence is strongly dependent on the extent of stable void growth before void coalescence. An estimated $87 \%$ of energy consumed during "dimpled fracture" of high strength steel is absorbed during void growth (Stone et al. (1985)).

Unlike void nucleation and growth which happens in a stable manner, void coalescence occurs rapidly and catastrophically which makes it the most difficult stage of void evolution to investigate. Void coalescence can occur by void impingement or by the void-sheet process. The formation of void sheets aborts the other stable void growth process and thus diminishes ductility and causes the rapid catastrophic fracture. Although 
void coalescence is the fastest stage of void evolution, unlike void nucleation and growth, it occurs in several stages. First the strain localization occurs, then a sheet of voids form, which interact mostly, and finally ligaments between the voids neck. At the same time this process takes place, there are other voids in the localized strain areas, which form and grow which makes it even more difficult to investigate. Adding to the above, different inclusions and particle shapes and sizes form voids at different stages and at different growth rates and interaction mechanism with other voids.

There are a number of mathematical models for the growth and coalescence of microvoids. Bridgman (1954) concluded from his study of metals under high pressure that there is generally, no acceptable criteria of fracture based on stress alone. Cockroft (1967) argued after discussing that neither stress nor strain could be used alone as fracture criteria, that successful fracture criteria must take into account stress and strain. Rice and Tracy (1969) developed a model considering a single void in an infinite solid, subjecting the void to remote or far field normal stresses and strains. They presented an equation, which can approximate the void growth of the following form:

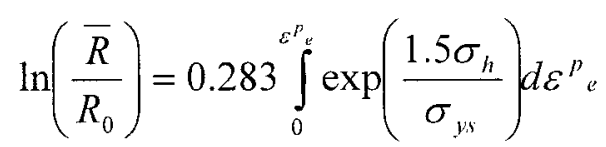

Where $\vec{R}$ is the mean radius and $\varepsilon^{p}{ }{ }$ is the effective plastic stress. The Rice and Tracy model is based on a single void and thus does not account for void interaction nor can it predict failure, as it does not account for softening resulting from void growth. Gurson (1977) analyzed plastic flow in a void containing material based on the work of Rice and Tracy (1969) and McClintock (1968). In the model by Gurson, the softening effect due to the presence of voids was reflected in a yield function. He carried out a rigid-plastic upper bound analysis to obtain his yield function. Notably, the classical yield criteria (von Mises) does not account for the presence of voids. This was because of the fact that 
the variation of the hydrostatic stress did not have any influence on the magnitude of the slip-plane shear stress, and thus was omitted from the J2 flow rule. Contrary to this, Bridgman (1952) was the first to show that the hydrostatic stress had considerable effect on increasing the failure strain when positive pressure is used to trap the void (not to grow). Among the different void shapes Gurson investigated, the spherical void, which was the more realistic void shape corresponding to real materials, has received most attention in the subsequent development of Gurson's model. Gurson (1977) in his model accounted for hydrostatic stress by introducing a strain softening term in the yield function, which has the following form:

$$
\Phi=\left(\frac{\sigma_{e}}{\sigma_{y}}\right)^{2}+2 f_{v} \cosh \left(\frac{3 \sigma_{h}}{2 \sigma_{y}}\right)-\left(1+f_{v}^{2}\right)=0
$$

Where $f_{v}$ is the void volume fraction, $\sigma_{e}$ is the effective stress, $\sigma_{h}$ is the hydrostatic stress and $\sigma_{y}$ is the yield stress of the fully dense matrix material. This equation reduces to the von Mises yield function when $f_{v}$ is set to zero. From equation 2.29 it is obvious that the material yielding is coupled with a damage variable, $f_{v}$, and the hydrostatic stress. The yield surface in Gurson's model decreases with the increase of $f_{v}$ until the complete loss of the load carrying capacity. 


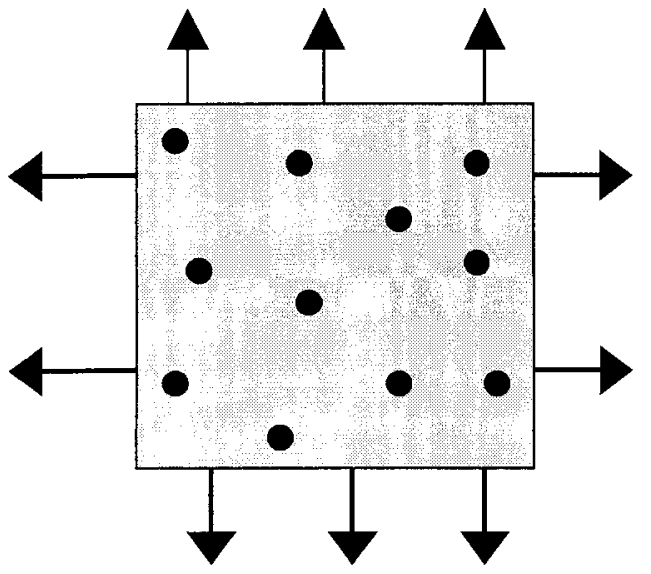

a) Inclusion in a ductile matrix.

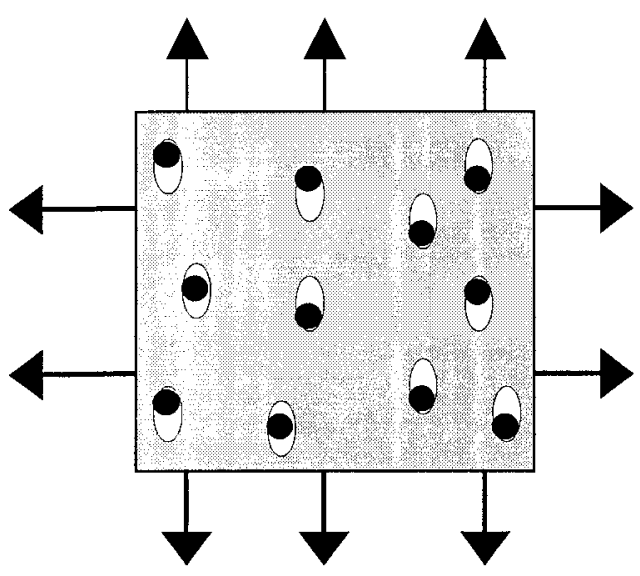

c) Void growth.

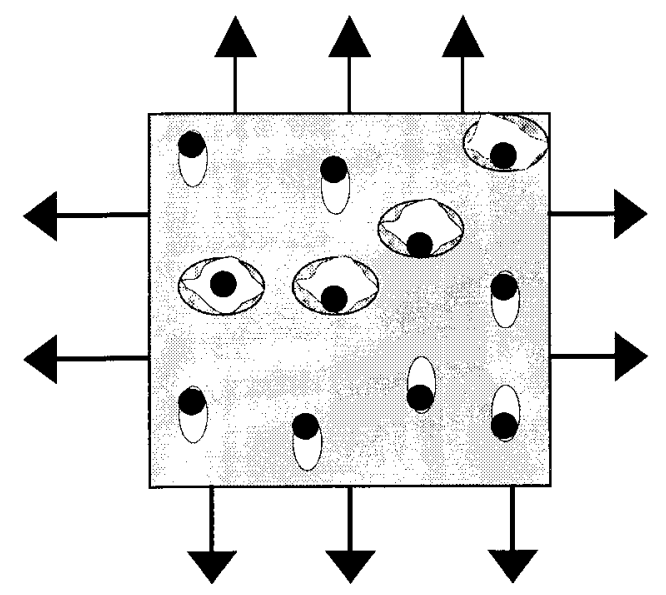

e) Necking between ligaments.

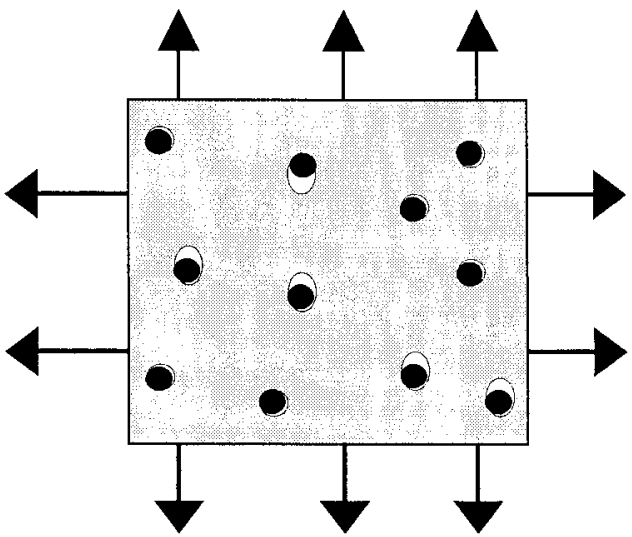

b) Void nucleation.

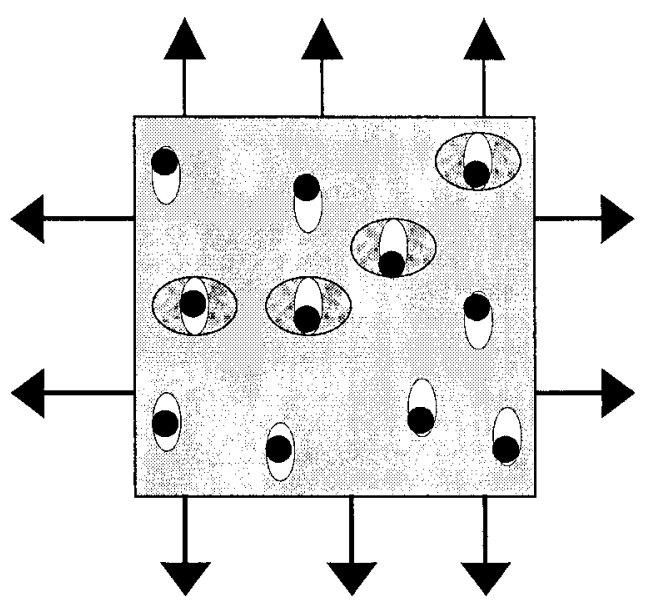

d) Strain localization.

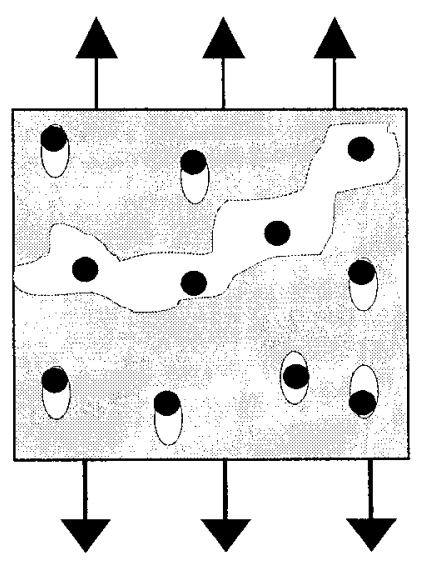

f) Void coalescence and fracture.

Fig. 2.12: Void nucleation, growth and coalescence in ductile metals [adapted from Anderson (1995) and Thomason (1990)] 
Gurson's model has been reported to greatly overpredict the elongation to failure in real materials. This is basically due to the unrealistic phenomena it predicts which is that complete loss of the load carrying capacity occurs when the void volume grows to $f_{v}=100 \%$. Tvergaard $(1981,1982)$ in a study based on Gurson's model compared predictions of an axisymmetric model and introduced his micro mechanical cell model based on the stacked hexagonal array (SHA). He calibrated Gurson's yield function by introducing the parameters $q_{1}, q_{2}$ and $q_{3}$ changing the yield function to:

$$
\Phi=\left(\frac{\sigma_{c}}{\sigma_{y}}\right)^{2}+2 f_{v} q_{1} \cosh \left(\frac{3 q_{2} \sigma_{h}}{2 \sigma_{y}}\right)-\left(1+q_{3} f_{v}^{2}\right)=0
$$

Typically $q_{3}$ is taken as $q_{1}^{2}$ and the equation becomes (ABAQUS (1998)):

$$
\Phi=\left(\frac{\sigma_{e}}{\sigma_{y}}\right)^{2}+2 f_{v} q_{1} \cosh \left(\frac{3 q_{2} \sigma_{h}}{2 \sigma_{y}}\right)-\left(1+\left(q_{1} f_{v}\right)^{2}\right)=0
$$

Tvergaard suggested calibrating parameters $q_{1}=1.5$ and $q_{2}=1$ (Tvergaard $(1981,1982)$ ) to better capture experimental results. Faleskog et al. (1998) and Geo et al. (1998) presented a procedure for choosing the proper calibration parameters $\left(q_{1}\right.$ and $\left.q_{2}\right)$ depending on the strain hardening rate of metal materials using a three dimensional model for moderate and high strain hardening materials as the parameters suggested by Tvergaard $(1981,1982)$ and Tvergaard and Needleman (1984) were not adequately capturing the real material behavior at different strain hardening rates. The optimal parameters they presented are summarized in table 2, where $\mathrm{n}$ is the strain hardening index and $\sigma_{0}$ is the yield strength. 


\begin{tabular}{|c|c|c|c|c|c|c|}
\hline \multirow{2}{*}{ Hardening, $\mathrm{n}$} & \multicolumn{2}{|c|}{$\sigma_{0} / \mathbf{E}=\mathbf{0 . 0 0 1}$} & \multicolumn{2}{c|}{$\sigma_{0} / \mathbf{E}=\mathbf{0 . 0 0 2}$} & \multicolumn{2}{c|}{$\sigma_{0} / \mathbf{E}=\mathbf{0 . 0 0 4}$} \\
\cline { 2 - 7 } & $\mathrm{q}_{1}$ & $\mathrm{q}_{2}$ & $\mathrm{q}_{1}$ & $\mathrm{q}_{2}$ & $\mathrm{q}_{1}$ & $\mathrm{q}_{2}$ \\
\hline 0.025 & 1.88 & $\mathbf{0 . 9 5 6}$ & 1.84 & $\mathbf{0 . 9 7 7}$ & 1.74 & 1.013 \\
\hline 0.050 & 1.63 & 0.950 & 1.57 & 0.974 & 1.48 & 1.013 \\
\hline 0.075 & 1.52 & 0.937 & 1.45 & 0.960 & 1.33 & 1.004 \\
\hline 0.100 & 1.58 & 0.902 & 1.46 & 0.931 & 1.29 & 0.982 \\
\hline 0.150 & 1.78 & $\mathbf{0 . 8 3 3}$ & 1.68 & 0.856 & 1.49 & 0.901 \\
\hline 0.200 & 1.96 & 0.781 & 1.87 & 0.800 & 1.71 & 0.836 \\
\hline
\end{tabular}

Table 2.1: Calibrated values (optimal) of $q_{1}$ and $q_{2}$ of the Gurson Tvergaard yield function (Faleskog et al. (1998))

The calibrations proposed after Gurson presented his model, intensify the effect of the hydrostatic stress and the void volume fraction, but does this through all the deformation process and not only after necking. In addition, the Gurson model was originally derived based on homogeneous matrix deformation. Therefore, even with the calibration and improvements cited above, it can capture the nucleation and growth of voids but still it has no intrinsic ability to predict coalescence e.g: transition from homogeneous to localized deformation mode. As mentioned earlier, coalescence takes place when the ligaments between the voids neck, making up a dense carpet of voids, which coalesce, and cause rupture.

This point was realized by many researchers (Tvergaard and Needleman (1984) and Koplik and Needleman (1988)) and suggested a further modification to Gurson's model by replacing $f_{v}$ with an effective void volume fraction $f_{\mathrm{v}}^{*}$ given by: 


$$
f_{v}^{*}= \begin{cases}f_{v} & \text { for } f_{v} \leq f_{v, c} \\ f_{v, c}-\frac{f^{*}{ }_{v, U}-f_{v, c}}{f_{v, F}-f_{v, c}}\left(f_{v}-f_{v, c}\right) & \text { for } f_{v}>f_{v, c}\end{cases}
$$

Where $f_{v, c}$ is the critical void volume fraction, $f_{v, f}$ is the void volume fraction at final fracture and $f_{v, \ell}=\frac{1}{q_{1}}$. This effective void volume fraction imposes an abrupt increase in the loss of load carrying capacity when the void volume fraction reaches a critical value, $f_{v, c}$, accelerating the onset of plastic instability. One of the disadvantages of the revised Gurson model is that it contains numerous adjustable parameters.

Thomason (1990) developed a simple model, which predicts internal necking of the microvoids. After a detailed analysis of the mechanics of ductile fracture by void coalescence he found that the localization mode of deformation could be well described by a plastic limit load. His model states that coalescence occurs when the net section stress in the ligaments between the voids reaches a critical value, $\sigma_{n(c)}$. Fig. 2.13 illustrates a two-dimensional case, with cylindrical voids of dimensions $2 \mathrm{a}$ and $2 \mathrm{~b}$ and intervoid distance of $2 \mathrm{~d}$ subjected to plain strain loading. The load criteria is expressed as:

$$
\sigma_{n(c)} \frac{d}{d+b}>\sigma_{1}
$$

And the fracture occurs when:

$$
\sigma_{1}=\sigma_{n(c)} \frac{d}{d+b}
$$


Where $\sigma_{1}$ is the far field maximum principal stress.

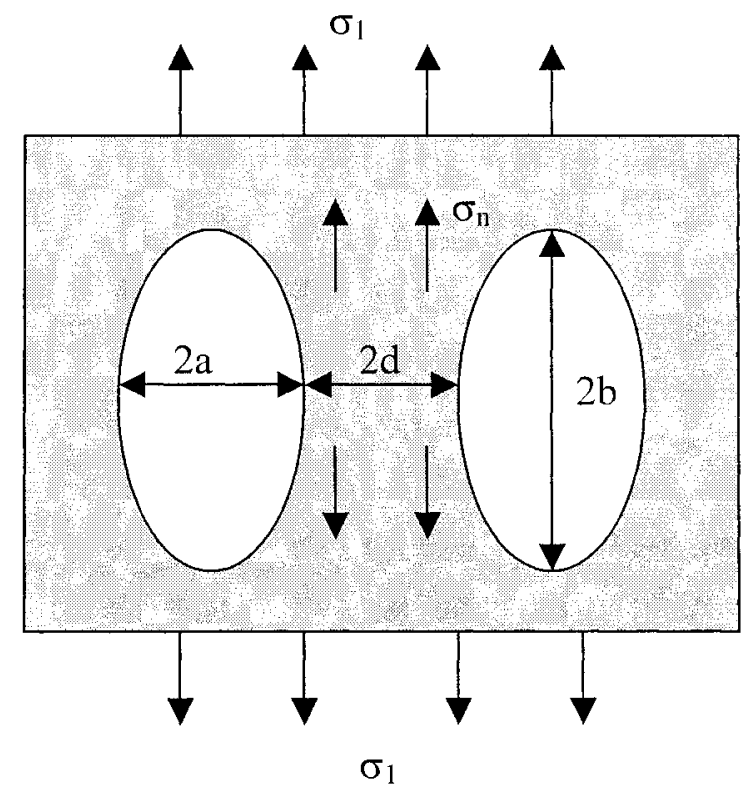

Fig. 2.13: Ductile Crack Growth (Adapted from (Anderson (1995)) 


\section{CHAPTER}

\section{ELASTOPLASTIC MATERIAL MODELING}

Any material body deforms when it is subjected to external forces. The elastic-plastic deformation of solids can be studied from different viewpoints. Generally, two categories emerge from the vast content of elastoplastic materials. One is the phenomenological approach within the framework of continuum mechanics (also referred to as macro elastoplasticity) and the other category, which is more physically motivated is to deal with the physical background of the material (also referred to as micro elastoplasticity). In this chapter, the fundamentals of the macro elastoplastic material behavior and modeling will be introduced. Although the present work deals with material micromechanically, the phenomenological approach is needed to address material behavior described for the constituents of the microstructure as each part is considered as an elastoplastic material.

The elastic part of the deformation is generally separated from the plastic part by the yielding point. When a material is deformed, it stretches elastically; and upon removing 
the loading within the elastic regime, it recovers its original shape and dimensions. On the other hand, if the material is stretched beyond it's yielding limit, it undergoes permanent plastic deformation.

The starting point in both the theory of elasticity and theory of plasticity is the construction of explicit relationships between stress and strain for uniform states of combined stresses. The equations that describe these relationships are known as constitutive equations. In the case of linear elasticity theory, for example, the constitutive relationships are known as Hooke's law. It is essential for accurate prediction of the stress and strain to know the point at which the body changes from elastic to plastic deformation; the yield point. The relationship, which determines the point of transformation from elastic to plastic deformation, is called a yield criterion. The constitutive equations, yield criteria, and equilibrium equations enable us to calculate strain for non-uniform states of stress (Davis and Selvadurai (2002) and Lubliner (1990)).

\subsection{Uniaxial Stress-Strain Loading:}

Before getting into the main thrust of this chapter, it is important to consider first the data obtained from a simple tensile test.

\subsubsection{Definition of Stress and Strain:}

Considering the tensile loading of a specimen of material with original cross section area $\mathrm{A}_{0}$ and length $\mathrm{L}_{0}$ as shown in Fig. 3.1, the nominal stress and strain are defined as: 

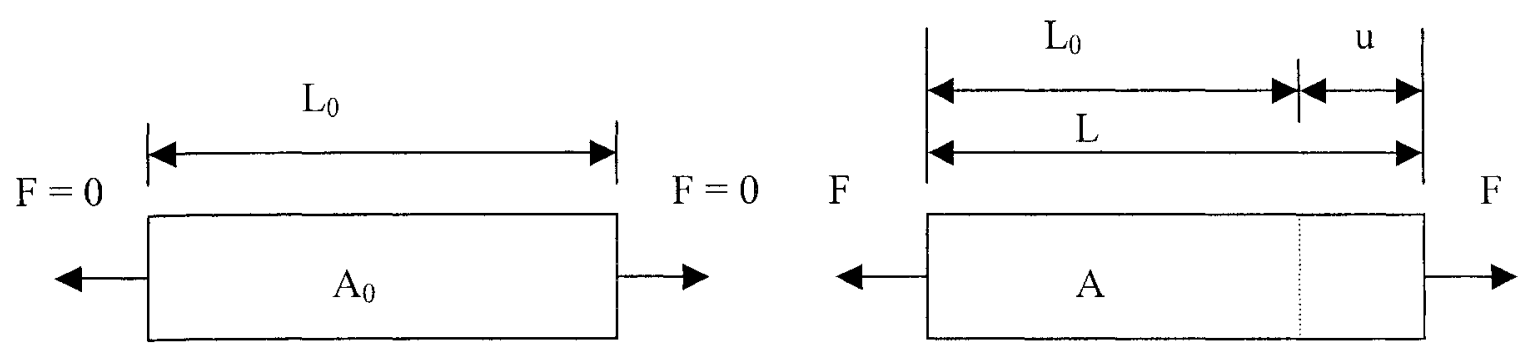

Fig. 3.1: Loading of a round tensile bar.

$$
\begin{gathered}
\sigma_{n}=\frac{F}{A_{0}} \\
e=\frac{u}{L_{0}}=\frac{L-L_{0}}{L_{0}}=\frac{L}{L_{0}}-1
\end{gathered}
$$

The true stress is defined as:

$$
\sigma=\frac{F}{A}
$$

and true strain is defined as:

$$
\varepsilon=\int_{L_{0,0}}^{L} \frac{d L}{L}=\ln \left(\frac{L}{L_{0}}\right)
$$

Where $L$ and $A$ are the instantaneous length and cross sectional area of the tensile specimen respectevely. Relationships between $\sigma_{n}, e$ and $\sigma, \varepsilon$ can be determined fairly easily provided we assume constancy of volume. This assumption is valid in the elastic regime if Poission's ratio $v=1 / 2$. However, this assumption is more valid when plastic deformation is much greater than elastic deformation which can be also seen from the relation for the dilatancy, $\Theta$ : 


$$
\Theta=\frac{\sigma_{h}}{K_{v}}
$$

Where $K_{\nu}$ is the bulk modulus $\left[K_{\nu}=\frac{E}{3(1-2 v)}\right]$ and $\sigma_{h}$ is the mean stress $\left[\sigma_{h}=\frac{\sigma_{i i}}{3}\right]$.

If $v=1 / 2$ then $\Theta=0$ which means that the material is incompressible (Padmanabhan et al. (2001)). In the case where the constant volume assumption holds, the following relationships can be shown to be true for uniform strain:

$$
\begin{gathered}
\sigma=\sigma_{n}(1+e) \\
\varepsilon=\ln \left(\frac{L}{L_{0}}\right)=\ln (1+e)
\end{gathered}
$$

It has to be stressed that these relations are valid for the range of stresses and strains below the ultimate stress (point of instability - necking) where the deformation changes from uniform to nonuniform deformation. After necking, equations 3.6 and 3.7 are not valid. The length changes are localized in the neck; so the nominal strain (e), which involves a measurement using the entire gage section, cannot be used to calculate the true strain, $\varepsilon$. In addition, the expression $\left(\sigma=\frac{F}{A}\right)$ gives the average true stress in the neck for the direction of loading. This is no longer the effective stress since the stress state in the neck is triaxial (ASM (1992)).

\subsubsection{Instability (Necking) of a Tensile Specimen:}

For a round bar tensile specimen, at the instability (maximum stress) the change in force becomes zero owing to the inflection point it passes through due to the change from the hardening to softening regimes which can be denoted as:

$$
d F=0
$$


From this it can easily be seen that:

$$
d F=d(\sigma A)=A d \sigma+\sigma d A=0
$$

Under the assumption of material incompressibility, then $(A L=$ constant $)$ :

$$
A d L+L d A=0
$$

Which gives:

$$
\frac{d A}{A}=-\frac{d L}{L}=-d \varepsilon
$$

From 3.8 and 3.10, we can obtain the following, which hold at the point of instability:

$$
\frac{d \sigma}{\sigma}=d \varepsilon \text { or } \frac{d \sigma}{d \varepsilon}=\sigma
$$

\subsubsection{Effective (Equivalent or Representative) Stress and Strain:}

Most stress-strain data is obtained from uniaxial tensile and compressive tests. It is difficult to see how this information can be used when a material is subjected to a combined sate of stress. Therefore, it is more convenient to deal with effective stresses and strains, which will be shown shortly.

Considering a material subjected to a combined state of stress as shown in Fig. 3.2, one possible way to express the stress and strain in a global manner is to consider volumetric plastic work, which can be defined as:

$$
d w=\sum_{i=1}^{3} \sigma_{i} d \varepsilon_{i}
$$




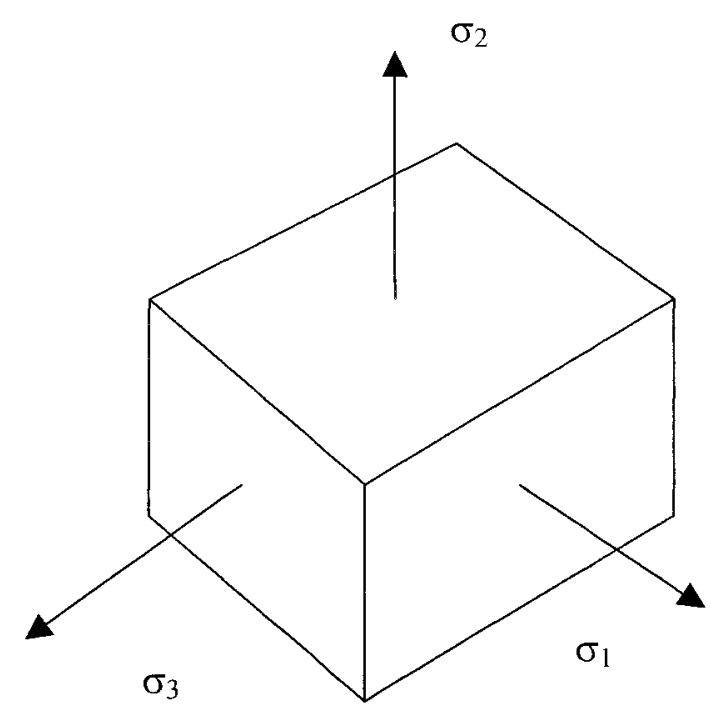

Fig. 3.2: Material subjected to combined state of stress.

where $d w$ is the specific plastic work (work done per unit volume). In the case above based on the von Mises yield criteria, the material yields when the stress reaches a yield value equal to:

$$
Y=\frac{1}{\sqrt{2}}\left[\left(\sigma_{1}-\sigma_{2}\right)^{2}+\left(\sigma_{2}-\sigma_{3}\right)^{2}+\left(\sigma_{3}-\sigma_{1}\right)^{2}\right]^{\frac{1}{2}}
$$

Which suggests that we define our effective stress to be:

$$
\sigma_{e}=\frac{1}{\sqrt{2}}\left[\left(\sigma_{1}-\sigma_{2}\right)^{2}+\left(\sigma_{2}-\sigma_{3}\right)^{2}+\left(\sigma_{3}-\sigma_{1}\right)^{2}\right]^{\frac{1}{2}}
$$

The effective version of the specific plastic work presented in 3.12 can be expressed in the form:

$$
d w=\sigma_{e} d \varepsilon_{e}
$$

From equations 3.14 and 3.15 the effective strain, can be easily determined which takes the form: 


$$
\varepsilon_{e}=\sqrt{\frac{2}{9}}\left[\left(\varepsilon_{1}-\varepsilon_{2}\right)^{2}+\left(\varepsilon_{2}-\varepsilon_{3}\right)^{2}+\left(\varepsilon_{3}-\varepsilon_{1}\right)^{2}\right]^{\frac{1}{2}}
$$

\subsubsection{Work Hardening:}

Work hardening or strain hardening is an ever-increasing load requirement for continued deformation beyond the yield point. Most metals have this property. To get more strain or deformation we have to apply more load and not a constant load. It is worth shedding light on the phenomenological treatment of strain hardening which will be the subject of this section.

With many ductile metals that have not been cold worked prior to the tensile test, the behavior from initial yield to ultimate load is adequately described by a power law of the form:

$$
\sigma=K \varepsilon^{n}
$$

Where $\varepsilon$ is the induced true strain and $K$ and $n$ are constants for a material. For a material, which follows a power law description and tested in a uniaxial tension test, the onset of plastic instability as deduced in equation 3.11 can be worked out easily:

From equation $3.11, \because \frac{d \sigma}{d \varepsilon}=\sigma \quad \Rightarrow \quad \frac{d \sigma}{d \varepsilon}=n K \varepsilon^{n-1}=\sigma=K \varepsilon^{n}$

Reducing the terms gives, at the point of instability: 


$$
n=\varepsilon
$$

The stress and strain in the above analysis are the effective stress and strain, as in the uniaxial tensile test of a round bar, the axial stress and strain work out to be the effective ones, this can be seen from equation 3.14 in the last chapter. In certain analysis, it is worth simplifying any problem from the real three dimensional view to a twodimensional view, if the simplification does not cause significant loss of accuracy of the results. This can only be done in special cases with certain geometrical features or characteristics and loading conditions, which enable simplifying assumption.

Plane stress and plane strain are two cases, which reduce the three-dimensional case to a two-dimensional case. The plane strain approximation is appropriate for long cylinders or bars, where the strain in the relatively long direction is assumed to be zero. Thus the nonzero strains occur only in the other two directions. The plane stress case is an appropriate assumption for thin sheets where the plate is subjected to load in the plane of the plate. If the plate is thin compared to it's width, the stress in the direction normal to the plate is assumed to be zero and so are the shear stresses.

The prediction of the onset of necking for a material following the power law rule can be worked out for a simplified plane strain case also. Ignoring the elastic strains, the equations are developed as follows: 


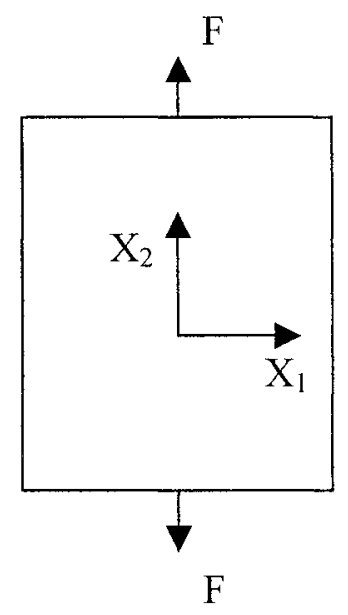

Fig. 3.3: Plane strain case, of a long bar subjected to loading as shown.

$F=\sigma_{22} A$ and thus differentiating we get $\quad d F=\sigma_{22} d A+A d \sigma_{22}$

Since at necking we have $d F=0, \Rightarrow \quad \frac{d \sigma_{22}}{\sigma_{22}}=-\frac{d A}{A}$

Suppose $A=w t$, where $\mathrm{w}$ is the width and $\mathrm{t}$ is the thickness then

$$
\begin{gathered}
d A=t d w \\
\therefore \quad \frac{d \sigma_{22}}{\sigma_{22}}=-\frac{d A}{A}=-\frac{t d w}{w t}=-\frac{d w}{w}=-d e_{11} \\
\therefore \frac{d \sigma_{22}}{\sigma_{22}}=d e_{22}=-d e_{22} \Rightarrow \frac{d \sigma_{22}}{d e_{22}}=\sigma_{22}
\end{gathered}
$$

The effective (equivalent) incremental strain can thus be calculated and found to be:

$$
\begin{gathered}
d e_{e}=\frac{\sqrt{2}}{3}\left[\left(2 d e_{22}\right)^{2}+\left(d e_{22}\right)^{2}+\left(d e_{22}\right)^{2}\right]^{1 / 2} \\
\therefore d e_{e}=\frac{2}{\sqrt{3}} d e_{22}
\end{gathered}
$$


For the plane strain case $\left(\varepsilon_{33}=0\right), \sigma_{33}$ is well approximated by the intermediate value of the other normal stresses $\left(\sigma_{33}=\frac{\sigma_{11}+\sigma_{22}}{2}\right)$ after a plastic strain a few times the strain at yield (Hill (1950) and Lubliner (1990)). Since $\sigma_{11}=0, \sigma_{33}$ is taken to be $\frac{\sigma_{22}}{2}$

$$
\begin{array}{cl}
\therefore & \sigma_{e}=\frac{1}{\sqrt{2}}\left[\left(-\sigma_{22}\right)^{2}+\left(\frac{\sigma_{22}}{2}\right)^{2}+\left(\frac{\sigma_{22}}{2}\right)^{2}\right]^{1 / 2} \\
\therefore & \sigma_{e}=\frac{\sqrt{3}}{2} \sigma_{22} \\
\therefore & d \sigma_{e}=\frac{\sqrt{3}}{2} d \sigma_{22}
\end{array}
$$

From 3.19 and 3.20 the following can be determined:

$$
\frac{d \sigma_{22}}{d e_{22}}=\frac{4}{3} \frac{d \sigma_{e}}{d e_{e}}
$$

For the same condition as in the cylindrical rod from equation 3.11, the instability condition for the $\mathrm{x}_{2}$ direction is:

$$
\frac{d \sigma_{22}}{d e_{22}}=\sigma_{22}
$$

Thus for plane strain case this condition will be:

$$
\frac{4}{3} \frac{d \sigma_{e}}{d e_{e}}=\frac{2}{\sqrt{3}} \sigma_{e}
$$

For a power law of the form $\sigma=K \varepsilon^{n}$ substituting into 3.21 gives: 


$$
\begin{aligned}
\frac{4}{3} K n e_{e}{ }^{n-1} & =\frac{2}{\sqrt{3}} K e^{n}{ }_{e} \\
\Rightarrow \quad \frac{2}{\sqrt{3}} n=e_{e} & =\frac{2}{\sqrt{3}} e_{22} \\
\therefore \quad e_{22} & =n
\end{aligned}
$$

Thus, theoretically, the same result is found as in the case of a round bar; the plane strain case should display instability at the same strain a round bar does. This point will be utilized later in chapter 5 where plane strain models will be investigated.

\subsubsection{Ductility:}

Ductility is a measure of the extent to which a material can be deformed plastically. It is commonly defined by two parameters, namely, $\%$ elongation and /or $\%$ reduction of area (ROA). Both are based upon measurements made after the specimen has fractured. These are given in the following forms (ASM (1992) and Hosford and Caddell (1993)):

$$
\% \text { Elongation }=\frac{100\left(L_{f}-L_{0}\right)}{L_{0}}
$$

Where $L_{0}$ and $L_{f}$ are the initial and final length of the gage section, before the test and at fracture respectively.

$$
\% \text { Reduction of area }(R O A)=\frac{100\left(A_{0}-A_{f}\right)}{A_{0}}
$$


Where $A_{0}$ and $A_{f}$ are the initial and final cross-sectional area of the specimen respectively. The measures above can be related by the following equation:

$$
\% \text { Elongation }=\frac{\% R O A}{(100-\% R O A)}
$$

Thus one can be calculated from the other, but this only holds as long as failure occurs without necking. After a neck has developed, the two are no longer related (ASM (1992)).

The disadvantage of using percent elongation as a measure is that it contains two parts, namely, the uniform elongation and the localized elongation. Uniform elongation occurs before necking while localized elongation occurs during necking, which makes it sensitive to the specimen shape. For round bars, if a small gauge length compared to the diameter is used to measure the elongation, then the necking will account for most of the total elongation. On the other hand, if a large gauge length compared to the diameter is used, the localized elongation as converted to percentage is very small. This problem is resolved for round bars by standardizing the specimen gauge length to diameter ratio to be $4: 1$. For a group of bars, which are of this same ratio of gauge length to diameter, the localization elongation will be the same fraction of the total elongation. The disadvantage of the ROA as a measure of ductility is the difficulty in measuring the cross sectional diameter at fracture (ASM (1992)).

\subsection{Plastic Behavior of Materials:}

In this section the plastic behavior of materials will be introduced with a focus on the behavior of metals. This serves as an introduction to numerical applications in the subsequent sections. 


\subsubsection{Yield Criteria:}

In the case of a simple tensile test it is fairly obvious what the criteria for yield is e.g.: $\sigma_{1}=Y$, where $Y$ is the yield stress in tension (or compression). However, things are somewhat more complicated for states of combined stress. A mathematical expression involving all the stresses is required. At first it is supposed that the yield stress could be represented by a function of nine independent components of the stress tensor to give:

$$
f\left(\sigma_{i j}\right)=0
$$

In the absence of body couples, the balance of momentum yields:

$$
\sigma_{i j}=\sigma_{j i}
$$

And thus equation 3.25 can be expressed as a function of only six independent components i.e.

$$
f\left(\sigma_{11}, \sigma_{22}, \sigma_{33}, \sigma_{12}, \sigma_{13}, \sigma_{23}\right)=0
$$

Since any state of stress can be expressed by the principal stresses and their directions, equation 3.27 can be written as:

$$
f\left(\sigma_{i}, \overline{n_{i}}\right)=0
$$

Where $\sigma_{i}(i=1,2,3)$ are the principal stresses and $n_{i}(i=1,2,3)$ are the unit vectors specifying the principal directions (cosine directions). Furthermore, if the material is isotropic, then there would not be any preferred directions and thus equation 3.28 can be expressed as:

$$
f\left(\sigma_{i}\right)=0
$$


Equation 3.29 can also be expressed in terms of stress invariants:

$$
f\left(I_{1}, I_{2}, I_{3}\right)=0
$$

Where $I_{1}=\sigma_{1}+\sigma_{2}+\sigma_{3}, I_{2}=\sigma_{1} \sigma_{2}+\sigma_{2} \sigma_{3}+\sigma_{3} \sigma_{1}$ and $I_{3}=\sigma_{1} \sigma_{2} \sigma_{3}$. Furthermore, it has been observed experimentally (Bridgman (1952)) that the hydrostatic pressure does not affect the yielding of metals i.e. the material behaves elastically under hydrostatic loading. Bridgman (1952) has shown that the volumetric deformation of a nonporous solid can be assumed to be elastic. This suggests that the yield criteria could be a function of the deviatoric stresses, which are the stresses minus the hydrostatic stress (pressure) as shown in equation 3.31 .

$$
\sigma_{i j}^{\prime}=\sigma_{i j}-\frac{1}{3} \sigma_{k k} \delta_{i j}
$$

Where

$$
\delta_{i j}= \begin{cases}0 & \mathrm{i} \neq \mathrm{j} \\ 1 & \mathrm{i}=\mathrm{j}\end{cases}
$$

Working out the deviatoric invariants, the following can be obtained:

$$
J_{1}=0, J_{2}=\frac{I^{2}-3 I_{2}}{3} \text { And } J_{3}=\frac{1}{27}\left(2 I^{3}{ }_{1}-9 I_{1} I_{2}+27 I_{3}\right)
$$

The most widely used yield criteria for metals was suggested by von Mises and assumes that the plastic yielding occurs only when the second invariant of the deviatoric stress tensor reaches a critical value, $c^{2}$, which is a material property and that $J_{3}$ was ignored as it is a high order function of the invariants (can be neglected). This criterion can be expressed as: 


$$
\begin{array}{ll}
J_{2}-c^{2}=0 & \text { For yielding or plastic deformation } \\
J_{2}<\mathrm{c}^{2} & \text { For elastic deformation }
\end{array}
$$

In terms of principal stress components, the yield criteria in its most commonly used shape, takes the form:

$$
\frac{1}{6}\left[\left(\sigma_{1}-\sigma_{2}\right)^{2}+\left(\sigma_{2}-\sigma_{3}\right)^{2}+\left(\sigma_{3}-\sigma_{1}\right)^{2}\right]=c^{2}
$$

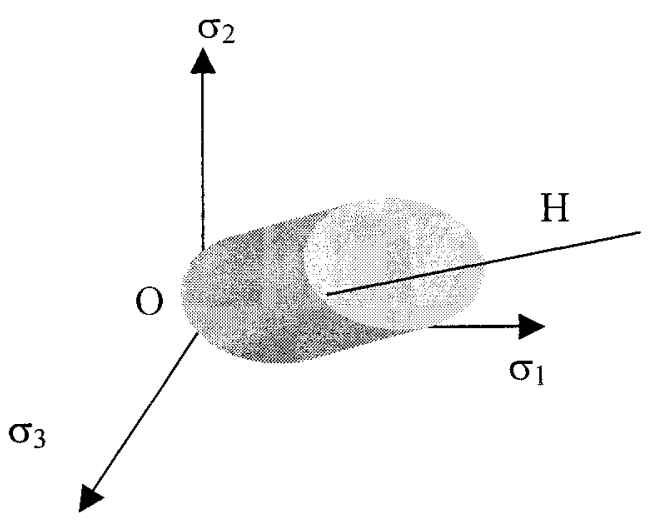

(a)

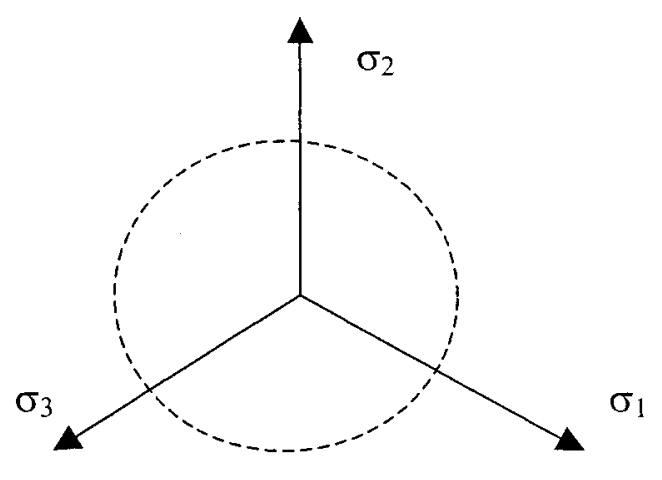

(b)

Fig. 3.4: (a) Von Mises flow stress surface in principal stress space (b) Synoptic plane.

The von Mises yield surface is a cylinder surface parallel to the hydrostatic stress axis $\left(\sigma_{1}=\sigma_{2}=\sigma_{3}=0\right)$ in the stress space as shown in Fig. $3.4 \mathrm{a}$. It is also convenient to view the model along the axis passing through $\mathrm{OH}$. This plane is called the synoptic plane. 


\subsubsection{Post-Yield Behavior: Plastic Stress-Strain Relations, Flow Rule:}

Incremental plastic strains are considered in the development of stress-strain relations since unlike the elastic deformation, the plastic deformation has a stress-path-dependent nature. To determine the total plastic strains, incremental strains through the history are accumulated. However, if a proportional stress path is followed (all stresses increase in the same ratio), the plastic strain will be independent of the stress history and will depend only on the final stress state. Although this simplifies the problem, it is not the general case but rather an exception, as most deformation processes are not proportional. In the next section the most common flow rules will be presented.

\subsubsection{Classical Development of Flow Rules:}

In the classical theory of plasticity, the material is assumed to be an ideal plastic material. This is assumed in the Levy-Mises flow rule, along with the assumption that the total strain increments are equal to the plastic strain increments; that is elastic strains are negligible. This serves also to assume with no reservation the constancy of volume, which can be expressed as:

$$
d \varepsilon^{p}{ }_{i i}=0
$$

And in deviatoric form of stress the deviatoric stresses for a test piece subjected to uniaxial tension are given in equation 3.31.

From 3.31 and 3.33 above, the following ratios were observed (Hill (1950)): 


$$
\frac{d \varepsilon^{p} 11}{\sigma_{11}^{\prime}}=\frac{d \varepsilon_{22}^{p}}{\sigma_{22}^{\prime}}=\frac{d \varepsilon^{p} 3}{\sigma_{33}^{\prime}}=\ldots \ldots \ldots . .=\frac{d \varepsilon_{13}{ }^{p}}{\sigma_{13}{ }^{\prime}}=d \lambda
$$

Which suggested the following form of flow rule:

$$
d \varepsilon^{p}{ }_{i j}=d \lambda \sigma^{\prime}{ }_{i j}
$$

Where $d \lambda$ is an increment that indicates the resistance to plastic flow. It is also referred to as the plastic modulus.

As mentioned earlier, the Levy-Mises flow rule above neglects the elastic strains, which in certain circumstances may be significant. The Prandtl-Reuss equation, which will be shown below, allows for elastic strains and are based on the observation that the total strain increment is formed by adding the elastic and plastic strain increments:

$$
d \varepsilon^{\prime}{ }_{i j}=d \varepsilon^{e}{ }_{i j}+d \varepsilon^{p}{ }_{i j}
$$

Prandtl-Reuss adopted the Levy-Mises equation and added to it the elastic strain to make up the total strain increment, and thus the same derivation could be used to develop their flow rule. The plastic strain increment can be related to the stress states in the tensor form in the same way as it was shown in equation 3.35. Or more usefully in terms of equivalent stress and equivalent strain increment by:

$$
d \varepsilon_{i j}^{p}=\frac{3}{2} \frac{d \varepsilon^{p_{e}}}{\sigma_{e}} \sigma_{i j}^{\prime}
$$




\subsubsection{Generalized Plastic Stress-Strain Relations:}

In the previous section, the development of the stress-strain relations was given for special cases of simple ideal plastic material, and thus the development of the equations was also simple. A generalized approach to establish the constitutive equations is accomplished by introducing the plastic potential function. Considering the role of the strain energy function in the elastic counterpart, it is assumed that it is possible to obtain the plastic strain increment, $d \varepsilon^{p}{ }_{i j}$, from the plastic potential, $Q\left(\sigma_{i j}\right)$. The increment of the plastic strain can be expressed as follows:

$$
d \varepsilon^{p} i j=\lambda \frac{\partial Q}{\partial \sigma_{i j}}
$$

Where $Q$ is the plastic potential function. This relation is also referred to as the normality rule. For some materials the plastic potential function, $Q$, and the yield function $f$, can be assumed the same. Such materials are considered to follow the associative flow rule.

The yield function can be used to obtain the stress-strain relationship using the associative flow rule. Often, materials harden after yielding which makes it easier to establish the stress-strain relations using the generalized plastic potential method. The advantages of the associative flow rule is that it establishes the normality rule for the plastic strain increment tensor, as equation 3.38 implies that the plastic strain increment vector $d \varepsilon^{\prime \prime}{ }_{i j}$ is directed along the normal to the surface of $Q\left(\sigma_{i j}\right)$. It also establishes the convexity criteria for the yield surface and positive definitions of plastic energy dissipation (Davis and Selvadurai (2002)). 


\subsubsection{Hardening Rules:}

The process of work hardening is the increase in the yield limit as a result of plastic deformation of the material. Hardening processes are important in the description of yield and failure in the materials. It considerably affects the plastic deformation of the material depending on the rate of hardening the material possesses. It is obvious that the yield functions, which mark the boundaries of the elastic domain, must depend not only on the current stress state but also the loading history. The history is introduced through hardening variables or parameters that keep record of plastic deformation the material has experienced. The simplest hardening rule is isotropic hardening in which the yield surface simply expands in all directions in a similar manner. Other hardening types are also used for metals, like the kinematic hardening rules in which the yield surface translates in the stress space. Combined hardening rules are those, which constitute expansion and translation of the yield surface. Schematic description of the three hardening rules is shown in Fig. 3.5. 


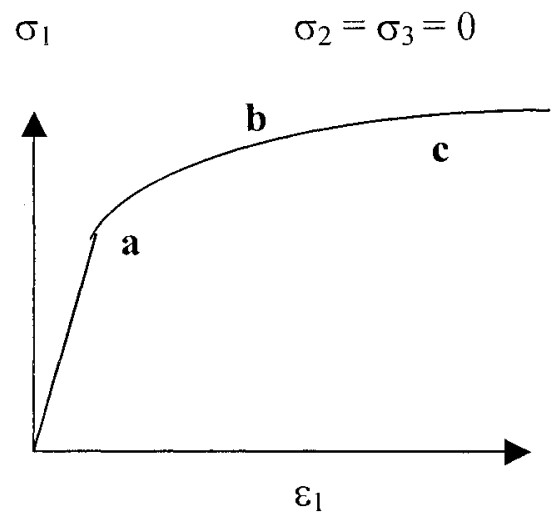

(a)

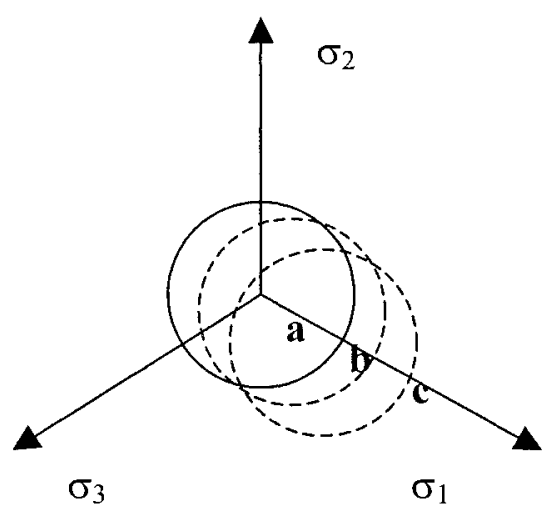

(c)

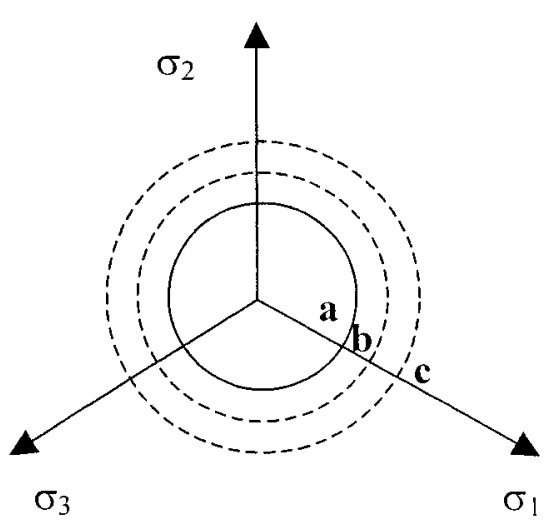

(b)

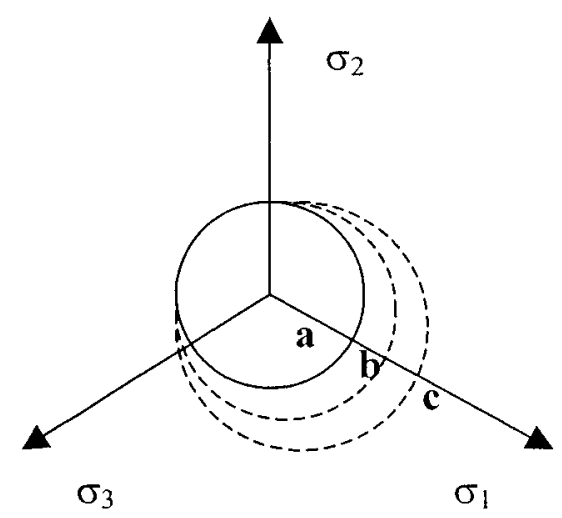

(d)

Fig. 3.5: (a) Stress-strain curve in uniaxial tension and its effect on yield loading for (b) Isotropic hardening (c) Kinematic hardening and (d) Combined hardening. 


\section{CHAPTER}

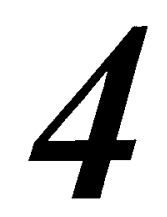

\section{EXPERIMENTAL AND METALLURGICAL CONSIDERATIONS}

In this Chapter the experimental objectives coupled with metallurgical concepts and considerations will be discussed. A background on some aspects of metallurgy, which is essential to this work, will be presented. Finally, experimental procedures, material used in this research and the experimental results will be presented. The results of this Chapter and the next two Chapters, where micro mechanical modeling is used to characterize the DP-steels, will be compared in Chapter 7 as validation of the modeling technique used in this research work. In the modeling procedure, as will be shown later, none of the experimental results on dual phase steel will be used to model the material micro mechanically, and thus the comparison between the experimental work which will be presented here and the modeling results in the next Chapters is presented solely as experimental validation of this research work. 


\subsection{Metallography:}

The concept of micro mechanical modeling relies on the prediction of material response based on micro structural properties of the material. In order to visualize the microstructural properties of the material, it is essential to use proper metallographical techniques, which facilitate the determination of the main characteristics or features of the material to be modeled. In addition, knowing the details of the microstructure is important in order to understand the relationship between processing parameters and the behavior of materials.

Three important metallographical techniques are, namely, optical microscopy, scanning electron microscopy (SEM) and transmission electron microscopy (TEM). The optical microscopy and the SEM are used to characterize material structures by revealing grain boundaries, phase boundaries and inclusion distribution. The TEM, on the other hand, which has very high magnification capability, is used to examine dislocation structures, small defects in the metal, small second phase particles and details which are not possible to visualize using the two former techniques due to magnification constraints.

The choice of the magnification technique mentioned above relies on the microstructural information sought, as each will serve as the best tool for certain levels of material microstructural characterization. The first step in the examination of any microstructure is using optical microscopy, which has the least magnification capability, but the most effective way to see grains, second phase particles, grain boundary and second phasematrix interface. For characterization of the DP-steels, where it is necessary to examine the constituents, volume fraction of each and the distribution of the phases; optical microscopy is the proper and the customary tool. In the case where phase structure is sought e.g. martensite structure which is too fine; or when the nucleation and growth of voids is investigated, the higher magnification of the SEM is needed. 
The majority of microstructural investigation by optical microscopy is carried out with vertical illumination (also called bright field). This type of light microscopy uses reflected light with no need for additional manipulation of the light beam. Basically, the areas that lie normal to the incident light reflect back with the greatest intensity making it appear brightest. The oblique illumination (also called dark field) uses the opposite illumination to the bright field where dark regions appear bright and bright regions appear dark. This is achieved if the direction of the incident light is changed from being normal to the incident surface. This technique is useful for checking the quality of polished surfaces since scratches clearly show as bright lines. It is also useful in viewing the grain structure and crystal defects, which is ideal for investigating martensite structure (ASM (1985)). Polarized light microscopy and phase contrast illumination are also two other techniques where the first is useful for differentiating isotropic and anisotropic components of a structure and the second one produces images with emphasized topographic details (Chan and Haasen (1996)).

\subsubsection{Material Preparation for Microstructural Visualization:}

There are several stages of material preparation required for any visualization. There are features that can be visualized without prior surface preparation, such as when one needs to look at the fracture surface of the material, but most of the other investigations require surface preparation, which is an important stage of metallography.

\section{A) Sectioning:}

The removal of a representative part from a layer of a sample is called sectioning. Sectioning is the first of five operations in the preparation of metallographical specimens. The starting point of metallography is sectioning; which is the most important step in specimen preparation for microscopic examination and analysis. Incorrect specimen preparation may change the true microstructure and lead to errors in the calculations. 
Thus operations that change the true material microstructure should be avoided, which include any operation, which introduces high heat flux to the material (ASM (1985)).

There are many sectioning methods reported in the literature, namely, fracturing, shearing, sawing, abrasive cutting and wire sawing. Each method has its advantages and disadvantages in terms of damage it causes to the sample and cost of employing it. The oldest and the most economical method is the sawing method, which can be performed using a handsaw or an oscillating power hacksaw. Hand held hacksaws do not generate enough frictional heat to alter the microstructure, but power hacksaws can irreparably damage a material. In this work handsaws were mainly used to cut the heat-treated specimens. The speed is low and interrupted, which introduces a very small depth of deformation (ASM (1985) and ASM (2000)).

\section{B) Mounting:}

Although proper shaped samples may not require mounting, small and oddly shaped specimens should be mounted for easy handling during preparation and examination. Mounting the specimen will also eliminate the sharp corners in the samples, which avoids damage to material used in the subsequent material preparation and provides safety to the metallographer. In addition, proper selection of the mounting method and material would preserve the sample, make it more easily stored, and provides a means of using these standard sizes of mountings to grind and polish sets of samples in one operation using automatic grinding and polishing facilities.

There are several mounting techniques available, but certain metallographical techniques such as SEM require the mounting material to be electrically conductive. An essential requirement in any mounting material is that mounting should not cause defects in the sample. The mounting material and the specimen should have similar grinding and polishing features (ASM (2000)). 
Mounting in this work was done using plastic mounting materials, which is in a powder form, such as thermosetting resin. Thermosetting resins require heat and pressure during molding, but can be ejected from the mold at the molding temperature. This method requires certain standard pressure, temperature and time, which are specific for each material. Other means and techniques are also available which are referred to as special techniques for specimens, which require special methods (ASM (1985))

\section{C) Mechanical Grinding, Abrasion and Polishing:}

Surface preparation is required to investigate the structure of the materials. All the techniques involve the reflection of some form of radiation or light from the section surface of interest. Crystal structure or composition variation can be detected by the change of the reflected radiation or light. The surface of interest is usually treated physically and /or chemically in order to alter its attributes to change the way the various constituents of the material reflect light. Another technique involves striking a surface with a beam of electrons in a vacuum. The structures are displayed by how electrons are reflected back. An important example of such technique is SEM. The TEM requires preparation of two parallel plates in close vicinity; the radiation used is transmitted through this thin slice (ASM (2000), Williams, Cater (1996) and Goldstein et al. (1981)).

\section{1) Grinding and Abrasion:}

Employing an array of fixed abrasive particles with projecting points that act as cutting tools is called grinding. Grinding employs high surface speeds with the possibility that significant heating of the surface layer of the specimen may occur. Abrasion, on the other hand, refers to processes that employ low surface speeds and continuous liquid coolant; and significant heating of the specimen surface cannot occur (Modin and Modin (1973) and ASM (1985)). In this work, the material preparation process used to make a very 
smooth surface is abrasion, at low speeds with continuous use of cooling water to avoid any change in the microstructure.

\section{2) Polishing:}

The objective of this stage of surface preparation process is to produce a bright mirrorlike surface commonly referred to as a polished surface. A polishing process is carried out in the same way abrasion and grinding are carried out, except that the abrasive particles that are used to polish the surface are not fixed as in the case in abrasion and grinding; but is in a liquid form in the fibers of a cloth.

Typical surface preparation procedures involve employing a sequence of grinding of increasing fineness, then a sequence of abrasion of increasing fineness followed by a sequence of polishing processes of increasing fineness to develop the surface finish required. The fineness refers to the use of finer grades of abrasive material to produce finer grooves in the surface. In addition, each step of finer abrasion is applied perpendicular to the previous coarser step to enhance the effect of finer abrasive. Most mechanical polishing procedures are similar to those of abrasion except that smaller forces are applied to individual abrasive particles by the fibers of the cloth that support them (ASM (1985), ASM (2000) and Modin and Modin (1973)). Polishing in this work is carried out using $3 \mu \mathrm{m}$ and $1 \mu \mathrm{m}$ diamond paste in sequence on a rotating disc at approximately 150 RPM.

\section{D) Etching:}

Etching is the final step in preparing the material for investigation by different techniques of visualizing the microstructure. Generally, it is based on the concept that when a crystal is dissolved in a solvent, the dissolution rate in the different crystal directions, grain boundaries, grooves, grain height differences, or orientation difference between grains and phases is not the same. This phenomenon is utilized to visualize the metal grains in a 
polished metal surface. During etching, the crystal grains are etched at different rates dependent on the grain orientation and height, which develops a surface of different slopes. If this surface is inspected using a bright field illumination microscope, the grains appear light, surrounded by darker grain boundaries. "The grain boundaries thus take the form of more or less inclined surfaces which reflect less light into the objective than do horizontal surfaces, the amount decreasing the steeper the angle of slope. In general, the grain boundaries are not attacked preferentially and do not form troughs (Modin and Modin (1973)." (ASM (1985)).

The same idea is used for steels with different phases. If there are several phases present, they are etched at different rates. In Carbon steel, for example, the etching solutions generally used are the ones that do not attack the carbide particles so that after etching, the carbide particles appear above the ferritic surface (Modin and Modin (1973)). The same approach will work for DP-steels where different phases coexist; the phases react differently to the etchant and thus can be visualized as different entities in the microstructure.

\subsubsection{Measurements and Image Analysis:}

Constraints in image analysis, as a whole, prevail in that contrast differences between the constituents have to be visible. In addition, they are based on measurements done on two dimensional surfaces while seeking quantitative analysis of the characteristics of the material on the real three dimensional basis. These problems are tackled by introducing the improved metallography techniques and the concept of stereology, which will be explained later in this section.

"Stereology is the body of measurements that describe relationships between measurements made on the two-dimensional plane of polish and the characteristics of the three-dimensional microstructural features sampled (ASM (2000))". Quantitative metallurgical measurements pertinent to this work will be presented below. 


\section{A) Volume Fraction and Area Fraction:}

The determination of the amount of the second phase or constituent in a two-phase alloy is an important measurement, as it is well known that the amount of the second phase can have significant influence on its properties and behavior. Random two dimensional section planes may be utilized to obtain the volume fraction. Manual methods have been used for many years and are still being used in the present. One method involves measurement of area; another, the measurement of lineal intercept and a third method is called counting points. The three main methods above are called areal analysis, lineal analysis and point counting respectively. A French geologist, Delesse, (see Underwood (1970), Dehoff and Rhines (1968) and ASM (2000)) was the first to indicate the equivalence of the volume fraction to the area fraction. It has also been shown that the volume fraction is also equal to lineal fraction and point fraction. It is worth noting that these equalities are exact as no simplifying assumptions of features in the microstructure are required. However, the measurements must be made randomly or with a specific statistical uniformity. In addition, the test section examined must be representative of the entire sample. The equality of the volumetric, areal, lineal and point count is generally expressed in the following form:

$$
V_{V}=A_{A}=L_{L}=P_{P}
$$

Simple derivations are presented in Underwood (1970). Consequently, several procedures are used to estimate the volume fraction of the constituents in a two-phase material each based on the above methods. The basic idea in areal method is measuring the area, weight or square grids in a microstructural image, which lies in the phase of interest and dividing that by the total area, weight or square grids. Lineal analysis and point count could also be used to get the volume fraction. The sum of the lengths of line segments within a phase of interest is divided by the total length in the first method and 
the number of points lying in the phase of interest is divided by the total number of points in the grid in the second method.

All the above methods give accurate results. However, the point count method is considered the most efficient method, which yields the best precision with minimal effort and will be used in this work. The point count method is described in detail in ASTM E 562 and is widely used to estimate the volume fraction of the second phase. In the methods above, the procedure should be repeated on a number of fields selected in a statistically uniform way, which would represent the entire polish surface without bias. Guidelines on procedures are given in ASTM E 562.

\section{B) Grain Size Measurement:}

There are two methods of determining the grain size in a polished sample, namely, the lineal intercept method (also referred to as Heyn method) and the circular intercept method. The first method is based on estimating the average grain size by counting the number of grains intercepted by one or more straight lines sufficiently long. The grain size is then determined by dividing the total length of the line by the number of intercepts. In the latter method, use of circular test lines is made rather than straight test lines. The details of the procedures are given in ASTM E 112-96. Nonequiaxed grain measurement is made in three principal planes and the average of the three is determined. For twophase grain structures, the grain size of a particular phase requires the determination of the volume fraction of the constituents. For example, by point counting, the minor or second phase is counted and the volume fraction of the matrix or major phase is determined by the difference. Next, using lineal or circular measurement, the mean lineal intercept for the phase of interest is determined from:

$$
l_{\alpha}=\frac{\left(V_{V}\right)(L / M)}{N_{\alpha}}
$$

Where $L$ is the length of the line and $M$ is the magnification factor, $V_{V}$ is the volume fraction and $N_{\alpha}$ is the number of grain intercepted by the test line. This measurement 
could have also been done by counting the intercepts for the phase of interest only and divide the total length of line used deducted from it the length, which intercepts the other phase. It is worth mentioning that it is well recognized that the field-to-field variability in volume fraction has a larger influence on the precession of the volume fraction and grain size estimates than the error in rating a specific field, regardless of the procedure used $(\operatorname{ASM}(2000))$.

In the above sections, manual measurements of the volume fraction and grain size have been presented which require considerable effort to obtain acceptable measurement accuracy especially when the volume fraction of the second phase is small. Image analyzers are widely used to overcome this problem. A computer connected to a microscope performs measurements in image analyzers. These can be direct measurements such as size, area, longest dimensions or indirect measurements, which can be derived from these such as shape and circularity. By using the image analyzer enough field measurements can be made in a relatively short time. There are two image analyzerbased standards developed, ASTM E 1122 and ASTM E 1245. The latter is the stereological approach, which is of interest in this work. The image analyzer devices rely primarily on the gray level of the image on the monitor to detect the desired feature. Any image is composed of small, usually square, picture elements called pixels. The gray level, or intensity, of each pixel relates to the light photons striking the detector.

Area and Volume Fraction: Area fraction is an easy measurement done using an image analyzer. In this measurement, the computer scans the number of pixels contained in the feature of interest and divides that by the total number of pixels in the image. This operation is easily performed by visualizing the gray level histogram to monitor the extent to which the feature of interest should be accounted for. From a stereological standpoint, the area fraction is equivalent to the volume fraction.

Grain size: Intercept measurements can also be achieved easily using an image analyzer. After adjusting the length scale of measurement to that of the micrograph used, parallel horizontal and vertical lines can be drawn in one field and interceptions can be counted 
and divided by the total length of the lines. The grain size is then calculated based on measurement on different fields and the mean lineal intercept is usually reported (ASM (2000)).

\subsection{Materials and Experimental Procedures:}

In this section, the experimental investigation of the behavior of the DP-steels, consisting of martensite in a matrix of ferrite will be presented. Micro alloyed low carbon steel is used to produce several $V_{m}$ and tested mechanically to investigate their mechanical properties.

\subsubsection{Materials:}

The material used in this work was a low carbon steel with chemical composition in wt.\% determined using vacuum emission spectroscopy as shown in table 4.1 below:

\begin{tabular}{|c|c|c|c|c|c|c|c|c|c|c|}
\hline Alloy & $\mathrm{C}$ & $\mathrm{Mn}$ & $\mathrm{Si}$ & $\mathrm{Cr}$ & $\mathrm{Ni}$ & $\mathrm{S}$ & $\mathrm{P}$ & $\mathrm{Mo}$ & $\mathrm{N}$ & $\mathrm{Cu}$ \\
\hline \%wt & 0.09 & 1.5 & 0.9 & 0.06 & 0.08 & 0.005 & 0.01 & 0.004 & 0.005 & 0.04 \\
\hline
\end{tabular}

Table 4.1: Steel chemical composition in weight percent.

This material was received in the form of wire rod of diameter of $10 \mathrm{~mm}$, with a microstructure consisting of martensite and equiaxed ferrite only. The received material was heat treated to obtain DP-steels of different $V_{m}$ by water quenching from different intercritical annealing temperatures to room temperature. The heat treatment of the samples was done in a furnace in which the samples were placed vertically in crucibles to 
ensure uniform heat treatment through the diameter and the length of the samples. Preliminary trials were carried out first in order to get the desired microstructure of the

\begin{tabular}{||l|l|l|}
\hline $\mathrm{V}_{\mathrm{M}} \%$ & ANNEALING TEMPERATURE $\left({ }^{\circ} \mathrm{C}\right)$ & TIME \\
\hline $14 \%$ & $750^{\circ} \mathrm{C}$ & 15 minutes \\
\hline $19.7 \%$ & As received & As received \\
\hline $34 \%$ & $825{ }^{\circ} \mathrm{C}$ & 10 minutes \\
\hline Ferrite & $1000^{\circ} \mathrm{C}$ & 21 hours \\
\hline
\end{tabular}

Table 4.2: Heat treatment details and volume fraction of martensite produced.

material on small discs from the same material. These trial heat treatments were checked by a light microscope and then the material cylinders were used to obtain the tensile specimens. The heat treatment details are presented in table 4.2:

The ferrite phase was also produced as shown in table 4.2 , which is required to model the material micro mechanically as will be shown in the next Chapter. The heat treatment to produce ferrite was carried out by decarburizing the steel for 21 hours at approximately $1000^{\circ} \mathrm{C}$ followed by furnace cooling, to produce a carbon free steel. This process of heat treatment produced $6 \%$ pearlite in addition to ferrite, which is a good approximation of the ferrite phase as the pearlite phase is not much stronger than the ferrite phase and due to it's low percentage, would not have significant influence on the material behavior. To remove decarburized layers on the tensile samples, they were machined to $6 \mathrm{~mm}$ diameter, which also ensures a uniform heat treatment through the diameter. 


\subsubsection{Mechanical Testing:}

After heat treating the cylindrical bars according to table 4.2, tensile specimens with threaded ends were made from them and tested in uniaxial tension on a Material Testing Systems (MTS) machine which is equipped with an automatic controller using the displacement control mode at a quasi static rate of $0.05 \mathrm{~mm} / \mathrm{sec}$. The dimensions of the tensile specimen are shown in Fig. 4.1.

A one-inch extensometer was used to measure the strain in the specimens. Photographs of the tensile specimen after fracture are shown in Fig. 4.2. The ratio of the gauge length to the diameter is taken as nearly $4: 1$. The tensile tests were performed at room temperature and the resulting load-strain data obtained directly from the MTS machine was converted to engineering stress-strain curves.

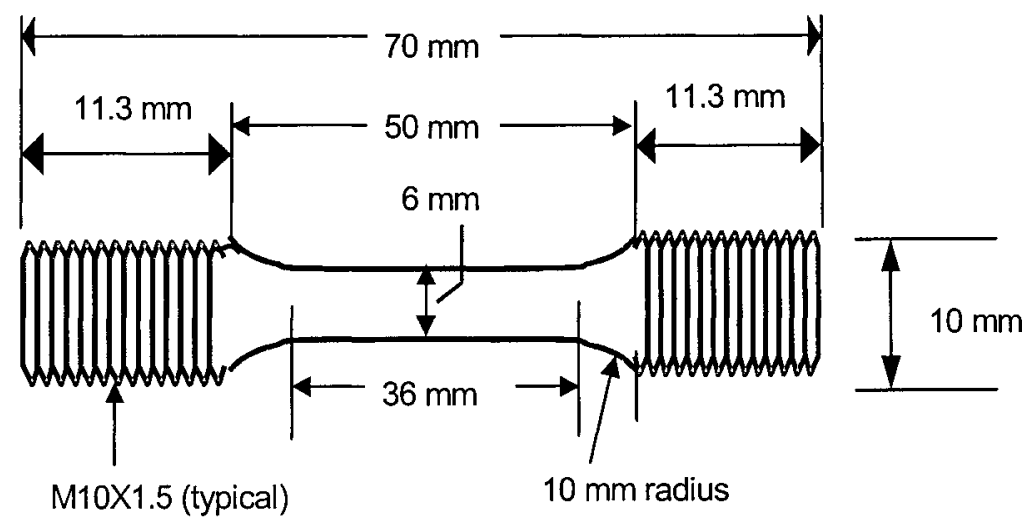

Fig. 4.1: Tensile specimen dimensions (not to scale)

The one-inch $(25.4 \mathrm{~mm})$ extensometer is relatively accurate but it is limited in measuring strain or elongation. It can measure up to approximately $17 \%$ nominal strain as shown in Fig. 4.3. The MTS displacement reading, which extends to fracture as shown in Fig. 4.4, is not accurate especially at the beginning of the curve or at small strains (ASM (1992)). In addition, the original length, which is used to calculate the \% elongation, is somewhat a debatable figure that could be extended to include the part between the ends of the 
threaded parts (shoulder) to the beginning of the shank of the tensile specimen. These two limitations in measurements were resolved by combining measurements made by the extensometer for as large as it can read with the measurements of the crosshead reading (MTS reading) to include strains to fracture. In doing this, the crosshead MTS measurement, which is not accurate, is corrected based on manually measured final elongation
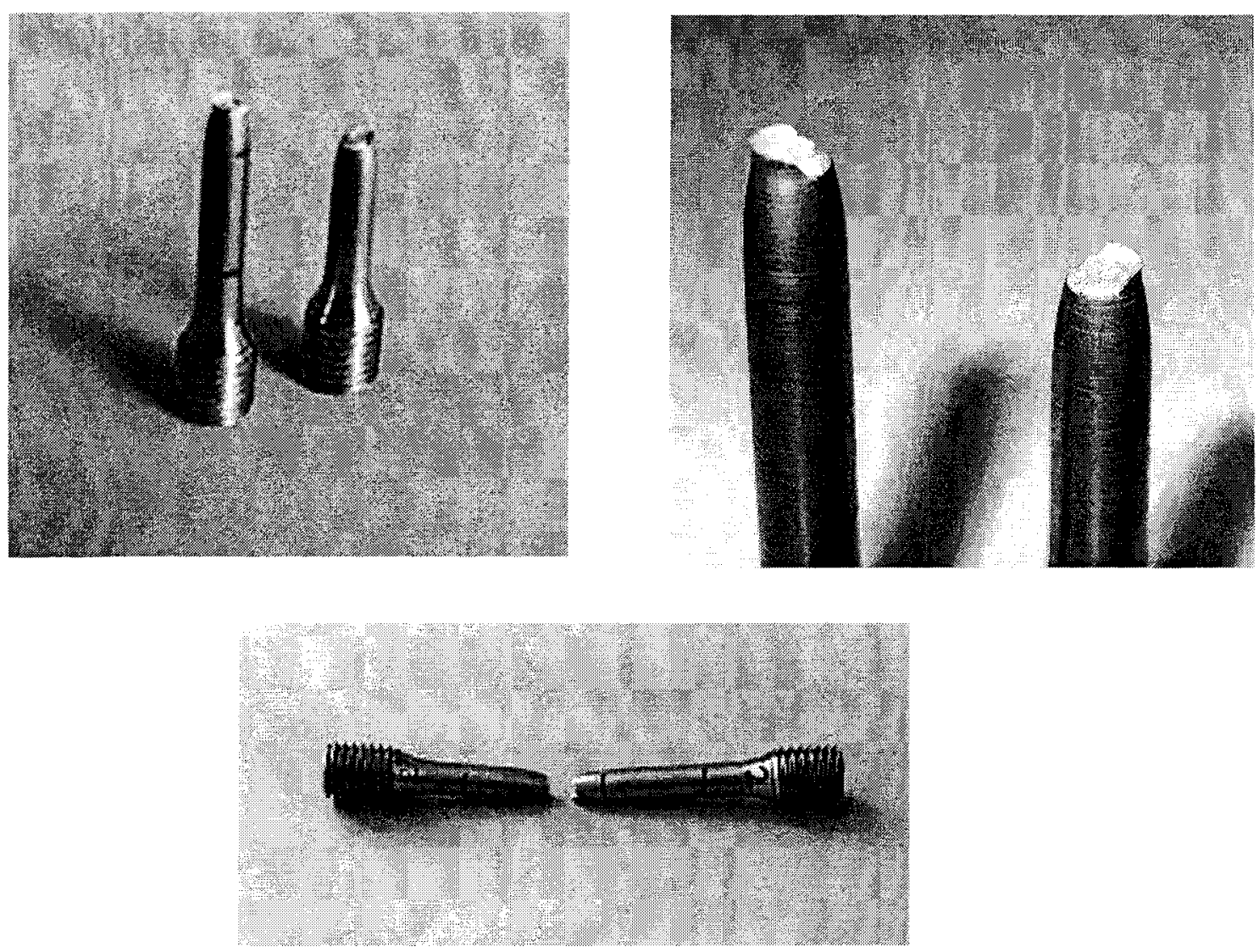

Fig. 4.2: Typical tensile specimen after fracture.

The final elongation is measured on the specimens by marking 1 inch distanced thin lines on the specimens before testing and after the test, fractured parts are put together and the distance between the marks is measured to get the final elongation. 


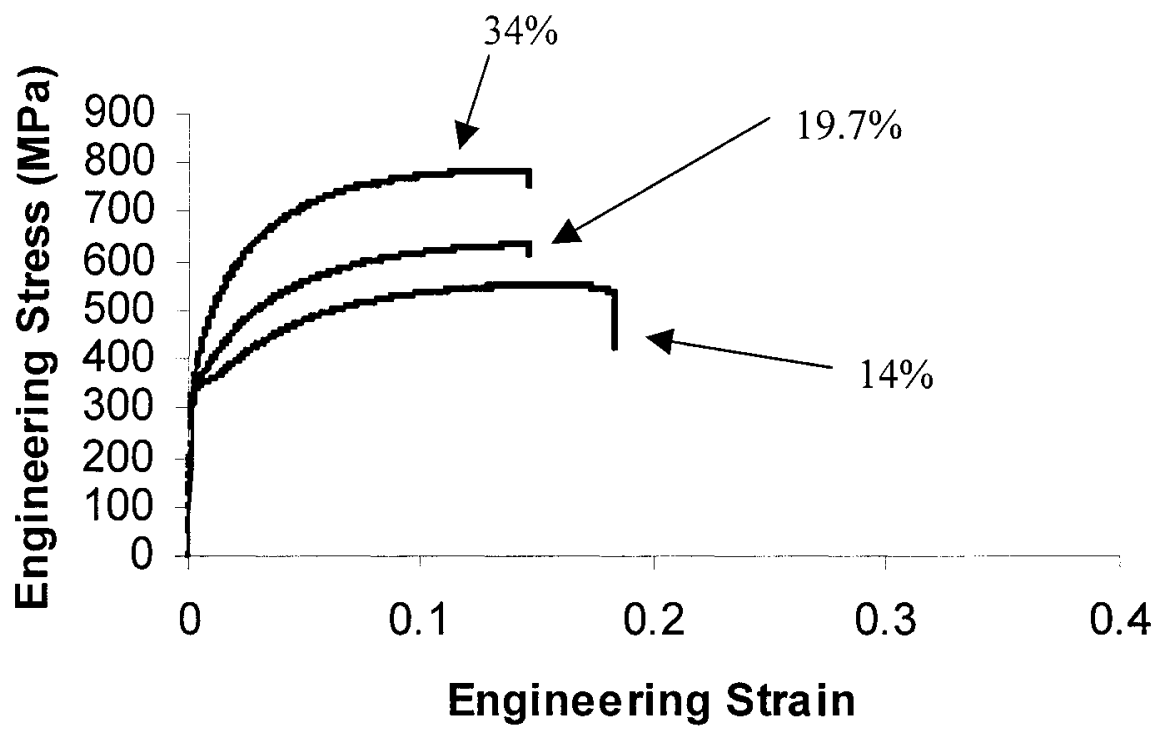

Fig. 4.3: Extensometer measurement of the engineering stress strain curve for $V_{m}$ of $14 \%, 19.7 \%$ and $34 \%$.

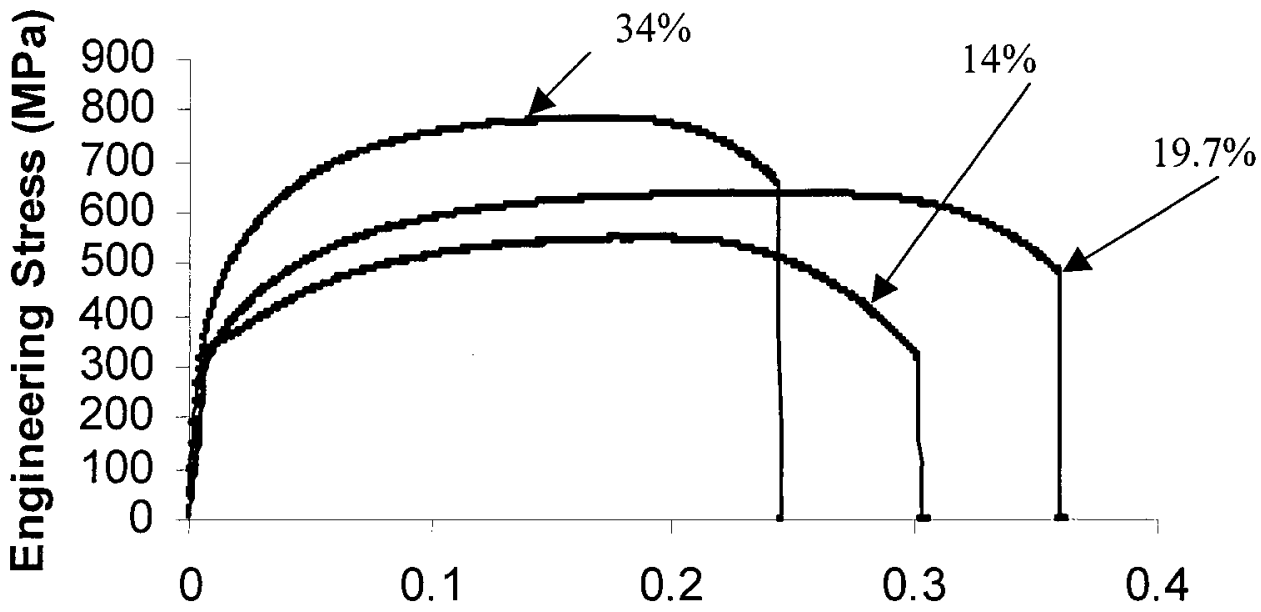

Engineering Strain

Fig. 4.4: MTS crosshead measurement of the engineering stress strain curve after correcting the final elongation to match the manually measured one for $V_{m}$ of $14 \%$, $19.7 \%$ and $34 \%$. 
The crosshead MTS reading is fitted to match the extensometer reading and the final elongation measured manually, which are two well-founded measurements. This is done in a trial and error method by multiplying the crosshead reading by a factor which makes the crosshead reading match both the measured final elongation and the stress-strain trend of the extensometer reading for the low strain range at the same time. These measurements are repeated for each specimen tested. The best fit is found as shown in Fig. 4.5 from which a combined curve is obtained as will be shown in the next Chapters where experimental results will be compared to the micro mechanical model predictions.

The engineering stress-strain curves for three $V_{m}$ produced, namely $14 \%, 19.7 \%$ and $34 \%$ are shown below in Fig. 4.5. As can be seen from the figure, as the $V_{m}$ increases, the strength and ductility (\% elongation) increases as seen for $V_{m}=14 \%$ and $V_{m}=19.7 \%$. Increasing the $V_{m}$ to $34 \%$ still raises the strength of the aggregate but the ductility deteriorates.

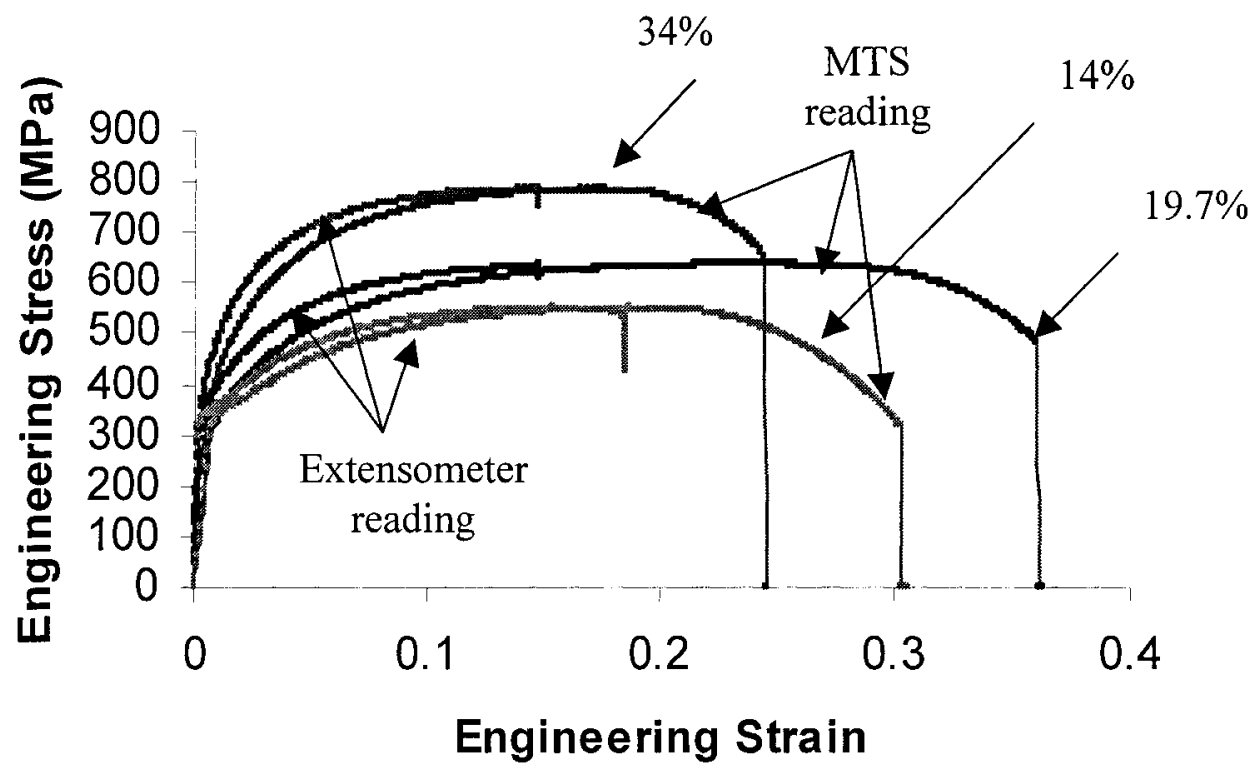

Fig. 4.5: Representative engineering stress-strain curves for $V_{m}$ of $14 \%, 19.7 \%$ and $34 \%$. 
The single ferrite phase produced as shown in table 4.2 was also tested and a representative stress-strain curve is shown in Fig. 4.6. This curve will be used in the micro model as will be discussed in detail in the next Chapter. Three to four specimens for each $V_{m}$ were tested and the repeatability of all the curves were excellent and almost all the necking occurred between the extensometer pins with exceptions. The mechanical properties for all the tested specimens are shown in detail in table 4.3.

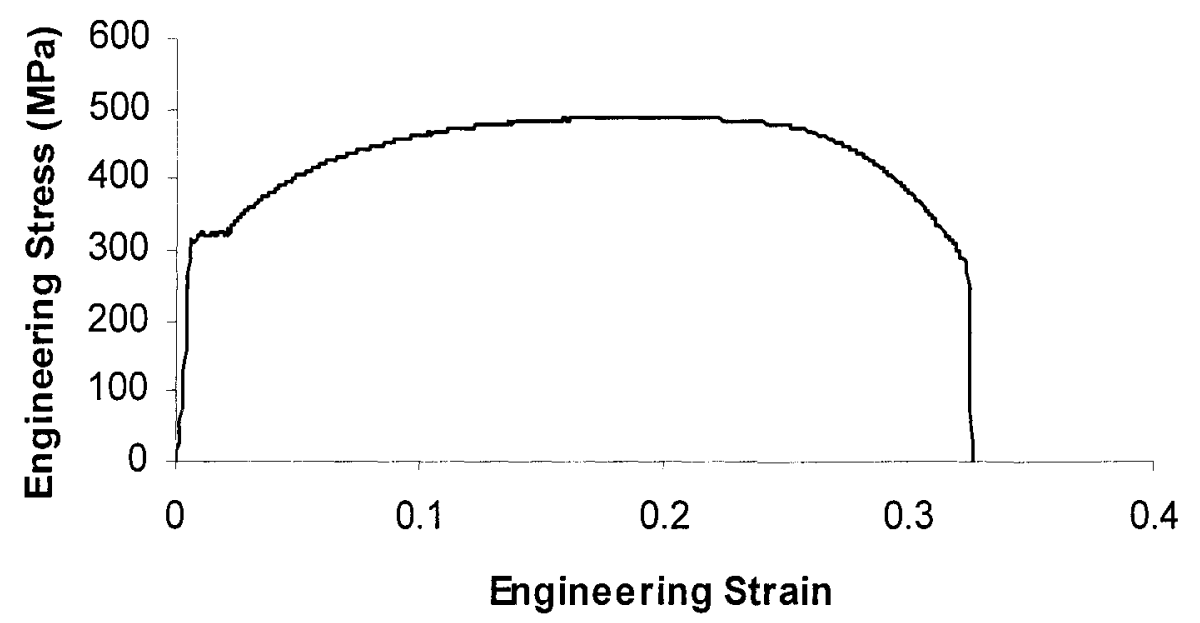

Fig. 4.6: Single ferrite stress-strain curve, sample annealed at $1000^{\circ} \mathrm{C}$ for 21 hours.

\subsubsection{Microstructure:}

Fig. 4.7 shows representative optical micrographs of DP-steels made or produced in this work. The specimens examined for microstructure were cut from the threaded portion of the specimen as this region undergoes minimum deformation. The samples were mounted in the Struers Labopress-3 mounting press with a thermosetting phenolic powder 


\begin{tabular}{|c|c|c|c|c|c|c|}
\hline $\begin{array}{l}\text { Volume } \\
\text { fraction } \\
\%\end{array}$ & $\begin{array}{c}\text { Elongation } \\
\text { to fracture } \\
\%\end{array}$ & $\begin{array}{l}\text { R.O.A } \\
\%\end{array}$ & $\begin{array}{c}\text { UTS } \\
\text { (MPa) }\end{array}$ & $\begin{array}{c}\text { Uniform } \\
\text { strain } \\
\text { (Max.) } \\
\% \\
\end{array}$ & $\begin{array}{c}\text { Final } \\
\text { elongation } \\
(\mathrm{mm})\end{array}$ & $\begin{array}{c}\text { Note \& Heat } \\
\text { treatment }\end{array}$ \\
\hline $\begin{array}{l}V m=14 \\
\pm 0.56 \%\end{array}$ & $\begin{array}{c}36.81 \\
35.24 \\
35.55 \\
(35.08) ? \\
35.43 \# \\
35.51 \\
32.60\end{array}$ & $\begin{array}{l}68.10 \\
69.14 \\
67.06 \\
69.49 \\
70.42 \\
71.65 \\
67.21\end{array}$ & $\begin{array}{l}576.00 \\
572.40 \\
582.70 \\
576.80 \\
550.00 \\
558.57 \\
549.37\end{array}$ & $\begin{array}{l}15.20 \\
15.25 \\
15.50 \\
11.20 \\
17.10 \\
15.07 \\
15.55\end{array}$ & $\begin{array}{l}34.75 \\
34.35 \\
34.43 \\
34.31 \\
34.40 \\
34.42 \\
33.68\end{array}$ & $\begin{array}{l}\text { Cup cone failure } \\
750^{\circ} \mathrm{C} \text { for } 15 \\
\text { minutes }\end{array}$ \\
\hline $\begin{array}{l}V m= \\
19.7 \pm \\
0.86 \%\end{array}$ & $\begin{array}{l}36.08 \\
35.75 \\
33.46\end{array}$ & $\begin{array}{l}55.89 \\
54.21 \\
53.94\end{array}$ & $\begin{array}{l}635.18 \\
636.04 \\
640.29\end{array}$ & $\begin{array}{l}14.70 \\
15.40 \\
14.80\end{array}$ & $\begin{array}{l}34.55 \\
34.48 \\
33.90\end{array}$ & $\begin{array}{c}\text { Cup cone failure } \\
\text { As received }\end{array}$ \\
\hline $\begin{array}{l}V m=34 \\
\pm 1.95 \%\end{array}$ & $\begin{array}{l}(19.76) ? \\
23.42 \\
24.41 \\
22.00\end{array}$ & $\begin{array}{l}42.94 \\
48.97 \\
49.43 \\
44.29\end{array}$ & $\begin{array}{l}801.43 \\
791.64 \\
783.86 \\
792.91\end{array}$ & $\begin{array}{l}12.44 \\
10.56 \\
15.10 \\
10.66\end{array}$ & $\begin{array}{l}30.42 \\
31.35 \\
31.60 \\
30.99\end{array}$ & $\begin{array}{l}\text { Cup cone failure } \\
825^{\circ} \mathrm{C} \text { for } 10 \\
\text { minutes }\end{array}$ \\
\hline $\begin{array}{l}\text { Ferrite + } \\
6 \% \mathrm{Vp}^{*}\end{array}$ & $\begin{array}{l}34.49 \\
38.20 \\
37.10\end{array}$ & & $\begin{array}{l}488.40 \\
492.30 \\
492.90\end{array}$ & $\begin{array}{l}20.10 \\
23.70 \\
22.10\end{array}$ & $\begin{array}{l}34.16 \\
35.10 \\
34.82\end{array}$ & $\begin{array}{l}\text { Cup cone failure } \\
21 \mathrm{hrs} \text { at } 1000^{\circ} \mathrm{C} \\
\& \text { furnace cooling }\end{array}$ \\
\hline
\end{tabular}

* Vp: volume fraction of pearlite

\# Different shape of failure than the others

? approximated as measurement lines were wide after failure

R.O.A: Reduction of area.

Table 4.3: Mechanical properties of the DP-steels tested. 
resin (Bakelite) using a force of $20 \mathrm{kN}$, heating at approximately $180^{\circ} \mathrm{C}$ for 6 minutes and cooling for 3 minutes. The samples were ground using grit sizes of $60,120,240,320$, 400, 600 and 1200 grit silicon carbide papers in sequence, polished using $3 \mu \mathrm{m}$, and $1 \mu \mathrm{m}$ diamond paste in sequence on a rotating disc at about 150 RPM and finally etched using a $2 \%$ Nital solution.

The volume fractions and grain sizes were determined using a Clemex vision version 3.5 image analyzer equipped with a Nikon model Epiphot 200 optical microscope. The volume fraction measurements were based on area percent, which is the ratio of the total detected phase area to the image frame area using the point count method as explained in section 4.1.2. In order to obtain a good representation of the microstructure, a magnification of 500 times was chosen and 16 fields in each of the four coordinate directions and in the diagonal directions in addition to the center of the specimen was measured in each specimen examined. In the microstructure shown in Fig. 4.7, the bright grains are the ferrite phase and the dark ones are the martensite. The grain sizes of the ferrite phase in the DP-steels and the single ferrite phase have been measured and averaged; the DP-steels grain size averages were approximately $13 \mu \mathrm{m}(12.91 \mu \mathrm{m})$ and that in the single ferrite phase is approximately $17 \mu \mathrm{m}(16.95 \mu \mathrm{m})$, using the lineal intercept method as explained in section 4.1.2. The grain size measurements for the DPsteels were carried out in the beginning using both methods explained in section 4.1.2, using equation 4.2 and by the measuring the lengths and deducting the second phase particle intercepts and the total dimension of the total length, and were found to be approximately equal. 


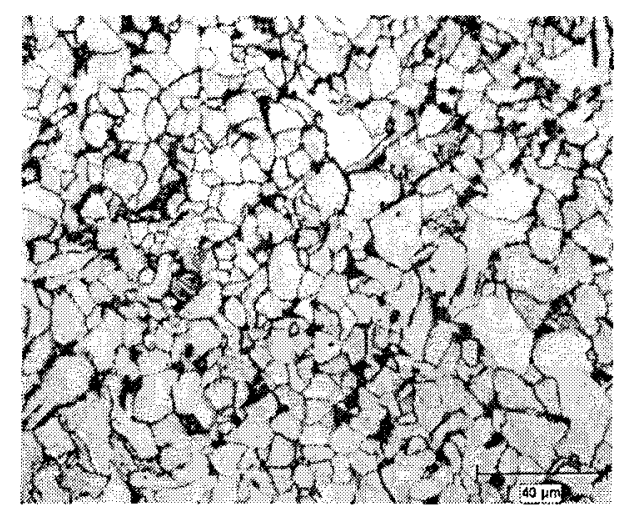

(a)

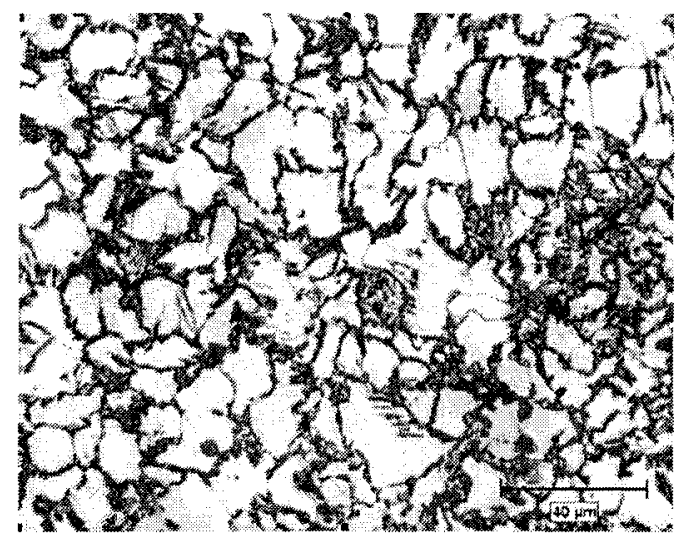

(c-1)

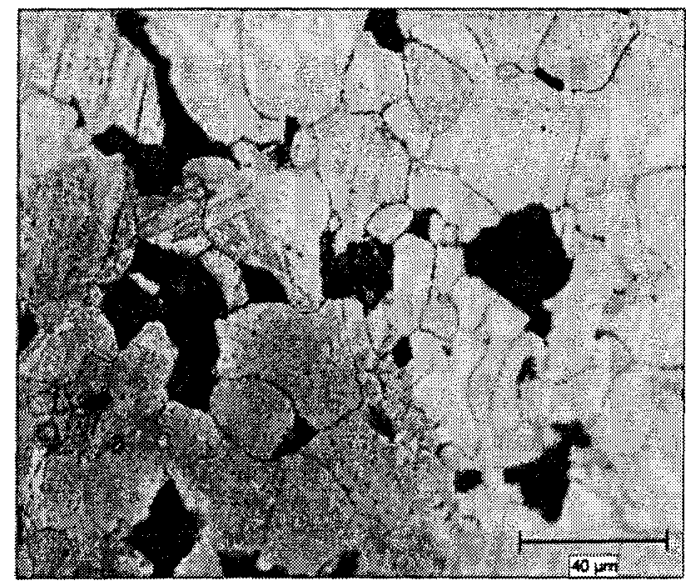

(d-1)

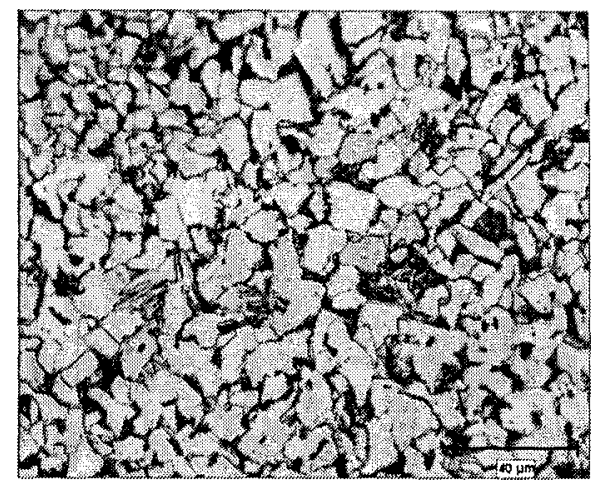

(b)

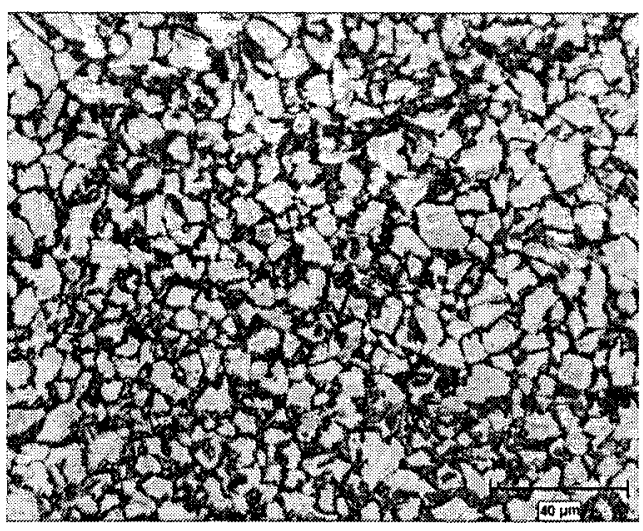

(c-2)

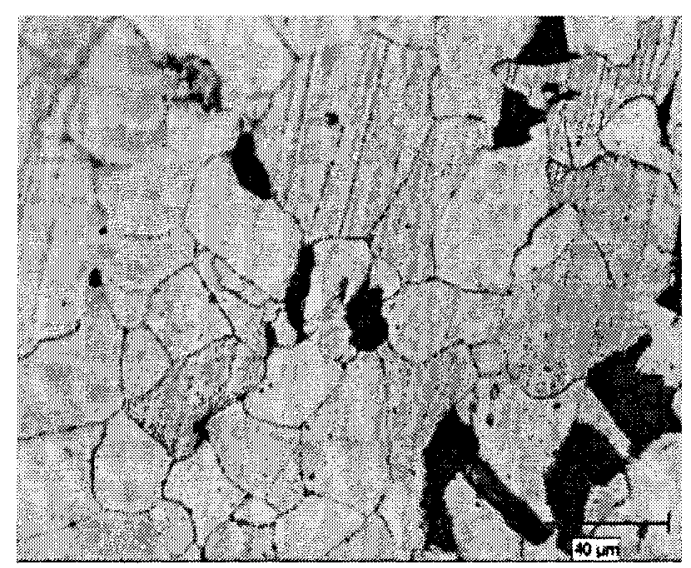

(d-2)

Fig 4.7: Micrographs of typical microstructures produced in this work a) $V_{m}=14 \%$, b) $V_{m}=19.7 \%$, c) $V_{m}=34 \%$ and d) Single ferrite phase with $6 \%$ pearlite. 


\section{CHAPTER}

\section{NUMERICAL MODELING OF DP-STEELS}

In this Chapter the micromechanical modeling of DP-steels will be explored and various attempts investigated to capture the mechanical behavior of the material at hand using several modeling idealizations will be presented. The idealizations used in this work were based on plane strain and axisymmetric models. Modeling the microstructure based on plane strain or axisymmetric cell models reduces the complexity of 3D modeling and minimizes the computational cost. Different idealizations based on plane strain or axisymmetric models are reported in the literature, all of which proved to capture the essential real material behavior to some degree. Huang and Kinloch (1992) and Danielsson et al. (2002) concluded after comparing results of 3D models with previous work, that axisymmetric models could be used without significant loss in accuracy.

In the following sections an investigation of the model, which possesses the intrinsic ability to capture the experimental material behavior reported in the literature will be 
presented. The best model, which displays intrinsic ability to capture DP-steel material behavior, will be used for further development in the next Chapter.

\subsection{Representative Volume Element (RVE):}

The RVE's used in this part of the work are the ones that were shown in Fig. 2.9a based on plane strain (PS1) and axisymmetric (SHA) idealizations and Fig. 2.9b based on plane strain (PS2) idealizations as shown in Chapter 2. The PS2 idealization is developed in order to better capture the interaction between the neighboring hard particles, as previously discussed. The PS3 (Fig. 2.9c) idealization has been reported by Socrate and Boyce (2000) to display very similar results to the PS2 and thus has not been considered. Four-noded, linear, quadrilateral (axisymmetric and plane strain) elements were used. The same mesh is used for the SHA and PS1 idealizations, differing only in element type. Due to symmetry, only the shaded area of Fig. 2.9 is modeled.

In the idealizations considered above, periodic microstructure is assumed. Torquato (2002) has shown that heterogeneous periodic medium will behave like a homogeneous medium, when the material macroscopic responses are considered. Bensoussan et al. (1978) have proven that for structures of periodic microstructures with periodicity much smaller that the length scale of a realization, the problem converges to a solution with a partial differential operator which is called the homogenized operator of a family of periodic partial differential operators. This indicates that the periodic assumption imposed in the micro mechanical model does not enforce periodic responses.

Different micro geometry such as non-circular and concave inclusion shapes as well as irregular geometries can be detected in DP-steels on the two-dimensional view but these have to be imagined as three-dimensional shape inclusions and not in two-dimensional spatial space. In general, the strengthening mechanisms developed in steels to have favorable overall properties suggest microstructures that consists of a soft matrix in which there is a dispersion of round hard particles, rather than needle-like and sharp shape- 
edged shapes, as the round shapes are less likely to initiate cracks (e.g.: Askeland (1985)). Although round shapes are not characteristic of all steel phases, commercial dual phase steels (ferrite-martensite) morphology produced by intercritical annealing, which furnishes a structure of high ductility and strength compared to other heat treatments are nearly round. These are composed of globular martensite particles dispersed in a matrix of ferrite (Kim and Thomas (1981), Balliger and Gladman (1981) Shehata and Crawley (1983)). The same was reported by Tomita (1990) when he used an intercritical heat treatment procedure similar to the one adopted in this work, referring to them as spheroidized and continuous morphologies. The change in the martensite morphology from particle shape to needle-like shape takes place only at prolonged annealing time (more than one hour), which in general is not the case of interest (Kang and Kwon (1987). This can also be seen in the micrographs presented in this work of representative materials used for the different levels of $V_{m}$ (see Fig. 4.7). Furthermore, Shen et al. (1995) presented a study of the effect of the morphology on the behavior of the unit cell models using cylindrical, truncated cylinder, double-cone and spherical shapes of composite reinforced material and concluded that the spherical, and the truncated cylinder with sharp corners shapes give very similar responses. Thus, circular shapes can be idealized since the inclusions are generally nearly round shapes for DP-steels consisting of martensite particles dispersed in a matrix of ferrite which are produced commercially by intercritical annealing.

One of the requirements in an RVE is that it embodies the essence of the microstructure under consideration. The material microstructure of a matrix with a dispersion of hard particles or voids is encountered in different classes of materials including metals, ceramics and polymers. Typically, the second phase particles are not uniform in size and are dispersed in irregular patterns throughout the matrix. Micromechanical modeling of cells assumes an RVE with a periodic arrangement of the second phase particles, which is a simplification intended to solve the problem at low computational cost and reduce the complexity of the problem. In addition, when treating the RVE, as for the ferrite and martensite phases, the constitutive behavior of each is considered isotropic. The ferrite phase (matrix) has preferred slip directions in each crystal or grain but due to the many 
crystals the ferrite contains, which are randomly scattered with differing orientations, it can be considered as an isotropic material. The martensite phase consists of fine lath, which introduce directional behavior (anisotropic), but due to the many martensite grains in the martensite islands or particles, the martensite constitutive behavior can also be considered to be isotropic. These global features of the material are captured in the RVE by imposing isotropic material behavior for the constituents. On the other hand, the RVE is also required to capture the inherent features and morphological features of the microstructure. As mentioned earlier, an RVE should embody the essential features of the microstructure. In developing an RVE the microstructural and related properties, which influence the modeling outcome significantly, should be observed. Although the global material behavior of heterogeneous materials is isotropic, on the microstructural scale this is not true. The martensite packets in a single grain are few and isotropy is not preserved. Thus, in developing the RVE to represent the dual phase steels, isotropy is not considered as a microstructural feature, which need not be realized by the model.

However, the anisotropic nature of the idealization, when chosen to be large enough, should not influence the macroscopic response of the material, which is isotropic in nature. In a discussion of composite materials, Torquato (2002) has presented the following important conclusions. "For composites consisting of isotropic phases, the following general statements can be made: .....However, statistical anisotropy as measured by correlation functions does not necessarily imply a macroscopically anisotropic composite with an effective tensor $\sigma_{\mathrm{e}}$. For instance, composites with cubic symmetry are statistically anisotropic but are macroscopically isotropic (e.g.: cubic lattice with spheres)." Utilizing this fact, in the stochastic moving window approach micromechanical modeling, Jiang et al. (2001) reported that for a triangularly arranged fiber reinforced composite idealization, the orthotropy becomes weaker as the window size increases. When the window becomes large, the apparent elasticity tensor will coincide with the effective one, which is isotropic. 
The physical size of the RVE is taken typically widely as L x L, equal side sizes, (see e.g.: Tverggard (1981), and Socrate and Boyce (2000)) and the radius of the particle is calculated corresponding to the $V_{m}$ considered as shown in Fig. 5.3.

The physical size of the model considering the particle size distribution, which is the subject of the next Chapter, is $\mathrm{Hx} \mathrm{L}$ as will be shown later in Fig $6.3 \mathrm{~b}$ which is $2 \mathrm{~L} \times \mathrm{L}$, the height being about double the radius to incorporate the second small particle.

\subsection{The Constitutive Behavior of Each Material Phase:}

The behavior of each steel phase can be determined by tests on steels consisting of a single phase. This is achievable by heating the steel to the required temperature and then cooling at controlled rates using the time temperature transformation (TTT) diagram to get the desired phase. The single-phase steels can then be tested in tension or compression to obtain the characteristic behavior of a specific phase. The constitutive behaviors of bainite, martensite, pearlite and approximated behavior of ferrite have been reported in the literature (Davies (1978a), Bourell and Rizk (1983) and Ishikawa et al. (2000)). In the micromechanical model, the constitutive behavior of each single constituent on its own will only be required to investigate the aggregate behavior, which is thus far achievable. The interaction of phases (interface boundaries) will be ignored, as it is considerably small, on the order of few atomic sizes, compared to the phases being modeled. Thus the boundary will be considered a cohesive interface, although from the metallurgical viewpoint this is not absolutely true as the boundary between the ferrite and the martensite is an interface between different crystal types which is not a cohesive interface, but from the mechanical point of view, the interface is strong enough to be considered cohesive for the mechanics and mechanism of deformation to be investigated compared to the non-cohesive boundaries. Therefore, a perfectly continuous boundary between the ferrite and martensite has been used in the micro mechanical model. 
The investigations and comparisons made in this Chapter are based on experimental observations, qualitatively, as it is essential at the beginning to establish the promising model(s) in order to avoid continuing working with the ones which would not fundamentally have any capability to address the mechanism of deformation of the material considered in this work. Thus, the single-phase material behavior reported in the literature will be used first to capture the DP-steel material behavior qualitatively. When such a model is developed, single material behavior is then developed in this work from a commercial DP-steel to validate the model results quantitatively.

Each phase is considered to be an elastic plastic solid and it is assumed that the strain increment can be additively decomposed into elastic and plastic components,

$$
d \varepsilon^{t}{ }_{i j}=d \varepsilon_{i j}^{e}+d \varepsilon_{i j}^{p}
$$

Where the elastic component is described by Hooke's law, which is given by:

$$
\varepsilon^{e}{ }_{i j}=\frac{\sigma_{i j}}{2 G}-\frac{v}{E} I_{1} \delta_{i j}
$$

Where $\delta_{i j}$ is the kronecker delta already defined in section 3.2.1, $E$ is the Young's modulus, $v$ is Poisson's ratio, $\mathrm{G}$ is the shear modulus which is related to $\mathrm{E}$ and $v$ by:

$$
G=\frac{E}{2(1+v)}
$$

And $I_{1}$ is the first invariant of stress tensor:

$$
I_{1}=\sigma_{k k}
$$


The plastic strain rate is given as:

$$
\begin{array}{ll}
d \varepsilon_{i j}^{p}=0 & f<0 \\
d \varepsilon_{i j}^{p}=\frac{3}{2} \frac{d \varepsilon_{e}{ }^{p}}{\sigma_{e}} \sigma_{i j}^{\prime} & f=0
\end{array}
$$

Where the deviatoric stresses $\sigma_{i j}^{\prime}=\sigma_{i j}-\frac{1}{3} \sigma_{k k}$ and the effective stress, $\sigma_{e}$, and the effective plastic strain rate, $d \varepsilon_{e}{ }^{p}$, are defined as:

$$
\begin{aligned}
& \sigma_{e}=\sqrt{\frac{3}{2} \sigma_{i j}^{\prime} \sigma_{i j}^{\prime}} \\
& d \varepsilon_{e}^{p}=\sqrt{\frac{2}{3} d \varepsilon_{i j}^{p} d \varepsilon_{i j}^{p}}
\end{aligned}
$$

The von Mises yield condition is assumed:

$$
f=\sigma_{e}-\bar{\sigma}
$$

where $\bar{\sigma}$ is a function of the effective plastic strain and is taken to describe the isotropic hardening.

The hardening behavior of the two phases is taken from the experimental results obtained by Davies (1978) and expressed by the following:

$$
\begin{aligned}
& \bar{\sigma}_{f}=K_{f}\left(\varepsilon_{0}+\varepsilon_{f}^{p}\right)^{n_{f}} \\
& \bar{\sigma}_{m}=K_{m}\left(\varepsilon_{0}+\varepsilon_{m}^{p}\right)^{n_{m}}
\end{aligned}
$$


Where the subscripts $f$ and $m$ denote ferrite and martensite, respectively, $\varepsilon_{0}$ is taken to be 0.002 in this work and $n_{m}$ and $n_{f}$ are taken as 0.07 and 0.31 , respectively (Davies (1978a)). A power law relation in which the values of the ultimate stress for the single ferrite and martensite phases and the values of $n_{m}$ and $n_{f}$ reported by Davies (1978a) are utilized to get $K_{m}$ and $K_{f}$ which were found to be $2409 \mathrm{MPa}$ and $597 \mathrm{MPa}$, respectively.

The stress vs. plastic strain for each phase is shown in Fig. 5.1.

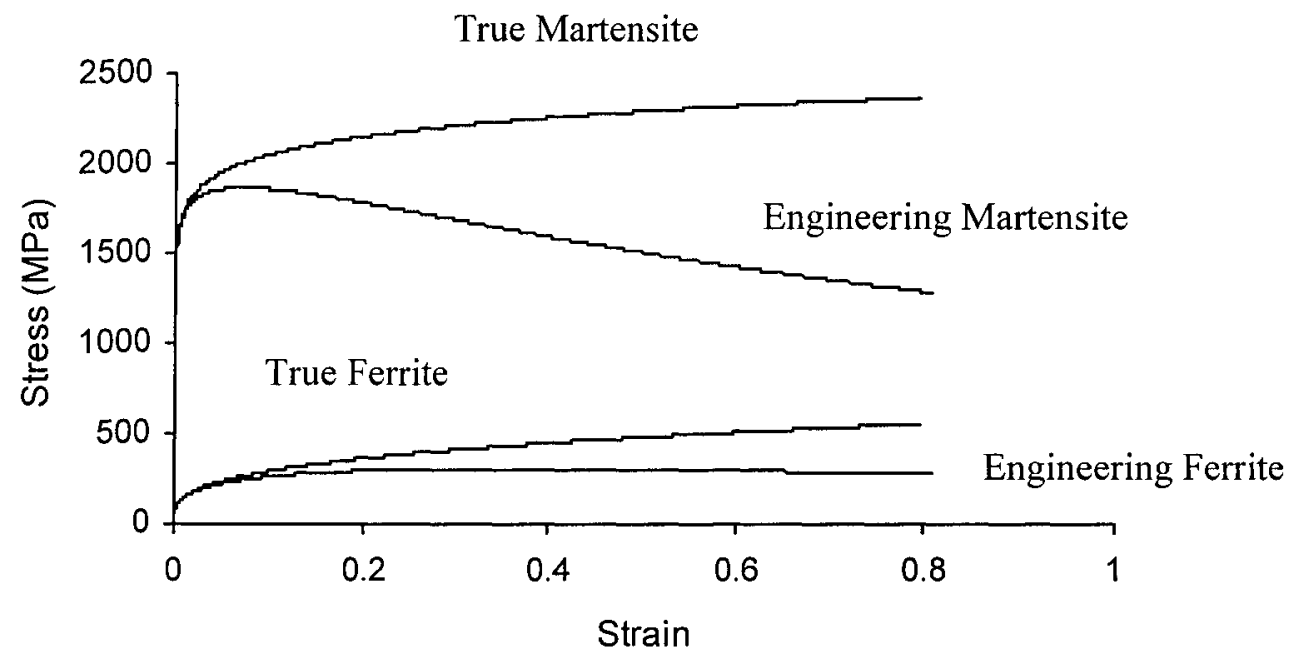

Fig. 5.1: Idealized behavior of the martensite and ferrite phases shown as true stress vs. true strain and engineering stress vs. engineering strain for uniaxial tensile stress conditions.

To better illustrate the difference in uniform elongation between the two phases, the behavior under uniaxial tension stress is considered. Under these conditions, the effective stress reduces to the true uniaxial stress, $\sigma$, and the effective plastic strain is equal to the plastic strain along the direction of loading or true strain, $\varepsilon$. The corresponding engineering quantities are related to the true quantities as follows: 


$$
\begin{aligned}
& e=\exp (\varepsilon)-1 \\
& \sigma_{n}=\sigma /(1+e)
\end{aligned}
$$

The engineering stress vs. engineering strain response for uniaxial tension loading is also shown in Fig. 5.1, where the difference in uniform strain (strain at maximum engineering stress) for the two phases is quite apparent. ${ }^{1}$

The continuity assumption, which is considered in this work is explained well in what Torquato (2002) says “...in which the microscopic scale is much larger than the molecular dimensions but much smaller than characteristic length of the macroscopic sample. In such circumstances, the heterogeneous material can be viewed as a continuum on the microscopic scale, subject to classical analysis, and macroscopic or effective properties can be ascribed to it." The assumption of the elastic plastic response of the constituents is based on the fact that they can be considered continuum parts in the model. Comparing the predicted responses obtained numerically to the experimental results, which will be shown in Chapter 7, validates this assumption. At the microstructural scale, the ferrite phase experiences large plastic strains especially at the ferrite-martensite interface, which is reported experimentally by Shen et al. (1986) and Rashid and Cprek (1978).

\subsection{Homogenization Method:}

The macroscopic stress components are computed as the volume average of the microscopic components according to the following equations:

\footnotetext{
${ }^{1}$ It should be noted that no fracture criteria has been employed so the response shown cannot predict the limiting strain value of the phase under tensile loading.
} 


$$
\begin{aligned}
& S_{i j}=\frac{1}{V} \int_{V} \sigma_{i j} d V \\
& E_{i j}=\frac{1}{V} \int_{V} \varepsilon_{i j} d V
\end{aligned}
$$

Where $S_{i j}$ and $E_{i j}$ are the macroscopic average component of stresses and strains over the microscopic volume of the micro mechanical model. As mentioned in Chapter 2 the macro mechanical behavior of the aggregate is, therefore, approximated by the volume average of the micro mechanical behavior.

\subsection{Rule of Mixtures:}

The most straightforward homogenization techniques are known as the rule of mixtures, which are of different forms. The simplest of these assume either uniform strain, known as the Voigt estimate, or uniform stress, which is known as the Reuss estimate. The two estimates have been shown by Hill (1963) to be the upper and the lower bounds, respectively. Using equation 5.6, the Voigt and Reuss bounds can be written as:

$$
\begin{aligned}
& \bar{\sigma}_{c}=V_{m} K_{m}\left(\varepsilon_{0}+\varepsilon_{m}^{p}\right)^{n_{m}}+\left(1-V_{m}\right) K_{f}\left(\varepsilon_{0}+\varepsilon_{f}^{p}\right)^{n_{f}} \\
& \varepsilon_{c}^{p}+\varepsilon_{0}=V_{m}\left(\frac{\bar{\sigma}_{m}}{K_{m}}\right)^{1 / n_{m}}+\left(1-V_{m}\right)\left(\frac{\bar{\sigma}_{f}}{K_{f}}\right)^{1 / n f}
\end{aligned}
$$

Where the subscript ' $c$ ' denotes the aggregate. As shown in Fig. 5.2, for low martensite volume fractions the two bounds are relatively close, but at higher volume fractions the discrepancy is quite large.

A modified form of the rule of mixtures was proposed by Bourell and Rizk (1983). The ferrite part contains a term, which accounts for the influence of the austenite-martensite induced transformation strain on the ferrite matrix. They have shown that this has 
maximum effect of about $2 \%$ on ranges of the martensite volume fraction of $30 \%$ to $50 \%$ in ferrite-martensite dual phase steel. The modified rule of mixtures is of the form:

$$
\sigma_{c}=V_{f} K_{f}\left(\varepsilon_{e}+\varepsilon_{d m}\right)^{n_{f}}+V_{m} K_{m} \varepsilon_{e}^{n_{m}}
$$

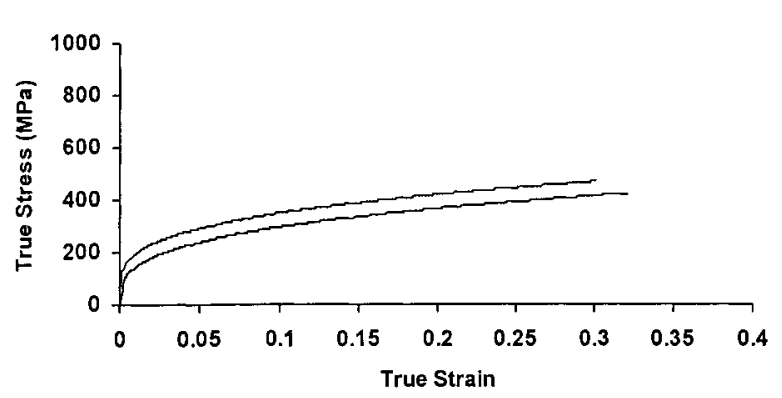

(a)

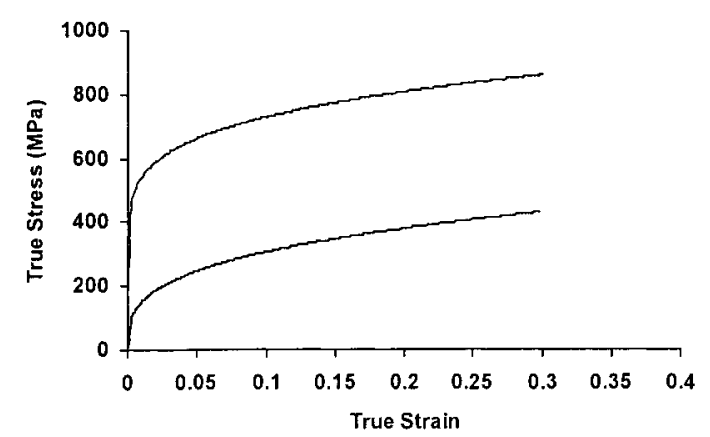

(c)

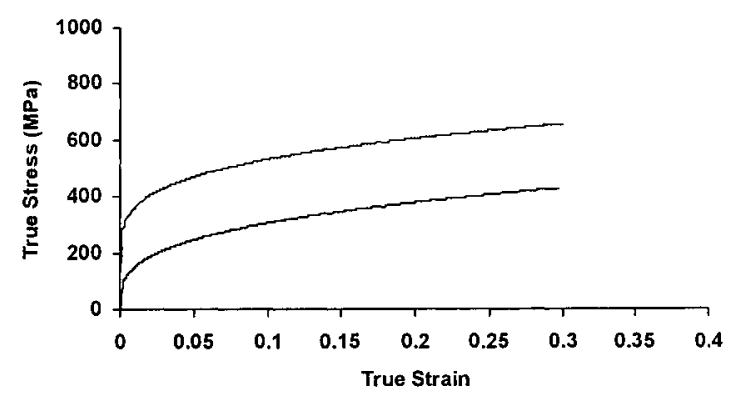

(b)

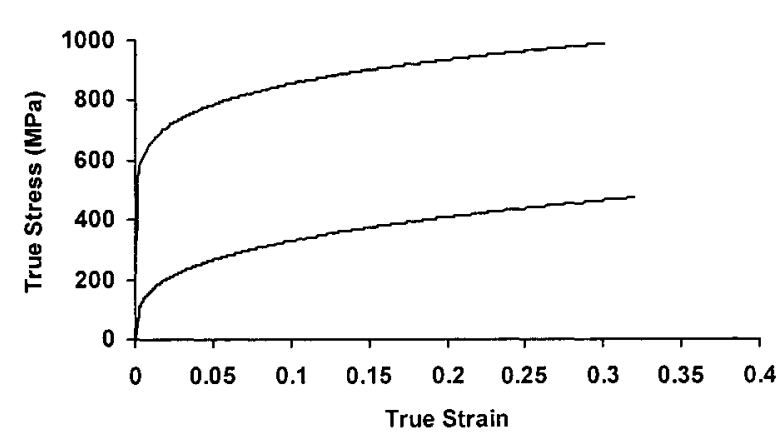

(d)

Fig. 5.2: Upper and lower bound predicted by ROM for several volume fractions $V_{m}$ a) $3.2 \%$ b) $13.6 \%$ c) $24.8 \%$ and d) $32 \%$

Where $\sigma_{c}$ is the effective microscopic composite stress and $\varepsilon_{d m}$ is the induced microscopic austenite-martensite transformation strain; $\varepsilon_{e}$ is the effective microscopic 
composite strain; $K_{f}$ and $K_{m}$ are the Hollomon strength coefficients for the ferrite and martensite, respectively; $n_{f}$ and $n_{m}$ are the strain hardening coefficients of the ferrite and martensite, respectively; $V_{f}$ and $V_{m}$ are the volume fractions of the ferrite and martensite respectively in the composite material. The ferrite part contains a term, which accounts for the influence of the austenite-martensite induced transformation strain on the ferrite matrix. This equation was also used to investigate the upper and lower bound of the stress vs. strain trend, as was done in Fig. 5.2. The results obtained using the modified rule of mixtures with the prestrain induced in the ferrite matrix by the austenite-martensite transformation at different volume fractions of the harder phase show that the difference between the ordinary and modified rule of mixtures to be negligible at low $V_{m}$ and about $1 \%-2 \%$ at high $V_{m}$. The large discrepancy in the upper and lower bound in either case demonstrates the need to use methods other than the rule of mixtures for predicting the aggregate response.

Different straining models based on rule of mixtures have been used in the past by various authors in analyzing the strength of DP-steels. Two main assumptions were made, one assumes the material as a mixture of two ductile phases deforming at the same ratio, also called isostrain models. On the other hand, the second assumption made by some workers followed Ashby's theory of particle strengthening (Balliger and Gladman (1981). Others like Speich and Miller (1979) and Szewczyk and Gurland (1982) considered the difference between the strain in the martensite and ferrite but held the strain ratio constant throughout the entire tensile process. A comprehensive review of the above is given by Szewczyk and Gurland (1982) and Korzekwa et al. (1980). Elasticplastic behavior of two-phase polycrystalline materials have also been predicted by using the Mori-Tanaka (1973) method, which is used as the basis for many continuum analytical models (e.g.: Weng (1990), Tomota and Kuroki (1976) and Rudiono and Tomota (1997)).

Korzekwa et al. (1980) presented a detailed evaluation of the methods such as the rule of mixtures, isostrain assumption, strain partitioning models (also called continuum models) 
and the isostress models. They asserted that although most of the treatments predict the increase in strength and decrease in ductility that accompany an increase in $V_{m}$ reasonably well, some of the correlations may be fortuitous as the basic assumption used to model the strain distribution between the ferrite and martensite are inconsistent with experimental microscopic observations they presented. Ostrom (1981) in a study of the analytical models concluded that without knowledge of how stress and strain develop in the two phases during deformation, no conclusive results could be reached and thus the models based on rule of mixtures are not reliable. In addition, the traditional simulations of the deformation and fracture of solids by application of continuum mechanics and averaged macro parameters or homogenization methods are not sufficient for developing a predictive theory of deformed solids. Internal microstructure evolution should also be considered as they affect the mechanical properties of materials substantially. Local values of the elastic-plastic parameters at the micro level differ widely from the averaged macro data, a fact widely validated and accepted. The computational micromechanical methods provide these local data.

\subsection{Finite Element Modeling Results:}

Finite element analysis, using several different micro mechanical models, has been used to carry out the homogenization procedure. The analyses considered were limited to $2 \mathrm{D}$, plane strain and axisymmetric cases to keep computational time reasonable. The analysis was performed using the commercial code ABAQUS. Each phase, namely martensite and ferrite, is considered to be an elastic-plastic solid as described by equations. 5.1-5.6, with $\mathrm{E}=200 \mathrm{GPa}$ and $v=0.3$.

Referring to Fig. 5.3, the volume fraction, $V_{m}$, is computed as $\pi \mathrm{a}^{2} / 4 \mathrm{~L}^{2}$ and $2 / 3 \mathrm{a}^{3} / \mathrm{L}^{3}$ for the plane strain and axisymmetric cases, respectively. Referring to the same figure, symmetry boundary conditions are used for sides $\mathrm{S} 1$ and $\mathrm{S} 2$, while side $\mathrm{S} 3$ has a uniform 
displacement in the $x_{1}$ direction and side $\mathrm{S} 4$ has uniform displacement in the $x_{2}$ direction. This was achieved using an equation command available in ABAQUS.

The boundary condition applied on the unit cell model simulates the loading condition, which the microstructure experiences when loaded under uniaxial tension. The uniform strain boundary condition on the top part of the model is the applied displacement that the microstructure experiences from the far field applied load similar to the loading condition in a tensile bar test considered in this work. To account for the influence of the neighboring particles (of other unit cells) symmetry is enforced by constraining the RVE to remain a circular cylinder throughout the analysis. The circular cylindrical cell surrounding each particle is required to remain circular and within each cell, symmetry is assumed about the cell centerline and thus only the area identified in the models need be analyzed. This symmetry condition and the natural use of axisymmetric elements for a cylinder to model the cell impose logical use of above boundary conditions and not periodic ones, which can't be applied to axisymmetric unit cells.

The boundary conditions imposed on the model can also be stated mathematically for the plane strain and axisymmetric unit cells as follows:

Referring to Fig. 5.3, for plane strain cases the following boundary conditions are applied:

$$
\begin{gathered}
\mathrm{u}_{1}\left(0, x_{2}\right)=0, \mathrm{~T}_{2}\left(0, x_{2}\right)=0 \\
\mathrm{~T}_{1}\left(L, x_{2}\right)=0, \mathrm{~T}_{2}\left(L, x_{2}\right)=0 \\
\mathrm{u}_{2}\left(x_{1}, 0\right)=0, \mathrm{~T}_{1}\left(x_{1}, 0\right)=0 \\
\mathrm{u}_{2}\left(x_{1}, L\right)=\mathrm{U}_{2},
\end{gathered}
$$

For axisymmetric cases the following boundary conditions are applied: 


$$
\begin{array}{r}
\mathrm{u}_{1}\left(0, x_{2}\right)=0, \mathrm{~T}_{2}\left(0, x_{2}\right)=0, \mathrm{~T}_{3}\left(0, x_{2}\right)=0 \\
\mathrm{~T}_{1}\left(L, x_{2}\right)=0, \mathrm{~T}_{2}\left(L, x_{2}\right)=0, \mathrm{~T}_{3}\left(L, x_{2}\right)=0 \\
\mathrm{u}_{2}\left(x_{1}, 0\right)=0, \mathrm{~T}_{1}\left(x_{1}, 0\right)=0, \mathrm{~T}_{3}\left(x_{1}, 0\right)=0 \\
\mathrm{u}_{2}\left(x_{1}, L\right)=\mathrm{U}_{2}, \mathrm{~T}_{1}\left(x_{1}, L\right)=0, \mathrm{~T}_{3}\left(x_{1}, L\right)=0
\end{array}
$$

Where $\mathrm{T}_{i}$ stands for tractions and $\mathrm{u}_{i}$ stands for displacements imposed in the ith coordinate direction.

Obviously, periodic boundary conditions can't be applied to the axisymmetric unit cells but can be applied to the plane strain cases. However, in order to compare the response of the models, the same boundary conditions applied to the axisymmetric unit cells are applied to the plane strain cases.

In this work we are interested in loading the material using uniform displacement boundary condition at the sides of the unit cell simulating the experimental procedure followed. In addition, to assure that the material macroscopically behaves as a von Mises material description the same loading condition as the case of loading a tensile bar is imposed to get the same effective stress-strain responses for the deformation behavior of the material.

The aggregate strains are computed as:

$$
\begin{aligned}
& E_{11}=\ln \left(u_{1}\left(L, x_{2}\right) / L\right) \\
& E_{22}=\ln \left(u_{2}\left(x_{1}, L\right) / L\right)
\end{aligned}
$$

The engineering normal stress in the $x_{2}$ direction is computed from the resultant force divided by the original area, from which using equation 5.7, the Cauchy or true stress component is computed. For the SHA micro models the state of stress is uniaxial, such that the $S_{22}$ and $E_{22}$ components of stress and strain are equal to the corresponding 
effective quantities. For the plane strain case $\left(E_{33}=0\right), S_{33}$ is well approximated by the intermediate value of the other normal stresses $\left(S_{33}=\frac{S_{11}+S_{22}}{2}\right)$ after a plastic strain a few times the strain at yield (Hill (1950)). Since $S_{11}=0, S_{33}$ is taken to be $\frac{S_{22}}{2}$ and the effective stress and strain are computed from equation 5.4.

Each mesh has also been subjected to both prescribed traction and displacement boundary conditions along side S4 (See Figure 5.3). For a properly developed RVE, the homogenization procedure should yield the same effective stress vs. effective strain as described by Hill (1963). The comparison for PS1 for a $V_{m}$ of $11.8 \%$ is shown in Fig. 5.4. Similar results were obtained for the other two models, which clearly indicate a properly developed RVE as per Hill's description.

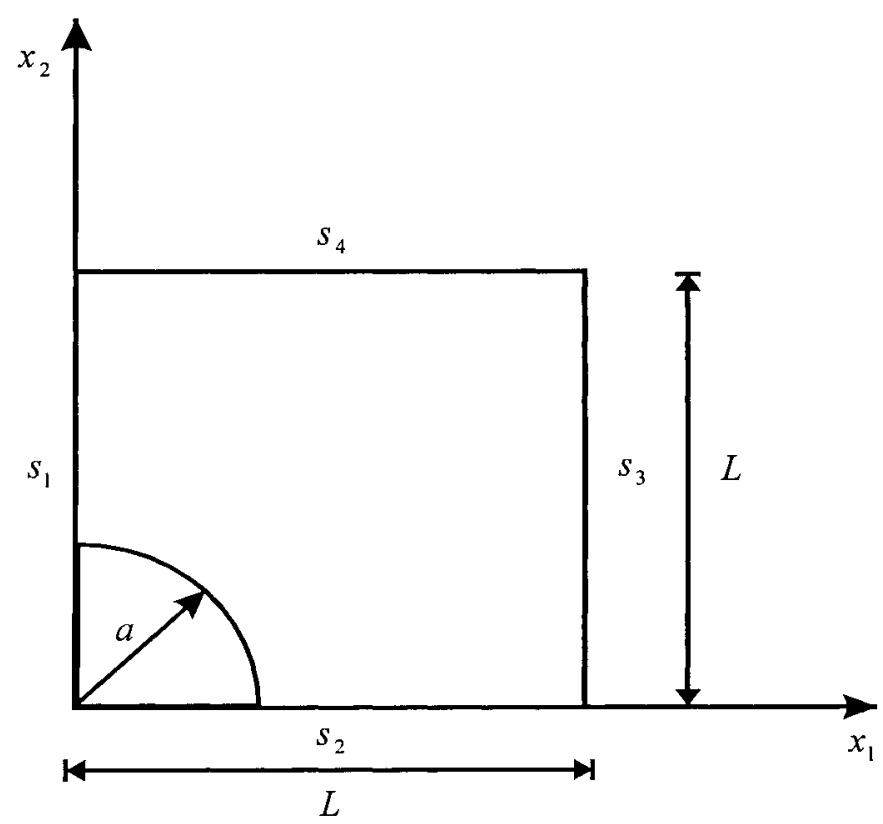

Fig. 5.3: Dimensions of the micro mechanical model.

The predicted response for the aggregate is computed for each case for varying martensite volume fractions. Results are shown in Fig. 5.5 in terms of engineering stress vs. strain to 
better show the anticipated decrease in uniform elongation with increasing volume fraction.

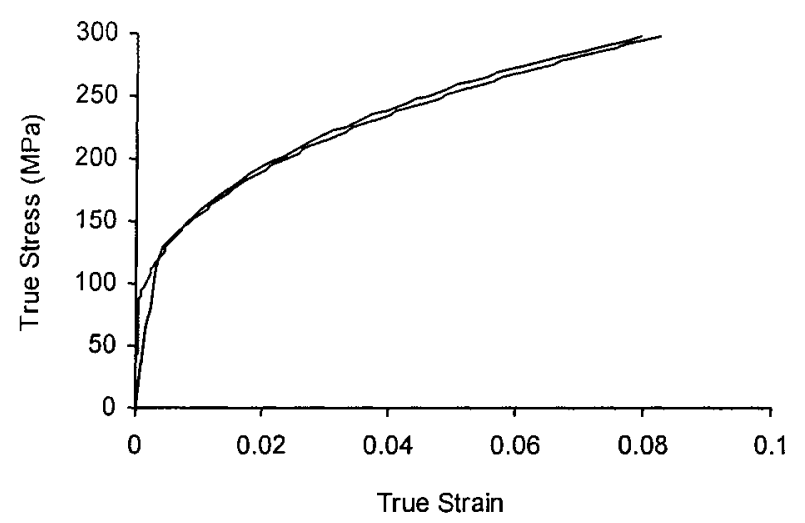

Fig. 5.4: Comparison of behavior predicted for the aggregate material using traction and displacement boundary conditions on side $\mathrm{S}_{4}$ for the PS1 model and $V_{\mathrm{m}}=\mathbf{1 1 . 8 \%}$.

As shown in Fig. 5.6 the response given for each model is quite similar at low volume fractions. However significant differences become apparent for volume fractions above approximately $23 \%$. Figure 5.7 shows the results for the different models at an approximate volume fraction of $32-36 \%$. The response for the axisymmetric SHA model is quite similar and consistent with experimentally observed behavior, i.e. engineering stress increases up to a maximum, which occurs at the onset of instability. The plane strain models appear to overpredict strain hardening, which unrealistically suppresses the localization. This can be seen in Fig. $5.5 \mathrm{~b} \& \mathrm{c}$, where increasing $V_{m}$ results in increasing hardening, which is contradictory to what is observed experimentally (Bag et al. (1999), Tomita (1990), Tomota (1987), Shen et al. (1986)).

The evolution of effective plastic strain for the axisymmetric SHA, PS1 and PS2 idealizations are shown in Fig. 5.8, Fig. 5.9 and Fig. 5.10 corresponding to nominal strains of $10 \%, 20 \%$ and $30 \%$ for volume fractions of about $13 \%, 23 \%$ and $35 \%$ respectively. All three models show the heterogeneous distribution of strain, which agrees with experimental observations of Shen et al. (1986) and Rashid and Cprek (1978). 
However, only the axisymmetric SHA model shows plastic strain extending into the martensite which gets triggered at $V_{m}=23 \%$ as shown in Fig. 5.9 c. In both plane strain models the martensite remains elastic at all levels of $V_{m}$ as can be seen in Fig. $5.8 \mathrm{a} \& \mathrm{~b}$, Fig. $5.9 \mathrm{a} \& b$ and Fig. $5.10 \mathrm{a} \&$ b. At $V_{m}$ lower than about $23 \%$ there was no plastic deformation extending into the martensite phase in all the models including the axisymmetric model as shown in Fig. 5.8 a, b \& c. The onset of the difference in the prediction of the models starts when the plastic deformation in the martensite takes place. Although the amount of plastic strain in the martensite is quite small $(<8 \%)$, the effect on the overall material response is significant. This is demonstrated quite clearly in Fig. 5.11, which compares the response in the axisymmetric case treating the martensite as an elastic solid to that when the martensite is considered an elastic-plastic solid. Treating the material as an elastic solid results in a response, which is, quite similar to that observed in the plane strain models, and contrary to experimental observations. The plastic deformation of the martensite is, therefore, judged to be quite important and must necessarily be captured by an appropriate micro mechanical model. In the case of the constituent behavior considered here, this only occurs for the axisymmetric model. 


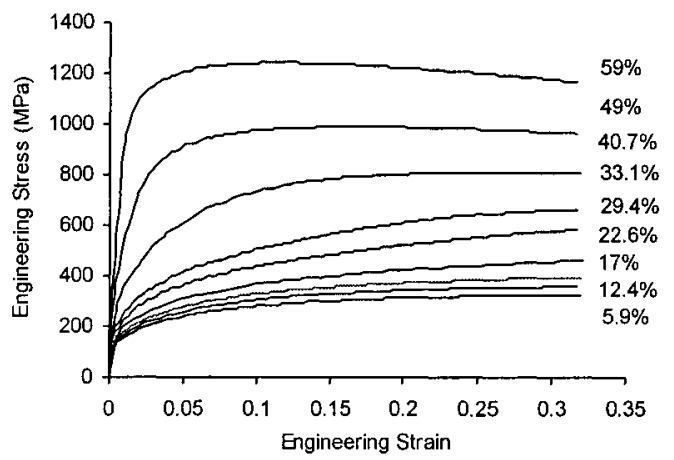

(a)

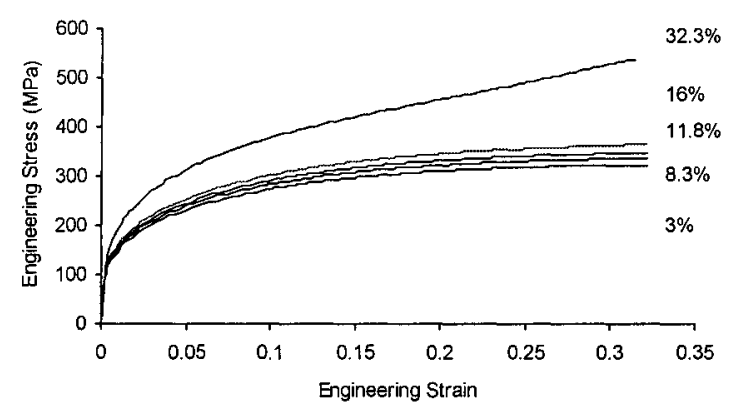

(b)

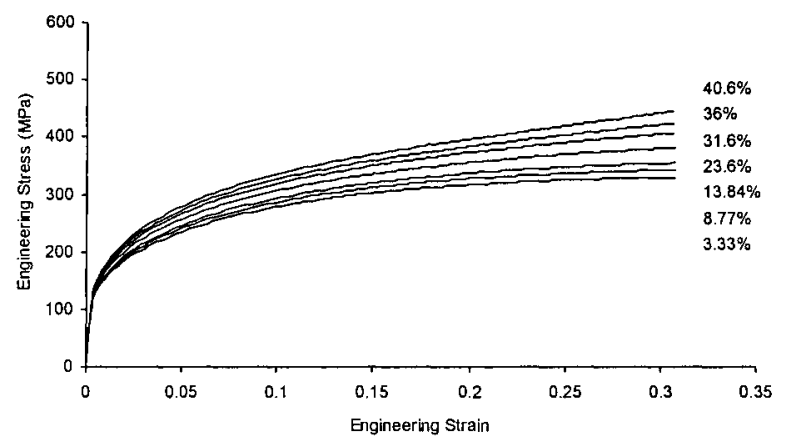

(c)

Fig. 5.5 Computed engineering stress vs. engineering strain under uniaxial tensile stress conditions for varying $V_{m}$ a) SHA model b) Plane strain (PS1) model c) Plane strain (PS2) model. 


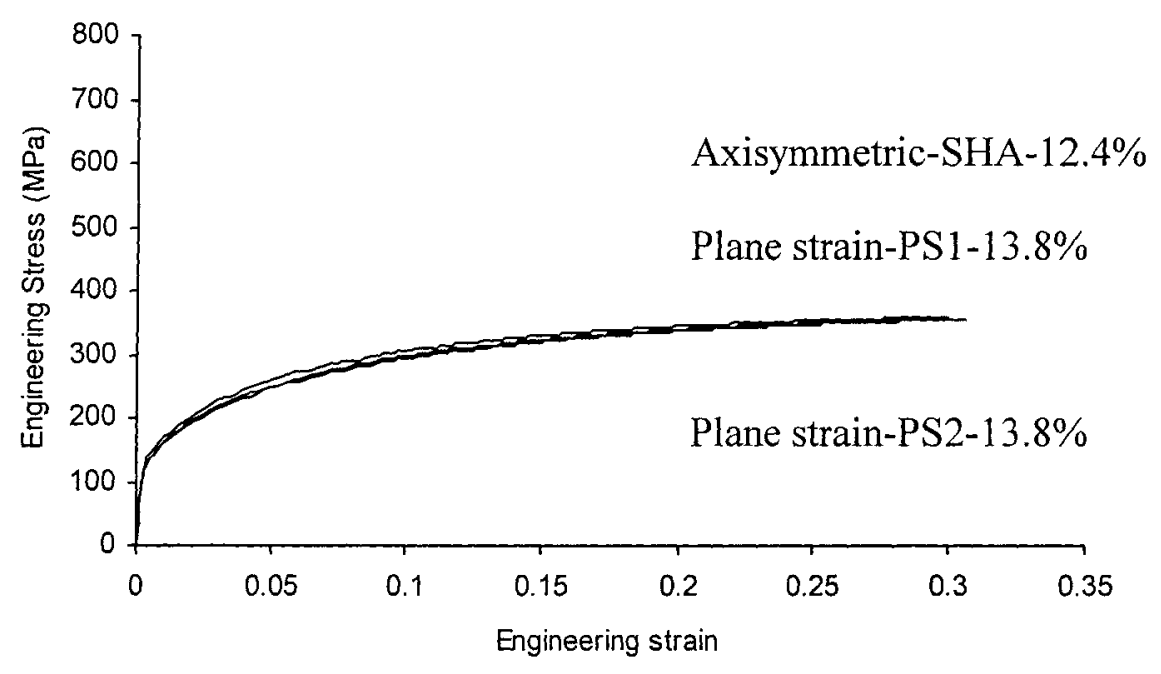

Fig. 5.6: Comparison of computed behavior using different models for low martensite volume fraction.

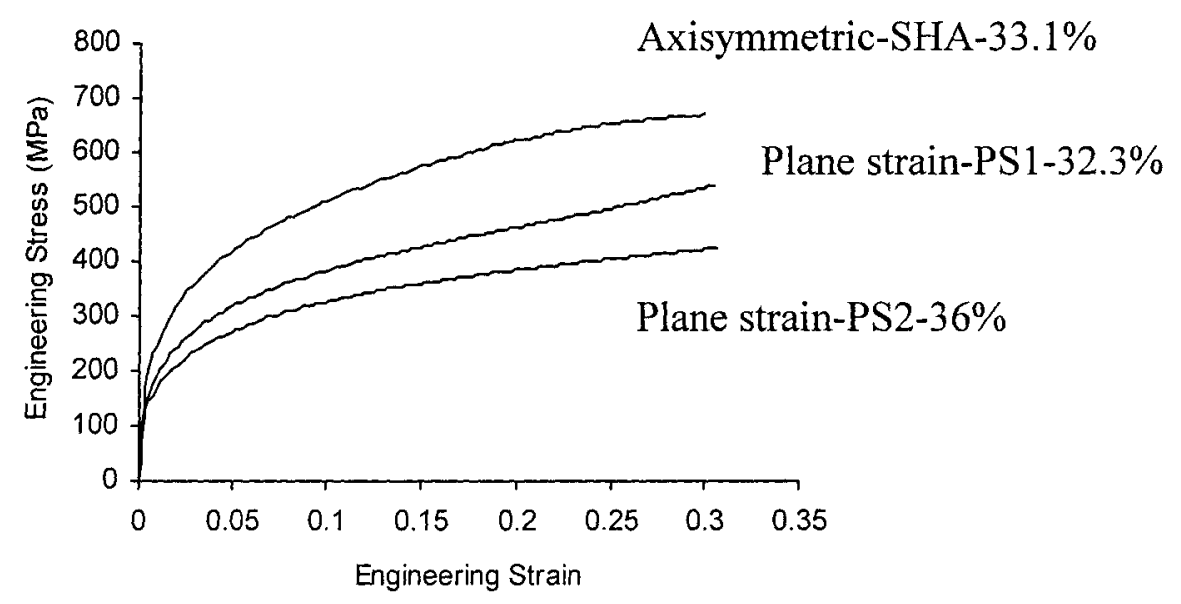

Fig. 5.7: Comparison of computed behavior using different models for high martensite volume fraction. 

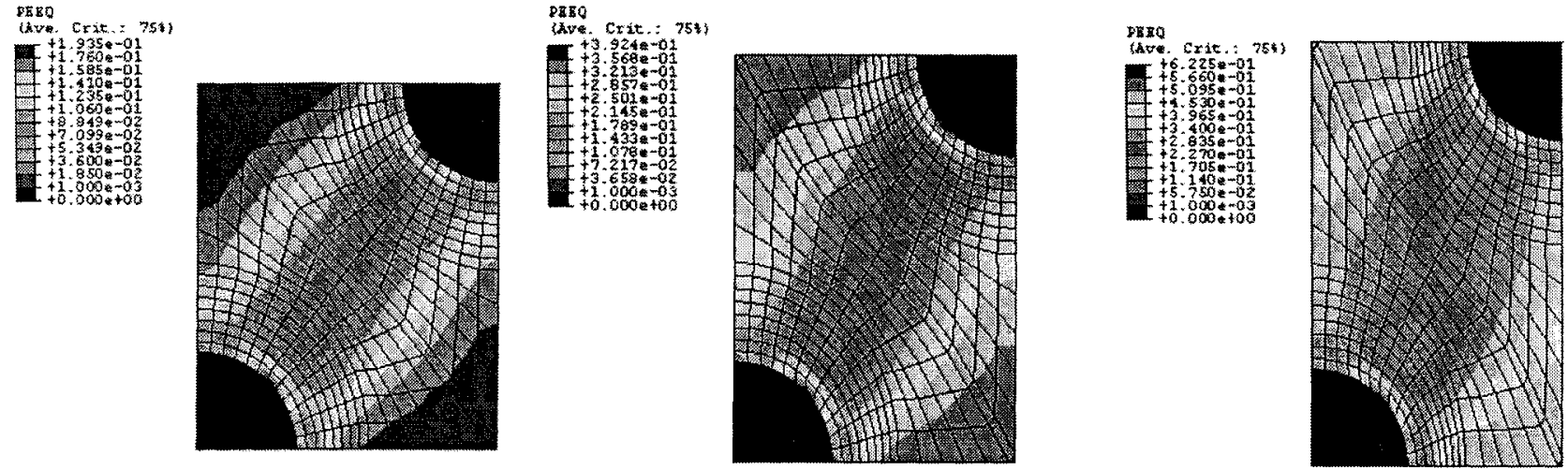

(a)
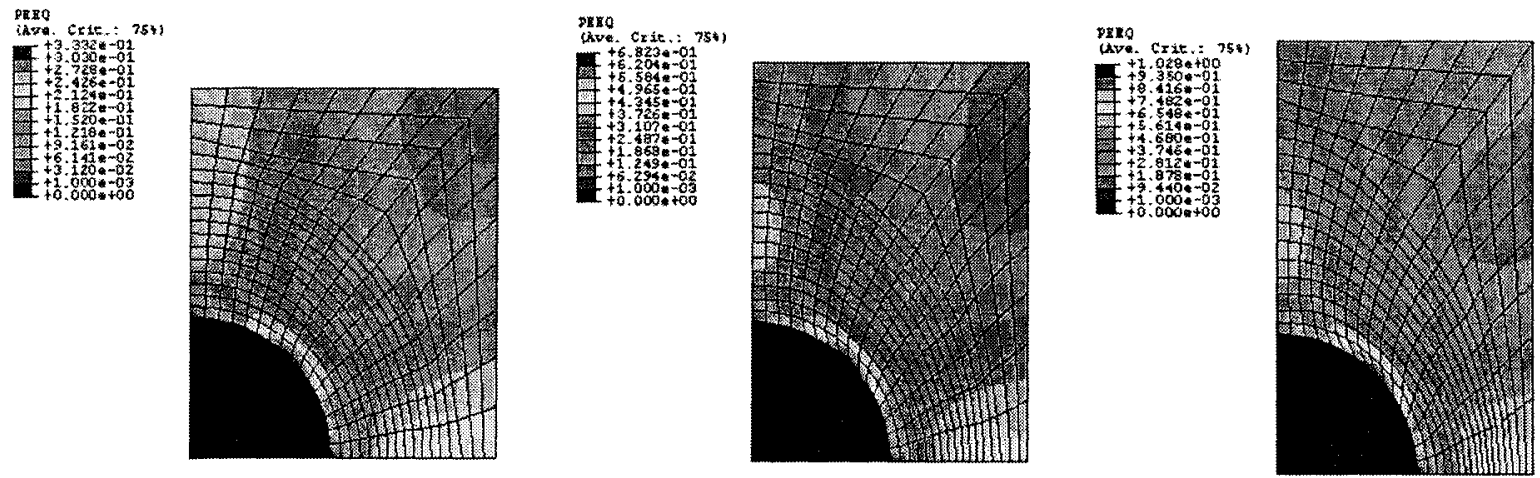

(b)
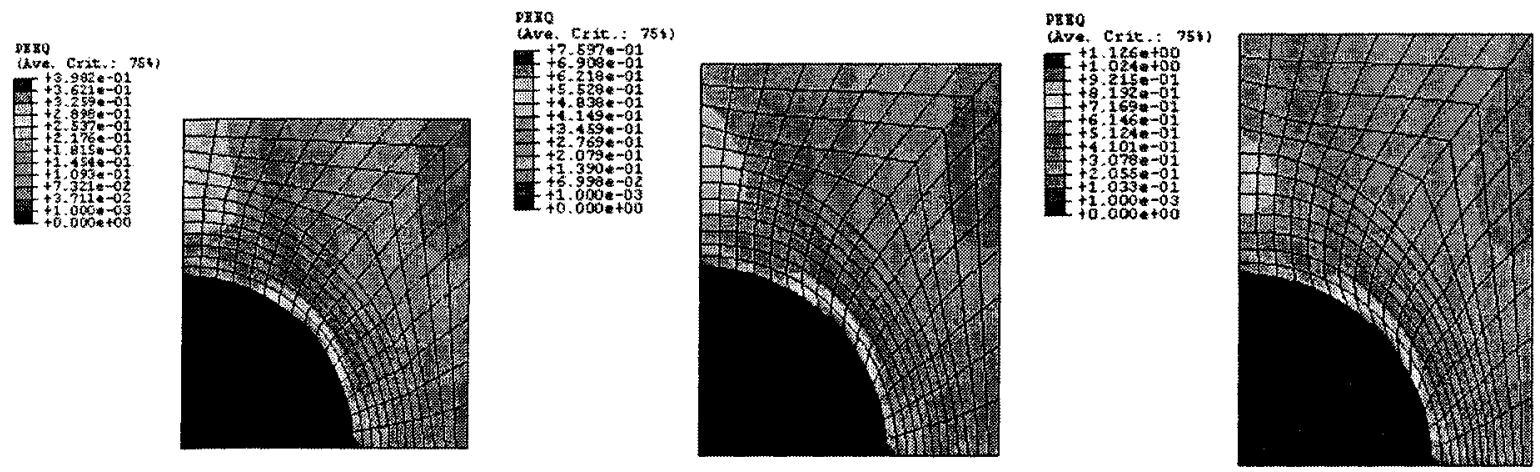

(c)

Fig. 5.8: Contours of effective plastic strain for nominal strains of $10 \%, 20 \%$, and $30 \%$ and $V_{m} \approx 13 \%$ a) Plane strain (PS2) model b) Plane strain (PS1) model c) SHA axisymmetric model. 

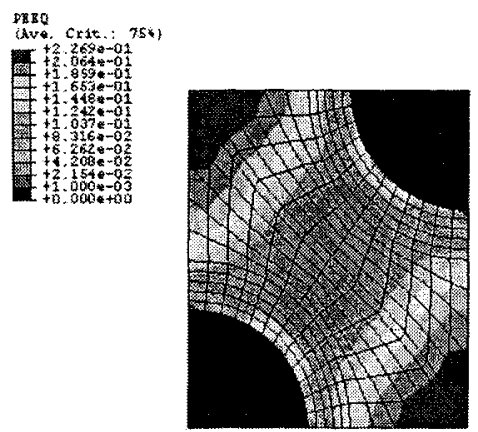

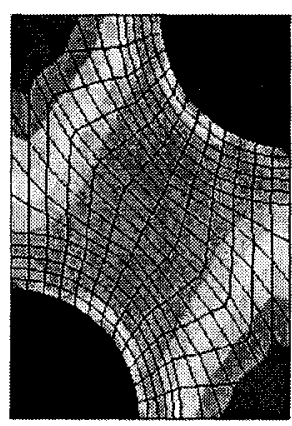

(a)
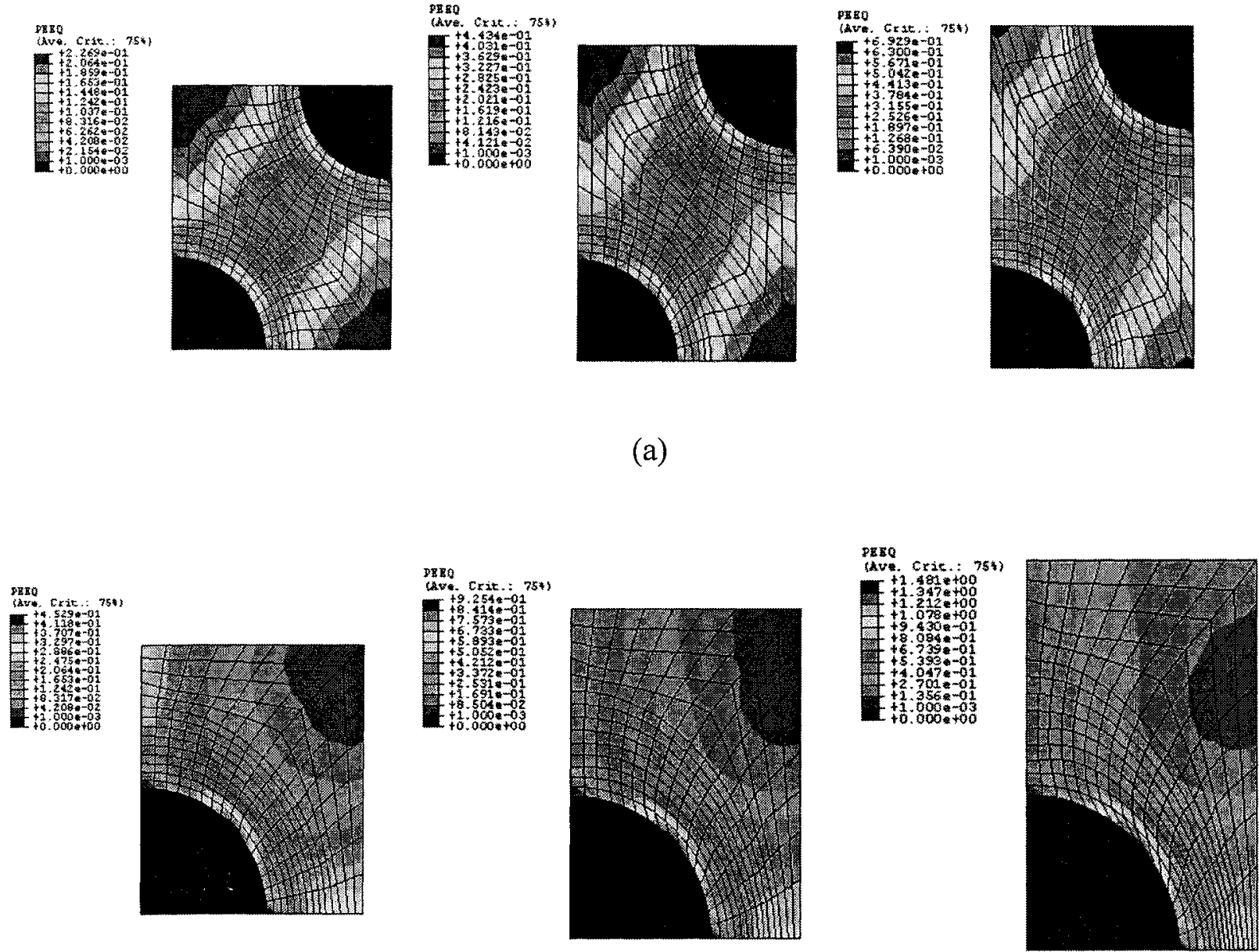

(b)
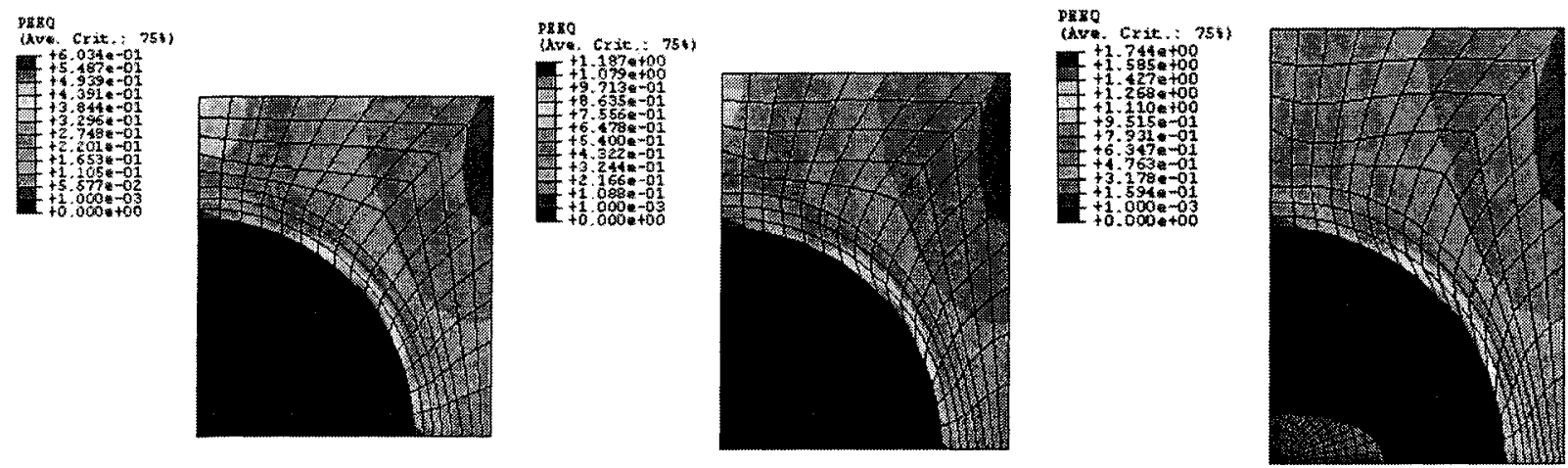

(c)

Fig. 5.9: Contours of effective plastic strain for nominal strains of $10 \%, 20 \%$, and $30 \%$ and $V_{m} \approx 23 \%$ a) Plane strain (PS2) model b) Plane strain (PS1) model c) SHA axisymmetric model. 

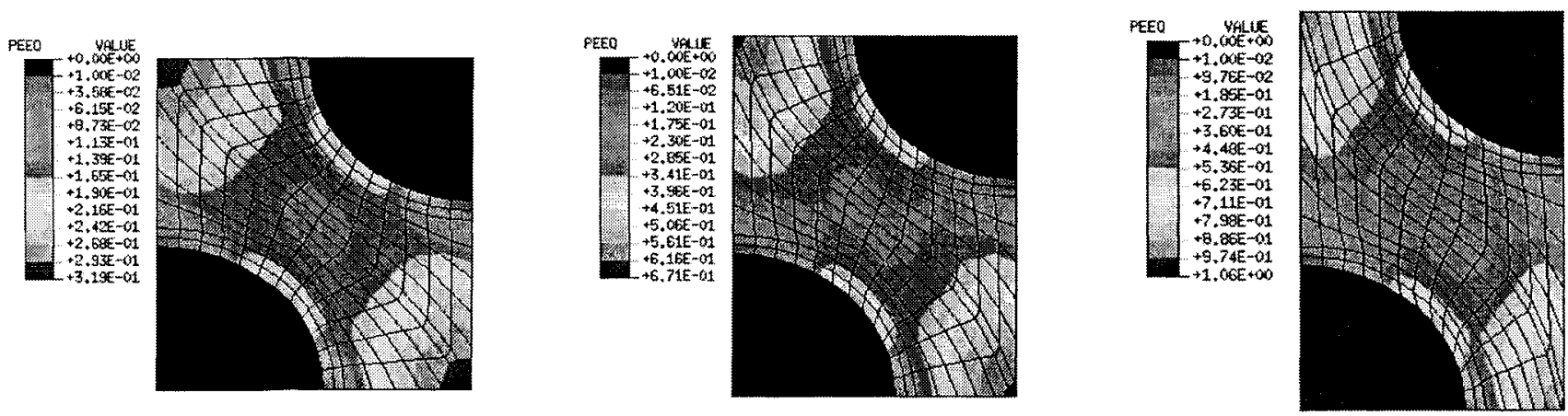

(a)
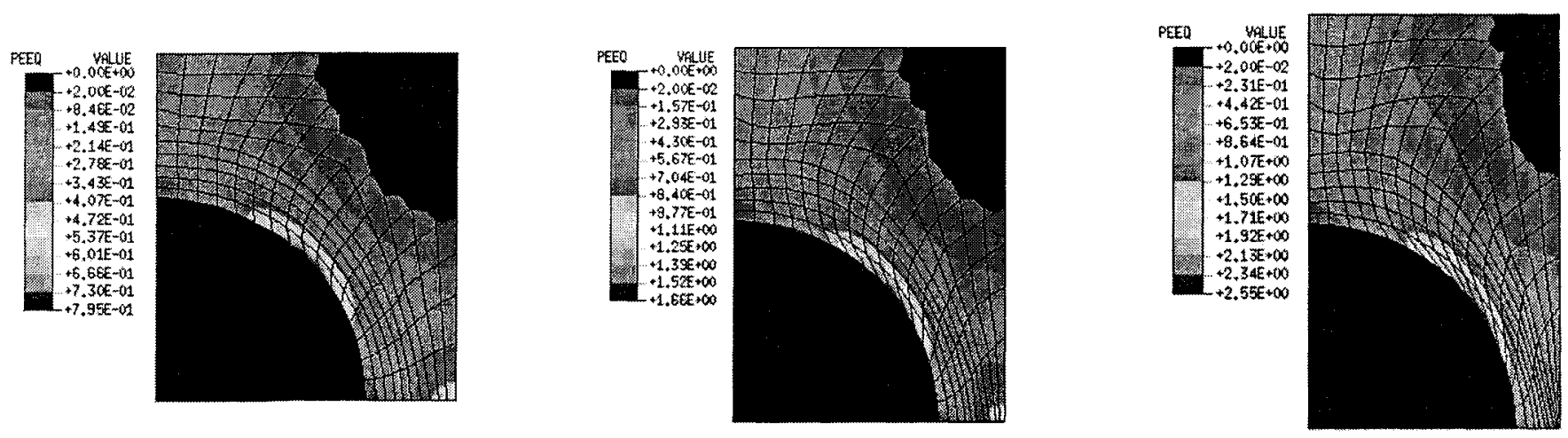

(b)
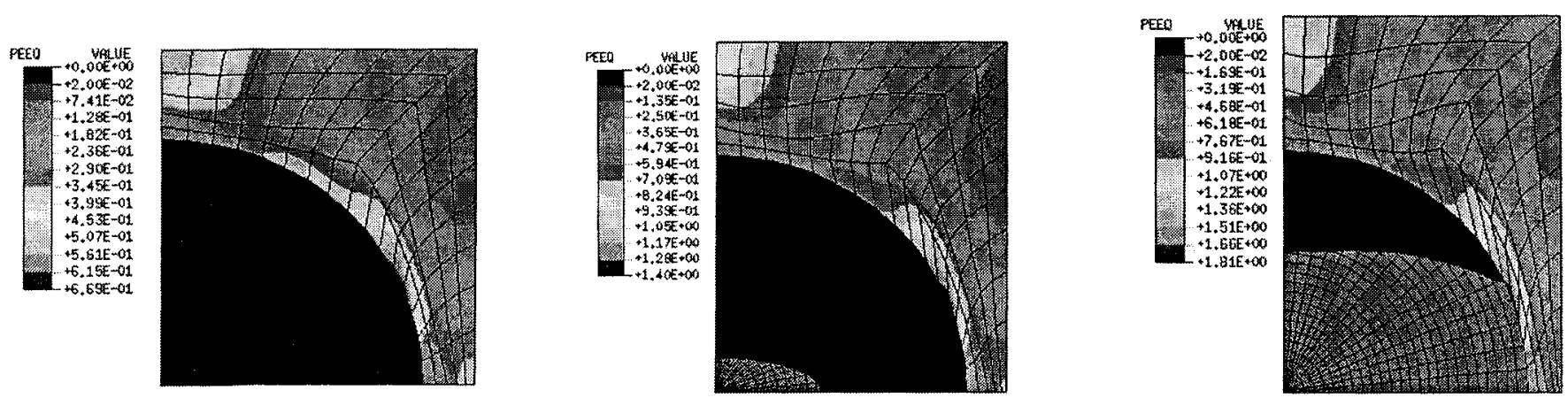

(c)

Fig. 5.10: Contours of effective plastic strain for nominal strains of $10 \%, 20 \%$, and $30 \%$ and $V_{m} \approx 35 \%$ a) Plane strain (PS2) model b) Plane strain (PS1) model

c) SHA axisymmetric model. 


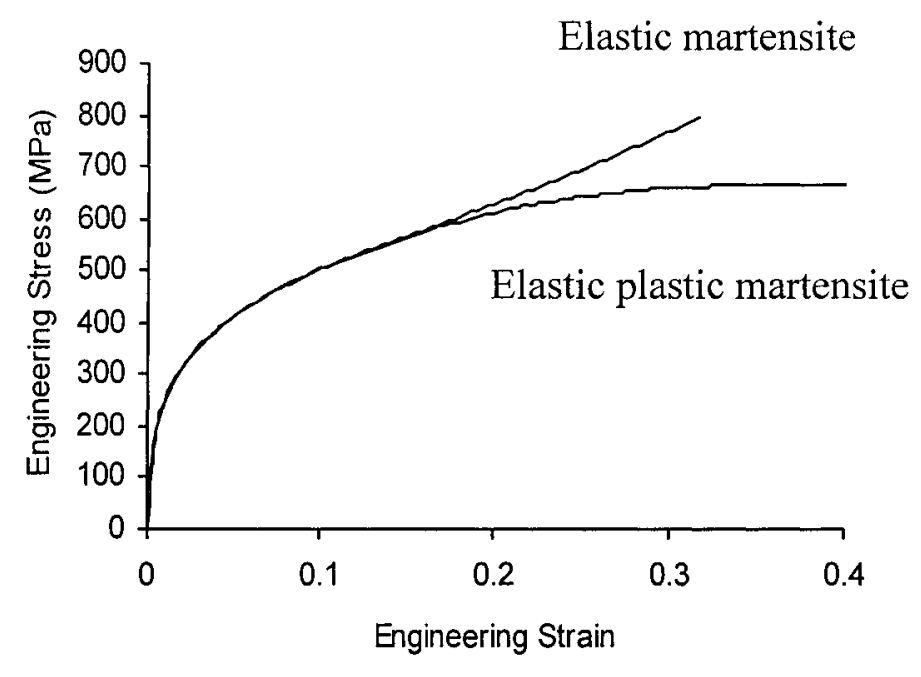

Fig. 5.11: Predicted behavior using SHA axisymmetric model with $V_{m}=\mathbf{2 9 . 4 \%}$ treating martensite as an elastic and an elastic-plastic solid.

\subsection{Discussions and Conclusion:}

Among the models investigated, only the axisymmetric SHA model displays the trend of increase in the engineering stress and the reduction of the onset of strain localization with increasing $V_{m}$. In both PS1 and PS2 cases, the strain hardening in the material was overpredicted, which unrealistically suppressed localization as shown in Fig. 5.5 b\& c.

The above phenomenon is attributed, most significantly, to the deformation stages, which take place in the martensite particles and the ferrite matrix. As mentioned earlier, Shen et al. (1986) and Rashid and Cprek (1978) have shown experimentally, using scanning electron microscopic micrographs, that the martensite particles deform after the ferrite matrix is excessively deformed. Shen et al. (1986) have also shown using a scanning electron microscopy equipped with a straining stage that at low $V_{m}$ only the ferrite matrix deforms with no measurable strain occurring in the martensite particles. Very small 
strains in the peripheral areas of martensite islands were reported using an interferential microscope. At high $V_{m}$ they have shown that shearing of the interface between martensite and ferrite occurs extending the strains into the martensite islands.

All the models considered have similar stress-strain trends at low $V_{m}$ as depicted in Fig. 5.6. The martensite particles undergo no measurable strain at low $V_{m}$, which is in agreement with experimental observation by Shen et al. (1986). At high $V_{m}$, however, the martensite particles in PS1 and PS2 models behave elastically while it plastically deforms in the axisymmetric SHA case. Although, the plastic deformation that takes place in the martensite particles is small, it is believed to be an important phenomenon, which alters the mechanics of deformation of the aggregate significantly as shown in Fig. 5.11. The inability of plane strain models to show any plastic deformation in the martensite particles is believed to be the main reason behind the suppression of the localization behavior they display. In the plane strain idealization, the martensite particles are geometrically assumed to be long cylinders in a ferrite matrix, rather than a spherical particle in a cylindrical matrix, which contradicts the real nature of the material geometry. As a result, for the same volume fraction, the area fraction of martensite is larger in the axisymmetric model than the plane strain models, causing plastic yielding to occur at lower strains, which more closely resembles the actual material response. This increased area or increased particle dimension, results in more excessive strain in the ferrite matrix similar to what Shen et al. (1986) have reported experimentally, introducing more localized strain in the martensite particles through the interface especially at the adjacent interface where excessive ferrite straining exists, and hence causes it to plastically deform.

Shen et al. (1986) reported the extent to which the martensite and ferrite particles deform and show that, in addition to strain being inhomogeneous in both the ferrite and the martensite, strains in both increase linearly with increasing the macroscopic strain of the specimen. They have also reported that the martensite plastic deformation is negligible at nominal strain levels of about $10 \%$ and in the range of $5 \%-10 \%$ at $30 \%$ nominal strain of the specimen, which agrees well with results observed in the axisymmetric SHA model. It 
is worthwhile mentioning that Shen et al. (1986) reported martensite deformation levels for various combinations of $V_{m}$ and $\% \mathrm{C}$ in the steel. Increasing the $V_{m}$ with constant $\% \mathrm{C}$ in the steel would cause dilution of the carbon in the martensite particles, which reduces its strength, but this is reported to be significant only in volume fractions above $30 \%$. Dilution effect in the levels of $V_{m}$ reported by Shen et al. (1986) are negligible as the plastic deformation of the martensite particles are reported to be very close in all the cases.

From the above discussion it can easily be seen that the axisymmetric model possesses the intrinsic ability to predict the experimentally reported DP-steels responses in general at different stages of $V_{m}$ while the plane strain models fail to predict the material response at intermediate and high $V_{m}$. Therefore, plane strain models will be ignored in any further investigation as they fail to address the essential mechanisms and mechanics of the deformation of the DP-steels. In the next Chapter the axisymmetric SHA model will be investigated further to investigate the effect of martensite particle distribution for which the plane strain (PS2) was developed as this effect has been reportedly stressed upon in the literature by some workers for materials other than DP-steels. Obviously, since the PS2 model was shown to be incapable of describing the material behavior, the axisymmetric SHA model will be used with certain modifications to address the issue of particle interaction as will be seen in the next Chapter. 


\section{CHAPTER}

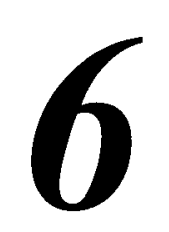

\section{NUMERICAL MODELING OF PARTICLE SIZE DISTRIBUTION IN DP-STEELS}

DP-steel material behavior is best represented by the axisymmetric SHA model compared to the other plane strain models as elucidated in the previous Chapter. This result is utilized further in this Chapter to capture an important aspect reportedly stressed upon in the literature, which is the particle size distribution effect. In this Chapter the particle size distribution is investigated by introducing two particles in the model to visualize its effect on the mechanics and mechanisms of deformation of the DP-steels. This effect, as will be shown shortly, has significant influence on the behavior of DP-steels especially at intermediate and high $V_{m}$. 


\subsection{The Effect of Particle Size Distribution on Material Behavior:}

In order for a certain model to capture the correct material behavior, it must capture the mechanics and mechanisms of deformation the material displays as determined from experimental observation. Some aspects of the deformation process were captured by the SHA model introduced in the previous Chapter which made it the only candidate for further investigation of other important features in the deformation process of DP-steels. Particle size distribution has been reported by some workers (e.g.: Socrate et al. (2000) and Tvergaard (1996)) to have significant influence on the deformation process in a void containing material. Although the behavior of voids is quite different than that of elasticplastic particles, the effect of a distribution of sizes raises interesting possibilities for the micro mechanical modeling of DP-steels. In real void-containing materials, there is a distribution of void sizes resulting from different sizes of inclusions at which voids nucleate. In a study of the micro mechanics of coalescence, Faleskog and Shih (1996) introduced a representative material volume containing several large voids and a population of micro voids present from the beginning using a plane strain model. They modeled all voids as discrete entities and have shown that a local zone of high stress concentration emanates from the large void and spreads across the material raising the stresses at the nearby micro voids. As a result, the micro voids grew unstably. Tvergaard (1996) investigated the effect of void size difference on growth and cavitation instabilities. His analysis was based on an axisymmetric unit cell model, which allows for the representation of a number of spherical voids. Tvergaard has shown that for a range of rather low stress triaxiality, the relative growth rates of the two voids vary with initial void volume fraction and for very high stress triaxialities interaction effects become important if the initial void volume fraction is sufficiently low and predicted that only one of the voids grew large. In another work, Tvergaard (1998) investigated the interaction of very small voids with large voids to determine whether or not local stress increases induced by the large void result in cavitation instability at the tiny void. He has shown that for overall stress levels as large as those reached ahead of a blunting crack tip, 
cavitation instability does not form at the small voids due to the interaction with the large void but the results show that localization of plastic flow in the unit cell plays an important role. Orsini and Zikry (2001) in a study of void growth and interaction in crystalline materials have used a micro mechanical model with discrete voids at seven different positions to study the interrelated physical mechanisms that can result in ductile fractures. They used a rate dependent constitutive formulation and allowed the plane strain micro model to neck by not restricting the side of the model to be straight. Although the ligament between the voids necks in the micro level, this cannot be a realistic representation of the material behavior since continuity would not be preserved. Becker et al. (1989) in a study of the effect of void shape on void growth and ductility in axisymmetric tension tests reported bimodal $\mathrm{MnS}$ distribution in the matrix material. This was determined using automatic image analysis as the inclusions were judged to be the void size distribution because they were reported to be soft and broke up during the early stages of tension tests. Although they have shown the size distribution in their material, they did not consider it but focused on void shape effect.

As in a material containing voids, the assumption of different particle sizes in a heterogeneous material is more realistic than one with a single particle size. Socrate et al. (2000) in a study of multiple crazing in high impact, toughened, polystyrene have reported that particle diameters in such materials are in the range 1-4 $\mu \mathrm{m}$ with an average size around $2 \mu \mathrm{m}$. The particles smaller than $0.8 \mu \mathrm{m}$, which corresponds to $1 / 4$ the particle size, do not initiate crazes, unlike larger particles. They have also reported that the most effective particle size for toughening is in the range $1-2 \mu \mathrm{m}$, which is between the average and one-half the average particle size. In addition, Goods and Brown (1979) (also presented in Chapter 2) presented a dislocation model, which describes the nucleation and growth of voids, based on particle sizes. It is evident in that model that as the particle size decreases the local stress requirement for decohesion increases and thus void nucleation will be more difficult to form with the small particles than with larger ones. This is due to the lower stress the smaller particles induce compared to the higher 
stress bigger particles initiate in the surrounding area around the inclusions. The above clearly indicates that the particle size has significant influence on the material behavior.

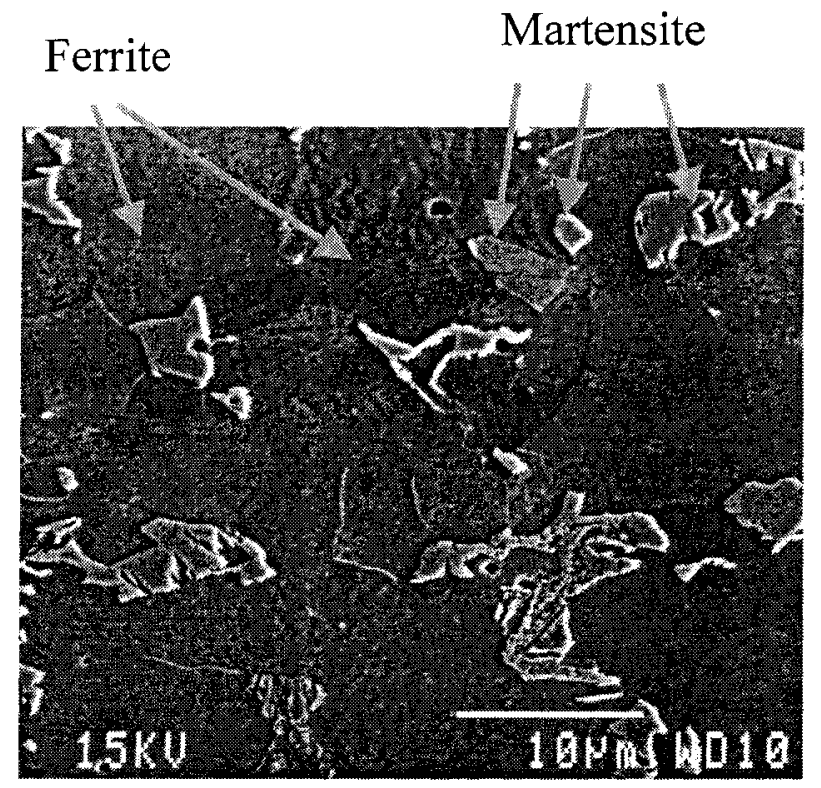

Fig. 6.1: Typical microstructure of dual-phase steel showing the variation of the particle sizes.

In typical DP-steels the variation of the particle size distribution is observed as can be seen in a typical micrograph shown in Fig. 6.1. In addition, based on observations Shen et al. (1986) have reported, the different martensite particles with different sizes have experienced different stages and deformation mechanisms. They reported that the large martensite particles experience plastic deformation after necking while the small particles remain elastic in agreement with observations made by Rashid and Cprek (1978).

The above evidence and observations led the author to investigate the effect of the particle size distribution, which more realistically represents the reality of the DP-steel microstructure. In this Chapter, a micro mechanical model for the DP-steels, which allows for the investigation of the effect of particle size difference, is developed. The 
point of interest is whether or not the size difference in DP-steels has any effect on the predicted response of such materials.

\subsection{Finite Element Modeling:}

The three main aspects of the micromechanical modeling technique, namely, the RVE, constitutive behavior of each phase and the homogenization method will be the same as the ones used in the last Chapter and thus will not be repeated here. The exception is that, in this Chapter, the idealization used in this model (RVE), shown in Fig. 6.2, has periodic hard particles of two sizes dispersed in the softer matrix. Due to symmetry only the dashed area is modeled. The small and large particles are chosen to be staggered with respect to each other to keep the material homogeneous.

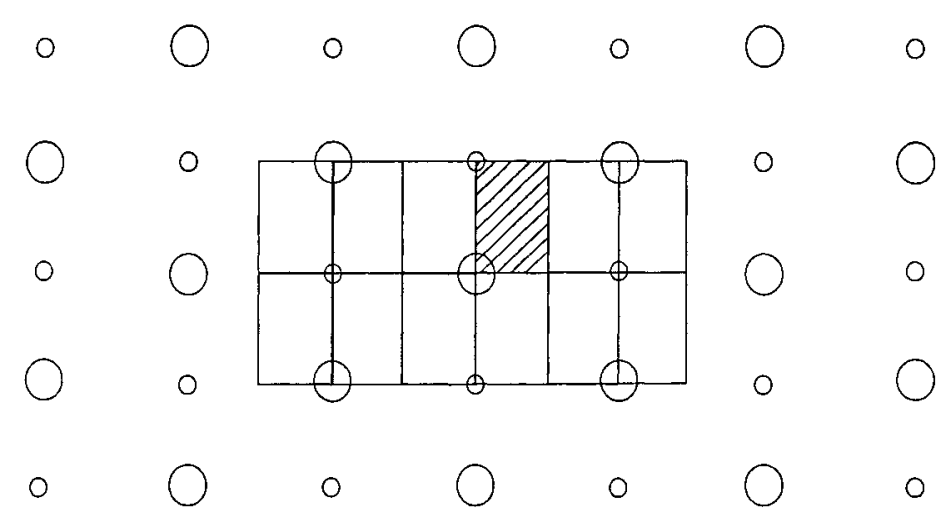

Fig. 6.2: Axisymmetric two-particle model RVE idealization, showing the homogeneous dispersion of small and large particles.

In the analysis carried out in this work, two meshes are considered. One has a single martensite particle and equal side dimensions (mesh1) and the other one (mesh2) has two martensite particles and unequal side dimensions $(H=2 \mathrm{~L})$, which facilitate the investigation into the effect of size difference on the mechanical properties of DP-steels. 
Both meshes are illustrated in Fig. 6.3. Mesh2 is made of two identical upper and lower parts. The mesh around each hard particle is chosen to be identical to avoid any effect of meshing on the analysis. Referring to Fig. $6.3 \mathrm{~b}$, the volume fraction is computed as $\frac{2\left(a^{3}+b^{3}\right)}{3\left(L^{2} H\right)}$. Symmetry boundary conditions are used for sides S1 and S2, while side S3 has a uniform displacement in the $x_{1}$ direction and side S4 has uniform displacement in $x_{2}$ direction.

The aggregate strains are computed as

$$
\begin{aligned}
& E_{11}=\ln \left(u_{1}\left(L, x_{2}\right) / L\right) \\
& E_{22}=\ln \left(u_{2}\left(x_{1}, H\right) / H\right)
\end{aligned}
$$

The engineering normal stress in the $x_{2}$ direction is computed in the same way as presented in Chapter 5.

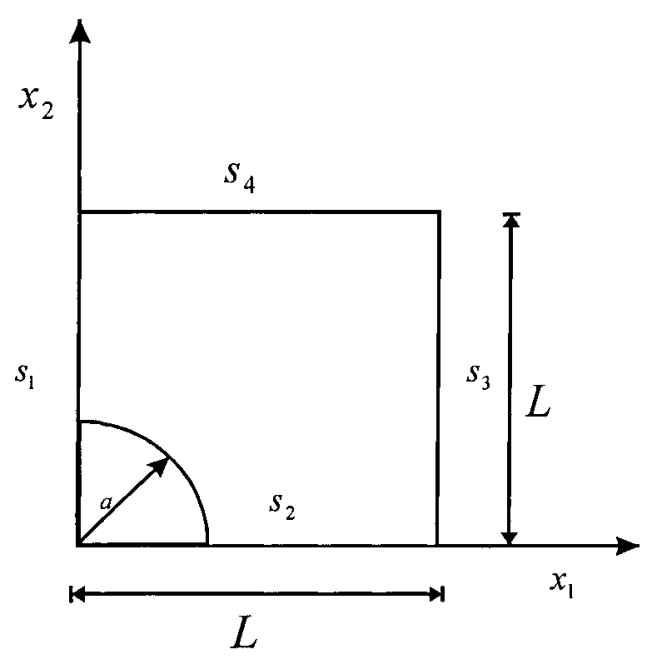

(a)

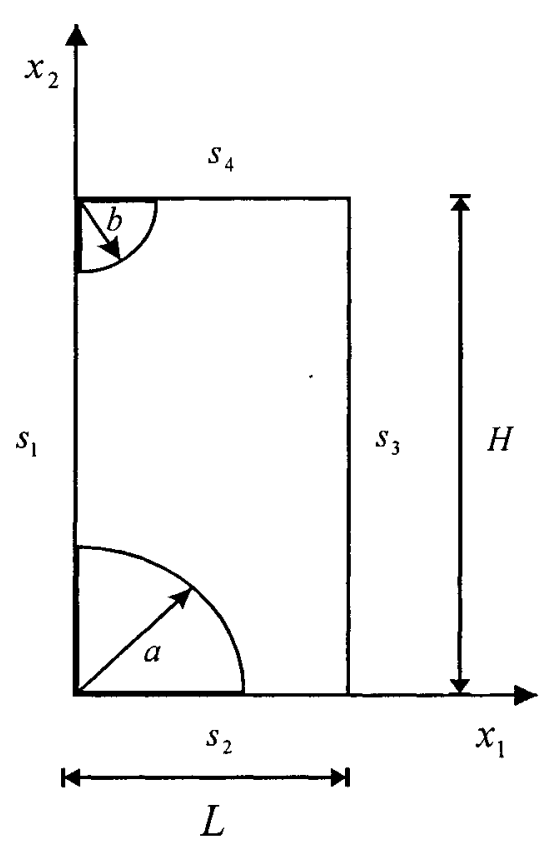

(b)

Fig. 6.3: Dimensions of the micro mechanical models a) single particle model b) two particle model. 
The evolution of the plastic strain and stress-strain response for mesh1 and mesh2 with equal particle sizes $(\mathrm{a}=\mathrm{b} ; \mathrm{R}=\mathrm{b} / \mathrm{a}=1)$ at the same $V_{m}$ are identical. Mesh2 displays the expected obvious symmetry when the two particles are of equal size as depicted in the equivalent plastic strain evolution shown in Fig. 6.4 for $V_{m}=13 \%$.

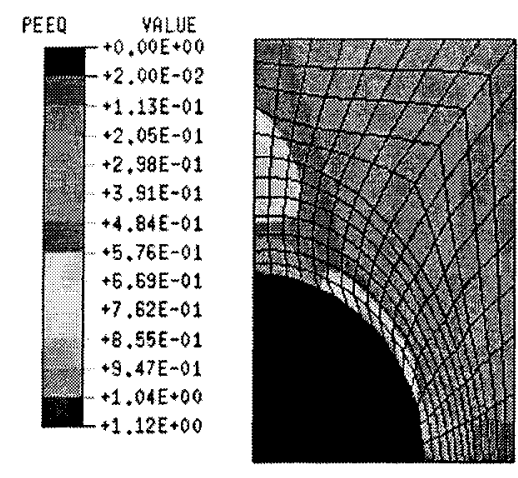

(a)

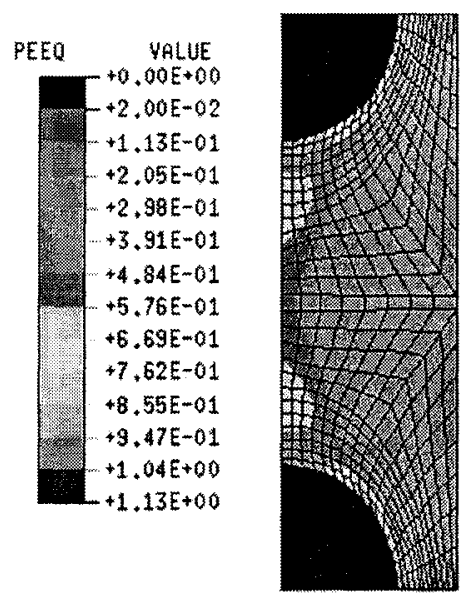

(b)

\section{Fig. 6.4: The evolution of the equivalent plastic strain for the single particle model and the two particle model at particle size ratio, $\mathrm{R}=1$ and $V_{m}=13 \%$.}

The response of the aggregate at different particle size ratios $(\mathrm{R}=\mathrm{b} / \mathrm{a})$ at $V_{m}=6.8 \%, V_{m}=$ $17 \%$ and $V_{m}=21.5 \%$ is shown in Fig. $6.5 \mathrm{a}, \mathrm{b}$ and $\mathrm{c}$ respectively. It can clearly be seen that the contribution of the particle size on the predicted response of the model is very small at low $V_{m}$, while it is considerably more important at intermediate and high $V_{m}$. The plot of the predicted tensile strength, vs. $\mathrm{R}$ is shown in Fig. 6.6 for the case of $V_{m}=17 \%$. The figure shows that the strength of the aggregate increases from $R=1$ to $R=3 / 4$ and reaches a maximum value at $R=1 / 2$. Reducing the value of $R$ further reduces the strength but the reduction is quite small. The other cases $\left(V_{m}=6.8 \%\right.$ and $\left.V_{m}=21.5 \%\right)$ follow the same trend, but with different values for the ultimate strength. 
For low values of $\mathrm{R}$ (e.g. $\mathrm{R}=1 / 16$ ) the predicted responses were expected to decline to values similar to the response of the model at $\mathrm{R}=1$ since the smaller particle becomes negligible. However, this did not occur as depicted in Fig. 6.5 and Fig. 6.6, which led the author to investigate the effect of the dimensions $\mathrm{H}$ and $\mathrm{L}$ on the response of the model with unequal parts. The predicted responses of the models at $R=1 / 16$ and $R=0$ were compared and found to be identical but different from results using mesh 1 for the same volume fraction, which clearly indicates that the dimensions of the cell $(\mathrm{H}$ and $\mathrm{L})$ have an influence on the response of the model. Similar effects have been observed by Pardoen and Hutchinson (2001) in a study of a model for void growth and coalescence. 


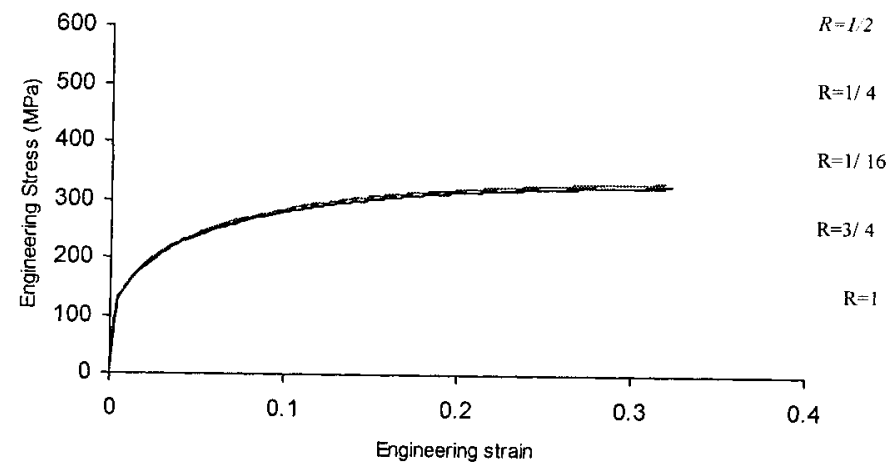

(a)

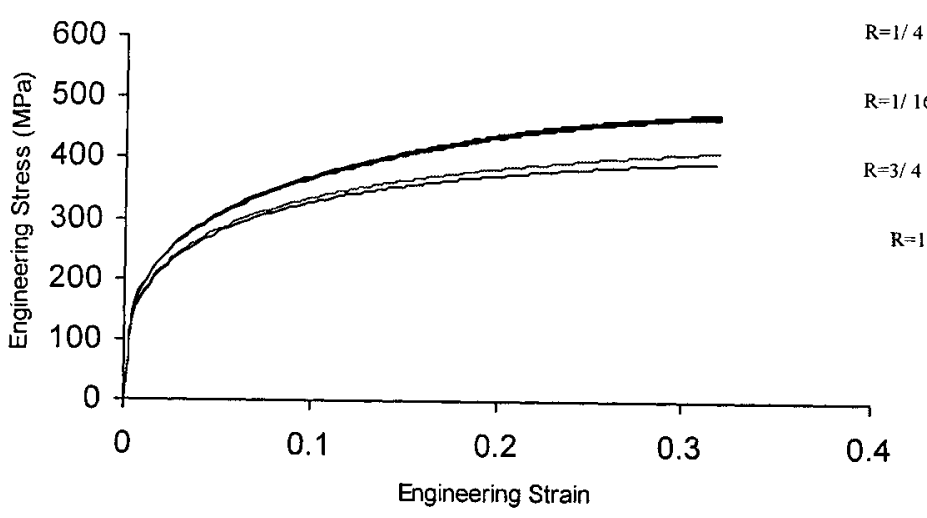

(b)

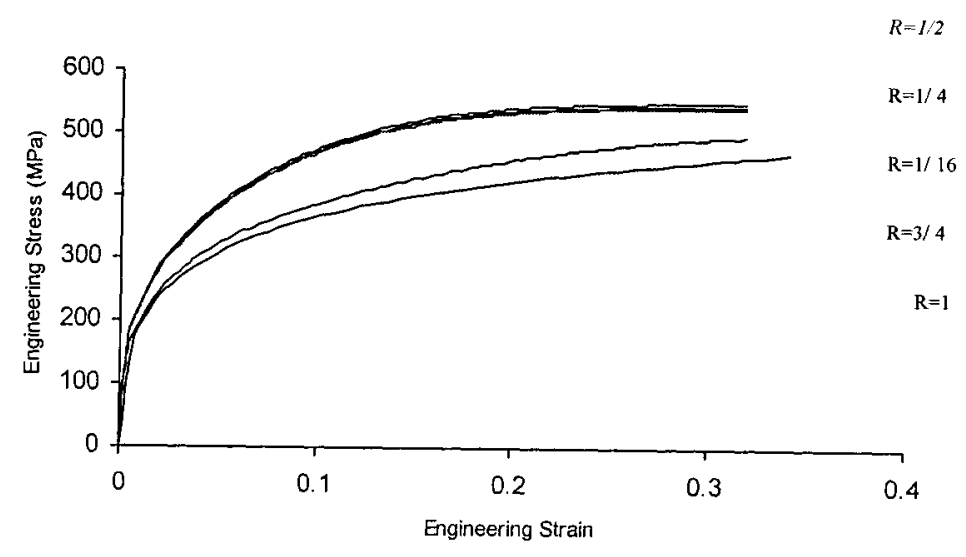

(c)

Fig. 6.5: The predicted response of the two particle model at three different volume fractions of the harder phase a) $6.8 \%$ b) $17 \%$ c) $21.5 \%$ for particle size ratios of

$$
\mathrm{R}=1, \mathrm{R}=3 / 4, \mathrm{R}=1 / 2, \mathrm{R}=1 / 3 \text { and } \mathrm{R}=1 / 16 \text {. }
$$




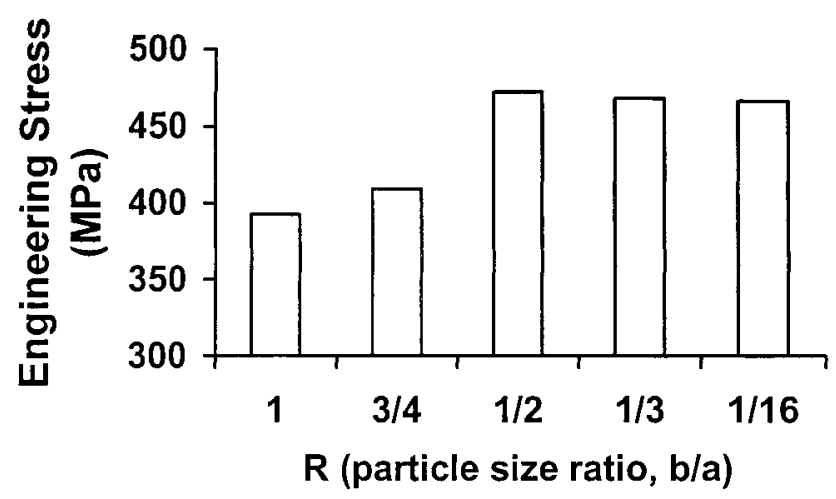

Fig. 6.6: The predicted tensile strength of the aggregate at the particle size ratios, $R$ $=1, R=3 / 4, R=1 / 2, R=1 / 3$ and $R=1 / 16$ for $V_{m}=17 \%$.

The effect that particle size distribution has on the response of DP-steels can also be seen by examination of contours of equivalent plastic strain. Figure 6.7 shows the distribution of plastic strain for several cases of combinations of volume fraction and $R$ value. At low $V_{m}$ (Fig. 6.7 a) the martensite particles do not plastically deform, which is consistent with results found for $\mathrm{R}=1$, and also explains the negligible effect of particle size distribution seen in the stress vs. strain response of Fig. 6.5 a. However, at higher volume fractions of martensite (Fig. 6.7 b), the distribution of plastic strain in the martensite is heterogeneous, with the large particle exhibiting plastic strain as high as $15 \%$ and the smaller particle remaining elastic. By comparing Fig. $6.7 \mathrm{~b}$ and Fig. $6.7 \mathrm{c}$, it can also be seen that a heterogeneous distribution of particle sizes results in plastic deformation in the martensite occurring at lower volume fractions. These differences are manifested in the differences of the stress vs. strain response apparent at medium and high martensite volume fractions seen in Fig. 6.5. 

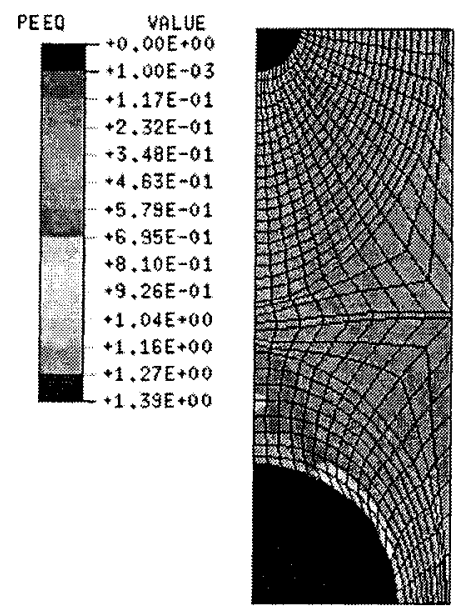

(a)

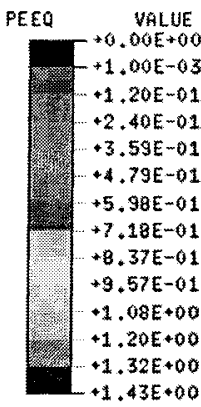

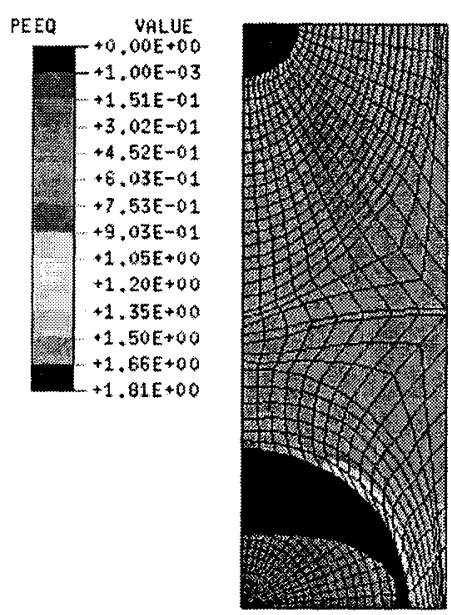

(b)
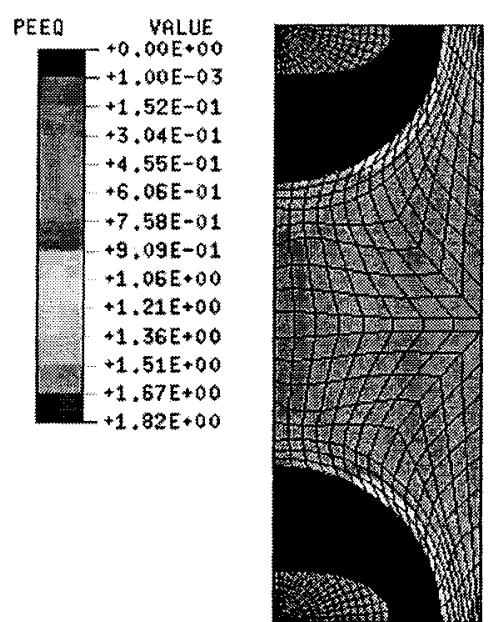

(d)

Fig. 6.7: Contours of equivalent plastic strain for nominal strain of $30 \%$, a) $\mathrm{R}=1 / 4$, $V_{m}=9 \%$ b) $\mathrm{R}=1 / 4, V_{m}=13 \%$ c) $\mathrm{R}=1, V_{m}=17 \%$ and d) $\mathrm{R}=1, V_{m}=22 \%$.

Quantitative comparison of the results predicted by the model to experimental data reported in the literature are difficult to perform because even small differences in chemistry or grain size between the materials used for the constituent properties here and that used in available experiments on DP-steel will have significant influence on the behavior. Nevertheless, certain aspects of the behavior of DP-steels, which is attributed to their inherent nature can be examined. One of these characteristics is the decrease in uniform strain observed with increasing volume fraction of martensite (Rashid and Cprek 
(1978)), which is an important trade-off to obtain increased strength. Experimental results of uniform strain as a function of volume fraction of martensite from Davies (1978) is shown in Fig. 6.8 along with predicted results using three different modeling assumptions. Clearly, only the model containing the heterogeneous particle size distribution captures the same trend as the experimental results, although quantitative differences are noted.

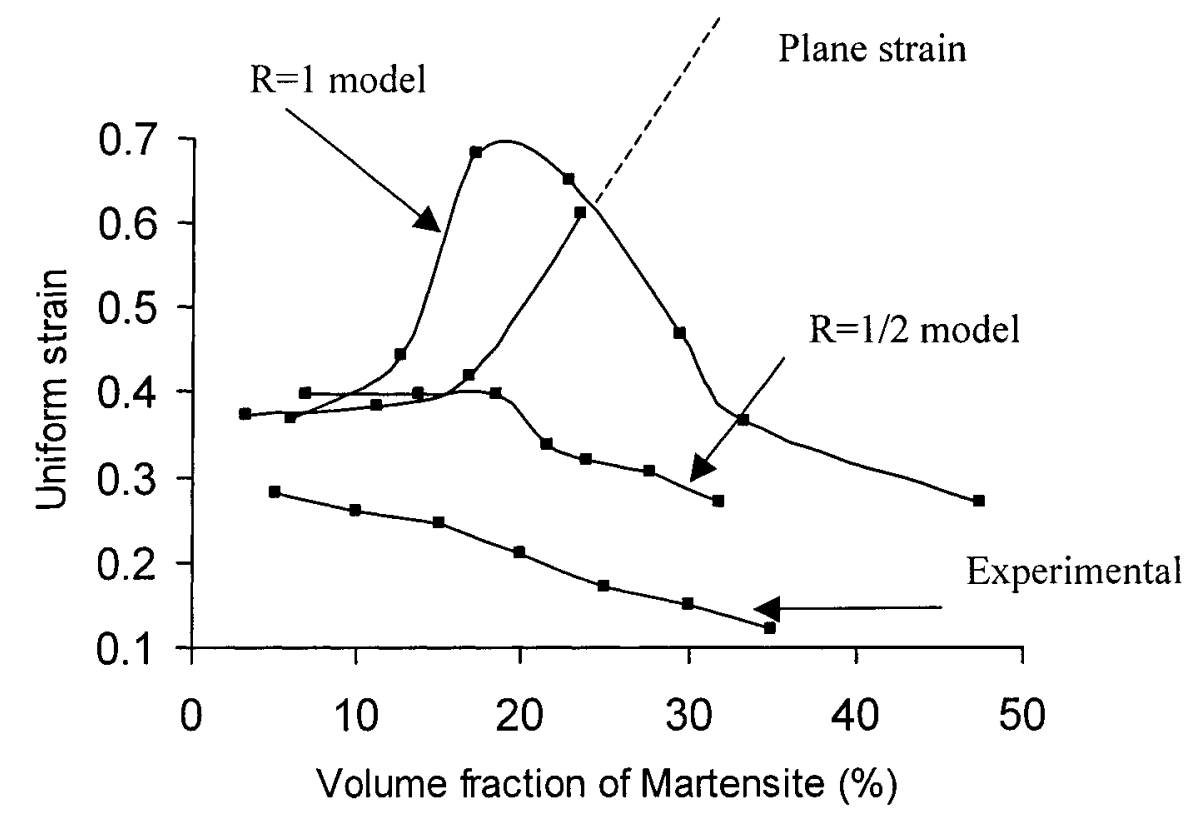

Fig. 6.8: Experimental results (Davies (1978)) of uniform strain vs. volume fraction of martensite compared to results using three modeling assumptions.

Another characteristic of DP-steels attributed to their heterogeneous nature is their high UTS to yield ratio or high strain hardening rate, which is particularly attractive for many forming operations. Figure 6.9 shows the strain-hardening rate vs. engineering strain using data from Shen et al. (1986) at two martensite volume fractions compared to results from different modeling assumptions. At $V_{m}=10 \%-13.8 \%\left(\right.$ low $\left.V_{m}\right)$ the closest trend is 


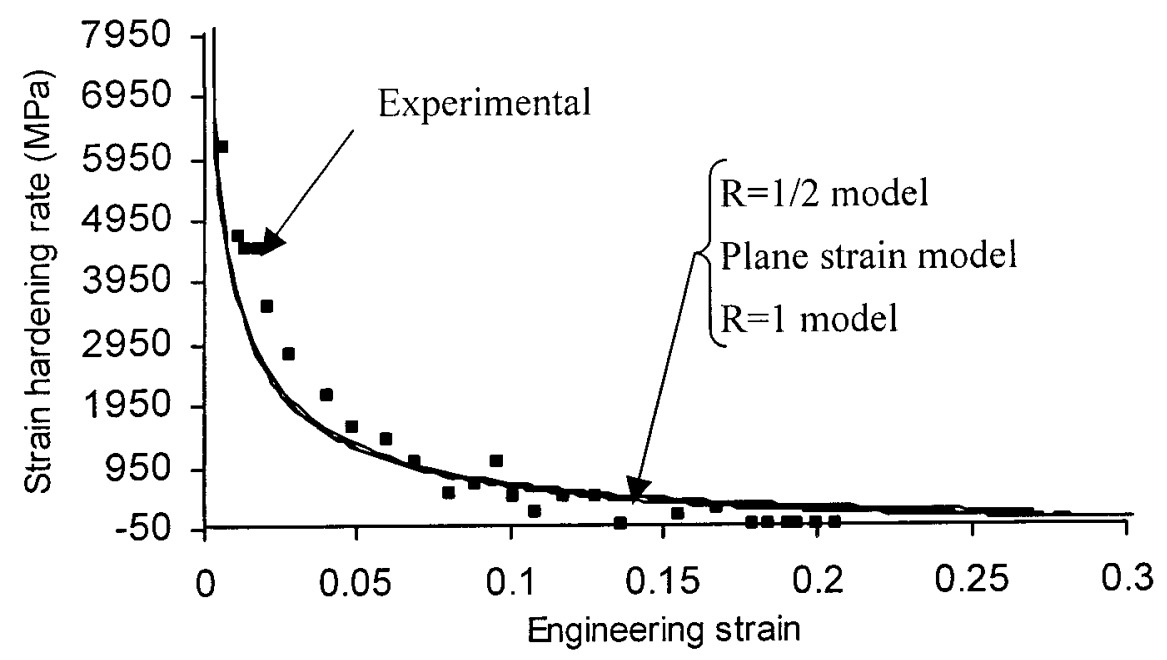

(a)

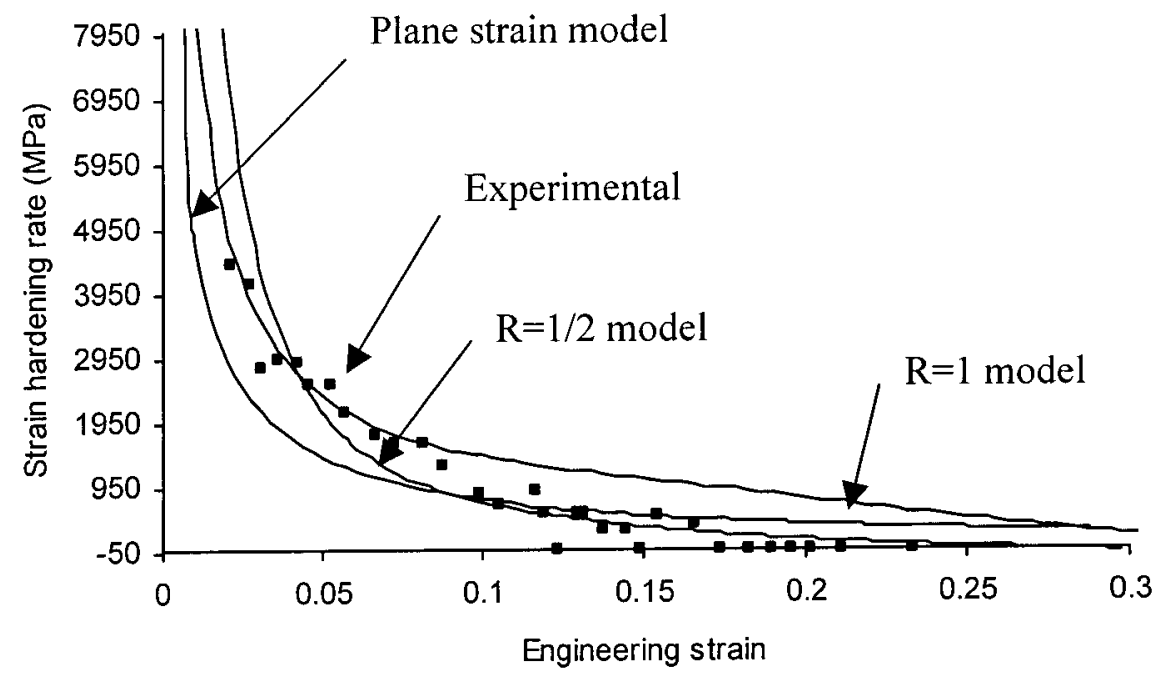

(b)

Fig. 6.9: Experimental results of strain hardening rate from Shen et al. (1986) compared to three modeling assumptions a) low $V_{\mathrm{m}}$ b) high $V_{\mathrm{m}}$. 
displayed by the model with $\mathrm{R}=1 / 2$ even though the difference is not significant. At $V_{m}=$ $30 \%-33 \%$ (high $V_{m}$ ) the model with $\mathrm{R}=1$ shows better agreement with the experiment up to $3 \%$ strain but from $4 \%$ strain onwards the model with $R=1 / 2$ shows close agreement with the experimental result while the other two models deviate significantly from the experimental results. The comparisons in Fig. 6.8 and Fig. 6.9 clearly show that the model with $\mathrm{R}=1 / 2$ is able to better capture the strain hardening rate from the early stages of straining to the onset of instability than the model with $\mathrm{R}=1$ and the plane strain model.

\subsection{Discussions and Conclusion:}

Different particle size ratios have been examined to investigate the size ratio effect on the micro mechanical model to evaluate its importance in prediction of the response of the material. The ratios considered were $\mathrm{R}=1, \mathrm{R}=3 / 4, \mathrm{R}=1 / 2, \mathrm{R}=1 / 4, \mathrm{R}=1 / 16$ and $\mathrm{R}=$ 0 . As mentioned earlier, $R=1$ presents the symmetric case where the results are identical to the single particle case as in mesh1 and thus no effect of particle size interaction is noticed. As one of the particles is reduced in size at a constant volume fraction of martensite the effect of the difference in particle size becomes more noticeable and reaches its maximum effect at particle size ratio $R=1 / 2$. Reducing the smaller particle size further to $R=1 / 4$ and $R=1 / 16$ was physically expected to result in the decline of the stress-strain curve to reach $\mathrm{R}=1$ response due to the little effect of the very small particle. This trend was noticed but with a very limited decrease in stress values, a fact which is attributed to the difference in cell dimensions, as noted previously. Changing the RVE cell geometry ratio will bring in the effect of particle distribution in the matrix, which was not covered in this work, but has the same effect on the cell model when the cell dimension $\mathrm{L}$ is reduced and $\mathrm{H}$ is kept constant.

Further examination of the results show that at low $V_{m}$ and particle size difference of $\mathrm{R}=$ $1 / 2$ there is no measurable difference in the response compared to the model with $R=1$. 
At high $V_{m}$ the effect is clear as shown in Fig. 6.5. In addition, the difference comes about only when the larger martensite particle undergoes plastic deformation and this occurs at the larger particle only as shown in Fig. 6.7 b. The onset of plastic deformation of the larger martensite particle in the $R=1 / 2$ model occurs at lower volume fractions of martensite than the particles in the $\mathrm{R}=1$ model. This takes place due to the fact that the ferrite phase surrounding the larger particle is very constrained compared to the ferrite in the latter case at the same $V_{m}$, which forces the larger martensite particle to undergo earlier plastic deformation. It is worthwhile mentioning that Shen et al. (1986) reported martensite deformation levels for various combinations of $V_{m}$ and $\% \mathrm{C}$ in the steel. Increasing the $V_{m}$ with constant $\% \mathrm{C}$ in the steel causes dilution of the carbon in the martensite particles, which reduces its strength, but this is reported to be significant only in volume fractions above $30 \%$. Since the purpose of this section is to investigate the effect of particle size distribution on the mechanical behavior of DP-steels, this effect is not considered, although it is believed to have an influence on the overall behavior of the material.

From the analysis above it is noticed that only the larger particle experiences plastic deformation while the smaller particle undergoes no measurable plastic deformation. The difference in the strain distribution among the martensite particles can be attributed to the size effect since the plastic deformation in the martensite particles takes place due to the strain or load being transferred from the ferrite matrix to the martensite particles. Since the small particles with small interface surface would take less strain than the larger particle sizes, the larger particles deform faster than the smaller ones and the small ones do not experience any plastic deformation.

In agreement with the above experimental observations, the model with two particle sizes shows two different mechanisms of deformation occurring in the martensite particles. The larger particle neighboring the highly strained ferrite matrix, which Shen et al. (1986) considered as weak martensite particles, undergo plastic deformation at intermediate and high $V_{m}$, while the smaller particle experiences no plastic deformation. In other words, 
the larger particle shows a duplex deformation mechanism while the smaller martensite particle shows only a particle strengthening mechanism.

In this Chapter a micro mechanical model for the DP-steels consisting of martensite particles of two different sizes dispersed in a ferrite matrix has been developed. The model captures the effect of the realistic assumption of different particle sizes being present and distinguishes two mechanisms of deformations taking place side by side in the process of tensile straining. Due to the complex nature of strain hardening and due to different mechanisms being present in the deformation process, previous authors failed to develop a mathematical model that can completely describe the deformation process in DP-steels. In addition to being successful in capturing the trend of increasing strength and decreasing uniform strain with increasing volume fraction of martensite which was also shown in the previous Chapter, this model can capture the effect of different particle sizes and predict the existence of two mechanisms of deformation during the deformation process. In doing so, the model also captures the onset of plastic instability implied by the uniform strain and the interesting steep increase in the strain hardening rate, which distinguishes DP-steels from other types of steels.

In addition to the particle size effect, there are other important material features that need to be investigated. In the next Chapter different DP-steel material considerations will be examined and ways to implement them in this developed model will be discussed in detail before comparison between experimental findings laid down in Chapter 4 and modeling predictions which will be presented in Chapter 7 are made. 


\section{CHAPTER}

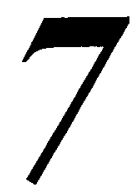

\section{DUAL PHASE STEEL MATERIAL MODELING AND EXPERIMENTAL VALIDATION}

In this Chapter DP-steel material behavior considerations will be discussed and implemented in the two-particle model developed in Chapter 6. The most important material considerations are the ferrite grain size effect, martensite softening by carbon dilution and the particle size effect discussed earlier. These effects together with the required metallurgical measurements and calculations will be presented in detail in this Chapter. Comparison between experimental observations and the predicted responses for the material used in the experimental work (presented in Chapter 4) will be conducted to display the capability of the current model to capture the DP-steel material behavior at different $V_{\mathrm{m}}$ with the aforementioned material considerations being implemented into the model. 


\subsection{Material Considerations:}

There are several material considerations that were judged to be the most important in modeling the DP-steel material behavior on the phase level; this judgment was based on preliminary investigation conducted using the constitutive behavior from the literature, which have been shown and utilized in Chapter 5 and 6, to see their effect on the mechanical behavior of the DP-steel. In the subsequent sections, these material effects are implemented in the two-particle model with the constitutive material behavior for the constituents produced in this work. The material considerations are discussed below:

\subsubsection{Martensite Softening:}

Martensite softening by carbon dilution, which is well known, is taken into consideration in modeling the plastic behavior of the martensite and found to be of great importance, even though the extent of plastic deformation in the martensite is quantitatively small as shown in Chapter 5. The softening of the martensite occurs when its $V_{\mathrm{m}}$ is increased keeping the carbon content in the material constant. Martensite strength characteristics are possessed by the phenomenon of carbon atoms being trapped inside the iron unit cell or crystal structure by fast cooling or quenching which changes the structure of the austenite phase, $\mathrm{FCC}$, to martensite, BCT, and introduces the strength it displays. When the $V_{\mathrm{m}}$ is increased at a constant carbon content, the amount of the carbon content trapped inside the lattice of the iron atoms would not be enough to produce the same strengthening effect they introduce at low $V_{\mathrm{m}}$ and this causes the strength of the martensite to decline as the $V_{\mathrm{m}}$ is increased which is referred to as martensite softening by carbon dilution.

Martensite strength is carbon content dependent only, and in the range of carbon content considered in this work $(0.09 \%)$, it is given by the linear relationship represented by equation 7.1, which is reported to agree well with experimental observations (Speich and Warlimont (1968) and Leslie (1981)). 
Where $\mathrm{C}$ is the \% carbon content by weight in the martensite phase. The carbon content for the various $V_{\mathrm{m}}$ is estimated using the lever rule from the standard $\mathrm{Fe}-\mathrm{C}$ phase diagram (Askeland (1985)) and the results for the carbon content of interest is shown in Fig. 7.1 below. This relation is required in order to relate the martensite strength to $V_{\mathrm{m}}$ instead of carbon content which makes it easier to accommodate the strength change in the model as the dilution effect takes place.

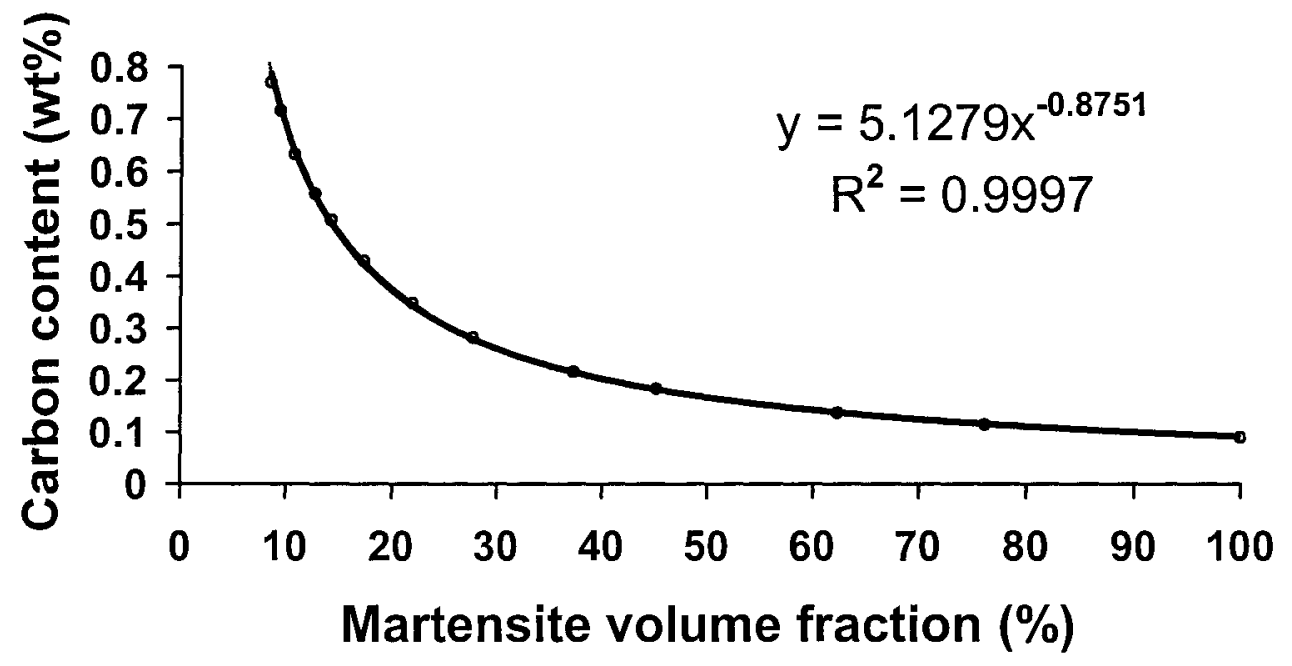

Fig. 7.1: Carbon content for various $V_{m}$ extracted from a standard Fe-C phase diagram using the lever rule.

The effect of martensite softening was investigated numerically to quantitatively appreciate its importance. This investigation is carried out using the single particle model (or $\mathrm{R}=1$ model) as to isolate any other effect from taking place in the model since the $\mathrm{R}$ $=1 / 2$ model would compensate for the particle size distribution, and would not be possible to physically explain the mechanism of deformation involved. The investigation was carried out using Davies (1978) material description for both ferrite and martensite as 
introduced in Chapter 5, one time with implementing the carbon dilution effect and a second time without applying the carbon dilution effect to be able to compare the difference in the mechanism of deformation for the two cases. This was done for $V_{\mathrm{m}}$ of approximately $12.9 \%, 20.1 \%, 31.4 \%, 34.1 \%$ and $55.4 \%$. From these $V_{\mathrm{m}}$ only the ones, which displayed a significant effect on the mechanism of deformation, are shown in Fig. 7.2. The contours of the plastic deformation representing the above cases for a nominal strain of $30 \%$ are shown in Fig. 7.3.

It can be seen from Fig. 7.2, that the difference between the predictions for the two cases, with and without application of carbon dilution effect, are noticeable only above about $V_{\mathrm{m}}$ $=31.4 \%$. The cases for $V_{\mathrm{m}}=12.9 \%$ and $V_{\mathrm{m}}=20.1 \%$ did not show any measurable difference with and without the application of the carbon dilution. This suggests that the carbon dilution effect on the mechanism of behavior DP-steel gets triggered at approximately $V_{\mathrm{m}}=30 \%$. The effect becomes more visible by increasing the $V_{\mathrm{m}}$ as shown in Fig. 7.2 b\&c. The contours of plastic strain for $V_{\mathrm{m}}=20.1 \%$ in Fig. 7.3 a show clearly that there is no measurable difference in the plastic deformation of martensite particle between the two cases. Increasing the $V_{\mathrm{m}}$ shows an increasing effect of the carbon dilution effect as depicted in Fig. $7.3 \mathrm{~b}, \mathrm{c} \& \mathrm{~d}$.

The effect of $V_{m}$ has been investigated by different authors (Jiang et al. (1993), Bag et al. (1999), Tomita (1990), Byun and Kim (1993) and Tomota (1987)). Increasing the volume fraction of the harder phase was found to increase the yield and ultimate strengths of the aggregate. Bag et al. (1999) reported that the increase in strength with $V_{m}$ only extends up to $V_{m} \sim 55 \%$, after which a reduction in strength is observed. The same was observed by Byun and Kim (1993) but at $V_{m}=30 \%$. Byun and Kim (1993) reported using the Joaul-Crussard analysis that the stages of strain hardening display three distinct regions for $V_{m}$ less than $30 \%$ and two stages of strain hardening for $V_{m}$ greater than $30 \%$. Shen et al. (1986) have observed this as well without specifying the value of $V_{m}$ at which this takes place. They attribute this to carbon dilution, which softens the martensite phase, thus dropping the overall strength of the aggregate. 
The predicted results presented above agree well with the observations by Byun and Kim (1993) as they also used the same correlation relating the martensite strength and carbon content which show that the dilution effect becomes practically visible at $V_{\mathrm{m}}=30 \%$. The predictions show that the effect of carbon dilution start to occur at $V_{\mathrm{m}}=30 \%$ with increasing influence as the $V_{\mathrm{m}}$ is increased which agrees with the Byun and Kim (1993) observations quantitatively and the other results qualitatively. It can be easily concluded that carbon dilution effect plays an essential role on the aggregate mechanical behavior of DP-steels and will be considered as one of the material compensations in the present work.

\subsubsection{Ferrite Grain Size:}

The grain size effect on the strength of the steel is also well known and has been reported by Morrison (1966) that the lower yield stress is linearly related to the reciprocal of the square root of the grain diameter as shown in equation 7.2 for $\%$ wt. C content extending from $0.05 \%$ to $0.2 \%$ and for various combinations of alloying elements.

$$
\sigma_{y}=\sigma_{0}+k d^{-\frac{1}{2}}
$$

This relation is utilized to accommodate the grain size effect whenever the grain size effect is seen to be significantly different. The effect of the matrix material strength on the overall material behavior is studied and found to be important.

\subsubsection{Particle Size Distribution:}

This effect has already been discussed in detail in Chapter 6 and found to be significant in capturing the mechanisms and mechanics of behavior of DP-steel material behavior and will not be elaborated further here. 


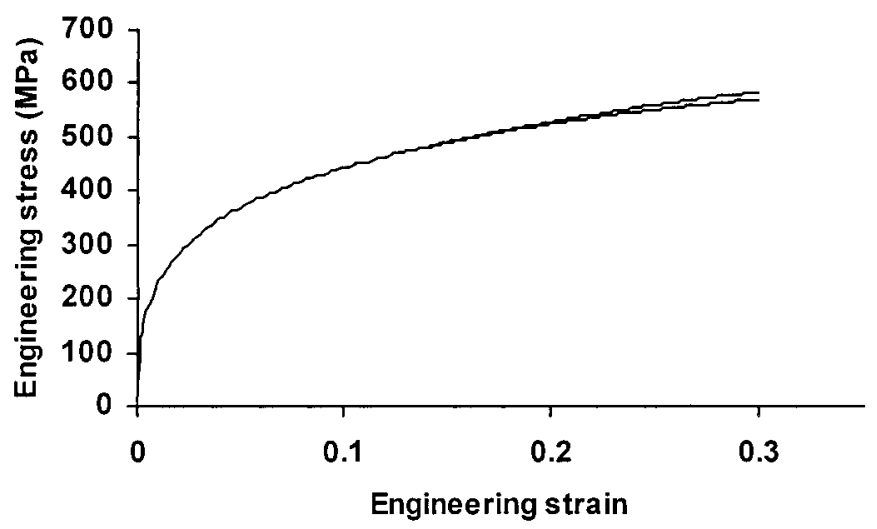

(a)

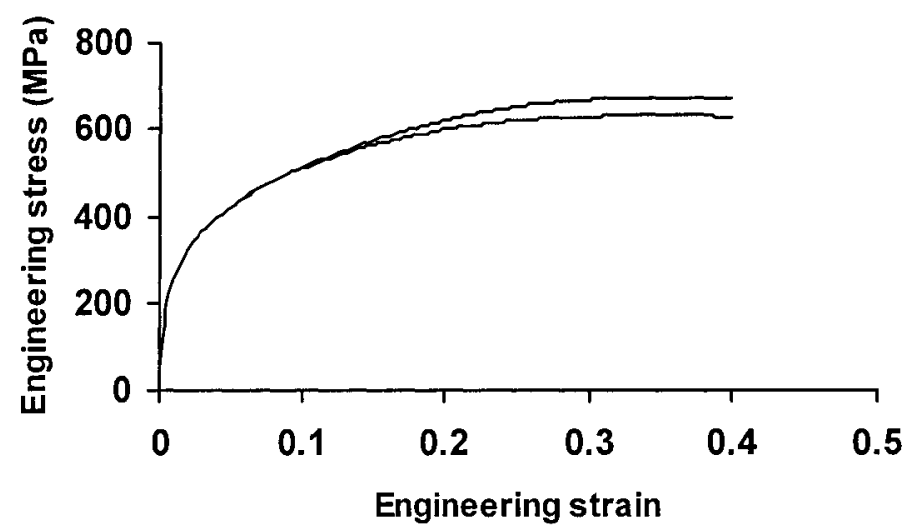

(b)

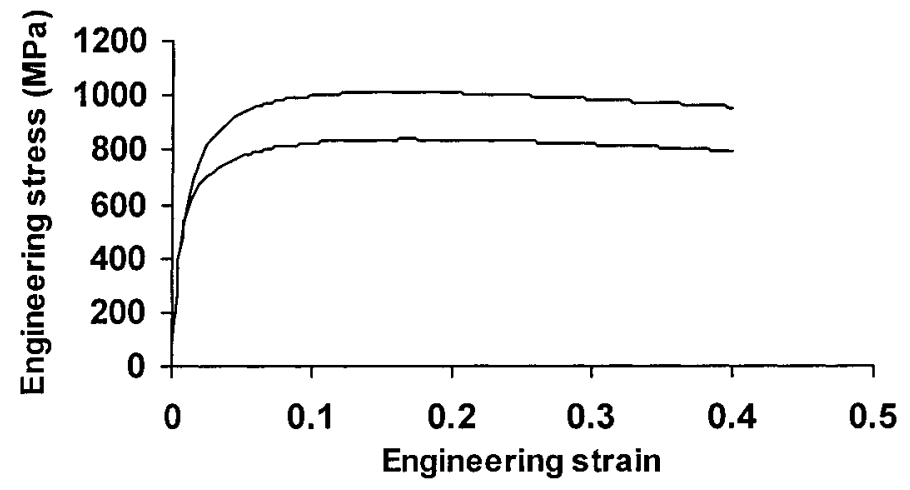

(c)

Fig. 7.2: Predicted effect of carbon dilution of martensite phase on the behavior of DP-steels for $V_{\mathrm{m}}$ (a) $31.4 \%$, (b) $34.1 \%$ and (c) $55.4 \%$. 


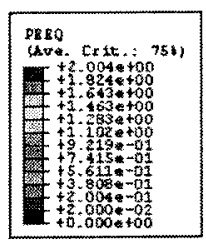

(a)
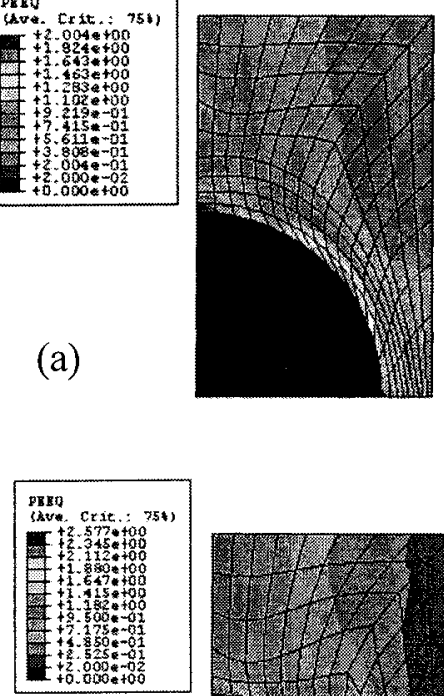

(b)
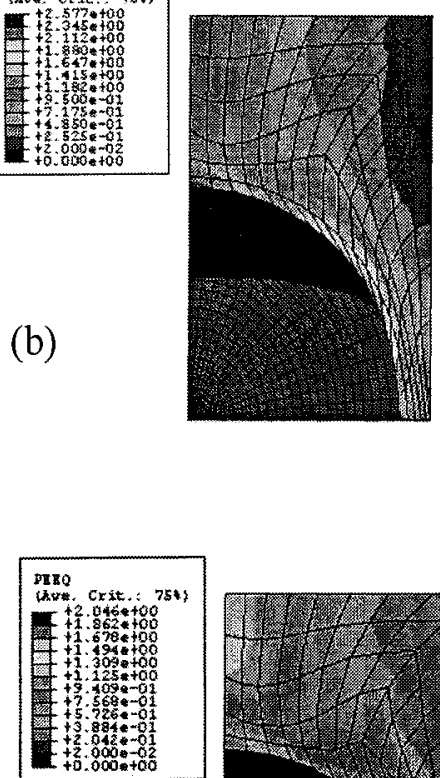

(c)
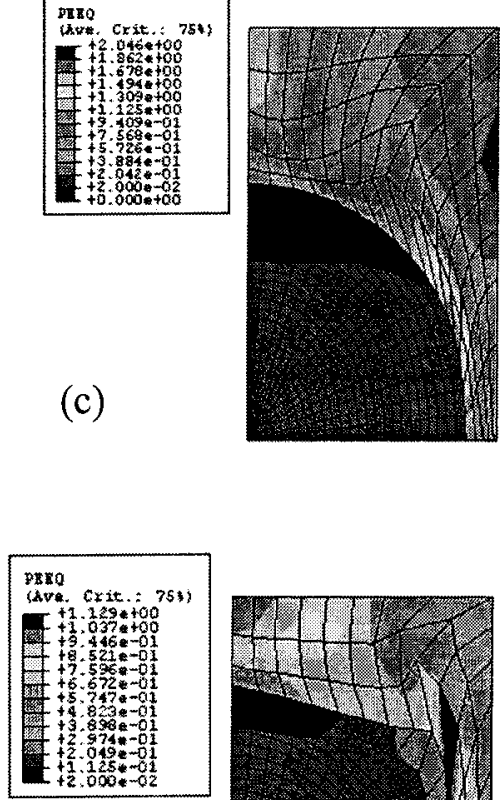

(d)

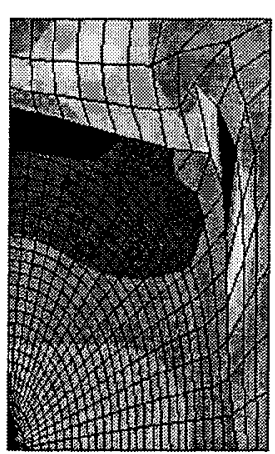

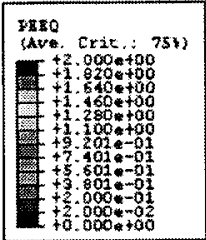
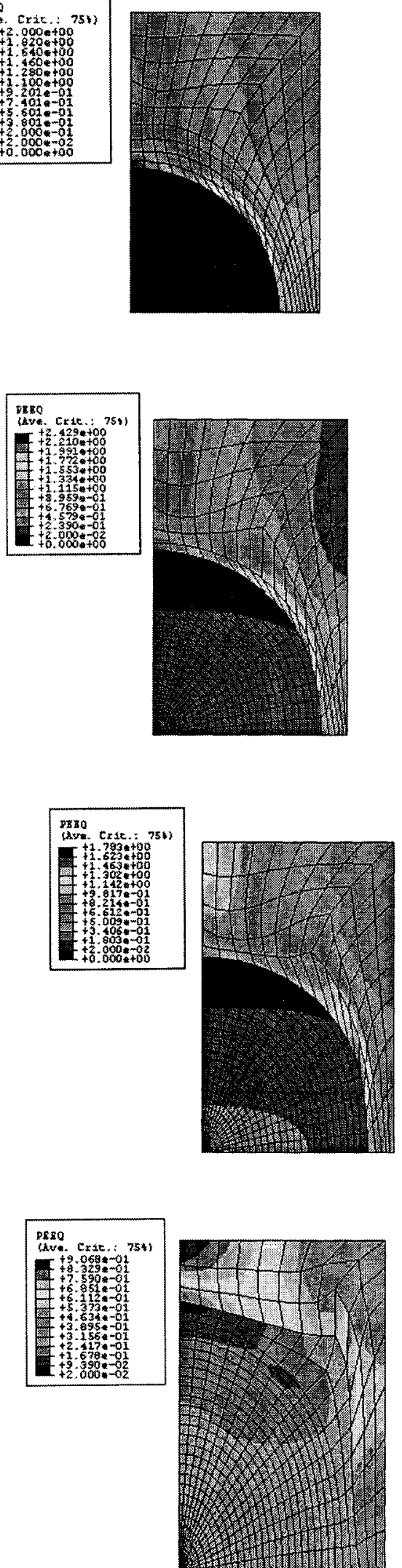

Fig. 7.3: Contours of plastic strain with (left) and without (right) carbon dilution effect for $V_{\mathrm{m}}$ (a) $20.1 \%$, (b) $31.4 \%$, (c) $34.1 \%$ and (d) $55.4 \%$. 


\subsection{Comparison Between Experimental Results and Micro Mechanical Model Predictions:}

In this section the actual constitutive material behavior of the constituents are used to model the material being considered to quantitatively test the model's ability to capture the material behavior. Comparison between the experimental results and that predicted from the model will be conducted.

\subsubsection{Material Considerations:}

In the coming sections the material considerations are implemented in the axisymmetric model with $\mathrm{R}=1 / 2$ presented earlier in Chapter 6 and shown in Fig. 7.4. In the present comparison, the ferrite grain size in the DP-steels presented in Chapter 4 is approximately $13 \mu \mathrm{m}$ and that in the single ferrite phase is $17 \mu \mathrm{m}$, using the lineal intercept method. Using the results of Morrison (1968), the difference in the yield strength is negligible, but in cases where this effect becomes important, it can easily be incorporated as will be shown later in this Chapter. The carbon content for each $V_{m}$ of interest is shown in table 7.1 .

A number of numerical expressions have been proposed for estimating the tensile properties of each single-phase material in multiphase steels (e.g. Morrison (1966) and Pickering (1978)). However, these empirical expressions are limited as they are obtained from experiments over limited ranges of chemical compositions and manufacturing processes. Other workers (e.g. Ishikawa et al. (2000)) have approximated the behavior of ferrite by measuring the hardness of the ferrite phase. They used the simple hardnesstensile strength relation to determine the ultimate strength of ferrite. In addition, they used the rule of mixtures to predict the yield stress and approximated a strain-hardening index to be able to describe the plastic stress-strain relation for the range of interest. The 


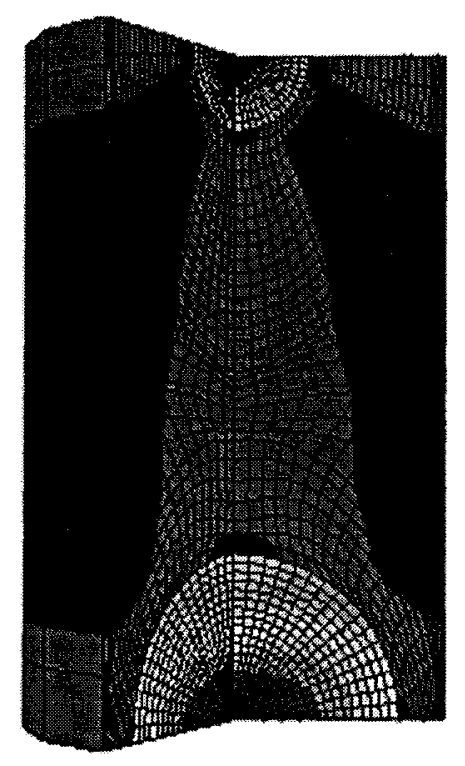

Fig. 7.4: Two martensite particles of ratio $R=1 / 2$ dispersed in a matrix of ferrite.

\begin{tabular}{|c|c|}
\hline$V_{\mathrm{m}}$ & $\mathrm{Wt} \% \mathrm{C}$ \\
\hline $14.0 \%$ & $0.50 \%$ \\
\hline $19.7 \%$ & $0.38 \%$ \\
\hline $34.0 \%$ & $0.23 \%$ \\
\hline
\end{tabular}

Table 7.1: Carbon content of martensite for each $V_{\mathrm{m}}$ tested experimentally.

above includes many estimations and approximations, which can't be used as standardized practice as they involve many personal judgment of the researcher. In addition, the hardness values using the Vickers hardness test are not reliable for twophase materials due to their large size compared to the microstructure, which give misleading measurements. In the development of the ferrite in this work, personal 
judgment was minimized as behavior is found using mechanical testing machines widely used and accepted.

The martensite material behavior is adopted again from Davies (1978) as martensite strength characteristics are carbon dependent only and thus it would be sufficient to know the carbon content to adjust martensite strength. The ferrite material behavior is obtained from the single ferrite phase made as shown in Chapter 4, which is used to describe the plastic material behavior in the micromechanical model. Alloying elements have considerable effect on the behavior of the material and this phase behavior has to be produced for any material when the prediction of the material behavior is sought.

The experimental behavior of the ferrite was fitted using two equations namely, a logarithmic equation and a power law equation as one equation is not able to characterize the material behavior throughout the range of interest and is mathematically expressed as follows:

$$
\sigma=\left\{\begin{array}{rccc}
920\left(\varepsilon_{0}+\varepsilon_{p}\right)^{0.2556} & & \text { for } & 0 \leq \varepsilon \leq 0.15 \\
109 \operatorname{Ln}\left(\varepsilon_{0}+\varepsilon_{\mathrm{p}}\right)+753.4 & \text { for } & & \varepsilon \geq 0.15
\end{array}\right.
$$

The fit between the experimental stress-strain curve and the equations above are depicted in Fig. 7.5. The figure shows three groups of curves, namely, the experimental curve, the fit curves, which are the power law equation referred to as (1) in equation 7.3 and the logarithmic equation referred to as (2) in equation 7.3. To facilitate better fitting, both the true stress-strain and the nominal stress-strain curves of the power law and the logarithmic equations were used as the nominal stress-strain curves can better describe the UTS and the uniform strain than the true stress strain curves. The single ferrite behavior has been chosen to be continuous from the instability point onwards. Large 
strains are considered because the ferrite phase experiences large plastic deformations on the micro structural level especially at the ferrite martensite interface.

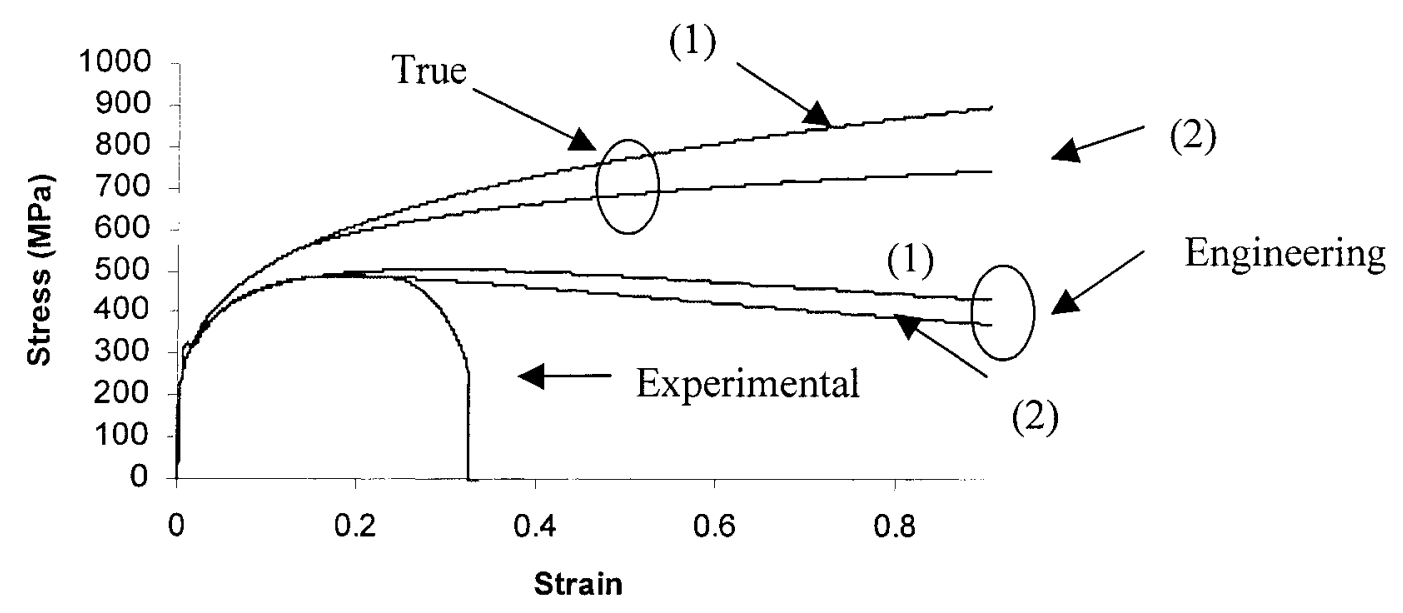

Fig. 7.5: Engineering and true stress-strain curves for single ferrite phase behavior fitted from the experimental results by two equations.

\subsubsection{Comparison of Significant Aspects of Material Behavior:}

The experimental results of three levels of $V_{m}$ are compared to predictions of the micro mechanical model for $\mathrm{R}=1 / 2$ as shown in table 7.2. True stress vs. true strain results are shown in Fig. 7.6 up to the maximum uniform elongation. Good agreement quantitatively in terms of strain hardening rate, ultimate strength, and uniform elongation is obtained for the three $V_{m}$, namely, $14 \%, 19.7 \%$ and $34 \%$, which indicates that the model developed has the intrinsic ability to capture the material behavior at different levels of $V_{m}$ of interest. 


\begin{tabular}{|c|c|c|c|c|}
\hline $\begin{array}{c}\text { Volume } \\
\text { fraction } \\
{[\text { Vm\%] }}\end{array}$ & $\begin{array}{c}\text { UTS [MPa] } \\
\text { calculated }\end{array}$ & $\begin{array}{c}\text { UTS [MPa] } \\
\text { experimental }\end{array}$ & $\begin{array}{c}\text { Uniform } \\
\text { elongation - } \\
\text { calculated }\end{array}$ & $\begin{array}{c}\text { Uniform } \\
\text { elongation - } \\
\text { experimental }\end{array}$ \\
\hline $14 \pm 0.56$ & 576 & $572-583$ & 0.17 & $0.152-0.155$ \\
\hline $19.7 \pm 0.86$ & 647 & $636-640$ & 0.21 & $0.147-0.154$ \\
\hline $34 \pm 1.95$ & 770 & $783-801$ & 0.15 & $0.110-0.151$ \\
\hline
\end{tabular}

Table 7.2: Predicted and experimental values of the ultimate strength and the uniform elongation for the range of $V_{m}$ considered in this work.

To better illustrate the details of the strain hardening, ultimate strength and uniform elongation, the curves were redrawn as engineering stress-strain curves as shown in Fig. 7.7. It can clearly be seen that the predicted values and the experimental values are very close.

An important characteristic of DP-steels is their high UTS to yield ratio or high strain hardening rate, which is particularly attractive for many forming processes. Fig. 7.8 depicts the strain-hardening rate vs. engineering strain at the three $V_{m}$ considered in this work. Due to vibration of signals in the MTS machine, the experimental results show a band of points for every 0.5 seconds. The model predictions at all levels of $V_{m}$ agree well with the experimentally observed results. The contours of the equivalent plastic strain for the three levels of $V_{\mathrm{m}}$ are shown in Fig. 7.9-1 and Fig. 7.9-2 for the set of $V_{\mathrm{m}}$ presented above. 


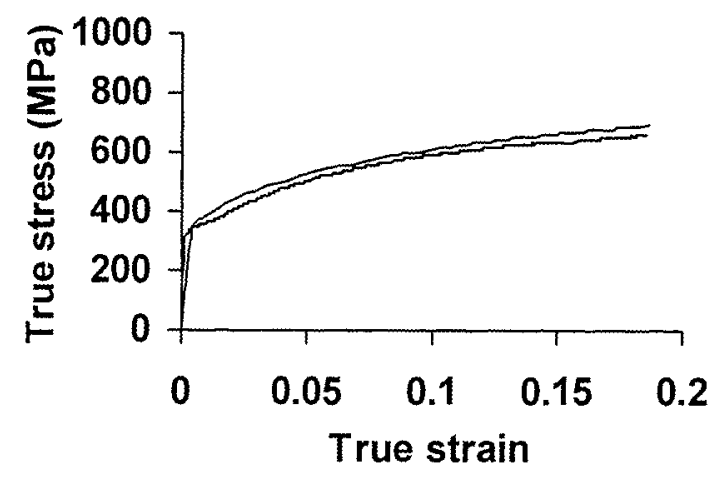

(a)

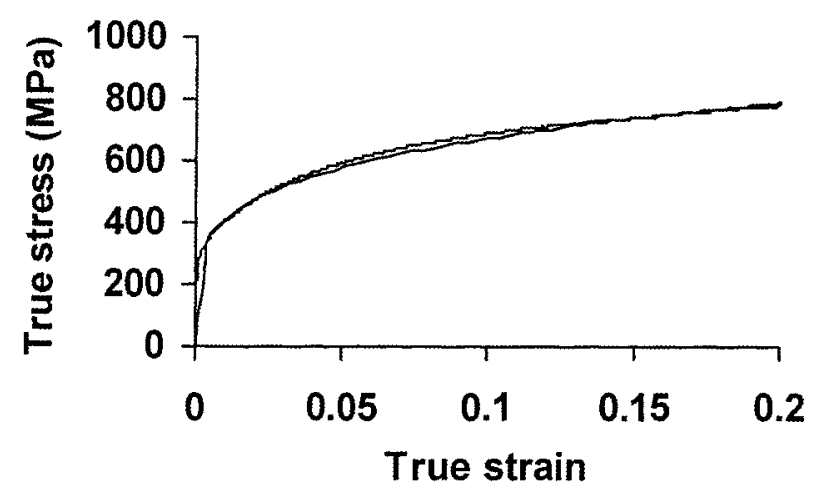

(b)

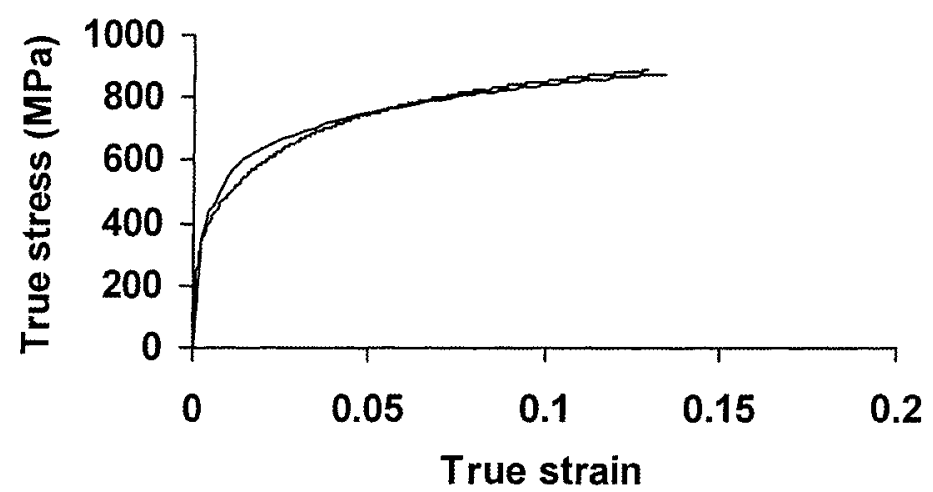

(c)

Fig. 7.6: True stress-strain diagrams for $V_{m}$ (a) $14 \%$, (b) $19.7 \%$ and (c) $34 \%$. 


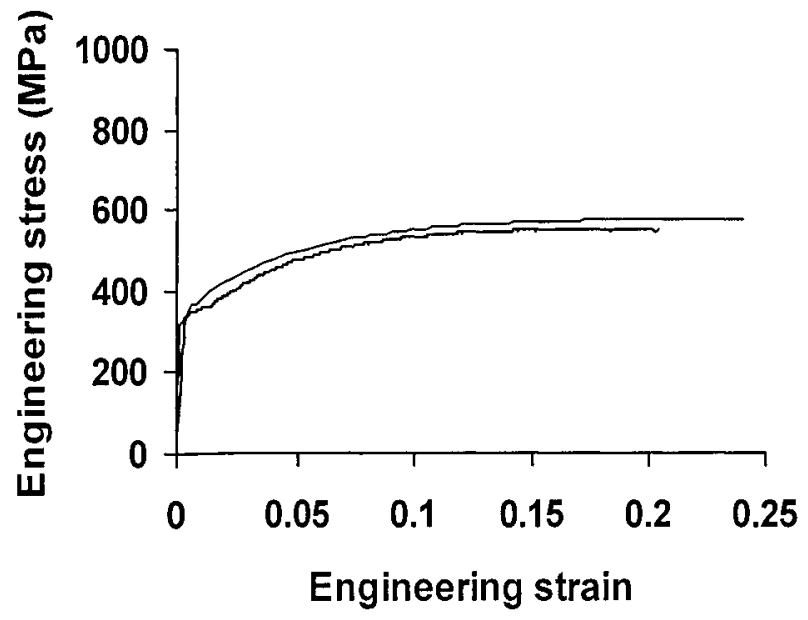

(a)

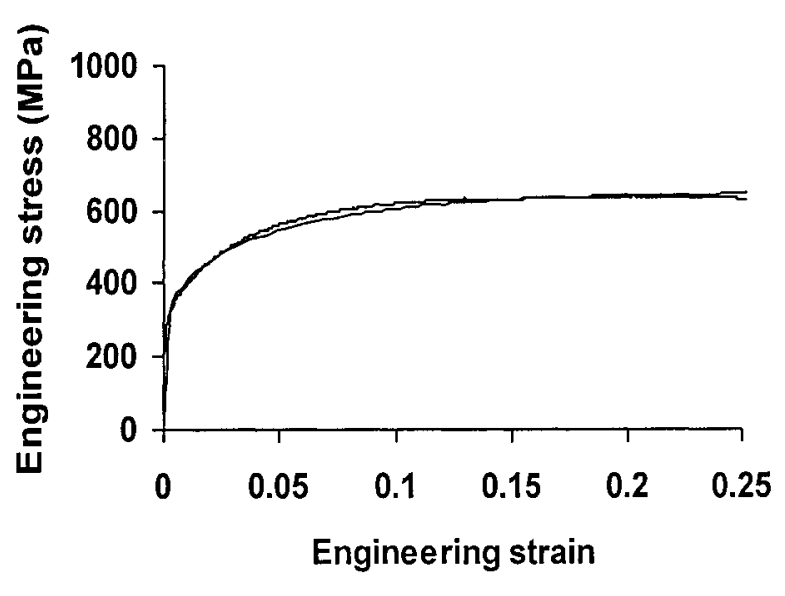

(b)

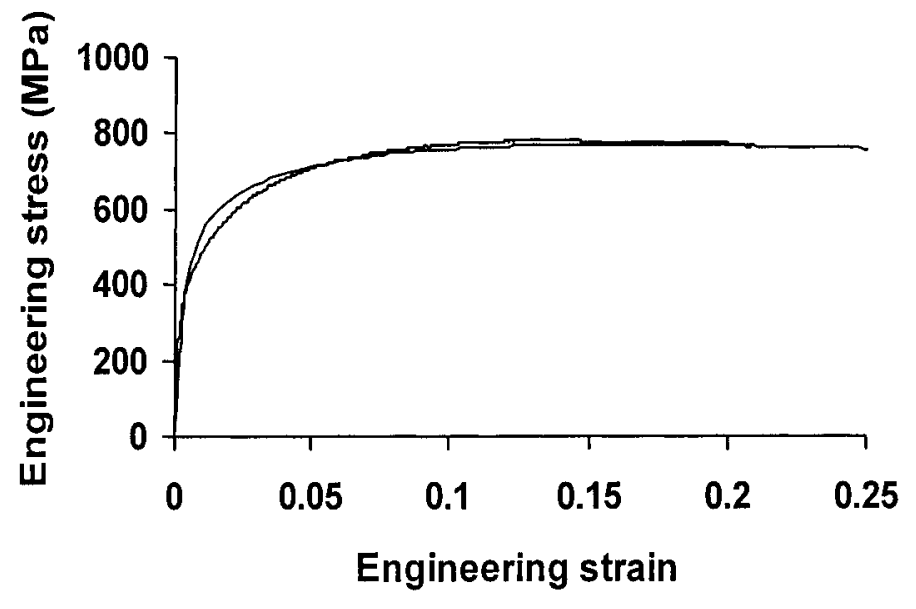

(c)

Fig. 7.7: Engineering stress strain diagrams for $V_{m}$ (a) $14 \%$, (b) $19.7 \%$ and (c) $34 \%$. 


\subsubsection{Discussion and Conclusion of the Comparison:}

The two particle axisymmetric model with $\mathrm{R}=1 / 2$ coupled with carbon dilution can well predict the experimental results as manifested in Fig. 7.6 and/or Fig. 7.7. The model captures the essential mechanical properties such as the UTS and the uniform elongation as shown in table 7.2. In addition, the deformation of the material shows several noticeable mechanisms:

1) At low $V_{\mathrm{m}}$, the model shows insignificant plastic deformation of the martensite particles (see Fig. 7.9-1) in agreement with Shen et al. (1986) where they reported that martensite particles did not experience any plastic deformation at low $V_{\mathrm{m}}$. This can clearly be seen from Fig. 7.9-1-a where the model displays negligible plastic strain at nominal strains of $10 \%, 20 \%$ and $30 \%$.

2) At intermediate and high $V_{\mathrm{m}}$ as can be seen in Fig. 7.9-1 b and 7.9-2, the plastic deformation extends into the martensite particles, a phenomenon believed to be causing the steep stress-strain behavior of the material generating high strain hardening at these levels of $V_{\mathrm{m}}$ which enable the material to display high strength and increased resistance to necking or increased uniform elongation at the optimum intermediate $V_{\mathrm{m}}$. This, as well, is in agreement with observations by Shen et al. (1986) where they have noticed using the microscope equipped with a straining stage that at high $V_{\mathrm{m}}$ the martensite particles deformed plastically by deformation being extended through the interface between the ferrite and martensite particles after the ferrite phase experiences excessive plastic deformation. In agreement with the above observations, Fig. 7.9-1 b and Fig. 7.9-2 both show that the ferrite phase undergoes high plastic deformation at the interface between the ferrite and the martensite. It can also be seen at the different stages of nominal strains shown in Fig. 7.9-1 b and Fig. 7.9-2, that the martensite phase gets plastically deformed only after the ferrite gets excessively deformed and that the deformation extends into the martensite phase after almost all the ferrite phase gets deformed plastically in agreement with experimental observations made by Shen et al. (1986). 
3) At intermediate and high $V_{\mathrm{m}}$, as can be seen in Fig. 7.9-1 b and Fig. 7.9-2, the model shows two different deformation mechanisms occurring in the material. The larger particle is observed to experience plastic deformation and the smaller one undergoes no measurable plastic deformation. In other words, two distinct deformation mechanisms take place, namely, a particle strengthening mechanism at the smaller martensite particle and elastic-plastic deformation at the larger particle. This agrees well with the observation by Shen et al. (1986) as they have noticed that the weaker martensite particles only deformed plastically after necking has taken place.

4) Considerable heterogeneity in the distribution of plastic strain, not only between phases, but also within each phase as can be seen in the model predictions in agreement with what was reported by Shen et al. (1986) and Rashid and Cprek (1978).

5) The model captures the important phenomena of strain-hardening rate observed experimentally as shown in Fig. 7.8 a, b \& c for levels of $V_{\mathrm{m}}$ of $14 \%, 19.7 \%$ and $34 \%$ considered in this work, which is a very attractive phenomenon especially for forming processes. 

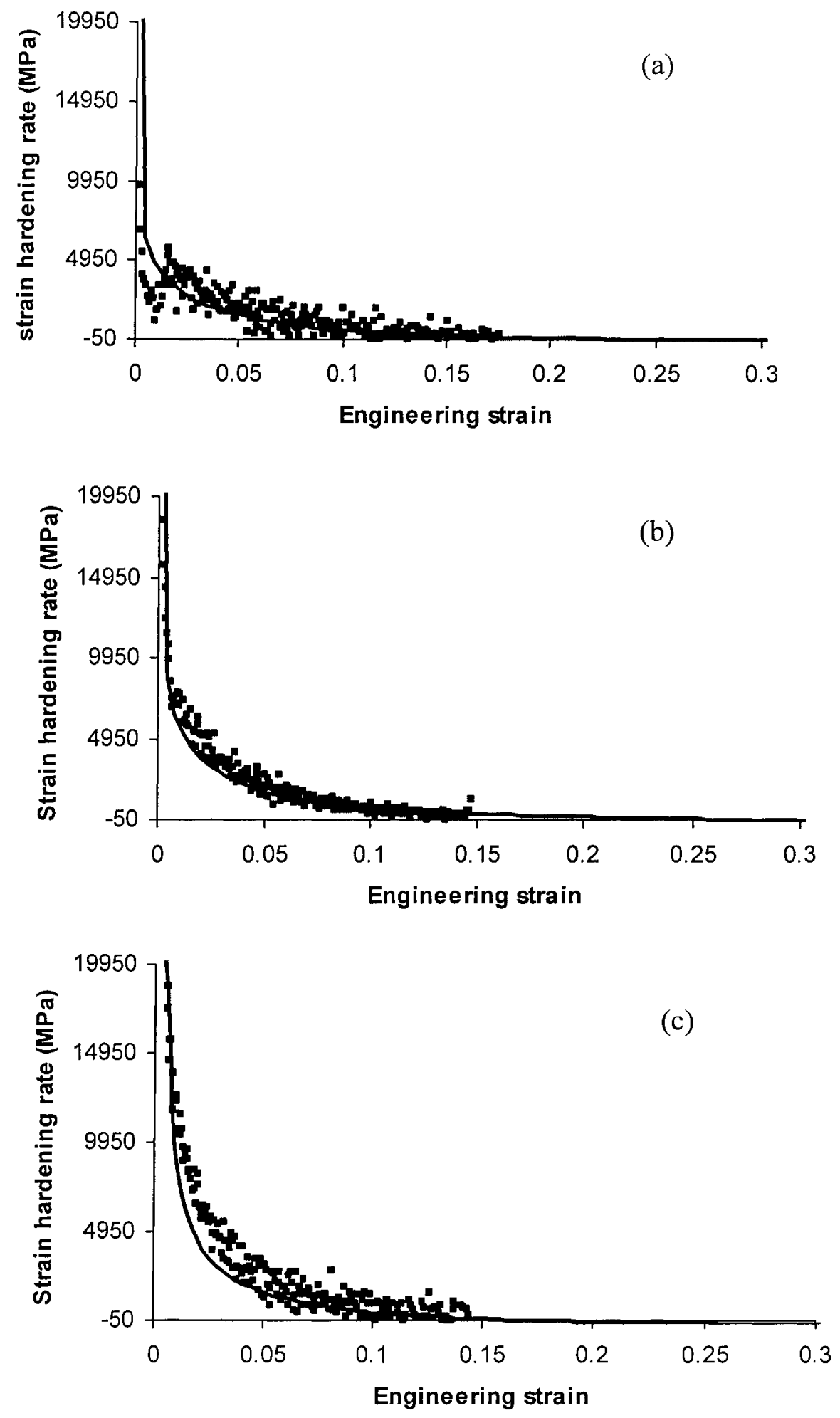

Fig. 7.8: Strain hardening rate for a) $V_{\mathrm{m}}=14 \%$, b) $V_{\mathrm{m}}=19.7 \%$ and $V_{\mathrm{m}}=34 \%$. 

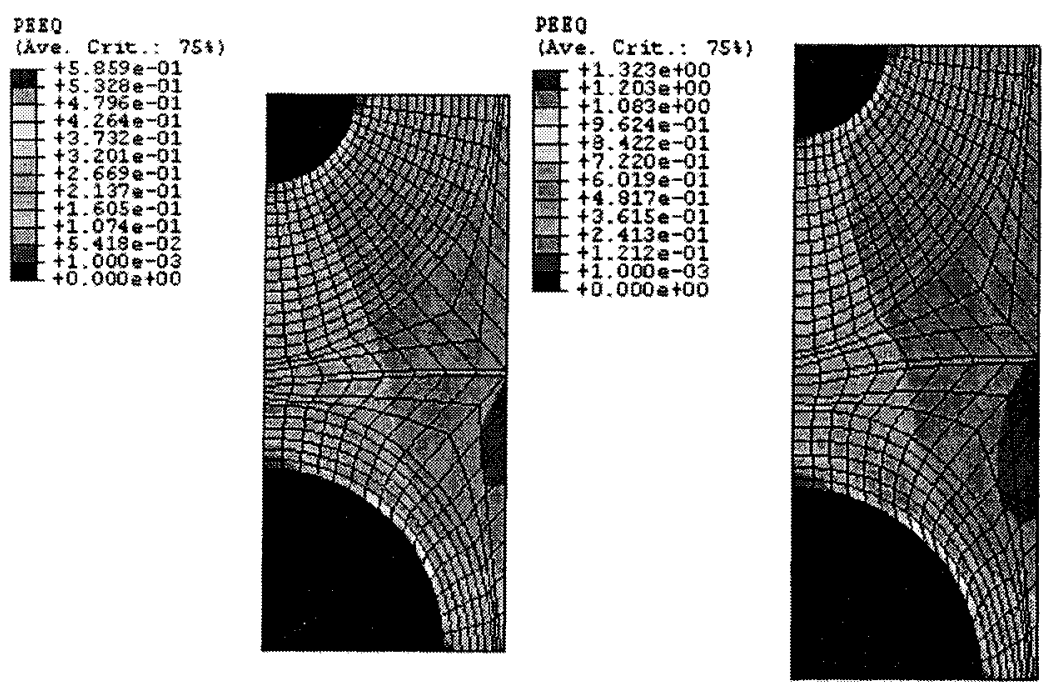

1580

3uve, Crit.: 75

$r+1.826=100$

$4+1.494 \mathrm{e}+00$

$1+1.162=10$

पt +9.965e +0

t. $+6.646=01$

$+1.669 \mathrm{e}-01$

$=+1.000$ e 03

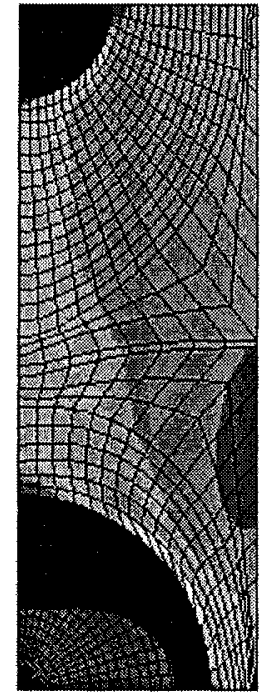

(a)

PREO (AVe, Crit: : 75*)
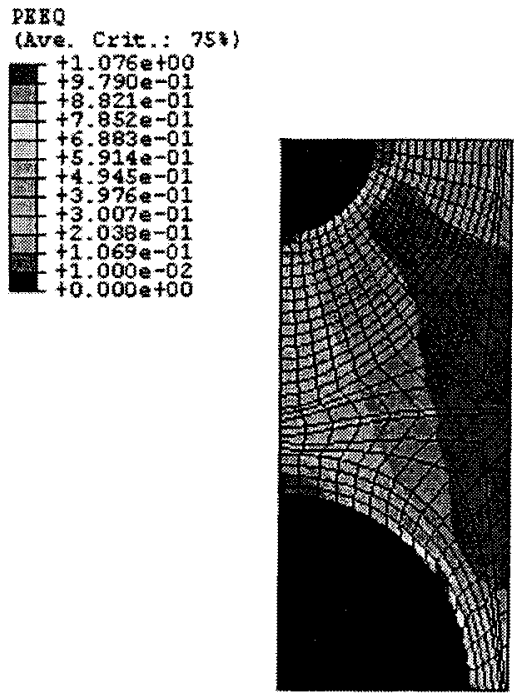

PBRO
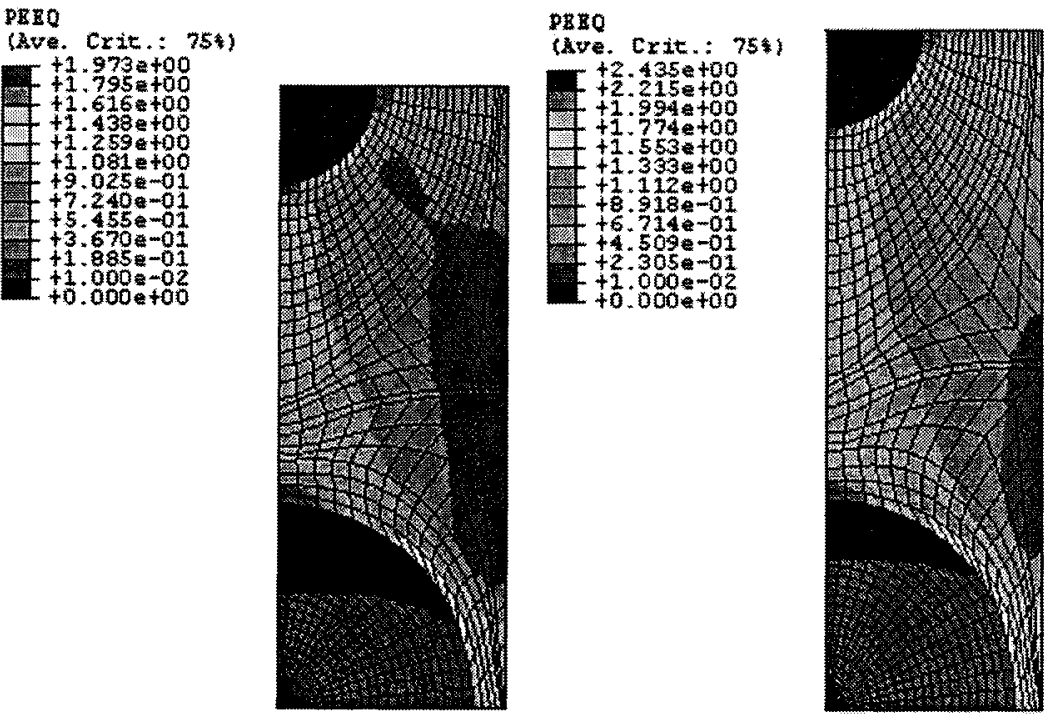

(b)

Fig. 7.9-1: Contours of the plastic strain for a) $V_{\mathrm{m}}=14 \%$, b) $V_{\mathrm{m}}=19.7 \%$ at nominal strains of $10 \%, 20 \%$ and $30 \%$. 

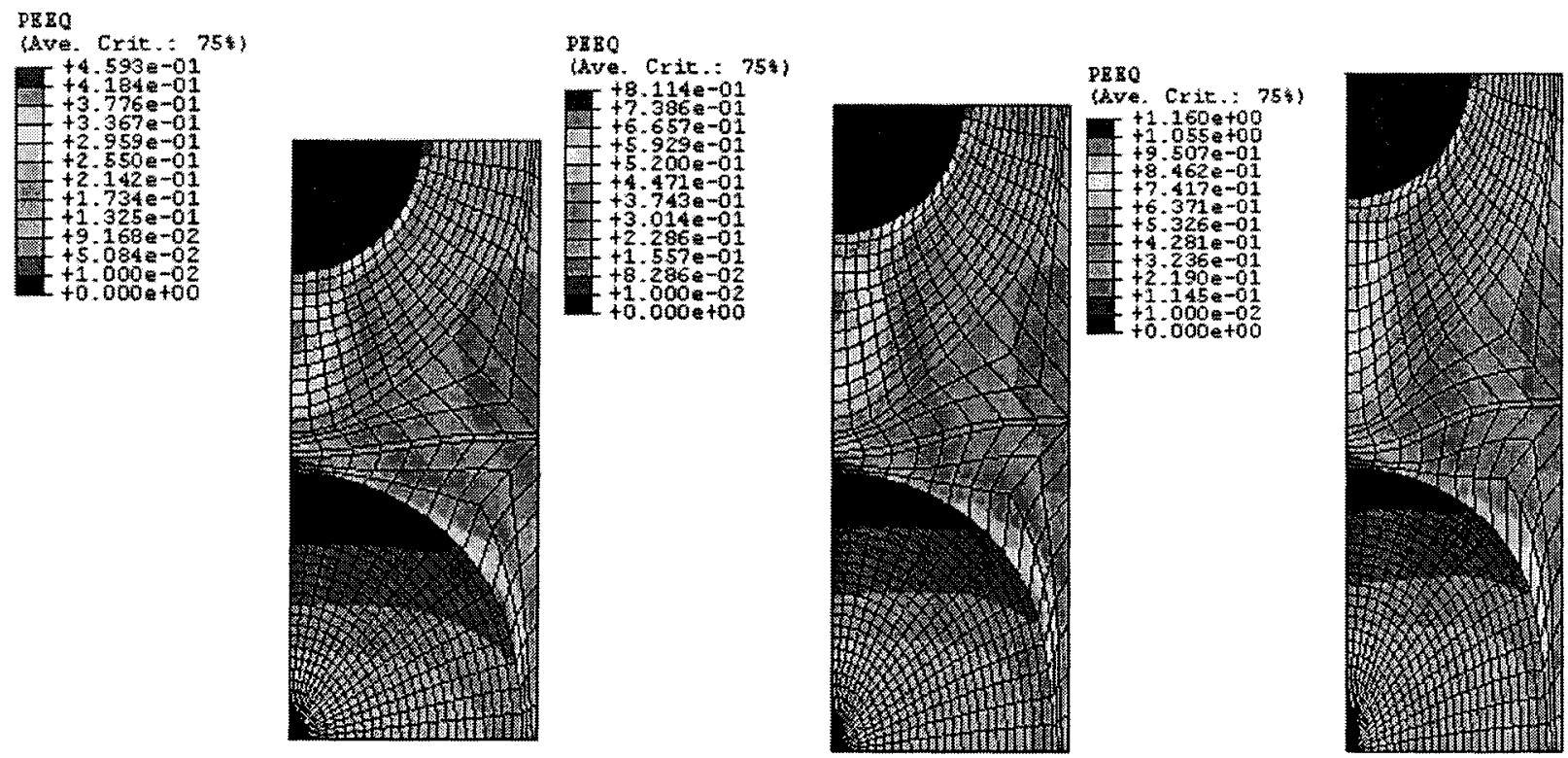

Fig. 7.9-2: Contours of the plastic strain for a) $V_{\mathrm{m}}=34 \%$ at nominal strains of $10 \%$, $20 \%$ and $30 \%$.

All the above predictions made by the $\mathrm{R}=1 / 2$ model agree very well with experimental observations made by Shen et al. (1986) and Rashid and Cprek (1978) and it has also been shown to predict the essential mechanical properties of DP-steels at different $V_{\mathrm{m}}$.

In the analysis above, the effect of the grain size on the mechanical properties was not accounted for as the average ferrite grain sizes of the microstructures at the three levels of $V_{\mathrm{m}}$ produced were close to that in the single ferrite material. The difference in the flow stress for the cases considered here compared to the single ferrite microstructure, which was determined from Morrison (1966), is negligible. To investigate how well the model can capture the grain size effect, one has to develop material microstructures with significantly different levels of ferrite grain sizes at the same $V_{\mathrm{m}}$, which cannot be accomplished through heat treatment alone and goes beyond the scope of this work. 
Results from the literature where different ferrite grain sizes are produced and tested are used to investigate and show the capability of the present model to capture the experimental observations, which is the subject of the next section. Although the alloying elements in the literature referenced is different from what is produced in this work, approximations are made to reproduce single ferrite material behavior for the material used in the literature, as will be discussed in detail shortly.

\subsection{Capturing the Effect of the Ferrite Grain Size on the Mechanical Properties of DP-Steels:}

The ferrite grain size effect as predicted by the model and observed experimentally (in the literature) is presented in this section to investigate the ability of the model to capture this essential material strengthening mechanism on the mechanical properties of the DPsteels. In order to predict the experimental results reported in the literature by the micromechanical model quantitatively, the constitutive behavior of the two constituents of the steel experimented are required. The martensite phase material behavior is carbon dependent only and thus it is fairly easy to approximate it's behavior for various $V_{\mathrm{m}}$ knowing carbon content of the steel considered as explained in section 7.1.1 taking into account the carbon dilution effect at the $V_{\mathrm{m}}$ considered. The single ferrite phase behavior depends on many factors including the alloying elements. It was difficult to get the same steel with the same alloying elements used in the literature and thus this phase behavior had to be approximated. The ferrite phase is estimated from properties tabulated for the steel considered by Chang and Preban (1985), where they have developed different ferrite grain sizes at the same $V_{\mathrm{m}}$ for various levels of $V_{\mathrm{m}}$.

The ferrite phase, as explained in section 2.1.1, can practically be called carbon free iron. The lowest carbon content steel used by Chang and Preban (1985) is $0.034 \%$ wt, which is considered low. This steel was used by Chang and Preban (1985) to produce the steel 
with $V_{\mathrm{m}}=3.3 \%$. In a backward calculation approach, the micromechanical model was used to approximate the single ferrite phase behavior. As a first approximation, the strain hardening index of the "assumed" ferrite was estimated from the yield strength and the UTS reported by Chang and Preban (1985) for the material at $V_{\mathrm{m}}=3.3 \%$ which is considerably small (close to ferrite behavior). Then, the ferrite material behavior was altered in a trial and error procedure in the micromechanical model until the model predicted the properties for the $V_{m}=3.3 \%$ tabulated by Chang and Preban (1985). The ferrite which enabled the micromechanical model to capture the behavior of the $V_{\mathrm{m}}=$ $3.3 \%$ which is at grain size of $59.1 \mu \mathrm{m}$ is used as the single ferrite phase subsequently.

The ferrite yield flow was corrected for each case of grain size considered based on the Hall-Petch equation shown below:

$$
\sigma_{y}=\sigma_{y 0}+K_{y} d^{-\frac{1}{2}}
$$

Where $\sigma_{y o}$ was taken as $88.3 \mathrm{MPa}$ and $K_{y}$ was taken as $573.4 \mathrm{MPa} \sqrt{\mu m}$ from Morrison (1966). The ferrite behavior, which gave closest prediction to one of the reported $V_{\mathrm{m}}$ can be represented in equation form as follows:

$$
\sigma=K\left(\varepsilon_{0}+\varepsilon_{p}\right)^{n}
$$

Where $n$ and $K$ were approximated to be 0.1 and $470 \mathrm{MPa}$ respectively. The comparison of the predicted results for the ferrite-martensite aggregate and the experimental results for the DP-steel reported by Chang and Preban (1985) for the yield strength and ultimate strength are shown in Figs. 7.10, 7.11. 


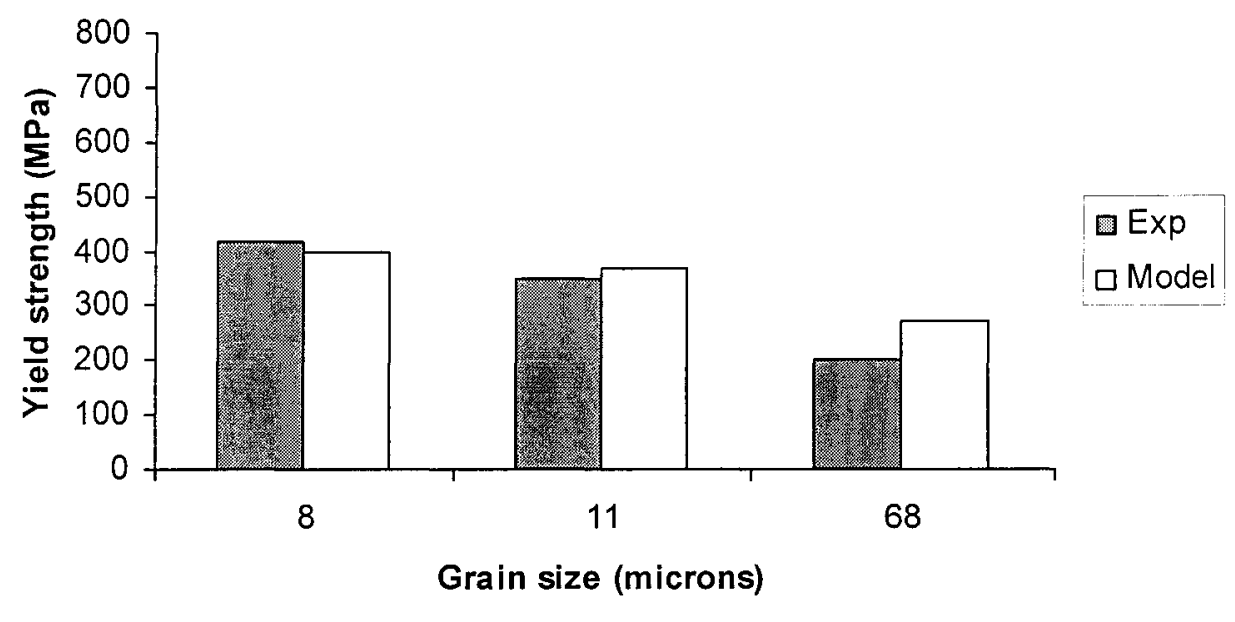

(a)

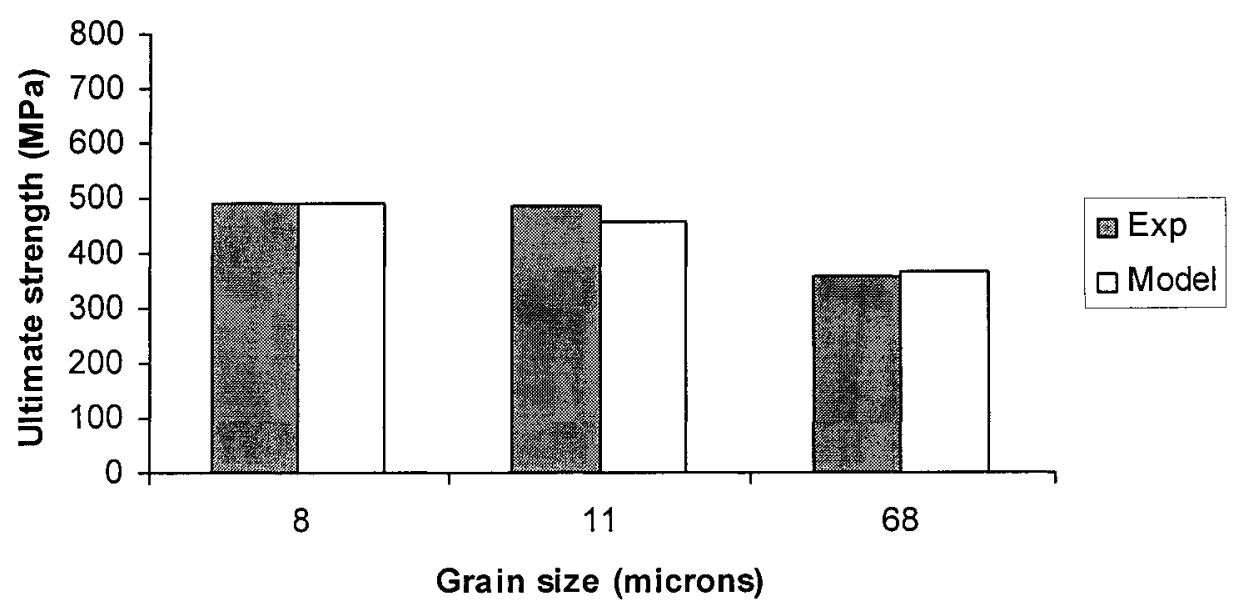

(b)

Fig. 7.10: Comparison between the model predictions and the experimental results from Chang and Preban (1985) of the (a) Yield strength (b) Ultimate strength for $V_{\mathrm{m}}=\mathbf{1 1 . 4 \%}$ at grain sizes depicted. 


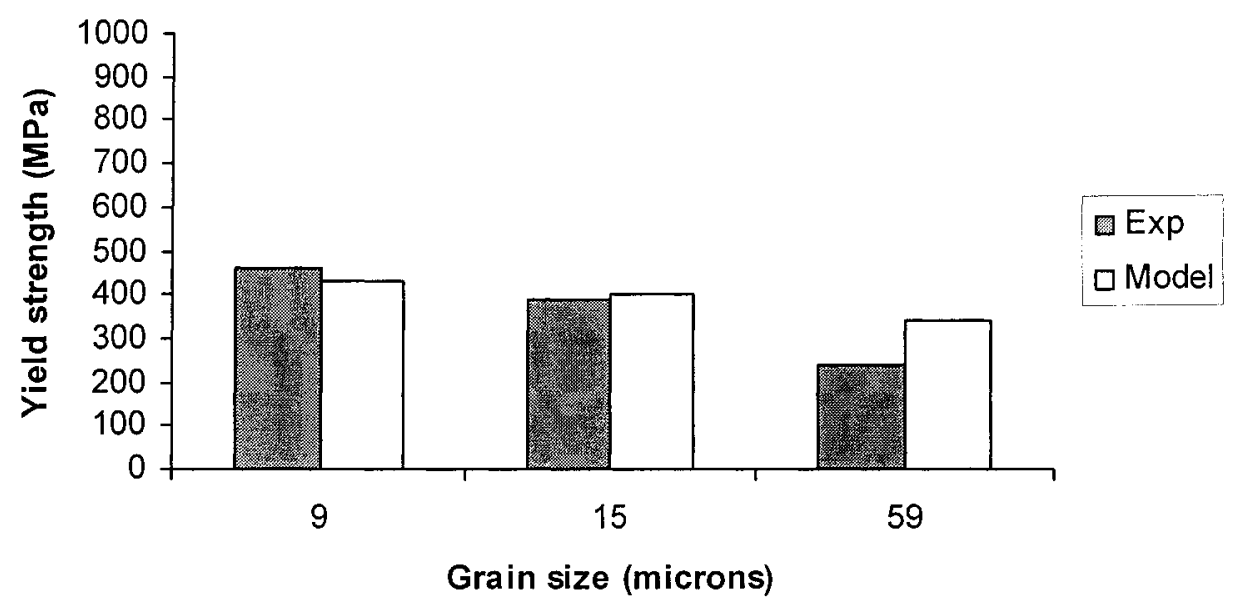

(a)

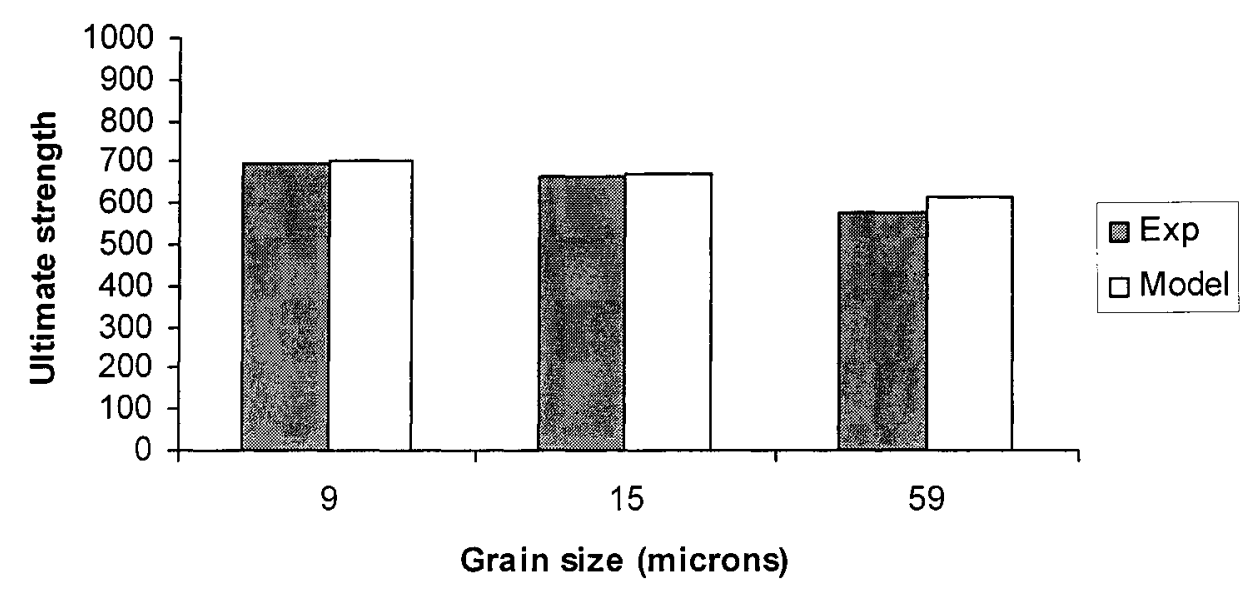

(b)

Fig. 7.11: Comparison between the model predictions and the experimental results from Chang and Preban (1985) of the (a) Yield strength (b) Ultimate strength for $V_{\mathrm{m}}=34 \%$ at grain sizes depicted. 
From Fig. $7.10 \mathrm{a} \& \mathrm{~b}$ for $V_{\mathrm{m}}=11.4 \%$ the results of the yield strength and the ultimate strength from the model compare very well with the experimental results for ferrite grain sizes of $8 \mu \mathrm{m}, 11 \mu \mathrm{m}$ and $68 \mu \mathrm{m}$. The same can be said for the results for $V_{\mathrm{m}}=34.4 \%$ at grain sizes of $9 \mu \mathrm{m}, 15 \mu \mathrm{m}$ and $59 \mu \mathrm{m}$ as depicted in Fig. $7.11 \mathrm{a} \& \mathrm{~b}$. The results above demonstrate that the micromechanical model developed in this work can capture the grain size effect satisfactorily.

\subsection{Constitutive Model Development:}

In addition to predicting properties, such as the UTS and uniform elongation, the micro mechanical model is used to predict the constitutive behavior of the aggregate material, which can then be used in numerical simulations. On the micro level, the model is not allowed to neck as it is assumed a continuous system of the microstructure, and thus it does not capture some macro level characteristics of deformation behavior such as necking. This effect has to be included separately and can be accounted for by using the material behavior output from the micro model as constitutive behavior for any realistic mechanical part to be tested in tension or compression. As an illustration, the true stress vs. true strain behavior predicted by the micro model, as shown in Fig. 7.6, is used to generate the constitutive model, which can be used in the numerical simulation of any geometry. The aggregate is considered as an elastic-plastic solid, where the flow stress, $\sigma$, is taken to be a function of the plastic strain, $\varepsilon_{p}$, according to the following:

$$
\sigma=K\left(\varepsilon_{0}+\varepsilon_{p}\right)^{n}
$$


The parameters $K, \varepsilon_{0}$, and $n$ are determined from a fit of the true stress vs. true strain response from the micro mechanical model from a strain of $0.2 \%$ to the uniform elongation. Resulting parameters are shown in table 7.3 for the three levels of $V_{m}$.

\begin{tabular}{|c|c|c|c|}
\hline $\mathrm{V}_{\mathrm{m}}$ & $\mathrm{K}$ & $\mathrm{n}$ & $\varepsilon_{0}$ \\
\hline 14.0 & 994 & .2118 & .0102 \\
\hline 19.7 & 1131 & .2245 & .0129 \\
\hline 34.0 & 1220 & .1688 & .0037 \\
\hline
\end{tabular}

Table 7.3. Parameters used to describe the macroscopic material behavior. 


\section{CHAPTER}

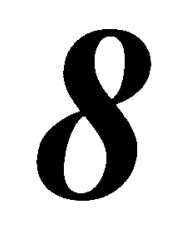

\section{MODELING THE DUCTILE FAILURE OF DP- STEELS BY VOID NUCLEATION, GROWTH AND COALESCENCE}

Thus far, modeling DP-steels has been performed in order to predict the deformation behavior of the material. As demonstrated in Chapter 7 this has been done quite successfully, which enabled the prediction of the yield strength, UTS, uniform strain and the strain hardening index which are the essential parameters needed to describe the deformation of any material. In the previous treatments there were no fracture criteria employed and therefore the model could not predict the limiting strain value (fracture) of the aggregate under tensile loading. 
The ductile failure of DP-steels occurs by the process of void nucleation, void growth and finally void coalescence as shown in general in Chapter 2.1.4 and specifically for the material at hand in Chapter 4.2.2. For a void containing material, the most widely known material damage model is the Gurson-Tvergaard model, which has been used comprehensively in the literature. This model was the first to account for material softening reflected in the yield function, which was a great success in modeling ductile material failure. On the other hand, due to some drawbacks, this model went through a series of improvements by Tvergaard and others, because it greatly overpredicts strain to failure in real materials. Even with the improvements mentioned above, the model has no intrinsic ability to predict coalescence i.e. transition from homogeneous to localized deformation mode between voids. However, it has been shown by many workers that this model can adequately capture the failure behavior of many materials that fail by the process of void nucleation, growth and coalescence using the correct calibrated parameters in each case. This model, although used by many, is also criticized because of its many correcting parameters. However, the rational that there is no better model than this still prevails. The Gurson-Tvergaard model is a damage model which when coupled with the results of the micro mechanical model presented in this work will make up a complete material model.

\subsection{The Gurson-Tvergaard Model:}

The Gurson-Tvergaard model assumes a void-containing continuum material, which accounts for the voids in the yield function written as:

$$
\Phi=\left(\frac{\sigma_{e}}{\sigma_{y}}\right)^{2}+2 f_{v} q_{1} \cosh \left(\frac{3 q_{2} \sigma_{h}}{2 \sigma_{y}}\right)-\left(1+q_{3} f_{v}^{2}\right)=0
$$

Where $f_{v}$ is the void volume fraction, $\sigma_{e}$ is the effective stress, $\sigma_{h}$ is the hydrostatic stress and $\sigma_{y}$ is the yield stress of the fully dense matrix material. Tvergaard suggested 
calibrating parameters $q_{1}=1.5$ and $q_{2}=1$ and $q_{3}=q_{1}^{2}$ (Tvergaard $(1981,1982)$ ) to better capture experimental results. Since then, the parameters in the above equation have traditionally been adjusted by fitting the experimental results with finite element predictions using different values of the calibrating parameters in a trial and error fashion (e.g.: employing the Gurson-Tvergaard model in a simulation of a tensile bar and comparing the results to the experimental findings). The best fit was considered to be the proper values for the material considered. These values have been used regardless of the material properties, which suggests that the selection of these values (without certain criteria) was done blindly. Faleskog et al. (1998) reported that the "q" values for several studies made in previous works showed that the parameters selected in this way were not consistent for materials of different strain hardening index and yield strength to stiffness ratio, which provides clear evidence for the need of a robust scheme to determine these calibrating parameters.

Faleskog et al. (1998) and Geo et al. (1998) presented a procedure for choosing the proper calibration parameters $\left(q_{1}\right.$ and $\left.q_{2}\right)$ for different strain hardening rates of metal materials using a three dimensional model for moderate and high strain hardening materials as the parameters suggested by Tvergaard $(1981,1982)$ and Tvergaard and Needleman (1984) were not adequately capturing the real material behavior at different strain hardening rates. They followed a two-step calibration procedure; in the first step they used a three dimensional model containing a discrete spherical void model to get the values of the parameters and the second was to use those in the Gurson-Tvergaard model simulating a unit cell material which accounts for void existence in a smeared way.

In the process of determining the calibrating parameters, $q_{3}$ has been related to $q_{1}$ by $q_{3}=q_{1}^{2}$. Although this seems to have been widely accepted, none have shown a rational reason for this selection. The calibration made by Faleskog et al. (1998) has assumed the same. For the range of parameter values presented by Faleskog et al. (1998) and shown in Chapter 2 (table 2.1) the evolution of the failure in a tensile bar has been investigated 
using the deformation properties of the DP-steel with $V_{m}=34 \%$ to appreciate the sensitivity of the calibrating parameters in Gurson-Tvergaard model.

There are three parameters in Gurson-Tvergaard model to be determined, $q_{1}, q_{2}$ and $q_{3}$. The third parameter, $q_{3}$, as displayed in Fig. 8.1, is an insensitive parameter, as it does not change the predicted behavior significantly. It causes the sharp fall, or decline after the material loses the load carrying capacity. Fig. 8.1 shows the predictions of the model with $q_{3}$ equal to $2,2.25,2.5,5$ and 10 keeping the other two parameters at constant values of $q_{1}=1.5$ and $q_{2}=1$. It can clearly be seen that predicted results are insensitive. As a result, it can be chosen equal to $q^{2}{ }_{1}$ in agreement with other researchers.

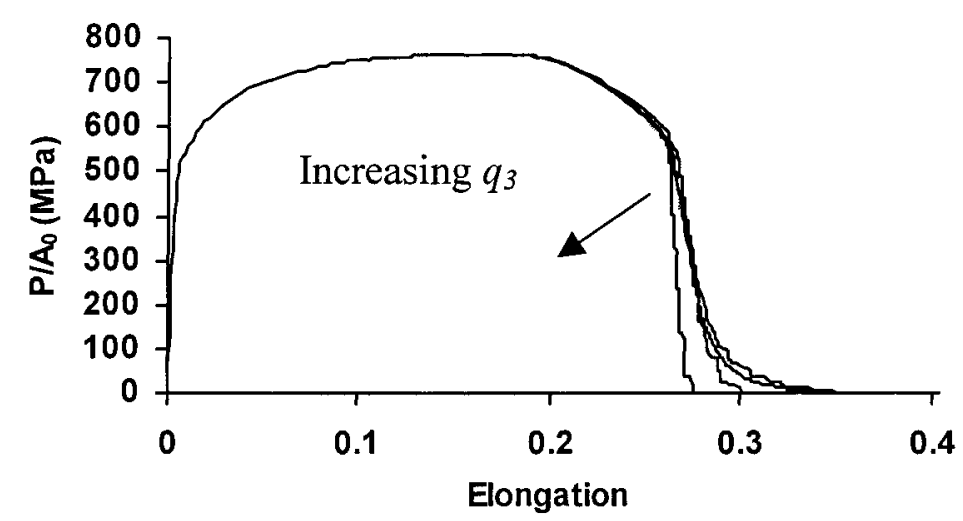

Fig. 8.1: Effect of the parameter ' $q_{3}$ ' keeping the other parameters constant.

The other two parameters have a significant effect on the predicted response of the material under consideration. This was investigated and shown in Fig. 8.2 and Fig. 8.3 below, which show that the parameters $q_{1}$ and $q_{2}$ have a significant influence on the response of the material at hand. The investigation was carried out using $q_{1}$ to be $1,1.1$, $1.2,1.3,1.4,1.7,1.8,2$ keeping $q_{2}=1$ and $q_{3}=2.25$ and using $q_{2}$ as $1,1.1,1.2,1.3,1.5$ keeping $q_{1}=1.5$ and $q_{3}=2.25$. 
The figures also show that the parameters $q_{1}$ and $q_{2}$ are sensitive as changing $q_{1}$ from 1 to 2 changes the strain at which the material loses it's load carrying capacity from a nominal strain of approximately $30 \%$ to a nominal strain of approximately $23 \%$. The same was true for $q_{2}$ but with slightly different values.

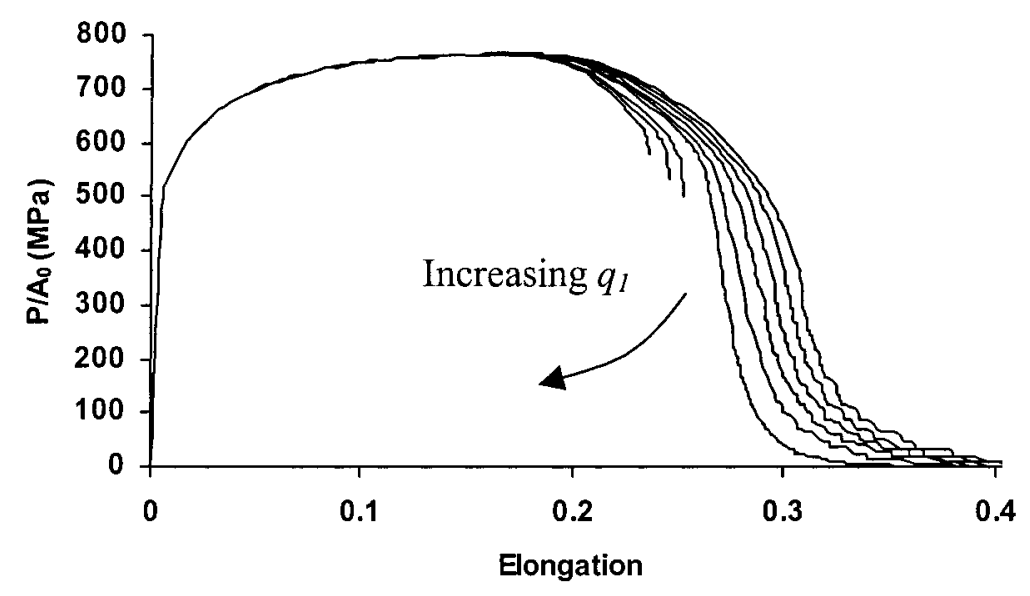

Fig. 8.2: Effect of ' $q_{1}$ ' keeping the other parameters constant.

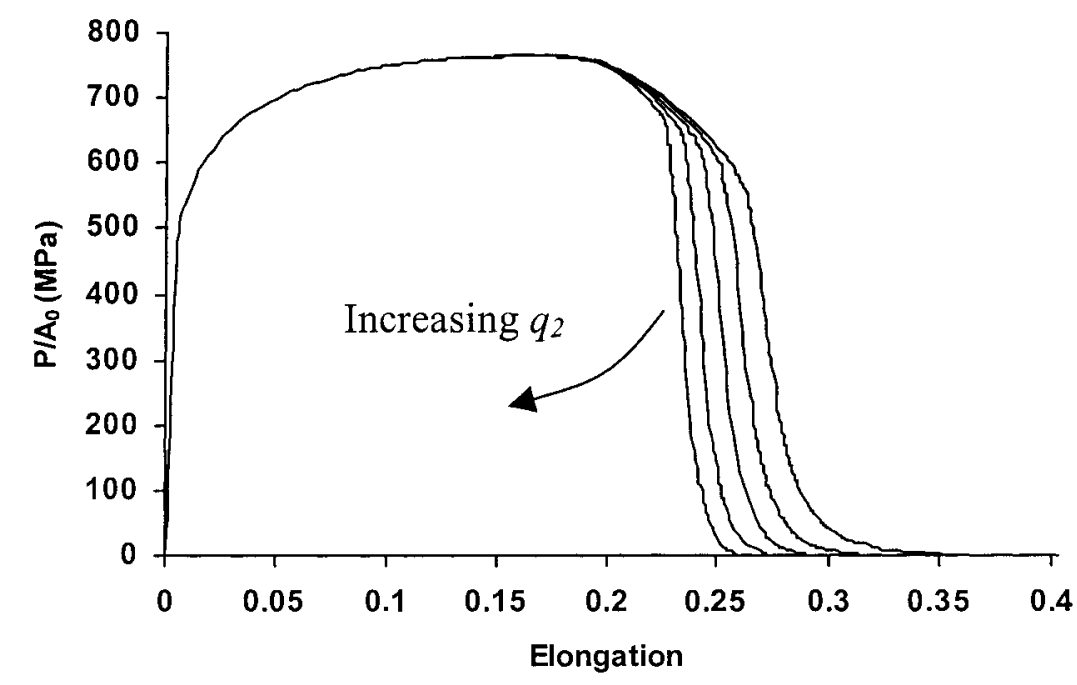

Fig. 8.3: Effect of ' $q_{2}$ ' keeping the other parameters constant. 
Therefore, the Gurson-Tvergaard model can be reduced to one with only two parameters which has the following form:

$$
\Phi=\left(\frac{\sigma_{e}}{\sigma_{y}}\right)^{2}+2 f_{v} q_{1} \cosh \left(\frac{3 q_{2} \sigma_{h}}{2 \sigma_{y}}\right)-\left(1+\left(q_{1} f_{v}\right)^{2}\right)=0
$$

The calibration procedure Faleskog et al. (1998) have proposed involves using a micro model with homogeneous matrix material containing a discrete void for different levels of strain hardening rates. Although the model they used captures localization occurring in plain carbon steels with voids, they do not necessarily represent MP-steels. The mechanics and mechanisms of deformation of DP-steels, as shown in this work, are different from plain carbon steels. Unlike the plain carbon steel, DP-steels undergo a series of mechanisms while deforming that introduces different strain localizations than the plain carbon steels, which should be considered when parameter calibration is considered. This demonstrates the need for calibrating the Gurson-Tvergaard model using the micro model developed in this work, which captures the mechanics and mechanisms of deformation taking place in DP-steels. This is the subject of the next section.

\subsection{Parameter Calibration Procedure:}

In the Gurson-Tvergaard model, the void volume fraction $\left(f_{v}\right)$ which is the current void volume fraction is the damage variable, and $\sigma_{y}$ is the current flow stress of the matrix material. There are two parameters in equation 8.2 that need to be determined in a procedure, which takes into account the void volume fraction as a damage criterion and loading condition, which should be consistent with what occurs in a tensile test bar during fracture. Therefore, these are the two essential failure criteria, which need to be observed in the process of obtaining the calibrating parameters in equation 8.2. This can be achieved by employing discrete voids in the micromechanical model, which well 
describes the deformation behavior of DP-steels to manifest void growth as the material is deformed. Since there are two parameters to be determined, two discrete void sizes can be employed in the micro mechanical model separately to get two different deformation responses. The stress triaxiality in the neck of a tensile bar reaches values in the range of 1.2 depending on the material properties. If the two discrete voids are employed in the micro mechanical model and the triaxiality is maintained in the triaxiality range corresponding to that in the neck of the tensile bar at failure, the parameters which are obtained by solving the yield function should represent the failure process of the material being considered.

A procedure to determine the calibrating parameters, which is developed in this work, is explained schematically in Fig. 8.4. The fully dense matrix material referred to in the Gurson-Tvergaard model will be the micromechanical model consisting of ferrite and martensite, at the specific $V_{m}$ for which the response is sought. The response of the micromechanical model is obtained for the two void sizes (void1 and void2). At a strain corresponding to triaxiality of 1.2 , the response of the micro model with void1 is used to get the variables $f_{v 1}, \sigma_{e l}, \sigma_{h 1}$ and $\sigma_{y 1}$. Following the same steps, the micromechanical model response with void2 is used to get $f_{v 2}, \sigma_{e 2}, \sigma_{h 2}$ and $\sigma_{y 2}$. When the above variables are inserted in equation 8.2 , it gives two equations with two unknowns $\left(q_{1}\right.$ and $q_{2}$ ), which can be solved.

\subsection{Cell Model Description:}

The proper stress triaxiality conditions in the micro model above is controlled by two springs which are fixed laterally and axially at the sides of the model as shown in Fig. 8.5. The boundary conditions are similar to what was previously used in the micro model except for the two springs, which are used to get proper triaxiality conditions. A specified displacement of node " $d$ " on the vertical spring $\left(\mathrm{k}_{2}\right)$ is applied and node "a" is fixed to develop stress triaxiality loading condition. The global true stress quantities used to describe the model predictions are: 


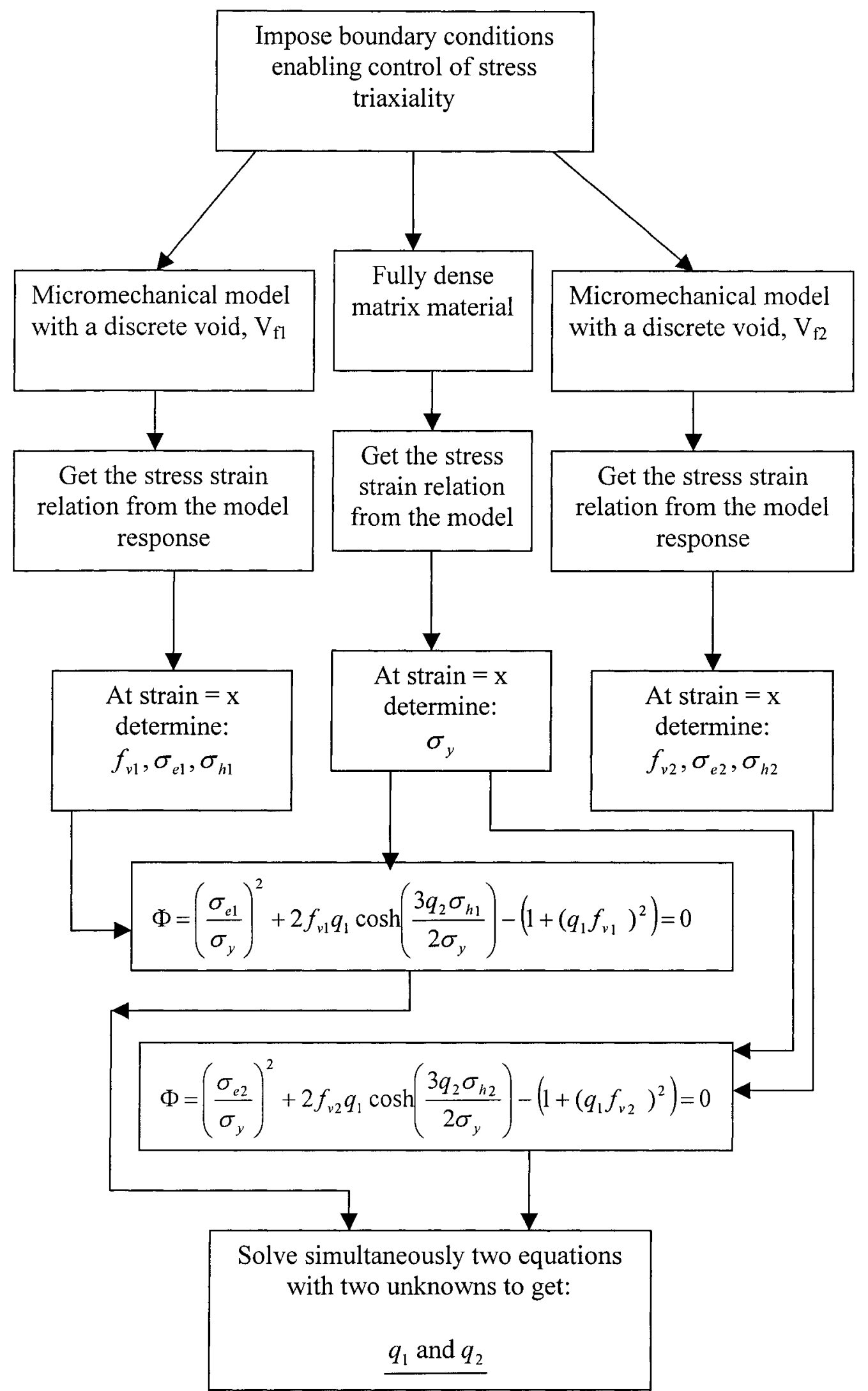

Fig. 8.4: Schematic diagram outlining the procedure to obtain the parameters from the Gurson-Tvergaard model. 


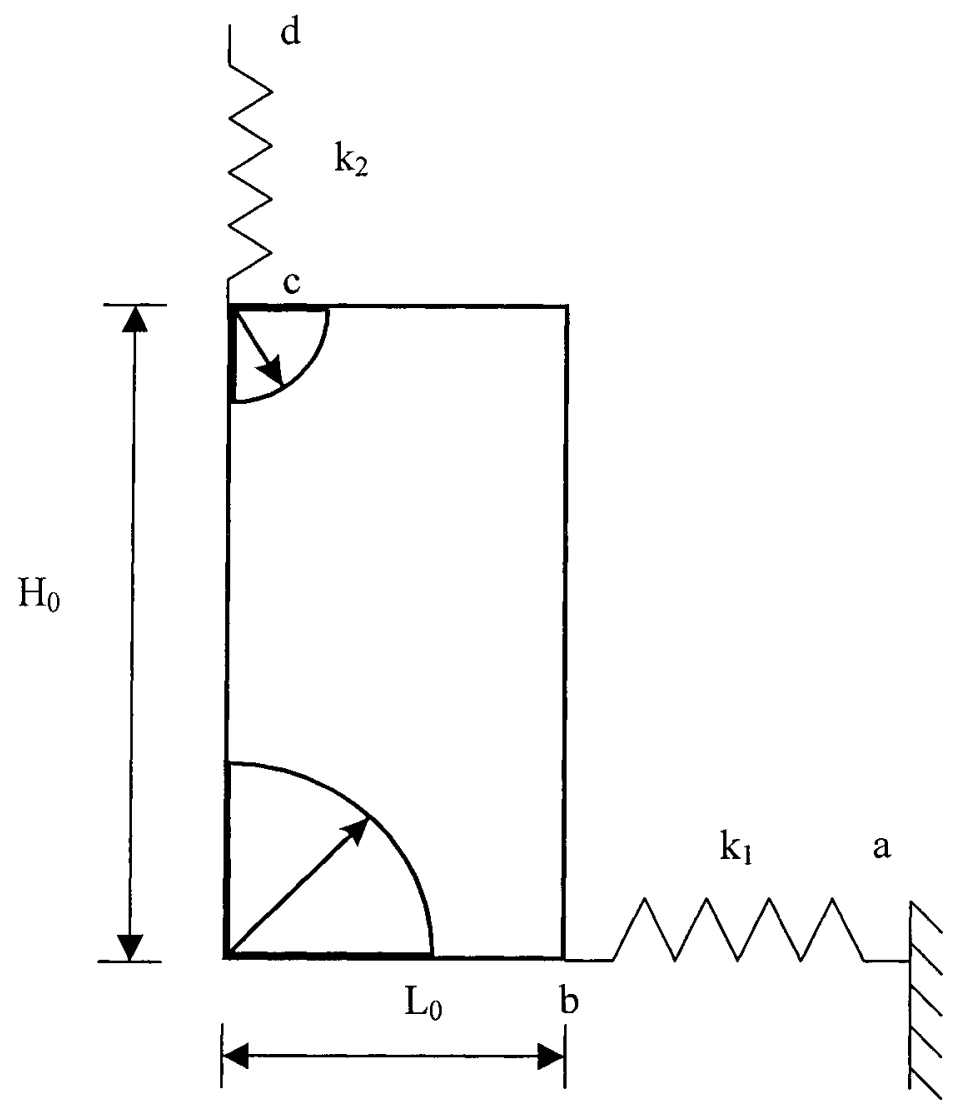

Fig. 8.5: Cell model with two springs and dimensions.

$$
\begin{aligned}
& S_{11}=\frac{F_{1}}{2 \pi\left(L_{0}+u^{b}{ }_{1}\right)\left(H_{0}+u^{c}{ }_{2}\right)} \\
& S_{22}=\frac{F_{2}}{\pi\left(L_{0}+u^{b}\right)^{2}}
\end{aligned}
$$

Where $F_{1}$ and $F_{2}$ are the concentrated loads in the radial and axial directions, respectively, $u^{b}{ }_{1}$ is the radial displacement of point " $\mathrm{b}$ " and $u^{c}{ }_{2}$ is the axial displacement of point " $c$ " on the model body. The other dimensions are shown in Fig. 8.5. The global effective and hydrostatic stresses can be expressed as follows: 


$$
\begin{aligned}
& S_{e}=\left|S_{22}-S_{11}\right| \\
& S_{h}=\frac{1}{3}\left(S_{22}+2 S_{11}\right)
\end{aligned}
$$

and the stress triaxiality, $\mathrm{T}$, can be defined as:

$$
T=\frac{S_{h}}{S_{e}}
$$

The macroscopic components of strain can be expressed as:

$$
\begin{aligned}
& E_{11}=\int_{L_{0}}^{L} \frac{d L}{L}=\ln \left(\frac{L}{L_{0}}\right)=\ln \left(\frac{L_{0}+u^{b}}{L_{0}}\right) \\
& E_{22}=\int_{H_{0}}^{H} \frac{d H}{H}=\ln \left(\frac{H}{H_{0}}\right)=\ln \left(\frac{H_{0}+u^{c}{ }_{2}}{H_{0}}\right)
\end{aligned}
$$

From which the effective strain can be expressed as follows:

$$
E_{e}=\frac{2}{3}\left|E_{22}-E_{11}\right|
$$

As shown in Chapter 2.1.4, a number of researchers assert that the failure process in DPsteels occurs by the decohesion of the interface between the ferrite matrix and the martensite particles. As a result, the discrete voids in the micro model are chosen to be at positions of maximum plastic strain, at the ferrite-martensite interface in order to describe the actual failure condition for the DP-steel. The voids are made in such a way that at the interface, extra nodes are inserted in order to disconnect the elements on the martensite 
side from the element on the ferrite side which imposes the required noncohesive condition simulating the condition of interface decohesion.

\subsection{Parameter Determination:}

The parameters are determined for three levels of $V_{m}$ considered in this work, namely, $V_{m}$ $=14 \%, 19.7 \%$ and $34 \%$. For each case two different martensite-ferrite surface interface decohesion sizes are applied in the micro model at similar stress triaxiality conditions by changing the spring stiffness, which changes the lateral to axial loading ratio. The stressstrain responses for the two void size cases for each $V_{m}$ considered are shown in Fig. 8.6. The fully dense matrix material response (model response with no void) for each $V_{m}$ is also added in this figure. The contours of equivalent plastic strain for effective strains corresponding to triaxiality, $\mathrm{T}=1.2$ are shown in Fig. 8.7 a, Fig. $8.7 \mathrm{~b}$ and Fig. $8.7 \mathrm{c}$ for $V_{m}$ $=14 \%, 19.7 \%$ and $34 \%$. The figures depict the void size and growth at the corresponding effective strains. The effective stress-strain curves are determined by evaluating the lateral and axial spring forces in each case to determine the axial and lateral stresses using equations 8.3 and 8.4 from which using equation 8.5 and 8.6 the effective and hydrostatic stresses are determined. The lateral and axial strains are determined using equations 8.8 and 8.9 from which using 8.10 the effective strain is calculated. In each void size case at a corresponding $V_{m}$, the stress triaxiality is measured using equation 8.7 , as the lateral to axial loading ratio is manipulated by changing the spring stiffness to obtain similar stress triaxiality for both cases up to effective strains of approximately $20 \%$. At a stress triaxiality of $\mathrm{T}=1.2$ for each void case the variables $f_{v}, \sigma_{e}, \sigma_{h}$ and $\sigma_{y}$ are determined. The void volume fractions were calculated using Catia 5.8. After all the variables were determined, the Gurson-Tvergaard equations for two cases of void volume fractions were solved using Maple 8 to obtain the parameters, $q_{1}$ and $q_{2}$. The variables obtained from 
the micro model using Fig. 8.6 and the void volume fraction using the contours in Fig. 8.7 are summarized in table 8.1. The parameters for each $V_{m}$ are summarized in table 8.2.

\begin{tabular}{|l|l|c|c|c|c|}
\hline & Void & $\boldsymbol{f}_{v} \%$ & $\sigma_{\boldsymbol{y}}$ & $\sigma_{h}$ & $\sigma_{e}$ \\
\hline \multirow{2}{*}{$V_{\boldsymbol{m}}=14 \%$} & & & & & \\
\hline & Void1 & $\mathbf{0 . 3 5 5 0 0}$ & $\mathbf{6 4 8 . 9 5 0}$ & $\mathbf{7 6 6 . 6 7 0}$ & $\mathbf{6 2 9 . 3 4 0}$ \\
\cline { 2 - 6 } $\boldsymbol{V}_{\boldsymbol{m}}=19.7 \%$ & Void2 & $\mathbf{1 . 0 8 4 1 3}$ & $\mathbf{6 4 8 . 9 5 0}$ & $\mathbf{7 2 1 . 8 0 0}$ & $\mathbf{5 9 4 . 5 0 0}$ \\
& Void1 & $\mathbf{0 . 4 9 5 0 0}$ & $\mathbf{7 4 1 . 0 0 0}$ & $\mathbf{8 5 3 . 9 9 0}$ & $\mathbf{7 1 4 . 1 7 0}$ \\
\cline { 2 - 6 } & Void2 & $\mathbf{1 . 7 3 6 6 5}$ & $\mathbf{7 3 4 . 0 0 0}$ & $\mathbf{7 8 8 . 4 2 2}$ & $\mathbf{6 5 1 . 1 5 0}$ \\
\hline \multirow{2}{*}{$\boldsymbol{V}_{\boldsymbol{m}}=\mathbf{3 4 \%}$} & Void1 & $\mathbf{0 . 5 2 1 5 7}$ & $\mathbf{6 4 9 . 3 9 0}$ & $\mathbf{9 8 3 . 1 6 3}$ & $\mathbf{6 2 9 . 4 2 1}$ \\
\cline { 2 - 6 } & Void2 & $\mathbf{0 . 8 3 3 4 3}$ & $\mathbf{6 6 7 . 4 6}$ & $\mathbf{9 5 3 . 3 8 0}$ & $\mathbf{6 1 0 . 3 5 0}$ \\
\hline
\end{tabular}

Table 8.1: Variables determined from stress-strain response of voided micro model.

\begin{tabular}{|c|c|c|c|}
\hline & $V_{\mathrm{m}}=14 \%$ & $V_{\mathrm{m}}=19.7 \%$ & $V_{\mathrm{m}}=34 \%$ \\
\hline$q_{1}$ & 1.900 & 2.331 & 2.574 \\
\hline$q_{2}$ & 1.210 & 1.012 & 0.905 \\
\hline
\end{tabular}

Table 8.2: Parameters determined by solving the Gurson-Tvergaard yield function. 

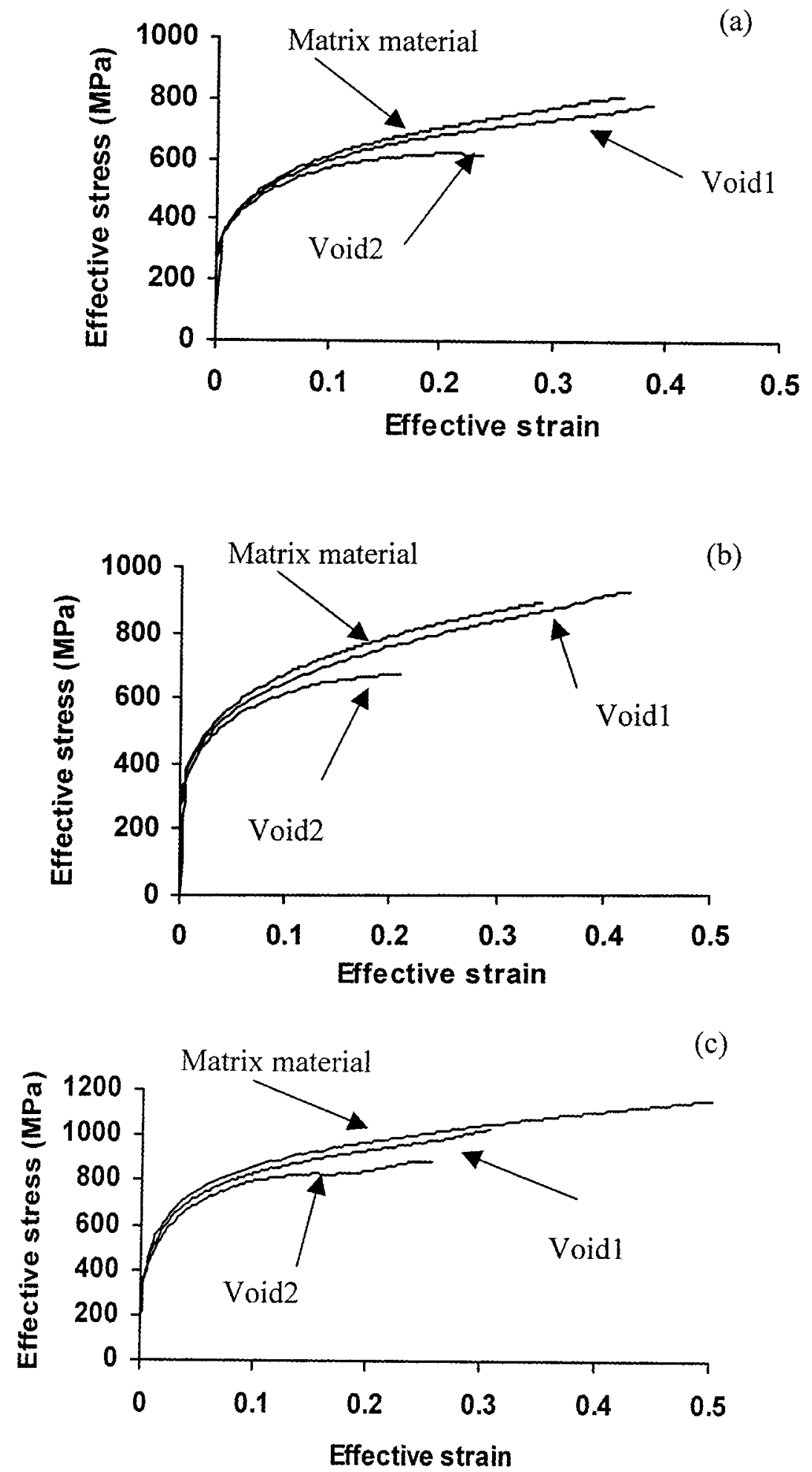

Fig. 8.6: Stress-strain response of the micro model for a) $V_{m}=14 \%$, b) $V_{m}=19.7 \%$ and c) $V_{m}=34 \%$. 


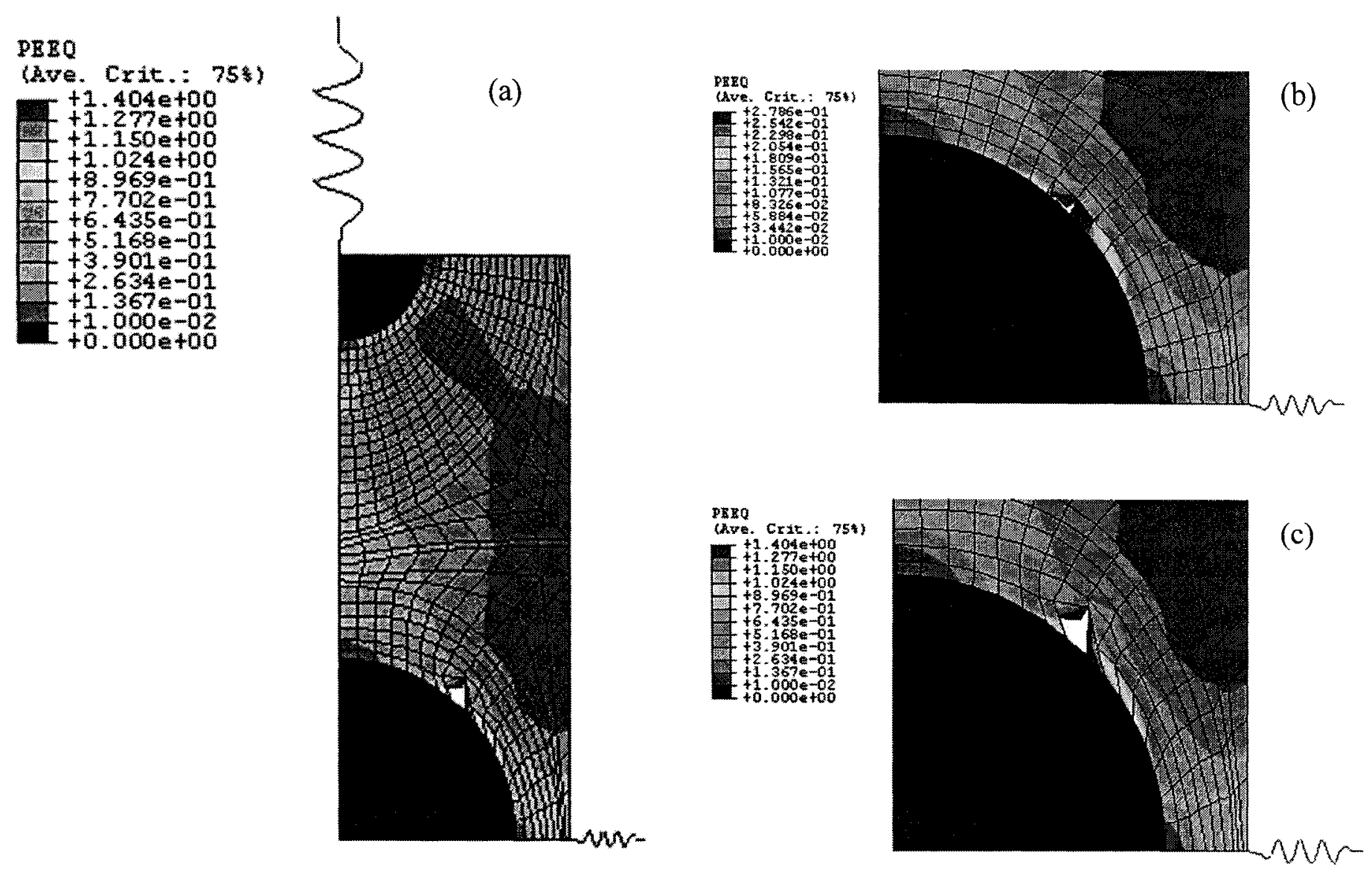

Fig. 8.7 a: a) Contours of plastic strain for $\mathrm{Vm}=14 \%$, void1 at $13.5 \%$ effective strain and details of void growth at b) $4 \%$ and c) $13.5 \%$ effective strain. 

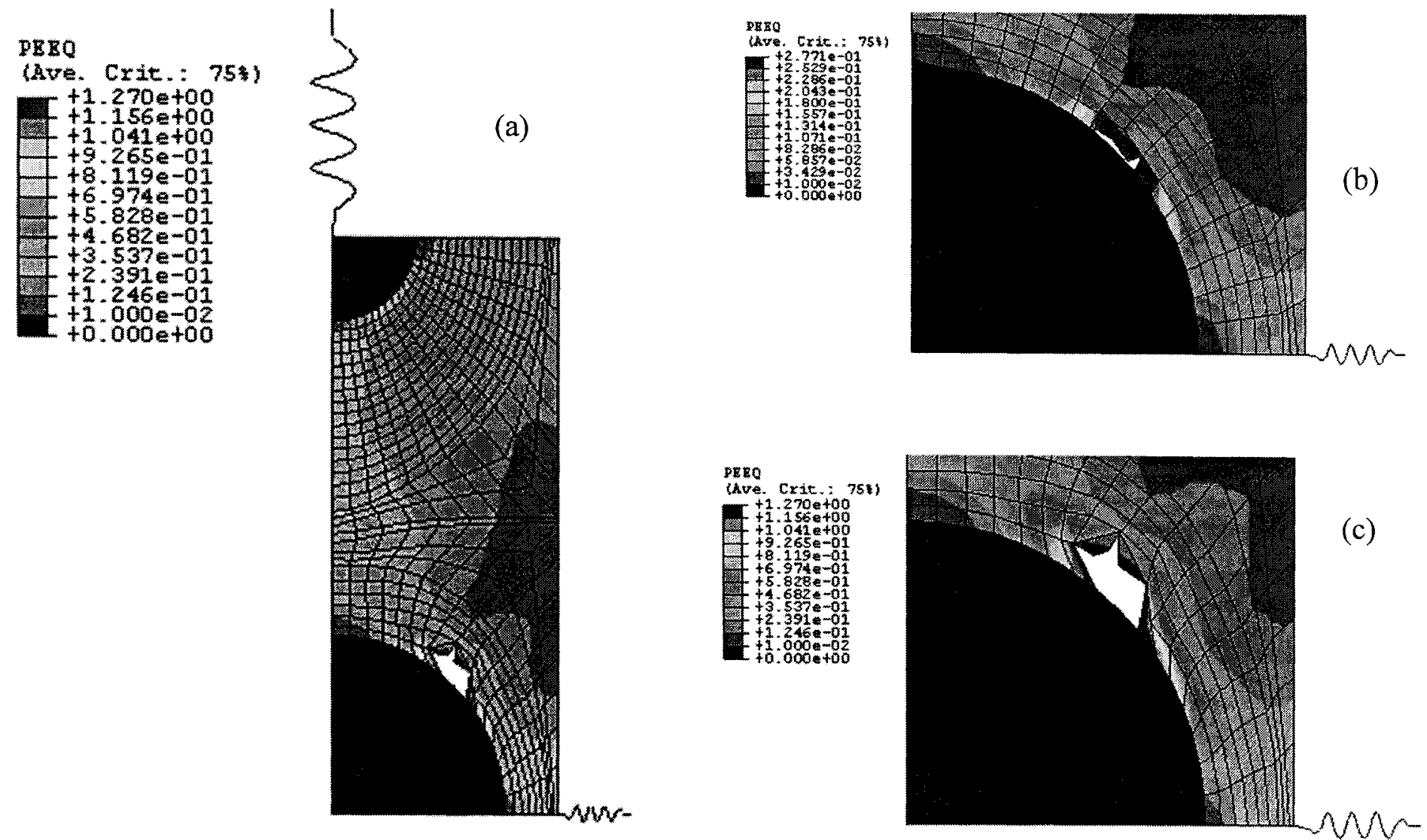

Fig. 8.7 b: a) Contours of plastic strain for $V_{m}=14 \%$ for void 2 at $13.5 \%$ effective strain and details of void growth at b) $4 \%$ and c) $\mathbf{1 3 . 5 \%}$ effective strain. 

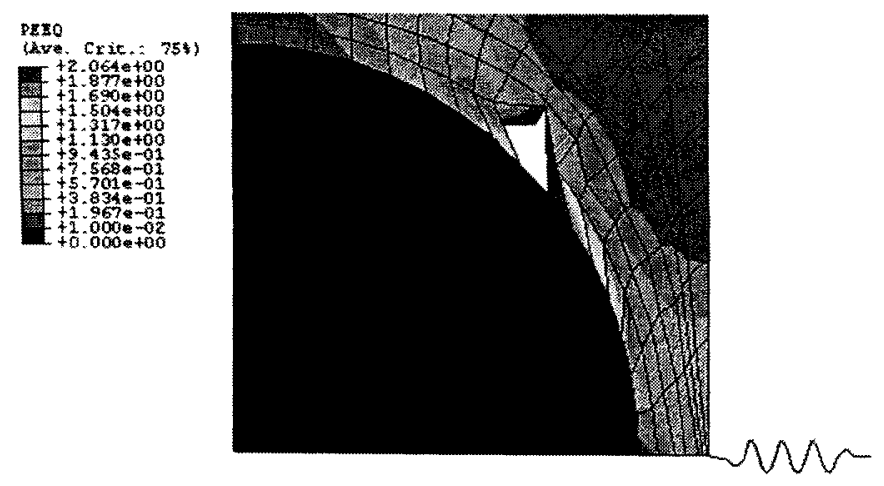

(a)
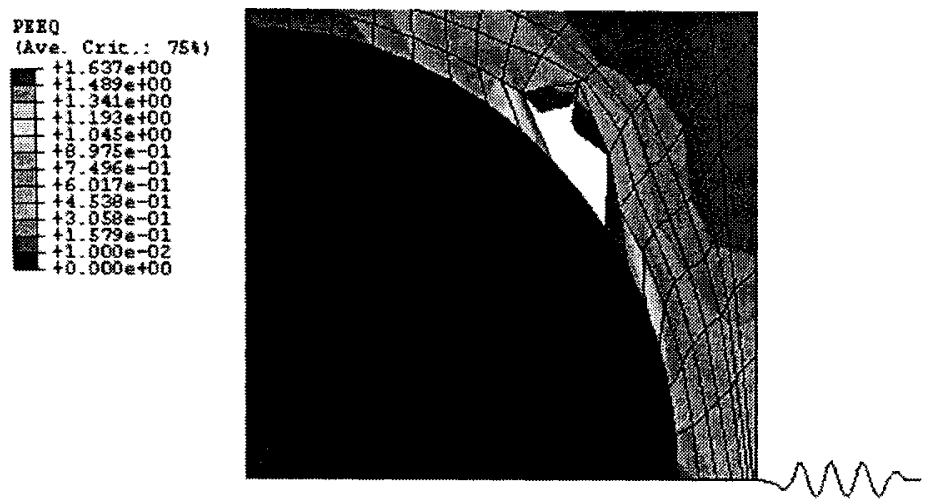

(b)
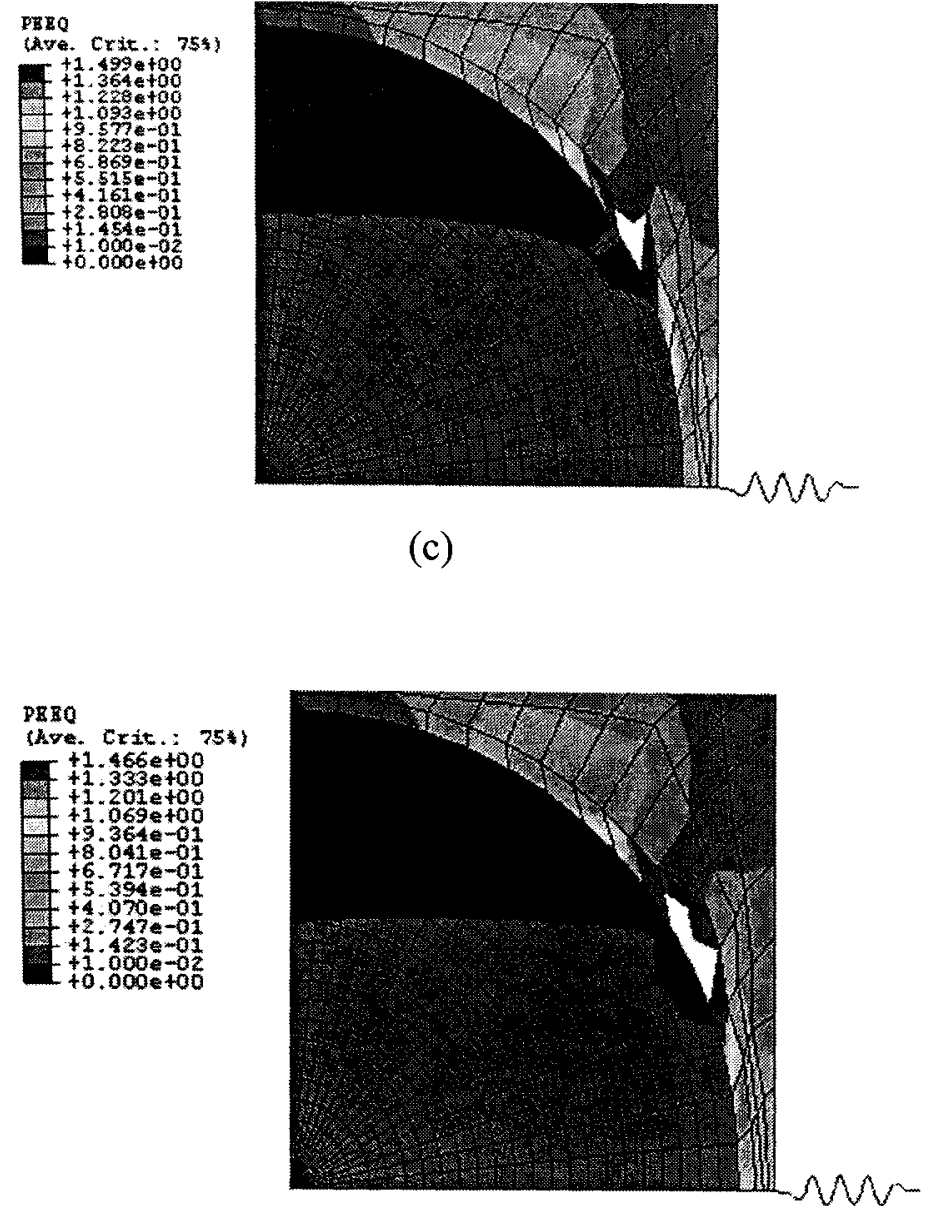

(d)

Fig. 8.7 c: Contours of plastic strain for $\mathrm{Vm}=19.7 \%$ for a) void1 at $14.2 \%$ and b) void 2 at $15.6 \%$ effective strains and $\mathrm{Vm}=34 \%$ for c) void1 at $9 \%$ d) void 2 at $10 \%$ effective strains. 


\subsection{Simulation of a Tensile Bar Test:}

The response of a tensile bar for the three $V_{m}$ considered is simulated to assess the ability of the calibrated Gurson-Tvergaard model to capture localization and fracture at the macro scale. The tensile bar is modeled using symmetry conditions, which reduces the problem to $1 / 4$ the model size. The necking is triggered at the center of the model by marginally reducing the radial dimension at that spot. The mesh is finer in the middle where necking occurs and coarsens away from the middle uniformly. The measurements on the model are done at corresponding strain gauge length $(25.4 \mathrm{~mm})$ performed experimentally, to be able to compare the model predictions to the experimental results. The model was made with 330 axisymmetric 8 node reduced integration elements and 1099 nodes.

The model predictions in terms of the computed normalized load vs. elongation are shown in Fig. 8.8 compared to representative experimental results. The plot of the tensile bar showing the necking at failure with contours of plastic strain for the three $V_{m}$ considered are shown in Fig. 8.9. The Gurson-Tvergaard model with the calibrating parameters determined in this work (see table 8.2) was used in performing these simulations.

The growth of voids and nucleation of new voids was introduced in the GursonTvergaard model by the following equation:

$$
d f_{v}=d f_{n u c l .}+d f_{g r}
$$

And the total void volume fraction will be:

$$
f_{t}=f_{0}+f_{n u c l .}+f_{g r}
$$



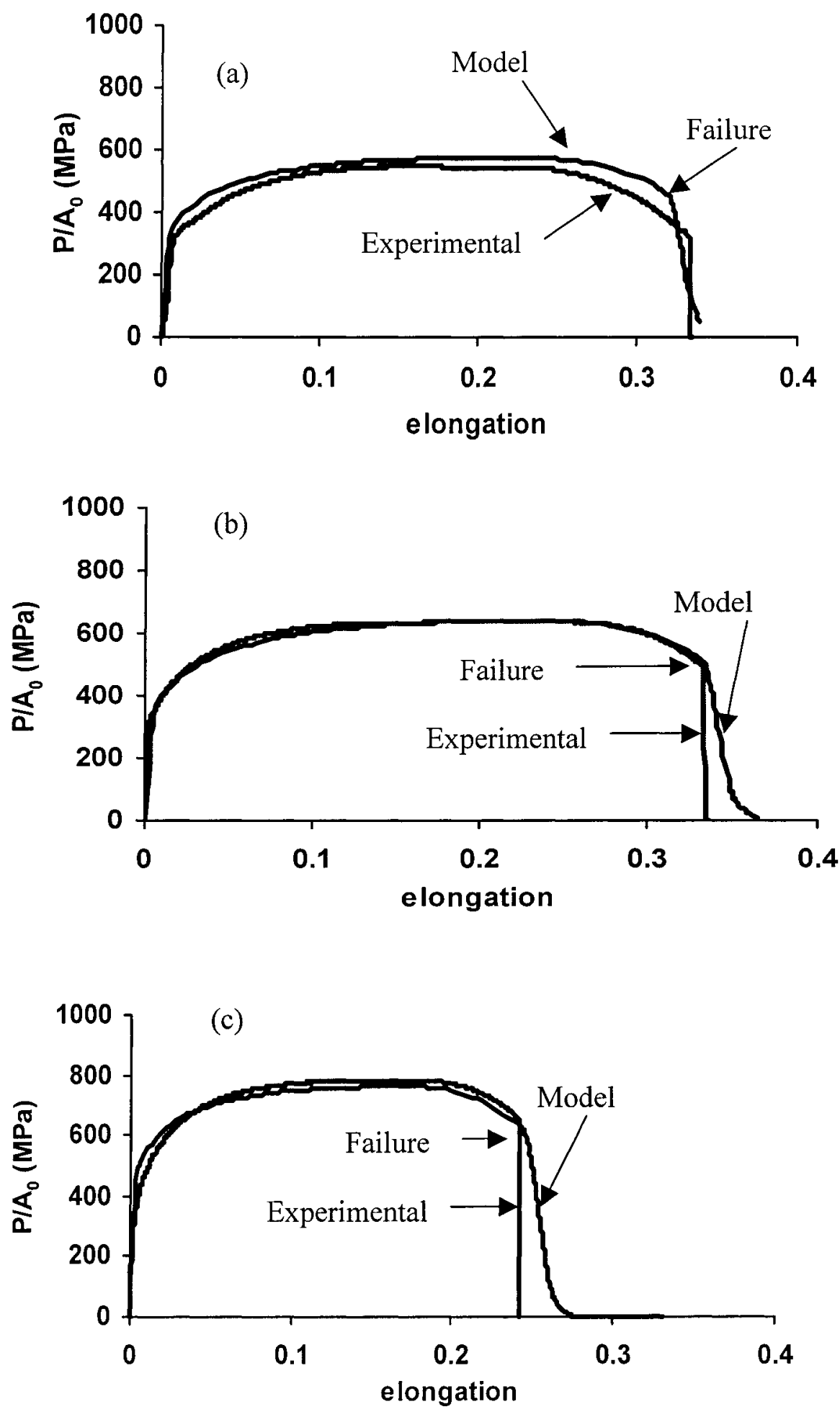

Fig. 8.8: Comparison between tensile bar model prediction and representative experimental results for a) $V_{m}=14 \%$, b) $19.7 \%$ and c) $34 \%$. 


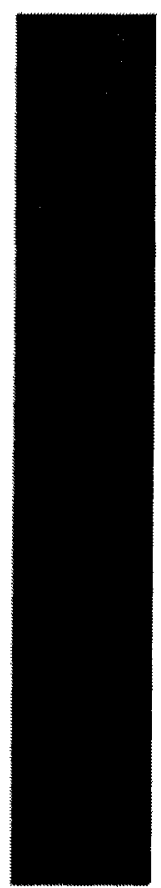

$\mathrm{PBEO}$

(kve. Crit.: 7s)

$+1.109=+00$

$-5+1.032=+0$

$4+9.555 \mathrm{e}-01$

$+8.766 \mathrm{E}-01$

$4+8.0170-01$

$-+7.248=-0$

$++6.479=01$

- +5.710 -01

$-+941 \mathrm{e}-01$

$+3.403=-01$

$+2634=01$

$+1.865=-01$

(a)

(b)
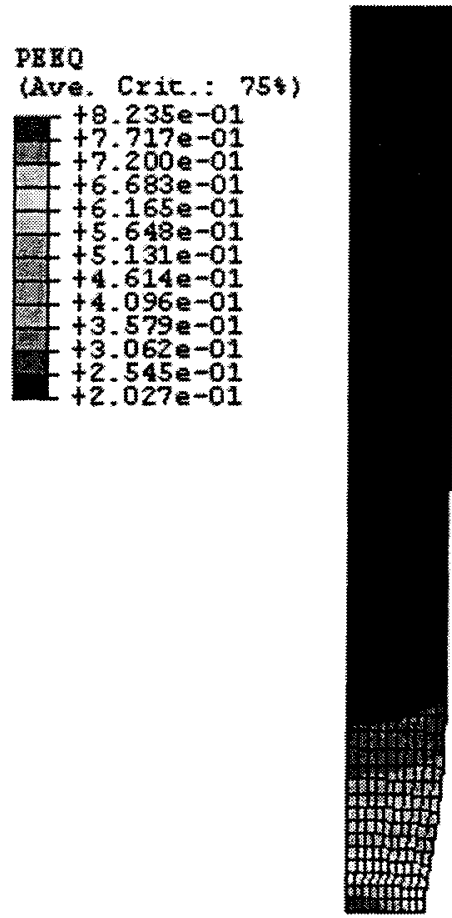

PERO

(Ave. C*16.: 75*

+1 . 261 100

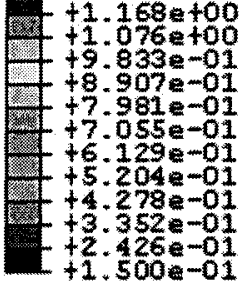

(c)

(d)

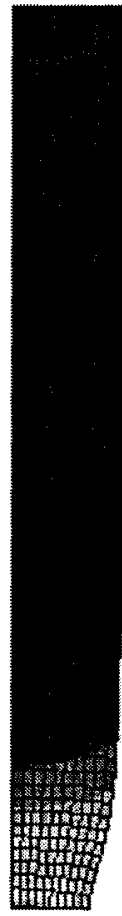

Fig. 8.9: Contours of plastic strain showing necking of the tensile bar model at fracture a) mesh design, b) $V_{m}=14 \%$, c) $V_{m}=19.7 \%$ and d) $V_{m}=34 \%$. 
In numerical modeling, the void nucleation is generally written in two parts, namely, the stress-controlled and strain-controlled quantities. Gurson (1977) and Goods and Brown (1979) have shown that the nucleation of voids could be correlated to the equivalent plastic strain. Although there are a number of workers that have reported the correlation to equivalent stress, the strain-controlled model will be used in this work. Void nucleation and void growth were defined by equations 2.24 and 2.27 , respectively, and the nucleation parameters for the strain-controlled case were defined in equation 2.25 and repeated here for convenience:

$$
A=\frac{f_{N}}{S_{N} \sqrt{2 \pi}} \exp \left[\frac{-1}{2}\left(\frac{\varepsilon_{e}^{p}-\varepsilon_{N}}{S_{N}}\right)^{2}\right]
$$

Where $f_{N}$ is the volume fraction of particles or inclusions (or volume fraction of existing voids), $\varepsilon_{N}$ is the mean nucleation strain and $S_{N}$ is the corresponding standard deviation. In this work these values are taken as, $\varepsilon_{N}=0.3, S_{N}=0.1$ (Chu and Needleman (1990)) and $f_{0}$ was taken as 0.0002 (Ishikawa et al. (2000)). The value of $f_{N}$ was taken as 0.012 (Ishikawa et al. (2000)), 0.023, and 0.023 for $V_{m}=14 \%, 19.7 \%$ and $34 \%$ respectively. At low $V_{m}$ the $f_{N}$ parameters are taken the same as what Ishikawa et al. (2000) have reported, but at intermediate and high $V_{m}$ the values are chosen to be 0.023 , since at low $V_{m}$ the deformation mechanics and mechanisms are very similar to plain carbon steels, but at intermediate and high $V_{m}$ the mechanics and mechanisms of deformation are quite different as shown in the preceding Chapters. Although the parameters work well for the model describing the fracture behavior of DP-steels, rationally it would be better to investigate the nucleation parameters for DP-steels which is beyond the scope of this work

The parameters in the Gurson-Tvergaard equation can also be determined from results given by Faleskog et al. (1998) presented in table 2.1 (Chapter 2). For the ranges of $V_{m}$ investigated in this work, the corresponding parameters can be determined by using table 
7.3 (Chapter 7.3) and table 2.1 (Chapter 2.3.2) and linearly interpolating and/or extrapolating to get the constants corresponding to the hardening of each $V_{m}$, and the yield stress to the stiffness ratio, which are shown in table 8.3. The yield strength to stiffness ratio for $V_{m}=14 \%, 19.7 \%$ and $34 \%$ are $0.001882,0.003571$ and 0.002371 respectively.

\begin{tabular}{|c|c|c|c|}
\hline & $V_{\mathrm{m}}=14 \%$ & $V_{\mathrm{m}}=19.7 \%$ & $V_{\mathrm{m}}=34 \%$ \\
\hline$q_{1}$ & 1.930 & 1.850 & 1.718 \\
\hline$q_{2}$ & 0.785 & 0.794 & 0.859 \\
\hline
\end{tabular}

Table 8.3: Calibration parameters obtained from results of Faleskog et al. (1998).

Fig. 8.10 depicts the results of the simulation of a tensile bar using the parameters from Faleskog et al. (1998), shown in table 8.3, compared to the results obtained in this work. The simulation predictions obtained by employing the calibration parameters obtained in this work and the ones determined from Faleskog et al. (1998) results are compared to the experimental findings reported in Chapter 7 and summarized in table 8.4. The ROA and the elongation at fracture are measured at the instant where there is a sudden loss of the load carrying capacity (see Fig. 8.8). As can be noticed in Fig. 8.10, the predictions of Faleskog et al. (1998) do not show this sharp drop as in the case of the results of the present work, which is closer to the experimental findings.

The model predictions based on the calibrating parameters determined in this work show good agreement with the experimental results both in terms of the ROA and the elongation at fracture. The predictions made using Faleskog et al. (1998) are good as seen from table 8.4 for $V_{m}=14 \%$ but fall largely outside the experimental range for intermediate and large $V_{m}$. 


\begin{tabular}{|c|c|c|c|c|c|c|}
\hline & \multicolumn{3}{|c|}{ Elongation to fracture \% } & \multicolumn{3}{|c|}{ Reduction of area (ROA) \% } \\
\cline { 2 - 7 } & $\begin{array}{c}\text { Current } \\
\text { work }\end{array}$ & $\begin{array}{c}\text { Faleskog } \\
\text { et al. } \\
(\mathbf{1 9 9 8})\end{array}$ & Experimental & $\begin{array}{c}\text { Current } \\
\text { work }\end{array}$ & $\begin{array}{c}\text { Faleskog } \\
\text { et al. } \\
(\mathbf{1 9 9 8})\end{array}$ & Experimental \\
\hline $\begin{array}{c}\boldsymbol{V}_{\boldsymbol{m}} \\
\mathbf{1 4 . 0 \%}\end{array}$ & 32.33 & 34.20 & $32.60-36.81$ & 66.17 & 70.70 & $67.06-71.65$ \\
\hline $\begin{array}{c}\boldsymbol{V}_{\boldsymbol{m}} \\
\mathbf{1 9 . 7 \%}\end{array}$ & 33.73 & 38.86 & $33.46-36.08$ & 54.02 & 78.30 & $53.94-55.89$ \\
\hline $\begin{array}{c}\boldsymbol{V}_{\boldsymbol{m}} \\
\mathbf{3 4 . 0} \%\end{array}$ & 24.70 & 29.29 & $22.00-24.41$ & 42.80 & 68.87 & $42.94-49.43$ \\
\hline
\end{tabular}

Table 8.4: Comparison of the predicted results and the experimental findings, ROA and elongation to fracture.

\subsection{Conclusions:}

A procedure was developed in this work to determine the calibrating parameters in the Gurson-Tvergaard model. The procedure, as explained in section 8.1, determines $q_{1}$ and $q_{2}$ and assumes $q_{3}=q^{2}$, which is shown to be an insensitive parameter. The determination of the parameters is based on employing discrete voids in the micro mechanical model developed in this work, which successfully captures the deformation behavior of DP-steels at different levels of $V_{m}$. The variables in the Gurson-Tvergaard model, $\sigma_{y}, \sigma_{e}, \sigma_{h}$ and $f_{v}$ are determined from the response of the micro mechanical model with voids (two void size cases) at each $V_{m}$. These are then used in the GursonTvergaard yield function making up two equations with two unknowns from which $q_{1}$ and $q_{2}$ are obtained. In determining the response of the micro models with voids the triaxiality in each void size case for a certain $V_{m}$ is maintained closely at the same values, and at strains corresponding to stress triaxiality $\mathrm{T}=1.2$ the variables $\sigma_{y}, \sigma_{e}, \sigma_{h}$ and $f_{v}$ are determined. 

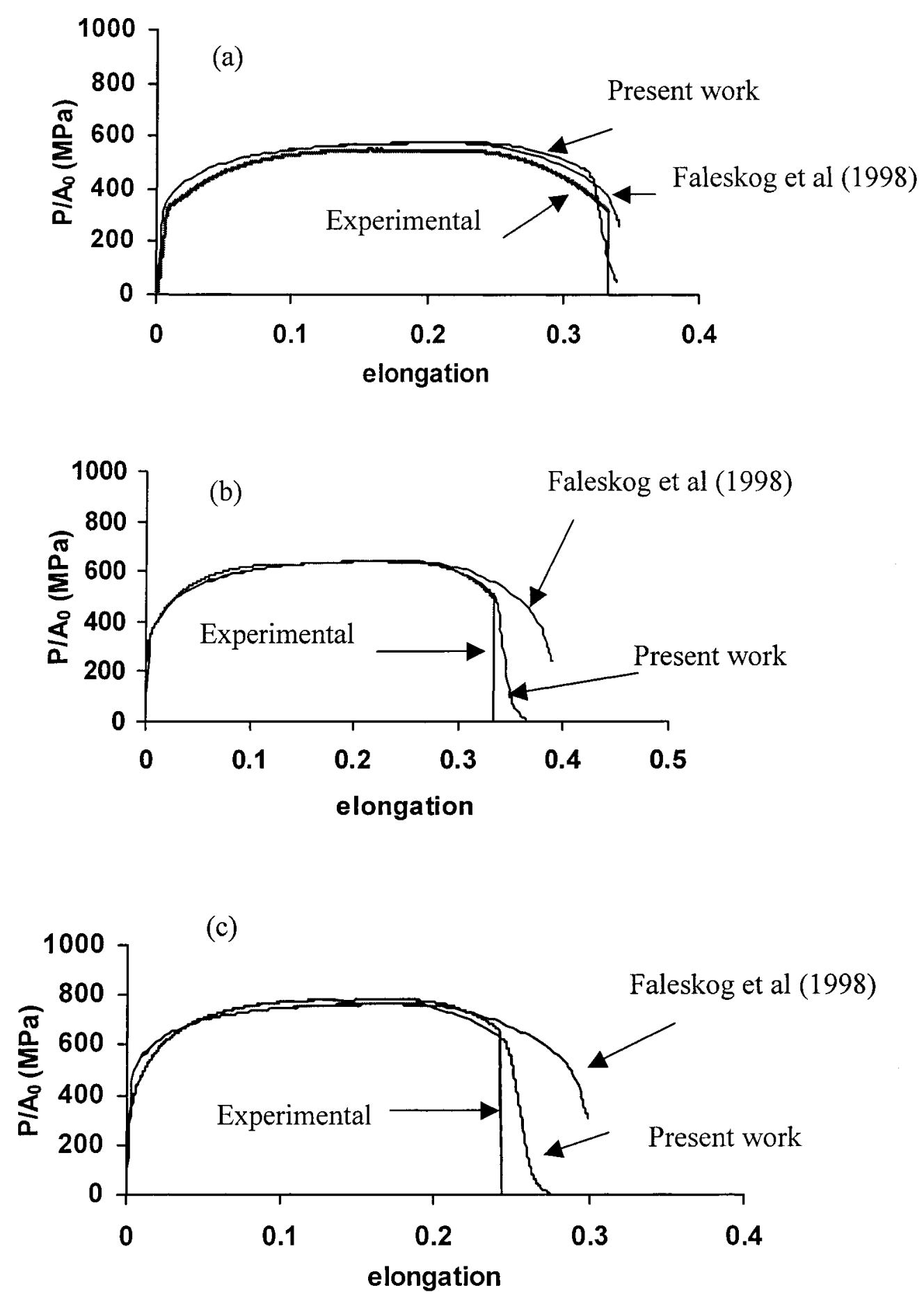

Fig. 8.10: Predicted tensile bar responses from parameters determined in this work and the ones taken from Faleskog et al. (1998) for Vm a) $14 \%$, b) $19.7 \%$ and c) $34 \%$. 
The parameters determined in this work are implemented in a finite element simulation of a tensile test bar to predict elongation at fracture and the ROA. The predicted results agree well with the experimental findings at all levels of $V_{m}$ considered, as shown in table 8.4. The calibrating parameters from Faleskog et al. (1998) show good agreement for low $V_{m}$ but at intermediate and high $V_{m}$ the results differ from the experimental range. The deformation behavior of DP-steels shows that the martensite particle does not deform plastically at $V_{m}=14 \%$ and that the deformation is not as complex as in the case of intermediate and high $V_{m}$. With this being said, the good agreement between the predicted results determined by using calibrating parameters obtained from Faleskog et al. (1998) at low $V_{m}(14 \%)$ is not surprising as at this level of $V_{m}$ the localization is not severe, similar or close to what happens in a plain carbon steel. At intermediate and high $V_{m}$, however, the deformation behavior is quite complex and is not similar to plain carbon steel, which demonstrates the need for the current model to perform the calibration of the Gurson-Tvergaard parameter. In addition, the simulation results for the tensile bar for parameters obtained from Faleskog et al. (1998) do not show a sudden drop of the load carrying capacity of the model as is experimentally observed. With the current parameters, the drop is noticeable which agrees well with experimental findings at the three levels of $V_{m}$. 


\section{CHAPTER}

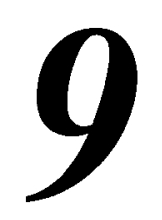

\section{SUMMARY, CONCLUSIONS AND FUTURE WORK RECOMMENDATIONS}

In this research work a micro mechanical model has been developed which can predict the essential mechanical properties of DP-steels and which can be used to understand the mechanics and mechanisms involved in the deformation process in such materials. This multi disciplinary project involves work on both metallurgy and metallography in addition to the solid mechanics, as the main concern is to characterize material properties from microstructural aspects of the material. Qualitative behavior of DP-steels has been adopted from the literature as it is comprehensively studied and reported by many workers due to their impressive mechanical properties especially for the automotive industry. Different modeling idealizations have been considered and the one, which had the intrinsic ability to capture the essential material behavior, is recommended. In the micro mechanical model, the behavior of the constituents was first adopted from the 
literature and different strengthening effects were studied numerically with confirmation from the literature to decide on the strengthening effects, which substantially affect the material behavior in order to accommodate them into the micro mechanical model. When the above was done, the constitutive material behavior of the constituents of a commercial DP-steel was developed and used with the micro mechanical model together with the material strengthening mechanisms determined by metallographical means in order to quantitatively capture the material behavior. The main findings can be summarized as follows:

Using several idealizations based on plane strain and axisymmetric models it was firstly shown that the axisymmetric model possesses the intrinsic ability to capture the essential DP-steel mechanical behavior qualitatively.

$>$ All the models considered have similar stress-strain trends at low $V_{m}$. The martensite particles undergo no measurable strain, which is in agreement with experimental observation by Shen et al. (1986). At high $V_{m}$, the martensite particles in the plane strain models behave elastically while they plastically deform in the axisymmetric case.

Although, the plastic deformation that takes place in the martensite particles is small, it is believed to be an important phenomenon, which alters the mechanics of deformation of the aggregate significantly as shown in Fig. 5.11 in Chapter 5. The inability of plane strain models to show any plastic deformation in the martensite particles is believed to be the main reason behind the suppression of the localization behavior they display. In the plane strain idealization, the martensite particles are geometrically assumed to be long cylinders in a ferrite matrix, rather than a spherical particle in a cylindrical matrix. As a result, for the same volume fraction, the area fraction of martensite is larger in the axisymmetric model, causing yield to occur at lower strains, which more closely resembles the actual material response. 
Different strengthening mechanisms have been investigated and the ones that have been shown to affect the material behavior significantly were accounted for in the axisymmetric model.

The effect of the particle size distribution is studied and has been shown to have considerable influence on the behavior of the composite. Different particle size ratios have been examined to investigate the size ratio effect on the micro mechanical model to evaluate its importance in prediction of the response of the material. Examination of the results show that at low $V_{m}$ and particle size ratio of $\mathrm{R}=1 / 2$ there is no measurable difference in the response compared to the model with $\mathrm{R}=1$. At high $V_{m}$ the effect is much more pronounced. In addition, the difference comes about only when the larger martensite particle undergoes plastic deformation and this occurs at the larger particle only as shown in Fig. 6.7b in Chapter 6. The onset of plastic deformation of the larger martensite particle in the $\mathrm{R}=1 / 2$ model occurs at lower $V_{m}$ than the particles in the $\mathrm{R}=1$ model. This takes place due to the fact that the ferrite phase surrounding the larger particle is very constrained compared to the ferrite in the latter case at the same $V_{m}$, which forces the larger martensite particle to undergo earlier plastic deformation.

In agreement with the experimental observations, the model with two particle sizes shows two different mechanisms of deformation occurring in the martensite particles. The larger particle neighboring the highly strained ferrite matrix, which Shen et al. (1986) considered as weak martensite particles, undergo plastic deformation at intermediate and high $V_{m}$, while the smaller particle experiences no plastic deformation. In other words, the larger particle shows a duplex deformation mechanism while the smaller martensite particle shows only a particle strengthening mechanism. 
Martensite dilution has been investigated numerically and found to have significant effect on the mechanics and mechanism of deformation of the DPsteels. This effect has been implemented in the model developed in this work.

Grain size effect on the mechanical behavior has been shown to have significant influence on the behavior of DP-steels. For this effect results from the literature have been used to show the capability of the model to capture the effect of grain size effect for several levels of ferrite grain sizes.

Quantitative comparison between the model predictions and the experimental results demonstrate the ability of the model to capture the mechanical behavior of DP-steels at low, intermediate and high $V_{m}$. The model can capture the important mechanical properties such as the yield strength, uniform elongation, UTS and the strain-hardening rate. The model is also shown to capture the mechanism of deformation of DP-steels at the different levels of $V_{m}$.

DP-steels have been shown to fail in a ductile manner by void nucleation, growth and coalescence. For a void containing material, the most widely known material damage model is the Gurson-Tvergaard model, which has been used comprehensively in the literature. This model has some calibrating parameters, which need to be determined. Faleskog et al. (1998) and Geo et al. (1998) presented a procedure for choosing the proper calibration parameters $\left(q_{1}\right.$ and $\left.q_{2}\right)$ for different strain hardening rates of metallic materials using a three dimensional model for moderate and high strain hardening materials as the parameters suggested by Tvergaard $(1981,1982)$ and Tvergaard and Needleman (1984) were not adequately capturing the real material behavior at different strain hardening rates. Although the model they used captures failure occurring in plain carbon steels, they do not represent MP-steels.

The mechanics and mechanisms of deformation of DP-steels, as shown in this work, are different from plain carbon steels. Unlike the plain carbon steels, DP- 
steels undergo a series of mechanisms while deforming that introduces different strain localizations between voids than the plain carbon steels, which should be considered when parameter calibration is considered. This demonstrates the need for calibrating the Gurson-Tvergaard model using the micro model developed in this work, which well captures the mechanics and mechanisms of deformation taking place in DP-steels.

A procedure for determination of the calibrating parameters in the GursonTvergaard model has been developed in this work. The procedure involves employing discrete voids in the micro mechanical model at the martensite-ferrite interface, following the findings of the overwhelming reports, which show that the failure of DP-steels occurs due to martensite-ferrite interface decohesion.

The procedure is shown to adequately produce the appropriate calibrating parameters, which is validated by introducing them in a finite element simulation of a tensile bar and comparing the predictions with the experimental failure results such as the elongation at fracture and the reduction of area at fracture.

\section{Original Contribution to the Literature:}

A micro mechanical model has been developed to characterize the deformation behavior of DP-steels consisting of martensite dispersed in a matrix of ferrite. In addition to accounting for the $V_{m}$, this model accounts for grain size, carbon dilution, which causes martensite softening and particle size distribution, which are uniquely developed in this work.

To predict the failure behavior of DP-steels, the deformation behavior of the DPsteels predicted by the micro mechanical model mentioned above, is coupled with 
the Gurson-Tvergaard model which is used widely to predict ductile failure of materials by void nucleation, growth and coalescence. The calibrating parameters in the Gurson-Tvergaard yield function are determined uniquely in a procedure developed in this work, which accounts for the void volume fraction and the stress triaxiality by employing discrete voids in the micro mechanical model.

The current work presents a predictive micro mechanical model and a calibrating procedure, which can be used to characterize DP-steels to obtain their deformation and fracture behavior at various levels of $V_{m}$ and at various chemistries, which minimizes the extent of expensive experimental investigation. The only requirement is to specify the material chemistry and the softer phase behavior, which can easily be obtained as shown in the method developed and used in this work.

This research work has resulted in the following contributions to the literature:

1. Al-Abbasi F. M. and Nemes J. A., 2003. Micro Mechanical Modeling of the Effect of Particle Size Difference in Dual Phase Steels, International Journal of Solids and Structures, Vol. 40, pp. 3379-3391.

2. Al-Abbasi F. M. and Nemes J. A., 2003. Micro Mechanical Modeling of Dual Phase Steels, International Journal of Mechanical Sciences, Vol. 45 (9), pp. 14491465.

3. Al-Abbasi, F. M. and Nemes, J. A., 2003. Experimental and Finite Element Modeling for Prediction of Optimum Phase Combinations in Dual Phase Steels, International Conference on Processing and Manufacturing of Advanced Materials, Spain, Madrid, July 7 - 11, part 2, pp. 1559-1564.

4. Nemes, J. A. and Al-Abbasi, F. M., 2003. Micro Mechanical Models for Optimization of Constituent Volume Fraction in Dual Phase Steels, 9th International Conference on the Mechanical Behavior of Materials, Geneva, Switzerland, May 25-29, CD-Rom (6 pages). 
5. Al-Abbasi, F. M. and Nemes, J. A, 2001. Micro Mechanical Modeling of Dual Phase Steels, A Conference Presentation in: Mechanics and Materials, ASME materials and Applied Mechanics Divisions, June 27-29.

\section{Future Work Recommendations:}

1) Use the model developed in this work to investigate the high rate deformation behavior of DP-steels. The micromechanical model response under high rate conditions can be achieved by applying displacement boundary condition at high speed or velocity. This will allow the characterization of DP-steels for high rate processes such as cold heading of fasteners. In order to investigate the high rate response of the micromechanical model, the high rate behavior of the constituents will be required which is thus far possible. When this is achieved, the need for the mechanical testing to investigate the aggregate high rate response will be reduced drastically. The material response from the early deformation stages to failure can be worked out in the same way the characterization of DP-steel for the quasi-static conditions in the current work was performed but for the high rate deformation conditions. This indeed holds great merits, as it will affect almost all the manufacturing processes that operate at high rate.

2) Extend this model to capture the behavior of multiphase steels such as TRIP steels that consist of austenite and martensite in a matrix of ferrite, which display very attractive mechanical properties. Other types of steel phases can also be considered such as bainitic and pearlitic steels. 


\section{References:}

Abdalla A.J., Hein L.R.O., Pereira M.S. and Hashimoto T.M., 1999. Mechanical Behavior of Strain Aged Dual Phase Steels, Materials Science and Technology, Vol. 15, pp. 1167-1170.

Aboudi J. and Arnold S.M., 2000. Micromechanical Modeling of the Finite Deformation of Thermoelastic Multiphase Composites, Mathematics and Mechanics of Solids, Vol. 5, pp. 75-99.

Aboudi J., 1989. Micromechanical Analysis of Composites by Method of Cells, Applied Mechanics Reviews, Vol. 42 (7), pp. 193-221.

Aboudi J., 1991. Mechanics of Composite Materials - A Unified Micromechanical Approach, Elsevier, Amsterdam.

Ahmed E., Tanvir M., Kanwar L.A. and Akhter J.I., 2000. Effect of Microvoid Formation on the Tensile Properties of Dual-Phase Steel, Journal of Materials Engineering and Performance, Vol. 9 (3), pp. 306-310.

Anderson T.L., 1995. Fracture Mechanics: Fundamentals and Applications, $2^{\text {nd }}$ Edition, CRC Press.

Argon A.S., IM J. and Safoglu R., 1975. Cavity Formation from Inclusions in Ductile Fracture, Metallurgical Transactions, Vol. 6 (A), pp. 825-837.

Askeland D.R., 1985. The Science and Engineering of Materials, PWA publishers, Alternate Edition.

ASM International, 1992. Tensile Testing, Materials Park, OH, USA.

ASM International, 2000. Practical Guide to Image Analysis, Materials Park, OH, USA.

ASM, 1985. Metals Handbook, $9^{\text {th }}$ Edition, Vol. 9, Metallography and Microstructures, Metals Park, Ohio.

ASM, 1990. Metals Handbook, $10^{\text {th }}$ Edition, Vol. 1, Properties and Selection: Iron and Steels, Metals Park, Ohio.

ASTM E 112-96, 1998. Mechanical Testing, Elevated and Low Temperature Tests; Metallography, Standard Test Method for Determining Average Grain Size, Vol. 3.01, Section 3, pp. 237-259. 
ASTM E 1245-95, 1998. Mechanical Testing, Elevated and Low Temperature Tests; Metallography, Standard Test Method for Determining the Inclusion or Second-Phase, Constituent Content of Metals by Automatic Image Analysis, Vol. 3.01, Section 3, pp. 237-259.

ASTM E 562-95, 1998. Mechanical Testing, Elevated and Low Temperature Tests; Metallography, Standard Test Method for Determining Volume Fraction by Systematic Manual Point Count, Vol. 3.01, Section 3, pp. 522-527.

Bag A., Ray K.K. and Dwarakadasa E.S., 1999. Influence of Martensite Content and Morphology on Tensile and Impact Properties of High-Martensite Dual-Phase Steels, Metallurgical and Materials Transactions, Vol. 30 (A), pp. 1193-1202.

Balliger N.K. and Gladman T., 1981. Work Hardening of Dual-Phase Steels, Metal Science, Vol. 15 (3), pp. 95-108.

Balliger N.K., 1982. Advances in the Physical Metallurgy and Applications of Steels, Book 284, The Metals Society, London, pp. 73-83.

Baxter S.C. and Graham L.L., 2000. Development of Micromechanics Based Material Property Fields for Random Composites, Proceeding of the $8^{\text {th }}$ ASCE Specialty Conference on Probabilistic Mechanics and Structural Reliability, Notre Dame, paper 204.

Becker R., Smelser R.E., Richmond O. and Appleby E.J., 1989. The Effect of Void Shape on Void Growth and Ductility in Axisymmetric Tension Tests, Metallurgical Transactions, Vol. 20 (A), pp. 853-861.

Bensoussan A., Lions J.L. and Papanicolaou G., 1978. Asymptotic Analysis for Periodic Structures, Elsevier North-Holland INC, New York.

Beremin F.M., 1981. Cavity Formation from Inclusions in Ductile Fracture of A508 Steel, Metallurgical Transactions, Vol. 12 (A), pp. 723-731.

Biggs W.D., 1960. The Brittle Fracture of Steel, Macdonald and Evans LTD, London.

Bourell D.L. and Rizk A., 1983. Influence of Martensite Transformation Strain on the Ductility of Dual Phase Steels, Acta Metallurgica, Vol. 31, pp. 609 - 617.

Bridgman P.W., 1952. Studies in Large Plastic Flow and Fracture, New York, McGrawHill.

Bridgman P.W., 1944. The Stress Distribution at the Neck of a Tension Specimen, Transactions of the ASM, Vol. 32, pp. 553-572. 
Brown L.M. and Stobbs W.M., 1976. The Work-Hardening of Copper Silica v. Equilibrium Plastic Relaxation by Secondary Dislocations, Philosophical Magazine, Vol. 34, pp. 351-372.

Byun T.S. and Kim I.S., 1993. Tensile Properties and Inhomogeneous Deformation of Ferrite-Martensite Dual-Phase Steels, Journal of Materials Science, Vol. 28, pp. 29232932.

Cahn R.W. and Haasen P., 1996. Physical Metallurgy, Vol. II, $4^{\text {th }}$ Edition, Elsevier Science B.V., Amsterdam.

Chang P.H. and Preban A.G., 1985. The Effect of Ferrite Grain Size and Martensite Volume Fraction on the Tensile Properties of Dual Phase Steel, Acta Metallurgica, Vol. 33 (5), pp. 897-903.

Christman T., Needleman A. and Suresh S., 1989. An Experimental and Numerical Study of Deformation in Metal-Ceramic Composites, Acta Materialia, Vol. 37, No. 11, pp. 3029-3050.

Clinton J.M. and Kelly P.M, 1968. The Strength of Ferrous Martensite, Acta Metallurgica, Vol. 16, pp. 637-656.

Cockcroft M.G., 1967. Ductility, Ductile Fracture in Cold Working Operations, Papers presented at a Seminar of the American Society for Metals, Oct. 14 and Oct. 15, Metals park, Ohio, USA.

Danielsson M., Parks D.M. and Boyce M.C., 2002. Three-Dimensional Micromechanical Modeling of Voided Polymeric Materials, Journal of the Mechanics and Physics of Solids, Vol. 50, pp. 351-379.

Davies R.G., 1978a. The Deformation Behavior of a Vanadium-Strengthened Dual Phase Steel, Metallurgical Transactions, Vol. 9 (A), pp. 41-52.

Davies R.G., 1978b. The Mechanical Properties of Zero-Carbon Ferrite-Plus-Martensite Structures, Metallurgical Transactions, Vol. 9 (A), pp. 451-455.

Davies R.G., 1978c. Influence of Martensite Composition and Content on the Properties of Dual Phase Steels, Metallurgical Transactions, Vol. 9 (A), pp. 671-679.

Davis R.O. and Selvadurai A.P.S., 2002. Plasticity and Geomechanics, Cambridge University Press, UK.

DeHoff R.T. and Rhines F.N., 1968. Quantitative Microscopy, McGraw-Hill Book Company, USA. 
Dieter E.G., 1967. Ductility, Introduction to Ductility, Papers presented at a Seminar of the American Society for Metals, Oct. 14 and Oct. 15, Metals Park, Ohio, USA.

Faleskog J., Geo X. and Shih F., 1998. Cell Model for Nonlinear Fracture Analysis-I, International Journal of Fracture, Vol. 89, pp. 355-373.

Faleskog J. and Shih C.F., 1997. Micromechanics of Coalescence-I. Synergistic Effects of Elasticity, Plastic Yielding and Multi-Size-Scale Voids, Journal of the Mechanics and Physics of Solids, Vol. 45 (1), pp. 21-50.

Frechette, V.D., 1990. Failure Analysis of Brittle Materials, American Ceramic Society, Westerville, Ohio.

Garrison W.M. and Moody N.R., 1987. Ductile Fracture, Journal of the Physics and Chemistry of Solids, Vol. 48, pp. 1035-1074.

Gdoutos E.E., 1993. Fracture Mechanics, Edited by Gladwell G.M.L., Kluwers Academic Publishers, Netherlands.

Geo X., Faleskog J. and Shih C.F., 1998. Cell Model for Nonlinear Fracture Analysis-II, Fracture-Process Calibration and Verification, International Journal of Fracture, Vol. 89, pp. 375-398.

Geo X., Faleskog J. and Shih C.F., 1998. Ductile Tearing in Part-Through Cracks: Experimental and Cell-Model Predictions, Engineering Fracture Mechanics, Vol. 59 (6), pp. 761-777.

Gerbase J., Embury J.D. and Hobbs R.M., 1979. The Mechanical Behavior of Some Dual Phase Steels, Structure and Properties of Dual-Phase Steels, Edited by Kot R.A. and Morris J.W., TMS-AIME, New York, 118-143.

Gladman T., 1997. The Physical Metallurgy of Microalloyed Steels, The Institute of Materials, The University Press, Cambridge, London.

Gladman T., Mclvor I.D. and Pickering F.B., 1972. Some Aspects of the StructureProperty Relationships in High-Carbon Ferrite-Pearlite Steels, Journal of the Iron and Steel Institute, Vol. 210, pp. 916-930.

Goldestein J.I., Newbury D.E., Echlin P., Joy D.C., Fiori C., and Lifshin E., 1981. Scanning Electron Microscopy and X-ray Microanalysis, Plenum Press, New York.

Goods S.H. and Brown L.M., 1979. The Nucleation of Cavities by Plastic Deformation, Acta Metallurgica, Vol. 27. pp. 1-15. 
Graham L.L. and Baxter S.C., 2000. Stochastic Finite Element Analysis Based on Micromechanics of Random Composite Materials, Proceeding of the $8^{\text {th }}$ ASCE Specialty Conference on Probabilistic Mechanics and Structural Reliability, Notre Dame, paper 203.

Gurson A.L., 1977. Continuum Theory of Ductile Rupture by Void Nucleation and Growth: Part I - Yield Criteria and Flow Rules for Porous Ductile Media, Journal of Engineering Materials and Technology, Vol. 99, pp. 2-15.

Hall E.O., 1951. The deformation and Aging of Mild Steel: III Discussion of Results. Proceedings of the Physical Society of London., Vol. 64 (B), pp. 747-753.

Hill R., 1950. The Mathematical Theory of Plasticity, New York, Oxford University Press.

Hill R., 1963. Elastic Properties of Reinforced Solids: Some Theoretical Principles, Journal of the Mechanics and Physics of Solids, Vol. 11, pp. 357-372.

Honeycombe R.W.K., 1990. Steels: Microstructure and Properties, E. Arnold, London, 1990.

Hosford W.F. and Caddell R.M., 1993. Metal Forming, Mechanics and Metallurgy, by Prentice Hall, $2^{\text {nd }}$ Edition, Upper Saddle River, USA.

Huang Y. and Kinloch A.J., 1992. Modeling of the Toughening Mechanisms in the Rubber-Modified Epoxy Polymers: Part I -Finite Element Analysis Studies, Journal of Materials Science, Vol. 27, pp. 2753-2762.

Huper T., Endo S., Ishikawa N. and Osawa K., 1999. Effect of Volume Fraction of Constituents Phases on the Stress Relationship of Dual Phase Steel. Iron and Steel Institute of Japan International, Vol. 39, pp. 288-294.

Ishikawa N., Parks D.M., Socrate S. and Kurihara M., 2000. Micromechanical Modeling of Ferrite-Pearlite Using Finite Element Unit Cell Models, Iron and Steel Institute of Japan International, Vol. 40 (11), pp. 1170-1179.

Jeulin D. and Ostoja-Starzewski M., 2001. Mechanics of Random and Multiscale Microstructures, International Centre for Mechanical Sciences, courses and lectures No. 430, Springer-Verlag Wien, New York.

Jiang M., Jasiuk I. and Ostoja-Starzewski M, 2002. Apparent Elastic and Elastoplastic Behavior of Periodic Composites, International Journal of Solids and Structures, Vol. 39 (1), pp. 199-212. 
Jiang M., Ostoja-Starzewski M. and Jasiuk I., 2001. Scale-Dependent Bounds on Effective Elastoplastic Response of Random Composites, Journal of the Mechanics and Physics of Solids, Vol. 49, pp. 665-673.

Jiang Z., Guan Z. and Lian J., 1993. The Relationship Between Ductility and Material Parameters for Dual Phase Steel, Journal of Materials Science, Vol. 28, pp. 1814-1818.

Kang S., Kwon H., 1987. Fracture Behavior of Intercritically Treated Complex Structure in Medium-Carbon 6 Ni Steel, Metallurgical Transactions, Vol. 18 (A), pp. 1587-1592.

Kelly P. M. and Nutting J., 1965. Strengthening Mechanisms in Martensite, Physical Properties of Martensite and Bainite, Special Report No. 93, The Iron and Steel Institute, pp. 166-170.

Kim H.S., Hong S.I. and Kim S.J, 2001. On the Rule of Mixtures for Predicting the Mechanical Properties of Composites with Homogeneously Distributed Soft and Hard Particles, Journal of Materials Processing and Technology, Vol. 112, pp. 109-113.

Kim N.J. and Thomas G., 1981. Effect of Morphology on the Mechanical Behavior of Dual Phase Fe / 2Si / 0.1C Steel, Metallurgical Transactions, Vol. 12 (A), pp. 483-488.

Knott J.F., 1980. Micromechanisms of Fibrous Crack Extension in Engineering Alloys, Metal Science, Vol. 14, pp. 327-336

Knott J.F., 1989. Effects of Microstructure and Stress-State on Ductile Fracture in Metallic Alloys, Advances in Fracture Research, Proceeding of the $7^{\text {th }}$ International Conference on Fracture (ICF7), Pergammon Press, Oxford, UK, pp. 125-138.

Koo J.Y. and Thomas G., 1977. Design of Duplex Low Carbon Steels for Improved Strength: Weight Applications. Formable HSLA and Dual Phase Steels, Edited by Davenport A.T., AIME, New York, pp. 40-55.

Korzekwa D.A., Lawson R.D., Matlock D.K. and Krauss G., 1980. A Consideration of Models Describing the Strength and Ductility of Dual-Phase Steels, Scripta Metallurgica, Vol. 14, pp. 1023-1028.

Krauss G. and Marder R.A., 1971. The Morphology of Martensite in Iron Alloys, Metallurgical Transactions, Vol. 2, pp. 2343-2357.

Krauss G., 1977. Martensitic Transformation, Structure and Properties, Hardenability Concepts With Applications to Steel, Edited by Done D.V. and Kirkaldy J.S., Chicago , USA, October 24-26, pp. 229-246.

Leslie W.C. and Sober R.J., 1967. The Strength of Ferrite and of Martensite as Functions of Composition, Temperature and Strain Rate, Transactions of the ASM, Vol. 60, pp. 459484. 
Leslie W.C., 1981. The Physical Metallurgy of Steels, McGraw-Hill, p. 217.

Liedl U., Traint S. and Werner E.A., 2002. An Unexpected Feature of the Stress-Strain Diagram of Dual Phase Steel, Computational Materials Science, Vol. 25, pp. 122-128.

Llorca J., Needleman A. and Suresh S., 1991. An Analysis of the Effects of Matrix Void Growth on Deformation and Ductility in Metal-Ceramic Composite, Acta Metallurgica et Materialia, Vol. 39, No. 10, pp. 2317-2335.

Lubliner J., 1990. Plasticity Theory, Macmillan Inc., USA.

Marder R.A. and Krauss G., 1970. The Effect of Morphology on the Strength of Lath Martensite, Proceedings of the $2^{\text {nd }}$ International Conference on the Strength of Metal Alloys, ASM, pp. 822-823.

Maynier P., Jungmann B. and Dollet J., 1977. Creusot-Loire System for the Prediction of the Mechanical Properties of Low Alloy Steel Products, Hardenability Concepts With Applications to Steel, Edited by Done D.V. and Kirkaldy J.S., Chicago, USA, October 24-26, pp. 518-545.

McClintock F.A., 1968. A Criteria for Ductile Fracture by Growth of Holes, Journal of Applied Mechanics, Vol. 35, pp. 363-371.

Modin H. and Modin S., 1973. Metallurgical Microscopy, Butterworth \& Co. Ltd, London, UK.

Mori T. and Tanaka K., 1973. Average Stress in Matrix and Average Elastic Energy of Materials With Misfitting Inclusions, Acta Metallurgica,Vol. 21, pp. 571-574.

Morris J.W., Krenn C.R. and Gue Z., 1999. Heat Treating: Including Steel Heat Treating in the New Millennium. Proceedings of the $19^{\text {th }}$ Conference of an International Symposium in Honor of Professor George Krauss, Edited by Midea S.J. and Pfaffman G.D., 1- 4 November, pp. 526-535.

Morrison W.B., 1966. The Effect of Grain Size on the Stress-Strain Relationship in LowCarbon Steel, Transactions of the ASM, Vol. 59, pp. 824-846.

Nam W.J. and Bae C.M., 1999. Microstructure Evolution and its Relation to Mechanical Properties in a Drawn Dual-Phase Steel, Journal of Material Science, Vol. 34, pp. 56615668.

Orsini V.C. and Zikry M.A., 2001. Void Growth and Interaction in Crystalline Materials, International Journal of Plasticity, Vol. 17, pp. 1393-1417. 
Ostoja-Starzewski M., 2000. A Universal Material Property in Elasticity of Planar Random Microstructures, Proceeding of the $8^{\text {th }}$ ASCE Specialty Conference on Probabilistic Mechanics and Structural Reliability, Notre Dame, paper 201.

Ostoja-Starzewski M., 1993. Micromechanics as a Basis of Stochastic Finite Element and Differences: an overview, Applied Mechanics Reviews, Vol. 46, No. 11, pt. 2, pp. 136-147.

Ostoja-Starzewski M., 1994. Micromechanics as a basis of continuum random fields, Applied Mechanics reviews, Vol. 47, No. 1, pt. 2, pp. S221-S230.

Ostoja-Starzewski M., 1999. Microstructural Disorder, Mesoscale Finite Elements and Macroscopic Response, The Royal Society, Vol. 445, pp. 3180-3199.

Ostrom P., 1981. Deformation Models for Two-Phase Materials, Metallurgical Transactions, Vol. 12 (A), pp. 355-357.

Padmanabhan K.A., Vasin R.A. and Enikeev F.U., 2001. Superplastic Flow: Phenomenology and Mechanics, Springer-Verlag, Berlin.

Pardoen T. and Hutchinson J.W., 2000. An Extended Model for Void Growth and Coalescence, Journal of the Mechanics and Physics of Solids, Vol. 48, pp. 2467-2512.

Petch N.J., 1953. The Cleavage Strength of Polycrystals, Journal of the Iron and Steel Institute, Vol. 174, pp. 25-28.

Pickering F.B. and Gladman T., 1963. An Investigation into Some Factors which Control the Strength of Carbon Steels, Iron and Steel Institute, Special report No. 81, pp. 10-20.

Pickering F.B., 1978. Physical Metallurgy and the Design of Steels, Kelly F.R.S., Materials Science Series, Applied Science Publishers LTD, London.

Porter D.A. and Easterling K.E., 1992. Phase Transformations in Metals and Alloys, $2^{\text {nd }}$ Edition, Chapman \& Hall, London.

Ramos F.L., Matlock D.K. and Krauss G., 1979. On the Deformation Behavior of DualPhase Steels, Metallurgical Transactions, Vol. 10 (A), pp. 259-263.

Rashid M.S. and Cprek E.R., 1978. Relationship Between Microstructure and Formability in Two High-Strength, Low-Alloy Steels, Formability Topics - Metallic Materials, ASTM STP 647, American Society for Testing and Materials, Philadelphia, Pa, pp. 174190. 
Rashid M.S., 1976. GM 980X-A Unique High Strength Sheet Steel With Superior Formability, Paper 760206, Soc. Auto. Eng. Cong., Detroit, pp. 938-949.

Rice J.R. and Tracey D.M., 1969. On the Ductile Enlargement of Voids in Triaxial Stress Fields, Journal of the Mechanics and Physics of Solids, Vol. 17, pp. 201-217.

Roberts M.J. and Owen W.S., 1965. Solid Solution Hardening by Carbon and Nitrogen, Physical Properties of Martensite and Bainite, Special Report No. 93, The Iron and Steel Institute, pp. 171-178.

Roberts M.J., 1970. Effect of Transformation Substructure on Strength and Toughness of Fe-Mn Alloys, Metallurgical Transactions, Vol. 1, pp. 3287-3294.

Rogers H.C., 1967. Ductility, The Effect of Material Variables on Ductility, Papers presented at a seminar of the American Society for Metals, Oct. 14-15, Metals Park, Ohio, USA.

Rudiono and Tomota Y., 1997. Application of the Secant Method to Prediction of Flow Curves in Multi-Microstructure Steels, Acta Metallurgica, Vol. 45 (5), pp. 1923-1929.

Schaarwachter W., Ludering H. and Numann F.K., 1963, Arch. Eisebhuttenwes, Vol. 34, pp. 187-194, cited by Metals handbook, $9^{\text {th }}$ Edition, Vol. 9, Metallography and Microstructures, ASM, Metals Park, Ohio, 1985.

Shehata M.T. and Crawley A.F., 1983. Microstructure-Property Relationship in C-Mn Dual-Phase Steels, Canadian Metallurgical Quarterly, Vol. 22, No. 2, pp. 181-191.

Shen H.P., Lei T.C. and Liu J.Z., 1986. Microscopic Deformation Behavior of Martensitic-Ferritic Dual-Phase Steels, Materials Science and Technology, Vol. 2, pp. 2833.

Shen Y.L., Finot M., Needleman A. and Suresh, S., 1994. Effective Elastic Response of Two-Phase Composites, Acta Metallurgica et Materialia, Vol. 42, No. 1, pp. 77-97.

Shen Y.L., Finot M., Needleman A. and Suresh, S., 1995. Effective Plastic Response of Two-Phase Composites, Acta Metallurgica et Materialia, Vol. 43, No. 4, pp. 1701-1722.

Sinha A.K., 1989. Ferrous Physical Metallurgy, Butterworth Publishers, Stoneham, MA.

Sluis O.V.D., Schreurs P.J.G., Brekelmans W.A.M. and Meijer H.E.H, 2000. Overall Behavior of Heterogeneous Elastoplastic Materials: Effect of Microstructure Modeling, Mechanics of Materials, Vol. 32, pp. 449-462. 
Socrate S. and Boyce M.C., 2000. Micromechanics of Toughened Polycarbonate, Journal of the Mechanics and Physics of Solids, Vol. 48, pp. 233-273.

Socrate S., Boyce M.C. and Lazzeri A., 2001. A Micro Mechanical Model for Multiple Crazing in High Impact Polystyrene, Mechanics of Materials, Vol. 33, pp. 155-175.

Speich G.R. and Miller R.L., 1979. Mechanical Properties of Ferrite-Martensite Steels, Structure and Properties of Dual-Phase Steels, Edited by Kot R.A. and Morris J.W., TMS-AIME, New York, pp. 145-182.

Spiech G.R. and Warlimont H., 1968. Yield Strength and Transformation Substructure of Low-Carbon Martensite, Journal of the Iron and Steel Institute, Vol. 206, pp. 385-392.

Steinbrunner D.L. and Krauss G., 1988. Void Formation during Tensile Testing of Dual Phase Steels, Metallurgical Transactions, Vol. 9 (A), pp. 579-589.

Stone R.H., Cox T.B., Low J.R., Jr, Psioda J.A., 1985. Microstructural Aspects of Fracture by Dimpled Rupture, International Metals Review, Vol. 30, No. 4, pp. 157-179.

Sun S. and Pugh M., 2002. Properties of Thermo Chemically Processed Dual-Phase Steels Containing Fibrous Martensite, Materials Science \& Engineering, Vol. 335, pp. 298-308.

Swarr T. and Krauss G., 1976. The Effect of Structure on the Deformation of asQuenched and Tempered Martensite in an Fe-0.2 Pct C Alloy, Metallurgical Transactions, Vol. 7 (A), pp. 41-48.

Szewczyk A.F. and Gurland J., 1982. A Study of The Deformation and Fracture of a Dual-Phase Steel, Metallurgical Transactions, Vol. 13 (A), pp. 1821-1826.

Thomason P.F., 1990. Ductile Fracture of Metals, Pergamon Press, Oxford, UK.

Tipper C.F., 1962. Brittle Fracture Story, The University Press, Cambridge, UK.

Tomita Y., 1990. Effect of Morphology of Second-Phase Martensite on Tensile Properties of Fe-0.1C Dual Phase Steels, Journal of Materials Science, Vol. 25, pp. 51795184.

Tomota Y., Kuroki K., Mori T. and Tamura I., 1976. Tensile Deformation of Two-Phase Alloys: Flow Curves of $\alpha-\gamma$ Fe-Cr-Ni Alloys, Materials Science and Engineering, Vol. 24, pp. 85-94. 
Tomota Y., 1987. Effects of Morphology and Strength of Martensite on Cyclic Deformation Behavior in Dual-Phase Steels, Materials Science and Technology, Vol. 3, pp. 415-421.

Torquato S., 2002. Random Heterogeneous Materials - Microstructure and Macroscopic Properties, Springer, New York.

Tvergaard V., 1981. Influence of Voids on Shear Band Instabilities Under Plane Strain Conditions, International Journal of Fracture Mechanics, Vol. 17, pp. 389-407.

Tvergaard V., 1996. Effect of Void Size Difference on Growth and Cavitation Instabilities, Journal of the Mechanics and Physics of Solids, Vol. 44, pp. 1237-1253.

Tvergaard V., 1998. Interaction of Very Small Voids with Large Voids, International Journal of Solids and Structures, Vol. 35 (30), pp. 3989-4000.

Tzika P.A., Boyce M.C. and Parks D.M., 2000. Micromechanics of Deformation in Particle Toughened Polyamides, Journal of the Mechanics and Physics of Solids, Vol. 48, pp. 1893-1929.

Underwood E.E., 1970. Quantitative Stereology, Addison-Wesely Publishing Company, Inc., Philippines.

Weck E. and Liestner E., 1983. Metallographic Instructions for Colour Etching by Immersion-Part II: Behara Colour Etchants and their Different Variants, Deutscher Verlag Fur Schweisstechnik, cited by Metals Handbook, $9^{\text {th }}$ Edition, Vol. 9, Metallography and Microstructures, ASM, Metals Park, Ohio, 1985.

Weng G.J., 1990. The Overall Elastoplastic Stress-Strain Relations of Dual-Phase Metals, The Journal of the Mechanics and Physics of Solids, Vol. 38 (3), pp. 419-441.

Whitney J.M. and McCullough R.L., 1990. Micromechanical Material Modeling, Vol. 2, Technomic Publishing Company, Inc., Lanchester, Pennsylvania, USA.

Williams D.B. and Carter C.B., 1996. Transmission Electron Microscopy, Plenum Press, New York, USA.

Wilsdorf H.G.F., 1983. Review Paper: The Ductile Fracture of Metals: A Microstructural Viewpoint, Materials Science Engineering, Vol. 59, pp. 1-39.

Winchell P.G. and Cohen M., 1962. The Strength of Martensite, Transactions of ASM, Vol. 55, pp. 347-361.

Zhang Z., 2001. Nonlinear Fracture and Damage Mechanics, A Complete Gurson Model, Edited by Aliabadi M. H., WIP Press, Southampton, Boston, UK, pp. 223-248. 